volume 6

ISSUE 5

2018 sett./ott.

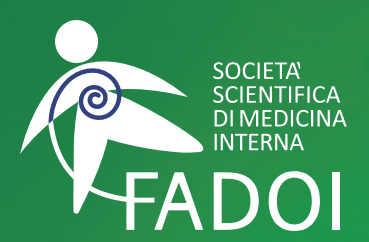

FEDERAZIONE

DELLE ASSOCIAZIONI

DEI DIRIGENTI

OSPEDALIERI

INTERNISTI

Editor in Chief

Paola Gnerre

Supervisor Editor

Roberto Nardi

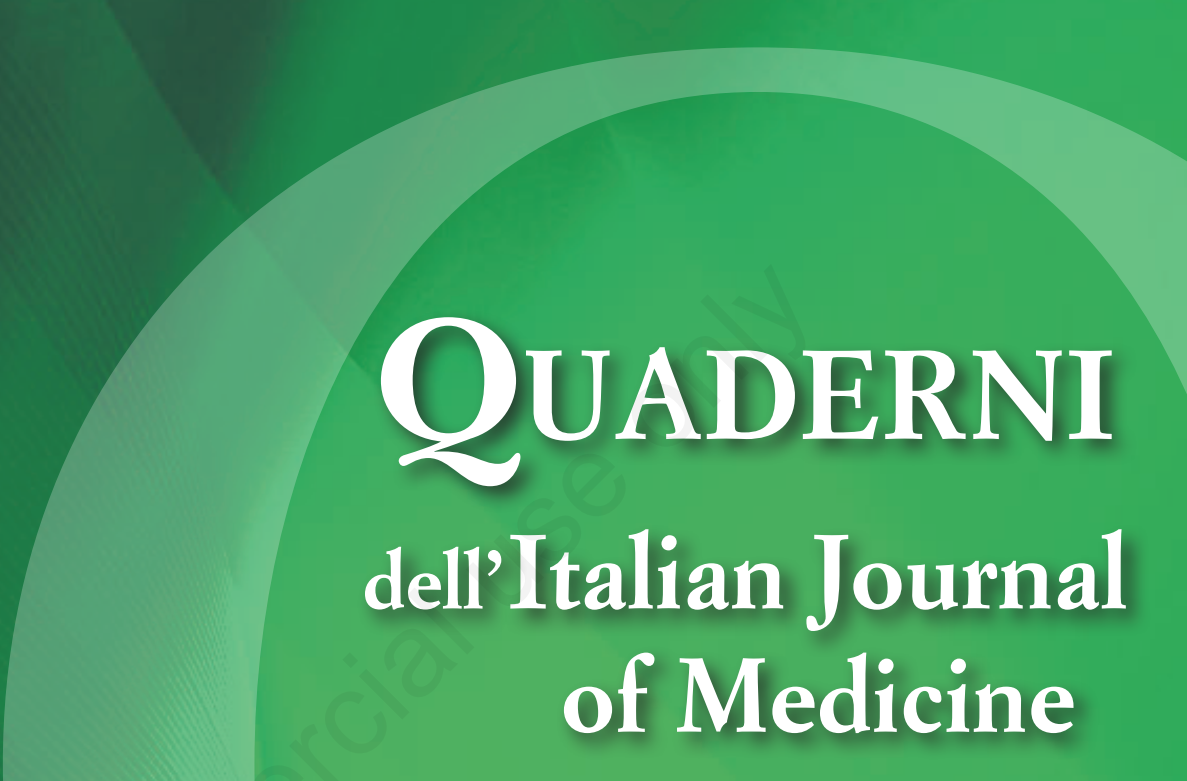

A Journal of Hospital

and Internal Medicine

The official journal of the Federation of Associations of Hospital Doctors on Internal Medicine (FADOI)

\title{
Nursing clinical competence in area medica
}

Guest Editors: F. Bertoncini, C. Gatta, G. Pentella 


\section{PRESIDENTE ELETTO}

Dario Manfellotto, Roma, Italy

\section{PAST PRESIDENT}

Mauro Campanini, Novara, Italy

\section{SEGRETARIO}

Micaela La Regina, La Spezia, Italy

\section{SEGRETARIO VICARIO}

Andrea Montagnani, Grosseto, Italy

\section{STAFF DI SEGRETERIA}

\begin{tabular}{ll}
\hline Comunicazione & Paola Gnerre, Savona, Italy \\
Ricerca & Roberta Re, Novara, Italy \\
Formazione & Maurizia Gambacorta, Todi $(P G)$, Italy
\end{tabular}

\section{TESORIERE}

David Terracina, Roma, Italy

\section{STAFF DI TESORERIA}

Francesco D'Amore, Roma, Italy

\section{PRESIDENTE FONDAZIONE FADOI}

Mauro Campanini, Novara, Italy

\section{COORDINATORE}

Giuseppe Augello, Canicatti ( $A G)$, Italy

DIPARTIMENTO PER LA RICERCA CLINICA "CENTRO STUDI FADOI"

Direttore $\quad$ Francesco Dentali, Varese, Italy

Supervisor per la Ricerca Giancarlo Agnelli, Perugia, Italy

DIPARTIMENTO PER LA FORMAZIONE

E AGGIORNAMENTO

Direttore Mauro Silingardi, Bologna, Italy

COORDINAMENTO FORMAZIONE

AREA CENTRO-NORD

Francesco Orlandini, La Spezia, Italy

COORDINAMENTO FORMAZIONE

AREA CENTRO-SUD

Generoso Uomo, Napoli, Italy

\section{COORDINATORE COMMISSIONE GIOVANI}

Flavio Tangianu, Oristano, Italy

MEMBRO FISM E RESPONSABILE

PER L'INNOVAZIONE IN MEDICINA INTERNA

Antonino Mazzone, Legnano (MI), Italy

\section{ITALIAN JOURNAL OF MEDICINE}

Editor in Chief Giorgio Vescovo, Padova, Italy

RESPONSABILE DEI

QUADERNI DELL'ITALIAN JOURNAL OF MEDICINE

Paola Gnerre, Savona, Italy

SUPERVISOR EDITOR DEI

QUADERNI DELL'ITALIAN JOURNAL OF MEDICINE

Roberto Nardi, Bologna, Italy

DELEGATO FADOI ITALIAN STROKE ORGANIZATION E CONSULTA CARDIOVASCOLARE

Michele Stornello, Siracusa, Italy

\section{RAPPORTI CON EFIM}

Antonio Luca Brucato, Bergamo, Italy

Gualberto Gussoni, Milano, Italy

Ombretta Para, Firenze, Italy

Giorgio Vescovo, Padova, Italy

\section{RESPONSABILE SITO NAZIONALE}

E COMUNICAZIONE

Salvatore Lenti, Arezzo, Italy

RESPONSABILE SISTEMA GESTIONE QUALITÀ

Franco Berti, Roma, Italy

RESPONSABILE CLINICAL COMPETENCE

Antonino Mazzone, Legnano (MI), Italy

RESPONSABILE AREA ECOGRAFIA

Francesco Cipollini, Ascoli Piceno, Italy

Marcello Romano, Catania, Italy

\section{RESPONSABILE MEDICINA DI GENERE}

Cecilia Politi, Isernia, Italy

\section{RESPONSABILE AREA NUTRIZIONE}

Roberto Risicato, Siracusa, Italy

Luciano Tramontano, Praia a Mare (CS), Italy

RESPONSABILE AREA DI CLINICAL GOVERNANCE

Giovanni Iosa, Cesenatico (FC), Italy

Stefano De Carli, Udine, Italy 


\section{Italian Journal of Medicine}

A Journal of Hospital and Internal Medicine

\section{COORDINATORE}

Giuseppe Augello, Canicattì ( $A G)$, Italy

\section{DIPARTIMENTO PER LA RICERCA CLINICA} CENTRO STUDI FADOI

Direttore Francesco Dentali, Varese, Italy

Supervisor per la Ricerca Giancarlo Agnelli, Perugia, Italy

SEGRETERIA

Grazia Panigada, Pescia (PT), Italy

RESPONSABILE AREA MALATTIE CARDIOVASCOLARI

Paolo Verdecchia, Assisi (PG), Italy

STAFF AREA MALATTIE CARDIOVASCOLARI

Cecilia Becattini, Perugia, Italy

Pierpaolo Di Micco, Napoli, Italy

Fernando Gallucci, Napoli, Italy

Alessandro Squizzato, Varese, Italy

\section{RESPONSABILE AREA MALATTIE INFETTIVE}

Ercole Concia, Verona, Italy

\section{STAFF AREA MALATTIE INFETTIVE}

Anna Maria Azzini, Verona, Italy

Gianluca Giuri, Castelnovo ne'Monti (RE), Italy

Matteo Giorgi Pierfranceschi, Piacenza, Italy

Carlo Tascini, Pisa, Italy

\section{RESPONSABILE AREA MALATTIE RESPIRATORIE}

Leonardo Fabbri, Reggio Emilia, Italy

\section{STAFF AREA MALATTIE RESPIRATORIE}

Bianca Beghè, Reggio Emilia, Italy

Piera Boschetto, Ferrara, Italy

Gaetano Cabibbo, Modica (RG), Italy

Francesco Corradi, Firenze, Italy

Francesco Ventrella, Cerignola $(F G)$, Italy

RESPONSABILE AREA MALATTIE REUMATOLOGICHE

Carlo Salvarani, Reggio Emilia, Italy

STAFF AREA MALATTIE REUMATOLOGICHE

Laura Morbidoni, Senigallia (AN), Italy

Nicolò Pipitone, Reggio Emilia, Italy

Tito D'Errico, Napoli, Italy

RESPONSABILE AREA MALATTIE METABOLICHE

Roberto Vettor, Padova, Italy

\section{STAFF AREA MALATTIE METABOLICHE}

Tiziana Attardo, Agrigento, Italy

Giovanni Gulli, Savigliano (CN), Italy

Ada Maffettone, Napoli, Italy

Maurizio Nizzoli, Forli, Italy

RESPONSABILE AREA ORGANIZZATIVA

IN MEDICINA INTERNA

Antonio Greco, San Giovanni Rotondo (FG), Italy

\section{STAFF AREA ORGANIZZATIVA IN MEDICINA INTERNA}

Marco Candela, Jesi (AN), Italy

Giovanni Mathieu, Pinerolo (TO), Italy

Valentino Moretti, San Daniele del Friuli (UD), Italy

Filomena Pietrantonio, Brescia, Italy

Elisa Romano, La Spezia, Italy

DIRETTORE DIPARTIMENTO PER LA FORMAZIONE E AGGIORNAMENTO

Mauro Silingardi, Bologna, Italy

COORDINAMENTO DI AREA FORMATIVA CENTRO-NORD

Francesco Orlandini, La Spezia, Italy

\section{COORDINAMENTO DI AREA FORMATIVA CENTRO-SUD}

Generoso Uomo, Napoli, Italy

STAFF DIPARTIMENTO PER LA FORMAZIONE

E AGGIORNAMENTO

Roberto Frediani, Chieri (TO), Italy

Marco Grandi, Sassuolo (MO), Italy

SEGRETERIA DIPARTIMENTO PER LA FORMAZIONE E AGGIORNAMENTO

Luigi Magnani, Voghera (PV), Italy

\section{BOARD SCIENTIFICO}

Clelia Canale, Reggio Calabria, Italy

Fabrizio Colombo, Milano, Italy

Giuseppe De Matthaeis, Città Sant'Angelo (PE), Italy

Massimo Giusti, Torino, Italy

Luca Masotti, Cecina (LI), Italy

Nicola Mumoli, Livorno, Italy

Maurizio Ongari, Porretta Terme (BO), Italy

Ruggero Pastorelli, Colleferro (RM), Italy

Fulvio Pomero, Savigliano (CN), Italy

Roberto Risicato, Siracusa, Italy

Antonio Sacchetta, Treviso, Italy

Giancarlo Tintori, Pisa, Italy

\section{COMMISSIONE TECNICHE E METODICHE INNOVATIVE} DI FORMAZIONE E VERIFICA

\section{Responsabile dell'Innovazione}

in Medicina Interna Antonino Mazzone, Legnano (MI), Italy

\section{STAFF}

Francesco Dentali, Varese, Italy

Andrea Montagnani, Grosseto, Italy

Filippo Pieralli, Firenze, Italy

\section{DIRETTORE SCIENTIFICO FONDAZIONE FADOI}

Gualberto Gussoni, Milano, Italy

\section{REFERENTI SLOW MEDICINE}

Roberto Frediani, Chieri (TO), Italy

Luigi Lusiani, Castelfranco Veneto (TV), Italy

RESPONSABILI AREA DOLORE

Domenico Panuccio, Bologna, Italy

Giuseppe Civardi, Piacenza, Italy

\section{AREA CRITICA IN MEDICINA INTERNA}

Carlo Nozzoli, Firenze, Italy 


\title{
QUADERNI - Italian Journal of Medicine
}

\author{
NURSING CLINICAL COMPETENCE IN AREA MEDICA \\ Guest Editors: F. Bertoncini, C. Gatta, G. Pentella
}

RASSEGNE

Introduzione

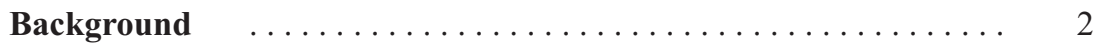

La nursing clinical competence. ................... 2

Il concetto di clinical competence in medicina interna ......... 4

Declinare e classificare i livelli di competenza ............ 4

Aree di assistenza nei contesti di area medica ............. 5

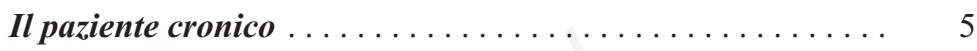

Scompenso cardiaco....................... 6

La broncopneumopatia cronica e ostruttiva . . . . . . . . 8

La cirrosi epatica . . . . . . . . . . . . . . . . . 11

La pancreatite . . . . . . . . . . . . . . . . . . . 12

Il diabete mellito . . . . . . . . . . . . . . . . . . . . . . 13

Il delirium . . . . . . . . . . . . . . . . . . . . . . . 13

La depressione ............................ 14

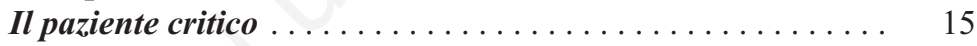

L'ipertensione........................... 17

La trombosi venosa profonda . . . . . . . . . . . . . . . 18

L'ischemia cerebrale . . . . . . . . . . . . . . . . . 20

La disfagia ............................ 25

Gli squilibri idroelettrolitici.................... 28

Le polmoniti . . . . . . . . . . . . . . . . . . . . . . 29

I sanguinamenti gastro-intestinali . . . . . . . . . . . . . 30

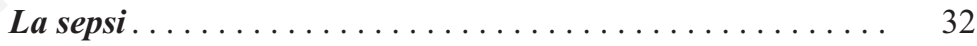

Il monitoraggio cardiaco .................... 32

La ventilazione non-invasiva . . . . . . . . . . . . . . . 34

L'ecografia operativa bedside .................... 35

Somministrazione di terapia e chemioterapia. . . . . . . . . . 37

Le lesioni da pressione. . . . . . . . . . . . . . . . . . . . 41

Il dolore .............................. 42

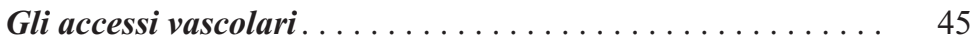

Il paziente fragile ........................ 46

La dimissione difficile . . . . . . . . . . . . . . . . 48

Il fine vita . . . . . . . . . . . . . . . . . . . . $\quad 49$

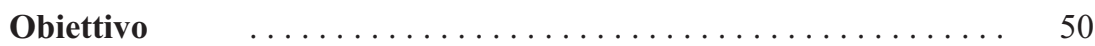

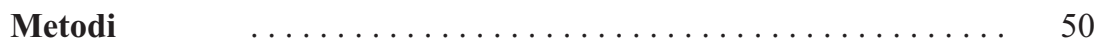

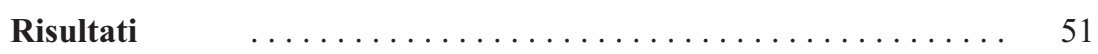

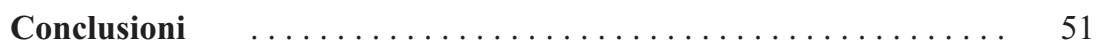

Bibliografia $\quad \ldots \ldots \ldots \ldots \ldots \ldots \ldots \ldots \ldots \ldots \ldots \ldots \ldots \ldots \ldots \ldots \ldots, 71$ 


\title{
Nursing clinical competence in area medica
}

\author{
Fabio Bertoncini, ${ }^{1}$ Claudia Gatta, ${ }^{2}$ Giovanna Pentella ${ }^{3}$ \\ ${ }^{1}$ Gruppo ricerca Associazione A.N.İ.M.O., ASL Biella; ${ }^{2}$ Referente formazione e Past-President Associazione A.N.Ì.M.O., \\ ASL Biella; ${ }^{3}$ Presidente Associazione A.N.İ.M.O., Ospedale Buon Consiglio Fatebenefratelli, Napoli, Italia
}

\section{Introduzione}

Negli ultimi anni, la Federazione delle Associazioni dei Dirigenti Ospedalieri Internisti (FADOI) ha pubblicato sull'Italian Journal of Medicine un interessante ed innovativo lavoro riguardante le Clinical Competence che devono essere acquisite dai medici internisti per l'esercizio della professione all'interno dei contesti di Medicina Interna*. La suddetta pubblicazione dibatte il concetto di Clinical competence come risultato delle conoscenze tecniche, abilità, qualità professionali, manageriali, relazionali operative di ogni singolo specialista nel suo contesto di cura. Risulta inoltre che le caratteristiche distintive del professionista in medicina interna siano basate su elementi quali: pluripotenza (capacità di sviluppo e integrazione di conoscenze e competenze multiple), (capacità di adattamento e modifica delle priorità d'intervento sia per il paziente singolo, che risposta alle necessità epidemiologiche della zona), interdipendenza funzionale (con la consapevolezza che la varietà di tipologia di paziente richiede scambio reciproco tra le diverse figure professionali, ma allo stesso tempo di qualcuno che sia referente del caso specifico) misure economiche di risparmio (che richiedono una gestione efficiente dei posti letti). Tutte queste caratteristiche mettono in luce la necessità di avere professionisti in formazione continua, consapevoli del proprio ruolo nel contesto organizzativo, con capacità di valutazione multidimensionale.

Sempre rimanendo all'interno del contesto di area medica, un'altra professionalità che interviene nei processi di cura dei cittadini che afferiscono a questi contesti è quella infermieristica; all'interno della

Corrispondente: Fabio Bertoncini, Gruppo ricerca Associazione A.N.İ.M.O., ASL Biella, Italia.

E-mail: fabio.bertoncini@hotmail.it

Articolo pubblicato secondo la Creative Commons Attribution NonCommercial 4.0 License (CC BY-NC 4.0).

CCopyright F. Bertoncini et al., 2018

Licensee PAGEPress, Italy

QUADERNI - Italian Journal of Medicine 2018; 6(5):1-83 professione infermieristica è tanto attuale quanto necessario far chiarezza e sistematizzare quell'insieme di informazioni presenti in letteratura, molte volte influenzate dalle culture professionali dei Paesi di riferimento, circa le competenze che l'infermiere deve possedere, sviluppare e consolidare all'interno della Medicina Interna attuale.

Ecco la nascita di questo lavoro che, partendo dai Project Work elaborati all'interno della prima esperienza italiana di Master Universitario di primo livello in Assistenza Infermieristica in Area Medica: Nursing della complessità, cura e assistenza nell'instabilità cronica dell'Università degli Studi del Piemonte Orientale viene proposto come primo tentativo di dar luce ad un corpo di competenze sistematizzate all'interno di ogni singolo processo di assistenza.

I processi verranno trattati partendo dalla sfera delle cronicità, seguiranno trattando le fasi acute, si evolveranno all'interno degli ambiti di natura tecnica per concludersi con i percorsi difficili.

Un ringraziamento per i contributi forniti va a Laura Biasio per l'assistenza al paziente cronico, Caterina Vitiello per l'assistenza al paziente con scompenso cardiaco, Roberta Gallo per l'assistenza al paziente con broncopneumopatia cronica e ostruttiva, Laura Grugnetti per l'assistenza al paziente con cirrosi epatica, Abaz Kojanaj per l'assistenza al paziente con pancreatite acuta, Nicola Leonetti per l'assistenza al paziente con Diabete Mellito, Gloria Aggio per l'assistenza al paziente con delirium, Matteo Pasquali per l'assistenza al paziente con depressione, Elena Simona Baetasu e Cristina Garza per l'assistenza al paziente critico, Dorin Barba per l'assistenza al paziente con ipertensione arteriosa, Arianna Bellotti per l'assistenza al paziente con trombosi venosa profonda, Dario Almasio per l'assistenza al paziente con ischemia cerebrale, Claudio Talarico per l'assistenza al paziente con disfagia, Stefania Botta per l'assistenza al paziente con squilibri idroelettrolitici, Barbara Curcio per l'assistenza al paziente con polmonite, Sonia Tallia per l'assistenza al paziente con sanguinamento gastro-intestinale, Valentina Tortone per l'assistenza al paziente con sepsi, Ilaria Nannini per l'assistenza al paziente con moni-

$\overline{\text { *Nardi R, et }}$ al. Ital J Med 2011;5S(1):3-13. 
toraggio cardiaco, Laura Aste per l'assistenza al paziente in ventilazione non invasiva, Paola Furlanetto per l'uso dell'ecografia operativa infermieristica, Juana Lisbel Magallanes Ronquillo e Benedetta Maria Coghi per la somministrazione di terapia e chemioterapia, Elisa Bortoloni per il contributo relativo alle lesioni da pressione, Eleonora Buora per l'assistenza al paziente con dolore, Irene Caccamo per il contributo relativo agli accessi vascolari, Lorenzo Savino per l'assistenza al paziente fragile, Simone Lazzati per l'assistenza durante il processo di dimissione difficile, Anna d'Angelo per l'assistenza al paziente in fine vita, Valentino Bissacca per il contributo circa la sistematizzazione dei dati relativamente alle aree di riferimento dell'instabilità.

\section{Background}

\section{La nursing clinical competence}

La competenza, dal latino cum-petere, esprime la piena capacità di orientarsi in determinati campi; la competenza professionale può essere descritta mediante il riferimento ad un curriculum di studio, ad un ambito di esperienza, ad un insieme codificato di abilità e conoscenze di un settore di attività con caratteristiche dettate dal contesto d'azione o di esercizio; in questa definizione di competenza, intesa in termini di conoscenze (Knowledge), capacità/abilità (Skill) e comportamenti (Attitude) è definito il valore aggiunto del professionista, che viene messa in atto in un determinato contesto per raggiungere un determinato scopo.(1)

Per ricostruire il dibattito sul tema delle competenze in Italia è necessario effettuare un breve excursus dei principali atti legislativi che regolamentano l'acquisizione delle competenze della professione infermieristica.(2)

L'abilitazione all'esercizio professionale delle professioni sanitarie è regolamentato dalla L. 26 febbraio 1999 n. 42 Disposizioni in materia di professioni sanitarie che definisce il campo di attività e responsabilità che si fonda su tre criteri guida rappresentati dal profilo professionale (D.M. 739/1994), dal codice deontologico e dalla formazione ricevuta. I criteri limite sulle competenze professionali previste sono la competenza della professione medica e delle altre professioni sanitarie.

La L. 10 agosto 2001 n. 251 Disciplina delle professioni sanitarie infermieristiche, tecniche, della riabilitazione, della prevenzione nonché della professione ostetrica, attribuisce alle Regioni, la valorizzazione e la responsabilizzazione delle funzioni e del ruolo delle professioni infermieristico-ostetriche al fine di contribuire alla realizzazione del diritto alla salute, al processo di aziendalizzazione del Servizio Sanitario Nazionale, all'integrazione dell'organizzazione del lavoro della sanità in Italia con quelle degli altri Stati dell'Unione.

Nella L. 1 febbraio 2006 n. 43 Disposizioni in materia di professioni sanitarie infermieristiche, ostetrica, riabilitative, tecnico-sanitarie e della preven- zione e delega al Governo per l'istituzione dei relativi ordini professionali viene riconosciuta la figura dell'infermiere specialista. Nell'art. 6 suddivide i professionisti sanitari in quattro tipologie di figure: $\mathrm{i}$ professionisti laureati o titolo equipollente (42/99); i professionisti coordinatori in possesso di master; gli specialisti in possesso di master; i dirigenti con laurea specialistica (magistrale 270/2004).

Le competenze avanzate nel campo infermieristico, negli ultimi anni, sono state oggetto di dibattito all'interno della professione e la mancanza di una definizione chiara e condivisa ha portato ad incomprensioni.

Il cambiamento della professione infermieristica è dettato, da un lato, dall'evoluzione dei bisogni dei cittadini e dall'altro, dall'evoluzione e dalle nuove richieste organizzative del Servizio Sanitario Nazionale (SSN). I bisogni dei cittadini, con problemi di salute sempre più caratterizzati da cronicità e disabilità, durante il lungo periodo di vita delle persone, richiedono competenze sempre più complesse e diversificate, di natura anche diversa rispetto al passato.

Le competenze avanzate degli infermieri rappresentano il nomarle sviluppo di una professione che, nei fatti, ha già compiuto in moltissime realtà sul territorio nazionale con ciò che la Federazione Nazionale dei Collegi Infermieri ha indicato nella bozza dell'accordo Stato-Regioni del 2013.

La Federazione dei Collegi Ipasvi, già Ordine delle Professioni Infermieristiche, ha presentato in Consiglio nazionale il nuovo modello di evoluzione delle competenze infermieristiche, che si richiama al Patto per la salute ed alla bozza di accordo Stato-Regioni sulle competenze avanzate. Competenze cliniche perfezionate, esperte e specialistiche. Due assi su cui disegnare la professionalità: quello della clinica, che rappresenta la linea del governo dei processi assistenziali, e quello della gestione, che rappresenta il governo dei processi organizzativi e delle risorse. I nuovi modelli formativi corrispondono ad un approfondimento delle competenze cliniche e gestionali, realizzato grazie ad una formazione adeguata, diretta a garantire l'irrobustimento ed approfondimento specialistico delle conoscenze e delle capacità assistenziali dell'infermiere in un determinato settore gestionale o in un'area clinica, in relazione ai bisogni di assistenza infermieristica, all'erogazione e valutazione di prestazioni e risultati ed al governo dei processi assistenziali specifici. Dal punto di vista della gestione è prevista un'espansione delle competenze attraverso un processo che parte da quelle disciplinari e va verso conoscenze e capacità proprie del governo delle risorse e dei processi organizzativi.

La bozza individua sei distinte aree di intervento: area cure primarie-servizi territoriali, area intensiva e dell'emergenza-urgenza, area medica, area chirurgica, area neonatologica-pediatrica ed area della salute mentale e delle dipendenze. Rispetto alla formazione post-base previste per l'infermiere dal profilo professionale (DM 739/1994) viene inserita l'area della chirurgia ed il superamento dell'area geriatrica a favore 
di un'area più generale, quella medica. L'art. 3 delinea le modalità ed i percorsi per lo sviluppo delle competenze professionali che si basano su formazione, ricerca ed esperienza professionale ed avranno come base normativa i contenuti dei profili professionali e gli ordinamenti didattici universitari. Il comma 2 dell'art. 3 definisce, all'interno del processo di accreditamento professionale, $i$ criteri per lo sviluppo delle competenze degli infermieri e la conseguente revisione dei modelli organizzativi.(3)

Le competenze avanzate vanno intese come sviluppo delle competenze con una diversa e più ampia modalità della presa in carico del paziente e dei processi assistenziali che permetta risposte più incisive alle mutate esigenze del contesto sanitario.

A livello centrale viene istituito l'osservatorio nazionale delle buone pratiche professionali e organizzative, come previsto dall'art. 5 della bozza.

In un successivo accordo con i soggetti sindacali si è istituita la cabina di regia, strumento per il confronto permanente unitario e partecipato sugli ambiti di sviluppo professionale, organizzativo e formativo. La cabina di regia è composta dai rappresentanti istituzionali e da un rappresentante per ogni organizzazione sindacale rappresentativa del comparto delle aree dirigenziali e dell'area convenzionata, deve intendersi come uno strumento per il confronto.(3)

Il Decreto Legislativo 28/01/2016 n. 15 di recepimento della direttiva 2013/55/Ue relativa al riconoscimento delle qualifiche professionali. Definisce le competenze che la normativa Ue riconosce agli infermieri: individuare autonomamente le cure infermieristiche, lavorare efficacemente con altri operatori del settore sanitario, orientare individui, famiglie e gruppi verso stili di vita sani e autoterapia, avviare autonomamente misure immediate per il mantenimento in vita, fornire autonomamente consigli, indicazioni e supporto alle persone bisognose di cure, garantire la qualità delle cure e di valutarle, comunicare in modo esaustivo e professionale e di cooperare con gli esponenti di altre professioni, analizzare la qualità dell'assistenza in un'ottica di miglioramento.

La competenza clinica viene intesa come la capacità di valutazione clinica del paziente, anche attraverso l'interpretazione di parametri clinici e di esami di laboratorio (clinical assessment) e di conoscenza di tecniche e presidi di utilizzo non abituali. L'infermiere esperto diviene il riferimento per l'equipe in grado di motivare le sue scelte e di interfacciarsi e dialogare con le altre professionalità che intervengono nel processo di cura del paziente.(4)

Il concetto di competenza infermieristica avanzata (advanced nursing practice, ANP) ha origine negli Stati Uniti ed in Canada negli anni ' 60 ed ha raggiunto il Regno Unito negli anni '80 come risposta ai cambiamenti dei bisogni di cura sanitaria. L'International Council of Nursing ha raccolto le definizioni di ANP dei vari Paesi ed ha elaborato una definizione: l'infermiere abilitato all'esercizio della professione che ha ac- quisito conoscenze esperte di base, capacità decisionali complesse e competenze cliniche per lo sviluppo della pratica, le cui caratteristiche dipendono dal contesto elo dal Paese nel quale esercita. Raccomanda il possesso di un titolo universitario post-base, master o dottorato in ambito clinico. I criteri per il riconoscimento del ruolo dell'infermiere con competenze avanzate più condivisi dalla comunità professionale internazionale sono la formazione post-base e la specifica esperienza nella pratica infermieristica riconducibile a cinque aree di competenza (pratica clinica, ricerca, istruzione/educazione, leadership e consulenza).(4)

La proposta formativa universitaria a livello italiano è molto eterogenea, si va dal master in area critica, sala operatoria, sanità pubblica, salute mentale che richiamano le specializzazioni di area proposte dal profilo dell'infermiere a master dove si acquisiscono competenze molto puntuali (esempio gestione accessi vascolari, ecografia). La numerosità dell'offerta formativa rappresenta per l'infermiere un'importante opportunità per lo sviluppo delle competenze specialistiche/avanzate.(5)

La figura infermieristica con competenza avanzata più diffusa è il clinical nurse specialist (CNS) le cui aree di specializzazione sono definite in popolazione (pediatrica, geriatrica), in setting (area critica, sanità pubblica), in patologia (diabete, oncologia), in tipologia di cure (prevenzione, riabilitazione, palliazione), in tipologia di problema (ferite, dolore, stomie). Gli studi hanno dimostrato che il contributo del CNS può influenzare gli esiti dei pazienti, ridurre i costi e la durata della degenza, gli accessi al Pronto Soccorso, la gestione del dolore, la soddisfazione per le cure infermieristiche e le complicanze mediche nei pazienti ospedalizzati.(6,7)

Secondo l'American Nursing Association (ANA) la pratica infermieristica avanzata si realizza attraverso l'esperienza clinica e con percorsi formativi di secondo livello (master o dottorati), che consentono di acquisire abilità e conoscenze specialistiche superiori e di estendere le loro competenze cliniche, attraverso lo sviluppo di capacità di ragionamento e di gestione dei problemi di assistenza ad elevata complessità. $(6,7)$

La competenza avanzata potrebbe essere intesa come sviluppo di competenze per migliorare la presa in carico della persona, la continuità assistenziale tra ospedale e territorio, il governo dei bisogni assistenziali, sanitari e socio sanitari delle persone, delle famiglie e della comunità assistita.(8)

Negli Stati Uniti ed in Inghilterra l'assistenza infermieristica avanzata si riferisce generalmente a cinque aree principali di competenza: clinica, educativa, di ricerca, di consulenza e di leadership $(9,10)$ l'infermiere con formazione superiore assume funzioni di supervisione, sostegno e consulenza ai colleghi facendosi promotore di processi di cambiamento. $(9,10)$

Il dibattito ha lo scopo di promuovere il riconoscimento di competenze cliniche degli infermieri esperti. La letteratura internazionale ha dimostrato le ricadute positive per gli esiti dei pazienti.(11) 
A livello internazionale la pratica infermieristica avanzata è riconosciuta come risposta alle mutevoli esigenze sanitarie.

Numerose evidenze documentano le ricadute positive della presenza di infermieri con competenze cliniche avanzate all'interno dei servizi. Modelli organizzativi centrati sul paziente, la presa in carico globale e la personalizzazione dell'assistenza infermieristica, miglior gestione pazienti critici o complessi, riduzione della riammissione. $(3,9,12,13)$

\section{Il concetto di clinical competence in medicina interna}

Il contesto organizzativo ha un ruolo importante per la definizione delle competenze. La competenza è intrinseca al modello organizzativo che richiede qualità, quantità e abilità trasversali. Il contesto è il luogo della reinterpretazione, della traduzione operativa e dell'adattamento delle abilità di carattere trasversale alle situazioni specifiche per ottenere risultati per l'organizzazione.

La direttiva del Dipartimento della Funzione Pubblica del 13/12/2001 sostiene che tutte le organizzazioni, per gestire il cambiamento e garantire un'elevata qualità dei servizi, devono fondarsi sulla conoscenza e sulle competenze. Devono pertanto assicurare la formazione permanente.

Le competenze richieste dal contesto lavorativo si suddividono in: conoscenze (generali, specifiche e di contesto), abilità (professionali specifiche e trasversali), caratteristiche personali e risorse psicosociali (motivazioni, autostima e qualità personali).

L'insieme delle attività prevalenti calate in un determinato contesto organizzativo derivano dall'evoluzione dei bisogni (cambiamento demografico, aumento della popolazione fragile, aumento persone malate che determinano una diversa domanda di salute) e dall'evoluzione del SSN in un sistema aziendalizzato, con un nuovo assetto organizzativo che prevede la responsabilizzazione dei professionisti e dei dirigenti con nuove competenze e diverso utilizzo delle competenze. Il modello di assistenza sanitaria centrato sulla persona, dove un tempo lo scopo era $\mathrm{Cu}$ rare ad oggi diviene sempre più prendersi cura.

La medicina interna rappresenta un' organizzazione ad elevata complessità, cui afferiscono pazienti tra loro assai differenti per tipologia e con flussi non programmabili. La complessità dei casi trattati risulta rilevante sia per gravità clinica, fragilità, comorbilità e disabilità sia per quadri atipici di presentazione. L'expertise dei clinici è rilevante quanto la medicina basata sulle evidenze e devono essere complementari tra loro.(14)

Da una revisione della letteratura emerge che, all'interno di una serie di competenze di valutazione, comunicazione, abilità di cura e abilità gestionali, sia l'infermiere novizio che l'infermiere esperto ritengono importante avere buone abilità cliniche. Per entrambi è essenziale essere in grado di integrare le conoscenze teoriche con le abilità pratiche. Infine per quel che riguarda le competenze gestionali risulta di fondamentale importanza il pensiero critico.(15)

Lo sviluppo di ragionamento critico nell'infermieristica implica la capacità di agire con flessibilità, identificando gli interventi per la risoluzione dei problemi nella specificità delle situazioni. Gli infermieri devono essere pensatori critici con lo scopo di esprimere un giudizio clinico.(16)

Alla luce di quanto detto si può affermare che una persona è in possesso di una competenza quando dimostra di avere le capacità, l'abilità e le conoscenze che gli permettono di svolgere un lavoro sapendo prendere decisioni e attivare risorse per la risoluzione dei problemi.(16)

\section{Declinare e classificare i livelli di competenza}

In letteratura sono disponibili diversi metodi di identificazione dei livelli di competenza posseduti. Uno di questi è rappresentato dalla scala di DreyfusDreyfus che si fonda sull'expertise e prevede cinque livelli di competenza:

1. Principiante novizio: il soggetto tende a seguire regole e principi comunicati dall'esterno senza tenere conto in modo esplicito del contesto in cui opera; si presenta in genere privo di flessibilità $\mathrm{e}$ di esperienza.

2. Principiante avanzato: il soggetto riesce a collegare le conoscenze con l'esperienza che sta progressivamente sviluppando e a selezionare i comportamenti da adottare.

3. Competente: le prestazioni sono basate su principi abbastanza generali derivati non solo dallo studio, ma soprattutto dall'esperienza e sanno adattarsi alle diverse circostanze. I soggetti sanno specificare, in modo adeguato rispetto ai casi particolari affrontati, gli obiettivi della loro azione e i mezzi per raggiungerli.

4. Competente avanzato abile: si ha una notevole capacità di inquadrare le situazioni da affrontare cogliendole nella loro complessità e riconoscendo analogie e differenze che esse hanno con situazioni simili affrontate in passato.

5. Esperto: il soggetto riesce a cogliere agevolmente un quadro completo e articolato delle situazioni da affrontare e ad agire per affrontarle in modo fluido, appropriato e senza sforzo.

Gli infermieri principianti sono focalizzati sul fare, possono assimilare poco di una situazione in quanto concentrati sul ricordarsi quando studiato. L'infermiere competente ha buona padronanza del proprio lavoro ed inizia a vedere le proprie azioni in funzione degli obiettivi da raggiungere. Il professionista abile ha la capacità di percepire la situazione come un insieme. L'infermiere esperto ha una profonda comprensione della situazione totale dovuta alla lunga esperienza.(16) 


\section{Aree di assistenza nei contesti di area medica}

\section{Il paziente cronico}

Le malattie croniche sono causa di morte per 40 milioni di persone ogni anno (circa il 70\% di tutti i decessi a livello globale). Il numero maggiore di decessi è dovuto alle malattie cardiovascolari (17,7 milioni di persone), seguite dai tumori ( 8,8 milioni), dalle malattie respiratorie ( 3,9 milioni) e dal diabete (1,6 milioni).(17)

In questo scenario, la lotta alle malattie croniche rappresenta una priorità di salute pubblica, sia nei Paesi più ricchi che in quelli più poveri. Da qui la necessità di investire nella prevenzione e nel controllo di queste malattie, da una parte riducendo i fattori di rischio a livello individuale, dall'altra agendo in maniera interdisciplinare e integrata per rimuoverne le cause.(18)

Se consideriamo inoltre il fatto che tali patologie assorbono più del $70 \%$ dei costi sanitari diretti, provocando altrettanti costi indiretti e sono in costante aumento a causa dell'invecchiamento generale della popolazione, possiamo affermare che le patologie croniche rappresentano l'emergenza del $3^{\circ}$ millennio.(19)

Uno degli obiettivi chiave prefissati dall'Organizzazione Mondiale della Sanità da raggiungere entro il 2020 è quello di aumentare la priorità assegnata alla prevenzione e al controllo di queste patologie, concordando obiettivi a livello regionale, nazionale ed internazionale, creare programmi di promozione della salute al fine di ridurre $\mathrm{i}$ fattori di rischio modificabili, individuare e formare operatori sanitari per facilitare il raggiungimento di questi obiettivi, fornire ad almeno il 50\% delle persone che ne possono beneficiare la terapia farmacologica o interventi di counselling per la prevenzione di eventi acuti cardio o cerebrovascolari.(20)

Uno degli aspetti sul trattamento delle patologie croniche nel quale emergono maggiori difficoltà riguarda proprio l'aderenza alla terapia.

La scarsa aderenza alle prescrizioni del medico è la principale causa di non efficacia delle terapie farmacologiche, associata a un aumento degli interventi di assistenza sanitaria, della morbilità, di ospedalizzazioni frequenti e della mortalità, rappresentando un danno sia per i pazienti che per il sistema sanitario e per la società. L'Italia è al secondo posto in Europa per indice di vecchiaia e dunque l'aderenza alle terapie risulta essere di prioritaria importanza per la sostenibilità del SSN.(21)

Già a partire dagli anni '70 Jean-Philippe Assal, endocrinologo svizzero, iniziò a porsi domande al fine di trovare una soluzione a queste importanti e più che mai attuali problematiche.

Assal capì fin da subito che le malattie croniche hanno caratteristiche peculiari che vanno tenute in debita considerazione, per evitare il fallimento della relazione terapeutica e quindi della cura. Egli sotto- linea la notevole differenza tra malattie acute e croniche: le prime presentano segni e sintomi bruschi ed evidenti, dove vi è urgenza di fare diagnosi precoce ed intervenire tempestivamente, al fine di individuare e mettere in atto il trattamento più opportuno e dove la compliance è generalmente buona. Le seconde spesso si sviluppano silenziosamente anche nell'arco di anni, causando seri danni visibili solo a distanza di tempo dall'esordio della malattia, possono causare uno shock emotivo legato all'incertezza del futuro e dei cambiamenti che si renderanno necessari sul piano personale, familiare, professionale ed economico. A differenza della persona affetta da una patologia acuta il paziente cronico prova un sentimento di perdita della salute e dell'integrità, si trova inoltre a confrontarsi con una situazione estremamente difficile e conflittuale: accettare di essere malato, farsi carico in prima persona della propria cura, che durerà per tutta la vita. Il paziente cronico è chiamato ad essere un soggetto attivo, un partner nelle cure poiché giorno dopo giorno dovrà essere in grado di curarsi adeguando la terapia alle condizioni che di volta in volta possono mutare. Per chi lo assiste non si tratta quindi solo di fare una diagnosi e prescrivere una terapia ma far sì che il paziente diventi capace di farsi carico della propria patologia, fornendogli sia le conoscenze $\mathrm{e}$ le abilità necessarie per poter realizzare una buona cura, sia un sostegno emotivo e motivazionale, senza il quale la cura non potrebbe essere seguita in maniera regolare e continuativa.(19)

Nella patologia cronica la vita del paziente viene modificata in modo irreversibile ed è in continua evoluzione, causandone una gestione costante e complessa. Queste variazioni nei modelli di malattia e i trattamenti con risultati incerti creano incertezza sulla prognosi, la chiave per una gestione efficace è quindi la comprensione di queste diverse tendenze e il loro ritmo: l'obiettivo non è infatti la cura ma il mantenimento di una vita piacevole ed indipendente. Nella maggior parte dei casi i medici non riescono a individuare con precisione queste tendenze, il paziente invece le conosce meglio e può fornire informazioni e preferenze complementari alle conoscenze professionali del curante.(22)

Assal propose quindi, per sostenere il paziente nella gestione della malattia cronica, di utilizzare il processo di educazione terapeutica.

L'educazione terapeutica è nata come nuovo paradigma di cura per le malattie croniche che si propone di migliorare non solo le conoscenze dei pazienti, ma soprattutto le competenze, il saper fare, e le capacità relazionali, saper essere; vuole inoltre favorire l'autonomia della scelta ed un senso di responsabilità del malato per renderlo protagonista attivo della cura.(23)

Gli operatori sanitari tendono a parlare ai pazienti delle loro malattie piuttosto che ad addestrarli nella gestione del quotidiano. Il trattamento delle patologie a lungo termine risulta essere soggettiva- 
mente meno soddisfacente di quanto potrebbe essere, poiché la qualità dell'assistenza dipende ancora molto dalla capacità del paziente di gestire la propria condizione, oltre che dai farmaci ed altre forme di tecnologia medica. Anche se gli operatori sanitari in generale risultano essere competenti nella diagnosi e nella scelta della terapia, solitamente non sono preparati né sull'educazione terapeutica del paziente né sui metodi di un'efficiente assistenza a lungo termine. Lo scopo principale è l'addestramento della persona assistita nelle abilità di auto-gestione ed il cercare di adattare il trattamento alla sua particolare situazione di cronicità; in questo modo, indirettamente si contribuisce a ridurre i costi dell'assistenza a lungo termine sia per i pazienti che per la società. L'obiettivo è di produrre un effetto terapeutico che vada ad assommarsi a quelli di altri interventi (farmacologici, di terapia fisica, ecc.). Si tratta quindi di un processo continuo, integrato nell'assistenza sanitaria, incentrato sul paziente, basato su una consapevolezza organizzata, sull'informazione, sull'apprendimento dell'auto-cura e sul supporto psicologico riguardo alla malattia, i trattamenti prescritti, l'assistenza, l'ospedale e gli altri ambiti assistenziali, i comportamenti legati alla salute e alla malattia. Esso tiene conto dei processi di adattamento del paziente (coping con la malattia, locus di controllo, convinzioni riguardo alla salute, percepito socioculturale) e dei suoi bisogni soggettivi ed oggettivi, sia espressi che non. E'un processo dinamico, che deve essere adattato al corso della malattia, al paziente ed al suo modo di vivere, deve essere strutturato, organizzato e fornito in maniera sistematica a tutte le persone assistite attraverso una varietà di mezzi, è multiprofessionale, intraprofessionale ed intersettoriale. Centrale è quindi la figura degli operatori sanitari che, se formati adeguatamente, possono contribuire a migliorare la qualità della vita dei pazienti, nonché a prolungarla, elevare la qualità dell'assistenza in generale e diminuire $\mathrm{i}$ costi medici, personali e sociali e di conseguenza la spesa totale.(24)

\section{Scompenso cardiaco}

L'insufficienza cardiaca (IC) o scompenso cardiaco (SC) è una sindrome clinica complessa definita come l'incapacità del cuore di fornire il sangue in quantità adeguata rispetto all'effettiva richiesta dell'organismo o la capacità di soddisfare tale richiesta solamente a pressioni di riempimento ventricolari superiori alla norma.(25)

Classicamente si distinguono:

- Scompenso sistolico, in cui è principalmente compromessa la contrattilità del miocardio;

- Scompenso diastolico, in cui si osserva un alterato rilasciamento del cuore durante la fase di riempimento ventricolare.

È frequentemente utilizzata anche la distinzione tra scompenso sinistro, in cui prevalgono i sintomi e segni clinici di congestione polmonare e ipoperfusione sistemica da deficit di funzione del ventricolo sinistro e scompenso destro, in cui si osserva prevalentemente un quadro di congestione venosa sistemica dovuta a disfunzione del ventricolo destro.

Nelle fasi più avanzate dell'insufficienza cardiaca, peraltro, tali distinzioni vengono meno in quanto la funzione cardiaca è compromessa in maniera globale.(26)

Anche un cuore sano può occasionalmente risultare scompensato, sia quando la richiesta funzionale a cui è sottoposto supera quella massima che esso è in grado di fornire, come può avvenire in alcune incontrollate prestazione agonistiche, sia quando la sensazione di stanchezza non è seguita da un adeguato riposo (scompenso relativo).

In condizioni patologiche, invece, l'insufficienza cardiaca può manifestarsi sia in caso di maggiore richiesta funzionale al cuore (insufficienza da sforzo), sia in assenza di tale fattore (insufficienza completa $o$ a riposo).

Inoltre, l'IC può essere acuta quando gli effetti si manifestano in maniera improvvisa, oppure cronica quando la gravità di tali effetti va incontro ad un progressivo incremento.(27)

Nei soggetti più giovani, lo $\mathrm{SC}$ è più frequente fra quelli di sesso maschile in quanto la cardiopatia ischemica, la causa scatenante più diffusa, si sviluppa nelle decadi di età meno avanzate. Fra gli anziani, invece, la prevalenza è simile in entrambi i sessi.(28)

La prevalenza globale dello $\mathrm{SC}$ è in aumento come conseguenza dell'invecchiamento della popolazione, dell'aumentata sopravvivenza dopo un evento coronarico e dell'efficacia delle misure preventive per i pazienti ad alto rischio o che sono sopravvissuti ad un primo evento.(29)

Lo SC costituisce il 5\% di tutte le cause di ospedalizzazione per un episodio acuto, è riscontrabile nel $10 \%$ dei pazienti ospedalizzati e rappresenta circa il $2 \%$ della spesa sanitaria.(30)

Per ridurre i sintomi e migliorare la prognosi occorre: (31)

- Restrizione nell'assunzione del sodio nella dieta, al fine di ridurre la ritenzione idro-salina, e diminuzione dell'assunzione di liquidi;

- Perdita di peso nei pazienti obesi

- Evitare la sedentarietà: un aumento dell'esercizo fisico, meglio se inserito nell'ambito di un programma riabilitativo permette di aumentare in modo significativo la capacità funzionale).(32)

Molto spesso i piani di dimissione ed i programmi di follow-up si rivelano carenti, non incentivando l'adozione dei comportamenti di autocura e sfociando in un supporto inadeguato ai pazienti e in un trattamento non ottimale. La scarsa o quasi nulla aderenza alla terapia e alle misure dietetiche ed il mancato riconoscimento dei sintomi 
si verificano frequentemente e possono essere la causa di oltre un terzo di tutte le ospedalizzazioni.

I programmi gestionali hanno lo scopo di migliorare l'outcome mediante la pianificazione di follow-up strutturati che comprendano gli interventi di educazione del paziente, l'ottimizzazione della terapia, il supporto psicosociale e la possibilità di facile accesso alla cura.
I programmi di gestione dello SC prevedono un approccio multidisciplinare che coordina la cura lungo il continuum della malattia attraverso la catena dell'assistenza prestata dai vari servizi del sistema sanitario. Un esempio di adeguato follow-up del paziente affetto da insufficienza cardiaca, con un metodo di allertamento precoce di scompenso, è rappresentato dalla Figura 1.

\section{EARLY WARNING SYSTEM IN HEART FAILURE}

\section{Un modello assistenziale utile} per la prevenzione dei ricoveri ripetuti per scompenso cardiaco

Nardi, R, Progetto OSCAR, 1998

\section{PERCORSO PRE-OSPEDALIERO DEL PAZIENTE CON SCOMPENSO CARDIACO 2}

II MMG effettua la valutazione clinica del paziente

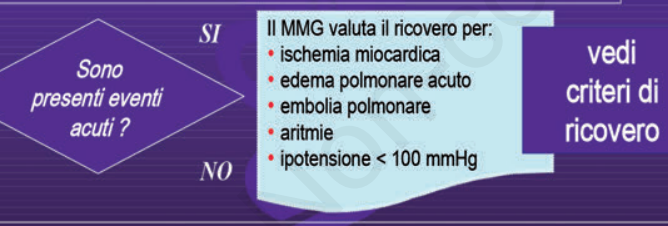

II MMG controlla l'introito di liquidi e ottimizza la terapia diuretica: furosemide per os $0 \uparrow$ posologico o sostituzione con torasemide 0 aggiunta antialdosteronici oppure: somministrazione di furosemide e.v. Prescrizione di controlli ematici urgenti: emocromo, $\mathrm{Na}-\mathrm{K}$, creatinina, azotemia. ECG urgente a domicilio

\section{TERAPIA DIURETICA}

\section{PERCORSO PRE-OSPEDALIERO DEL PAZIENTE} CON SCOMPENSO CARDIACO 1

Insorgono i primi segni/sintomi

di peggioramento dell'insufficienza cardiaca

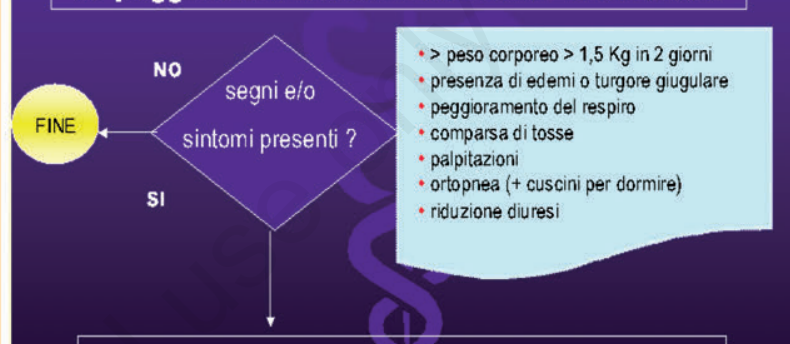

I'infermiere/ il paziente/ il famigliare/ informa il MMG

\section{TRIAGE PRECOCE}

\section{PERCORSO PRE-OSPEDALIERO DEL PAZIENTE} CON SCOMPENSO CARDIACO 3

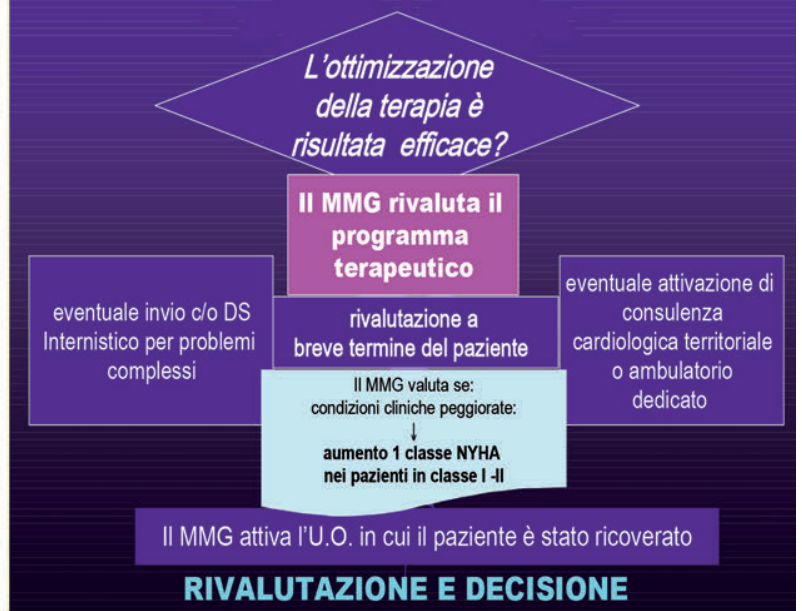

MMG, Medico di Medicina Generale; Na-K, Sodio-Potassio;

ECG, Elettrocardiogramma;

DS, Day-Service;

NYHA, New York Heart Association;

os, Somministrazione orale;

e.v., endovena.
Modificato da: Corazza G., Nardi R. Prime esperienze di governo clinico-assistenziale. Lo scompenso cardiaco: il progetto OSCAR (ottimizzazione della terapia e della gestione dello scompenso cardiaco nell'anziano con ricoveri ripetuti). Nursing Manage Inferm 2003;9:37-47.

Figura 1. Un possibile algoritmo per il riconoscimento precoce del peggioramento del paziente affetto da scompenso cardiaco. 
Un team multidisciplinare per lo SC è costituito da infermiere, cardiologo, medico internista e fisioterapista, nonché da altre figure e servizi specialistici dell'assistenza sanitaria.

In vista della dimissione è fondamentale l'attività di educazione sanitaria rivolta al paziente e/o al suo caregiver con particolare riferimento all'aderenza terapeutica, allo stile di vita e alla corretta alimentazione. Possibilmente, i pazienti devono imparare a riconoscere i sintomi predittivi di una possibile riacutizzazione della patologia e ad adottare misure di auto assistenza: devono essere in grado di rilevare autonomamente i parametri vitali, monitorare la diuresi nelle 24 ore, misurare la circonferenza addominale e il peso corporeo, gestire un adeguato stile di vita e un'equilibrata alimentazione, effettuare un controllo periodico dei valori ematici.(33)

Subito dopo la dimissione, viene raccomandato di sottoporre il paziente a visita ambulatoriale allo scopo di valutare le condizioni cliniche, di identificare gli obiettivi e di pianificare una strategia terapeutica efficace. È emerso che il follow-up domiciliare o ambulatoriale determina una riduzione significativa del numero delle ospedalizzazioni.

I pazienti che presentano caratteristiche cliniche di $\mathrm{SC}$ avanzato con sintomatologia refrattaria alla terapia dimostratasi ottimale sulla base delle evidenze disponibili, hanno una prognosi a breve termine infausta e devono essere ritenuti candidati ad un programma strutturato di cure palliative.

Gli interventi devono mirare ad un miglioramento della qualità di vita, al controllo dei sintomi, all'identificazione precoce e al trattamento degli episodi di aggravamento, nonché al perseguimento di un approccio olistico alla cura del paziente che comprenda il benessere fisico, psicologico, sociale e spirituale.

\section{La broncopneumopatia cronica e ostruttiva}

La broncopneumopatia cronica e ostruttiva (BPCO) è una malattia dell'apparato respiratorio caratterizzata da un'ostruzione irreversibile delle vie aeree, di entità variabile a seconda della gravità, cronica e causata da una risposta infiammatoria prolungata delle vie aeree ad un agente irritante (ad esempio fumo di sigaretta). La malattia (nota in inglese come COPD, chronic obstructive pulmonary disease) è solitamente progressiva ed è associata a uno stato di infiammazione cronica del tessuto polmonare. La conseguenza a lungo termine è un vero e proprio rimodellamento dei bronchi, che provoca una riduzione consistente della capacità respiratoria.

Non esiste al momento una cura efficace, ma sono disponibili diversi trattamenti per controllare i sintomi e per evitare pericolose complicanze. Fondamentale è invece la prevenzione, per ridurre al minimo il fattore di rischio principale: il fumo di sigaretta.(34)

Interessa entrambi i sessi ed è fumo-dipendente poiché riconosce appunto il fumo, attivo e passivo, quale maggiore fattore di rischio. Circa il 40-50\% dei fumatori sviluppa una BPCO. La sua prevalenza è maggiore nei Paesi industrializzati rispetto a quelli in via di sviluppo, aumenta con l'aumentare dell'età, ed è in crescita nel sesso femminile. Tali dati riflettono in gran parte l'aumentato uso di tabacco e l'incremento dell'età media delle popolazioni nei Paesi in via di sviluppo.(35)

La BPCO non è curabile, ma il trattamento può alleviare i sintomi, migliorare la qualità della vita $\mathrm{e}$ ridurre il rischio di morte. I sintomi della BPCO peggiorano progressivamente e persistono causando difficoltà respiratoria durante l'esercizio físico e conducendo all'affanno anche a riposo.(35)

La broncopneumopatia cronica ostruttiva è una patologia ampiamente prevenibile e trattabile (ma non guaribile), è al momento la quarta causa di morte nel mondo, ma le proiezioni la pongono al terzo posto tra le cause di morte entro il 2020.

Si prevede che il costo della BPCO aumenti nei prossimi decenni a causa della continua esposizione ai fattori di rischio e per l'invecchiamento della popolazione. $(36,37)$

La maggior parte dei dati nazionali evidenzia che la diagnosi di BPCO si riscontra in meno del $6 \%$ della popolazione adulta, dimostrando probabilmente che la BPCO è diffusamente sotto-stimata e sotto-diagnosticata. Anche la sotto-stima e la sotto-diagnosi di BPCO influenzano l'accuratezza dei dati sulla mortalità.(37)

Secondo i dati 2017, in Italia fuma il $28.6 \%$ dei maschi ed il 20,3\% delle femmine per un totale di $12.2 \mathrm{mi}-$ lioni di persone. Sulla base dei dati oggi disponibili è previsto a livello mondiale il notevole aumento della morbilità per $\mathrm{BPCO}$ con uno spostamento dal $12^{\circ} \mathrm{al} 6^{\circ}$ posto tra le malattie croniche.(37)

La BPCO è causa di circa il 50\% delle morti per malattie dell'apparato respiratorio che, a loro volta, rappresentano la terza causa di morte in Italia (dopo malattie cardiovascolari e neoplasie).(38)

E' dimostrato che sono realisticamente raggiungibili molti obiettivi, come il miglioramento dei sintomi, della tolleranza allo sforzo e dello stato generale di salute, la riduzione del numero e delle gravità delle riacutizzazioni e la prevenzione delle complicanze della BPCO.(39)

Per quanto riguarda gli interventi clinico-assistenziali sulle malattie croniche, si concorda sul ruolo primario che rivestono la prevenzione da un lato e l'educazione terapeutica dall'altro. Questo può avvenire attraverso il cambiamento delle abitudini e dello stile di vita dei singoli individui e della collettività, l'identificazione precoce delle persone a rischio e il rallentamento dell'evoluzione clinica, migliorando l'autocontrollo della malattia da parte della persona a rischio o affetta da BPCO.(38)

- Interventi non farmacologici per pazienti che hanno avuto una recidiva recente:

C'è forte interesse negli interventi non farmacologici per i pazienti che hanno recentemente sviluppato una riacutizzazione di BPCO. Questi interventi com- 
prendono l'autogestione specifica della malattia, la riabilitazione polmonare, il follow-up medico precoce, le visite a domicilio degli operatori sanitari e i programmi di assistenza integrata.(40)

\section{- Supporto nutrizionale:}

Un basso indice di massa corporea, e in particolare una ridotta massa magra, si associa a risultati clinici peggiori in pazienti con BPCO. In pazienti malnutriti affetti da BPCO, il supplemento nutrizionale aumenta il peso corporeo e porta ad un miglioramento della forza dei muscoli respiratori e, complessivamente, della qualità di vita legata alla salute.

I pazienti denutriti affetti da BPCO dovrebbero ricevere un supplemento dietetico. Questa raccomandazione è basata su risultati derivati da revisioni sistematiche, che indicano un effetto positivo del peso corporeo, massa grassa e massa magra, quando ai pazienti con BPCO viene offerto supplemento dietetico da solo (soprattutto se i pazienti sono malnutriti), oppure aggiunto all'esercizio fisico. La durata e la quantità ottimale del supporto dietetico non sono chiare. I pazienti che hanno ricevuto un'integrazione dietetica hanno avuto un miglioramento nel test del cammino dei sei minuti rispetto al basale, un miglioramento della forza muscolare ed un miglioramento dello stato di salute (solo nei pazienti malnutriti).(37)

- Panico, ansia e depressione:

Le cause dei sintomi di depressione e ansia nei pazienti con BPCO sono multifattoriali e comprendono fattori comportamentali, sociali e biologici. La riabilitazione respiratoria può aiutare a ridurre i sintomi di ansia. L'efficacia degli antidepressivi nei pazienti con BPCO è dubbia, probabilmente a causa degli aspetti metodologici degli studi pubblicati, campionamenti ridotti, eccessiva eterogeneità tra $i$ gruppi di confronto. La terapia comportamentale cognitiva e gli interventi mente-corpo (ad es. la terapia basata sulla consapevolezza, lo yoga e il rilassamento) possono ridurre ansia e depressione; in particolare, gli interventi mente-corpo possono anche migliorare indicatori fisici come la funzione respiratoria, la dispnea, la capacità fisica e la fatica in pazienti affetti da BPCO e con problemi psicologici.(37)

\section{- Fatica:}

La fatica nei pazienti con BPCO può giovarsi dell'educazione all'auto-gestione, della riabilitazione respiratoria, del supporto nutrizionale e degli interventi su mente-corpo.(37)

\section{- Cure palliative e fine vita:}

Lo scopo delle cure palliative è ridurre la sofferenza dei pazienti e delle loro famiglie, tramite la valutazione globale e il trattamento dei sintomi fisici, psichici e spirituali vissuti dal paziente. I pazienti con una patologia cronica e limitante come la BPCO dovrebbero essere informati che, qualora la situazione diventasse critica, loro stessi o i loro familiari potrebbero trovarsi nella condizione di dover decidere se un percorso di cure invasive e intensive sia in grado di raggiungere gli obiettivi di cura personali, e se hanno la volontà di sottoporsi a tali trattamenti. I medici dovrebbero sviluppare e migliorare metodi per aiutare i pazienti e le loro famiglie a prendere decisioni consapevoli che siano in linea con i valori dei pazienti stessi. Un approccio semplice e strutturato che faciliti queste comunicazioni può aiutare a migliorare la frequenza e la qualità della comunicazione, implementando il punto di vista del paziente.(37)

Gli argomenti considerati appropriati in un programma educativo includono: l'abolizione del fumo; le informazioni di base sulla BPCO; le informazioni generali sull'approccio terapeutico e su specifici aspetti della terapia farmacologica (farmaci e inalatori); le strategie per aiutare a ridurre la dispnea; i consigli sul quando cercare e chiedere aiuto; il prendere decisioni consapevoli durante le riacutizzazioni; la valutazione di cure palliative e percorsi di fine vita. L'intensità e il contenuto di questi messaggi educazionali dovranno variare a seconda della gravità della patologia del paziente.(37)

E'inoltre importante valutare le capacità residue della persona nelle attività di vita quotidiana, come la capacità di camminare nella stanza, la capacità di nutrirsi e dormire senza grave dispnea così da garantire il normale rientro al domicilio. E inoltre da valutare la piena comprensione della gestione terapeutica domiciliare da parte del paziente o di chi se ne prende cura, garantendo un coinvolgimento consapevole del paziente, della famiglia e del medico curante nella terapia domiciliare.

Durante il ricovero sarà da attuare l'addestramento all'impiego della terapia inalatoria e rimarcare l'importanza dell'aderenza alla terapia prescritta, l'importanza dell'attività fisica e i principi per una corretta alimentazione e controllo del peso.

È importante verificare la necessita di presidi e la fornitura di ossigenoterapia, se necessaria.

Verificare la compliance alla terapia farmacologia (dose, ora, modo di assunzione ed effetti collaterali) e alla dieta, informare il paziente di prestare attenzione all'eventuale comparsa di modificazione della sintomatologia o modificazioni delle abitudini di vita (ad esempio: non esce più di casa, ha ridotto la propria attività in casa, ecc.), variazioni del peso o di altri parametri vitali che possono suggerire un peggioramento della malattia.

La persona affetta da BPCO è l'elemento centrale di un sistema di gestione integrata ed ha la responsabilità di una gestione consapevole della propria malattia. L'educazione del paziente affetto da BPCO all'autocura (self-care), all'autocontrollo ed all'autonomia decisionale, è utile nel ridurre il rischio di ricovero per riacutizzazione, attenuare l'ansia e la depressione, migliorare la risposta alle riacutizzazioni.(41)

L'educazione del singolo paziente e la valutazione del rischio di riacutizzazioni, la valutazione delle necessità del paziente, delle sue preferenze e dei suoi obiettivi, dovrebbe formare un disegno personalizzato di educazione all'autogestione. 
La cessazione del fumo è considerato l'intervento più efficace ed economicamente più vantaggioso, nella maggior parte delle persone, per ridurre il rischio di sviluppare la BPCO e arrestarne la progressione.(37)

Le strategie per aiutare il paziente a smettere di fumare, dovrebbero essere applicate da ogni operatore sanitario, in quanto è dimostrato che anche minime raccomandazioni e indicazioni per la cessazione del fumo (il cosiddetto minimal advice) risultino utili alla riduzione dell'abitudine al fumo. Alcuni tipi di intervento sono risultati i più efficaci: il sostegno comportamentale e la terapia farmacologica.(39)

- Educazione terapeutica:

L'educazione sanitaria del paziente affetto da $\mathrm{BPCO}$ può migliorare la sua capacità di controllare la malattia con contenuti che devono comprendere:

- informazioni sulle caratteristiche della malattia (cronicità, progressione, future complicanze, ecc.);

- identificazione e controllo dei fattori di rischio e di aggravamento;

- conoscenza degli obiettivi realistici della terapia;

- conoscenza dell'utilizzo dei farmaci (specie quelli per via inalatoria) e della modalità di riconoscimento e trattamento tempestivo di una riacutizzazione;

- conoscenza delle modalità di monitoraggio dell'andamento della malattia;

- conoscenza del modo di utilizzo dell'ossigenoterapia e della terapia ventilatoria domiciliare, quando necessarie;

- conoscenza di come programmare la vita e le attività quotidiane, sulla base anche delle aspettative e delle preferenze del paziente.(39)

Nella pratica clinica la scelta dell'inalatore (per i prodotti che sono disponibili in diverse formulazioni) è condizionata da fattori legati al paziente:

- abilità del paziente nell'usare correttamente l'inalatore prescritto;

- preferenza espressa dal paziente;

- tempo necessario per istruire il paziente al corretto impiego dell'inalatore e per monitorarlo;

- possibilità di usare lo stesso tipo di inalatore per somministrare tutti i farmaci necessari al trattamento;

- comodità del paziente, della famiglia e dello staff medico per somministrare il farmaco;

- necessità di manutenzione, trasportabilità e pulizia dell'inalatore;

- costo.(42)

- Attività fisica:

Vi sono evidenze che l'attività fisica nei pazienti con BPCO sia ridotta. Questo determina una spirale negativa di inattività, che predispone i pazienti ad un aumentato rischio di ridotta qualità di vita e maggior tasso di ricoveri ospedalieri e mortalità.

La combinazione di esercizi di resistenza a carico costante e di allenamento della forza ad intervalli è risultata migliore dei due metodi considerati da soli. Quando possibile, si preferisce un allenamento fisico cardiovascolare di resistenza, generalmente in moda- lità aerobica, al 60-80\% del massimo sforzo fisico tollerato o della frequenza cardiaca, oppure fino al grado 4-8 di dispnea secondo la Scala Borg o lo score di fatica (moderato e grave).

Gli esercizi di allenamento degli arti superiori migliorano la forza delle braccia e la resistenza; questo garantisce un miglioramento della capacità funzionale per le attività degli arti superiori. Gli esercizi respiratori aumentano la forza dei muscoli inspiratori, ma questo non si traduce nè in una miglior performance nè in un calo della dispnea, a meno che non siano inclusi in un programma completo di riabilitazione polmonare.(37)

\section{- La riabilitazione respiratoria:}

La riabilitazione andrebbe considerata parte integrante del trattamento del paziente; di solito prevede l'intervento di vari operatori sanitari, per assicurare la copertura ottimale dei tanti aspetti considerati. I pazienti dovrebbero essere sottoposti a una attenta valutazione prima dell'arruolamento, che comprenda l'identificazione degli obiettivi del paziente, le specifiche necessità legate alla salute, la valutazione dell'abitudine al fumo, lo stato nutrizionale, la capacità di autogestione, la capacità di comprendere i problemi di salute, la misura dello stato psicologico, la valutazione del contesto sociale, delle comorbidità, così come pure delle abilità e delle limitazioni fisiche.

Andrebbero inseriti esercizi per gli arti superiori e inferiori e cammino libero; andrebbero anche incorporati esercizi di elasticità, allenamento dei muscoli inspiratori e stimolazione elettrica neuromuscolare. In tutti i casi, l'intervento riabilitativo (obiettivo, intensità) andrebbe personalizzato per ottenere il massimo della propria capacità funzionale residua. I benefici della riabilitazione respiratoria nei pazienti con $\mathrm{BPCO}$ sono notevoli, e la riabilitazione ha dimostrato di essere la più efficace strategia terapeutica per migliorare la dispnea, lo stato di salute e la tolleranza allo sforzo. Revisioni sistematiche recenti hanno mostrato che nei pazienti con riacutizzazione recente $(\leq 4$ settimane dal precedente ricovero) la riabilitazione respiratoria è in grado di ridurre in misura statisticamente significativa successive riospedalizzazioni e la mortalità. $(37,43)$

- Autogestione:

Il self-management (ovvero l'autogestione da parte del paziente) e il tutoraggio da parte dei professionisti dovrebbero essere componenti fondamentali del $\mathrm{Mo}$ dello di Cura della Cronicità, nel contesto del sistema di gestione del servizio sanitario. Lo scopo del selfmanagement è di motivare, coinvolgere e guidare i pazienti verso un adattamento positivo dei loro comportamenti e verso lo sviluppo delle capacità necessarie per migliorare la gestione della malattia.

I medici e gli infermieri devono spingersi oltre l'approccio puramente educativo o limitato a dare consigli, a favore di un approccio che aiuti i pazienti ad imparare ad adottare strategie e abilità sostenibili di autogestione. Oltre a correggere i fattori di rischio comportamentali (es. fumo, dieta, esercizio), il self- 
management dovrebbe coinvolgere i pazienti nell'identificare e trattare i segni e i sintomi della malattia, mantenere una buona aderenza terapeutica (inclusa la terapia farmacologica e comportamentale), mantenere contatti regolari con gli operatori sanitari e affrontare le conseguenze psicosociali della malattia. $(37,44)$

- Programma di cura integrata:

La BPCO è una malattia complessa che richiede l'azione di molte figure sanitarie, che devono collaborare strettamente.(41)

La cura integrata necessita di essere personalizzata sullo stadio di gravità della malattia e sulla comprensione dei problemi di salute di quel paziente.(37)

\section{La cirrosi epatica}

La cirrosi epatica è il risultato comune di danni cronici al parenchima del fegato causato da una varietà di malattie epatiche. Deriva dalla sostituzione del tessuto epatico con cicatrice fibrotica e dalla rigenerazione di tessuti e noduli, portando progressivamente a disfunzione epatica e complicanze cliniche come l'ipertensione portale, carcinoma epatocellulare (HCC), insufficienza epatica e la morte.

La cirrosi è una causa principale della mortalità e morbilità in tutto il mondo e rappresenta un peso importante sulle risorse sanitarie. L'epidemiologia della cirrosi epatica varia con la posizione geografica e le condizioni socioeconomiche. Le principali cause della cirrosi nei paesi occidentali sono epatite cronica $\mathrm{C}$ e malattia epatica da alcool. In Oriente, ci sono variazioni nell'epidemiologia della cirrosi epatica all'interno di diversi paesi asiatici. L'epatite cronica $\mathrm{C}$ è la causa prevalente della cirrosi epatica in Giappone, mentre l'epatite B cronica è la principale eziologia della cirrosi in Cina, Corea e parti del sud-est asiatico. In Nepal e Thailandia, la causa predominante è la cirrosi legata all'alcol.(45)

La cirrosi è la principale indicazione per 5500 trapianti di fegato ogni anno in Europa.(46)

E' difficile valutare la prevalenza della cirrosi e probabilmente è più alta di quella riportata, poiché gli stadi iniziali sono asintomatici, quindi non è diagnosticato il disturbo.(46)

In uno studio epidemiologico che prendeva in considerazione una casistica di 2185 pazienti italiani con cirrosi diagnosticata nel 2001, è stato evidenziato che il fattore eziologico più comune era l'infezione cronica da virus HCV $(69,9 \%$ di paziente $\mathrm{HCV}-\mathrm{Ab}$ positivi, HCV come unica causa di epatopatia nel $51,1 \%$ ), seguito da infezione cronica da $\operatorname{HBV}(13 \%)$ e poi dall'alcool (12,3\% di soggetti con antigeni virali negativi). Inoltre è risultato che nel sud Italia l'eziologia $\mathrm{HCV}$-correlata era più frequente rispetto al nord, dove invece prevaleva la cirrosi alcol-correlata.(47)

Nonostante il rischio di evoluzione verso una condizione severa, la cirrosi epatica non dovrebbe più essere considerata una malattia terminale e incurabile; attualmente è infatti sempre più accettato il concetto di processo dinamico e almeno parzialmente reversibile, previa eliminazione dei fattori causali.(48)

Si stima che circa un quarto dei pazienti dimessi dopo un ricovero per cirrosi epatica scompensata verranno riammessi entro 30 giorni. Queste riospedalizzazioni sono state associate a un aumento della morbilità e della mortalità, e possono essere dannose finanziariamente per il sistema sanitario e inoltre possono essere parzialmente prevenibili.(49)

E'quindi fondamentale durante il ricovero fornire ai pazienti e ai loro famigliari un'adeguata educazione sugli stili di vita da adottare, la nutrizione, la prevenzione e la gestione delle complicanze.

Fornire assistenza infermieristica sicura e promuovere la qualità della vita ai i pazienti con cirrosi epatica può rappresentare una sfida attuale per gli infermieri a causa dell'elevata complessità della malattia e dei regimi terapeutici richiesti. Ci sono molte complicanze associate alle malattie croniche del fegato, che aumentano il potenziale numero di ospedalizzazioni e decessi. Per questo motivo, gli infermieri devono impiegare tutto il loro repertorio di conoscenze per riconoscere le più frequenti diagnosi infermieristiche in pazienti con cirrosi epatica per identificare la migliore strategia di pianificazione in base alle priorità e le strategie per fornire una terapia di supporto, migliorare la gestione dei sintomi e la prevenzione delle complicanze associate.(50)

È inoltre chiaro che la malattia epatica avanzata è associata ad una qualità della vita drasticamente ridotta. Sappiamo che alcuni fattori clinici, la qualità di vita dei pazienti con cirrosi epatica, includono la severità della malattia, la presenza di ascite o encefalopatie e iponatraemia. È quindi improbabile che un singolo intervento infermieristico globale migliorerà la qualità di vita nella malattia epatica avanzata, ma concentrandosi sistematicamente sui singoli fattori, potrebbero essere possibili miglioramenti complessivi.(51)

- La malnutrizione è altamente prevalente in pazienti con cirrosi epatica ed è presente nel 40-90\% di questa popolazione. La rilevanza di questa complicanza è l'impatto che ha sulla prognosi e sulle altre complicanze della malattia. Sono stati individuati diversi approcci per migliorare lo stato nutrizionale quali diete ad alto contenuto di fibre, l'integrazione con micronutrienti quali vitamine e minerali e l'esercizio fisico.(52)

- Tutti i pazienti con cirrosi dovrebbero essere sottoposti a screening per varici esofagee con endoscopia superiore in modo che venga determinato il rischio di emorragia e che la terapia profilattica possa essere somministrata a quelli con varici che presentano un maggior rischio di sanguinamento. Il paziente deve conoscere $\mathrm{i}$ cibi che possono provocare sanguinamento (temperature elevate del pasto) e le eventuali terapie che influiscono sulla coagulazione.

- I pazienti con cirrosi devono essere sottoposti a sorveglianza con ultrasuoni ogni sei mesi. Ogni 
paziente deve essere educato circa l'importanza della compliance terapeutica.

- Il riconoscimento precoce e il trattamento di infezioni localizzate (ad esempio, cistite, cellulite) possono aiutare a prevenire la batteriemia e la peritonite batterica spontanea (PBS). I pazienti devono saper riconoscere i segni e sintomi di un'eventuale infezione (dolore, febbre, astenia, torpore).

- Nei pazienti con cirrosi devono essere evitati agenti nefrotossici in quanto possono precipitare in insufficienza renale.

- I pazienti con cirrosi ospedalizzati sono esposti ad infezioni durante l'ospedalizzazione. I fattori che sono stati associati a infezioni secondarie acquisite in ospedale nei pazienti con cirrosi includono l'uso di cateteri vescicali, ventilazione meccanica e il posizionamento di accessi vascolari. Molti di questi interventi a volte sono necessari (ad esempio il posizionamento di cateteri vescicali per misurare l'uscita di urina). Tuttavia, ponderando attentamente l'utilità di questi interventi si può diminuire il rischio di acquisire un'infezione durante l'ospedalizzazione.(50)

- I pazienti con cirrosi devono essere molto attenti a proteggere il fegato da qualsiasi fattore che possa danneggiarlo ulteriormente. I vaccini contro l'epatite $\mathrm{A}$ e $\mathrm{B}$, per coloro che non sono già immuni, possono aiutare a prevenire ulteriori danni al fegato. Siccome le infezioni possono essere particolarmente dannose per le persone con cirrosi, è inoltre importante eseguire anche i vaccini contro l'influenza (1 volta all'anno), la difterite e il tetano (1 volta ogni 10 anni), e la pertosse (1 volta durante l'età adulta).(53)

\section{La pancreatite}

La pancreatite acuta $(\mathrm{PA})$ è un processo infiammatorio acuto del pancreas: presenta patogenesi complessa ancora oggi parzialmente ignota, pertanto oggetto di continui studi clinici. Manifesta un'evoluzione spesso imprevedibile ed è gravata da complicanze, che influenzano il decorso clinico.

A causa di tali complessità diagnostiche e terapeutiche l'infiammazione pancreatica è associata ad aumento significativo dei costi correlabili a morbilità, mortalità ed ospedalizzazione.(54)

Da una disamina della letteratura clinica la PA si presenta come la principale causa di ospedalizzazione tra le patologie gastro enterologiche negli USA.(55)

I principali fattori di rischio che predispongono il paziente allo sviluppo della PA sono i calcoli biliari e gli abusi di alcol. Numerose ricerche e studi di recentissimo sviluppo hanno messo in luce la presenza di nuovi elementi scatenanti la patologia quali ad esempio, fattori di natura infettiva (infezioni sistemiche o locali).(55)

Nel trattamento del paziente affetto da una patologia di tale entità è necessario porre particolare attenzione alla diagnosi, alla diagnosi differenziale, alle compli- canze, ai fattori prognostici, al trattamento ed alla prevenzione di possibili ricadute e/o di un decorso ingravescente caratterizzato da una cronicizzazione della patologia.(55)

L'infermiere che opera nell'ambito della Medicina Interna necessita di due differenti competenze specifiche da sviluppare e spendere in due differenti ambiti: tecnico ed educativo.(56)

Nell'ambito tecnico l'infermiere dovrà essere in grado di:

- Alleviare il dolore del paziente attraverso somministrazione di analgesici su prescrizione medica e monitorando attentamente l'efficacia e l'andamento dello stesso; sospendere l'alimentazione orale preferendo la via parenterale, sempre in seguito ad accertamenti medici mirati; eseguire l'igiene della bocca per ridurre i fastidi dovuti a secchezza della mucosa orale, soprattutto nel caso di digiuno;

- Migliorare lo stato nutrizionale attraverso la misurazione e la registrazione del peso corporeo del paziente, tutte le uscite dei liquidi incluse le urine, vomito, diarrea, sudorazione e verificare il bilancio idroelettrolitico, anche in collaborazione con dietisti e dietologi;

- Mantenere l'integrità cutanea attraverso posizionamento di presidi per prevenire ulcerazioni da pressione, causate da deficit nutrizionali, allettamento forzato e probabile irrequietezza; se il paziente è stato sottoposto ad intervento chirurgico è necessario controllare la ferita chirurgica e l'eventuale inserimento di drenaggi, controllare la cute attorno alla ferita per eventuali infezioni.

Nell'ambito educativo l'infermiere dovrà essere in grado di:

- Sviluppare un programma di educazione alla salute, anche in collaborazione con altre figure specialistiche (es. psicologo o educatore), che si occupi della spiegazione delle cause di insorgenza della patologia e del corretto stile di vita da seguire (riduzione dei livelli alcolici, dieta bilanciata priva di alimenti ricchi di grassi) in seguito all'evento acuto. Tale intervento può essere effettuato sia a mezzo orale, attraverso indicazione degli operatori sanitari, sia in forma scritta attraverso pieghevoli e brochure di supporto. La partecipazione a gruppi di auto mutuo aiuto e/o di un trattamento psicoterapico può favorire l'accettazione della condizione clinica del paziente e migliorare la compliance alla terapia da seguire;

- Coinvolgere la rete familiare come strumento principe necessario per una buona aderenza a quanto raccomandato, dal momento in cui è necessario che il paziente venga sostenuto e supportato nei cambiamenti necessari da effettuare nella sua vita;

- Favorire la creazione di una rete domiciliare di assistenza continua per monitorare le condizioni cliniche del paziente e l'aggiustamento di potenziali condotte nocive alla salute dello stesso (follow-up). 


\section{Il diabete mellito}

Il diabete è una delle patologie croniche a più ampia diffusione nel mondo, in particolare nei Paesi industrializzati, e costituisce una delle più rilevanti e costose malattie sociali della nostra epoca, soprattutto per il suo carattere di cronicità, per la tendenza a determinare complicanze nel lungo periodo e per il progressivo spostamento dell'insorgenza verso età giovanili.

Le cause del manifestarsi di quella che viene definita come una vera e propria epidemia sono da ricercarsi in almeno quattro fattori fondamentali:

- Proliferare delle abitudini alimentari scorrette;

- Crescita del numero di persone obese o in sovrappeso.

- Affermarsi di stili di vita sempre più sedentari.

- Invecchiamento della popolazione.

Dati recentemente pubblicati dall'International Diabetes Federation (IDF) (57) sostengono che nel 2010 oltre 284 milioni di persone fossero affetti da diabete e le proiezioni dell'organizzazione non sono rassicuranti: si prevede infatti che nel 2030 i diabetici adulti (fascia d'età 20-79) saranno 438 milioni. La diffusione del diabete sta crescendo in ogni regione del mondo ed è previsto che il tasso di prevalenza globale aumenterà dal 6,4\% del $2010 \mathrm{al}$ 7,7\% nel 2030 . Attualmente in Italia vivono almeno tre milioni di persone con diabete, cui si aggiunge una quota di persone, stimabile in circa un milione, che pur avendo la malattia, non ne sono a conoscenza.

Le disuguaglianze sociali agiscono fortemente sul rischio diabete. La prevalenza della malattia è, infatti più elevata nelle classi sociali più basse e tale effetto è più evidente in tutte le classi di età.(57)

Un aspetto di prioritaria importanza nell'assistenza ai pazienti con diabete risulta essere l'educazione terapeutica anche se la letteratura ci suggerisce che il ricovero ospedaliero non è il momento ideale per attivare una terapia educativa. I pazienti sono sofferenti, stressati, focalizzati sul problema che ha portato al ricovero, in un ambiente che non favorisce l'apprendimento, gli operatori sanitari sono oberati dall'impegno quotidiano del reparto, i turni non favoriscono la continuità del rapporto operatore-paziente, per cui educare all'autogestione della malattia diabetica in ospedale diventa un compito difficile e impegnativo.

Tuttavia durante la degenza i diabetici di nuova diagnosi e quelli che hanno iniziato il trattamento insulinico e l'autocontrollo della glicemia devono essere addestrati in modo da garantire una gestione sicura al rientro al domicilio nel momento della dimissione.

Per i diabetici già noti, il momento del ricovero può diventare occasione per verificare i comportamenti nella somministrazione e gestione della terapia insulinica o ipoglicemizzante orale e l'autocontrollo glicemico, individuando le eventuali criticità nella gestione della malattia da parte della persona assistita. Il momento del ricovero che deve essere inoltre, un'opportunità per rinforzare messaggi sulla corretta ali- mentazione e sullo stile di vita o per la verifica della presenza di eventuali lipodistrofie. È necessaria inoltre una collaborazione multidisciplinare tra l'equipe di reparto e il team diabetologico, che deve essere coinvolto fin dall'inizio attraverso la richiesta di consulenza.

Durante il periodo di ospedalizzazione dovranno essere intrapresi i seguenti interventi:

- Addestrare all'utilizzo della penna per insulina e la corretta tecnica iniettiva;

- Addestrare ad eseguire la rilevazione della glicemia;

- Addestrare alla compilazione del diario delle glicemie;

- Addestrare a riconoscere e a trattare l'ipoglicemia;

- Comunicare e concordare i valori range personalizzati per quel paziente;

- Verificare l'apprendimento e le tecniche.

Questi interventi rientrano in un set minimo di informazioni per garantire alla persona con diabete ospedalizzata di rientrare a domicilio in sicurezza. Ma fondamentale sarà il completamento del percorso educativo strutturato con il team di diabetologia, che può avvenire durante il ricovero attraverso la consulenza specialistica oppure programmando un incontro con il team del post ricovero per la presa in carico e i regolari follow-up (58).

\section{Il delirium}

Il delirium nell'adulto, o stato confusionale acuto, è una sindrome clinica caratterizzata da disturbi della coscienza, delle funzioni cognitive o della percezione, con comparsa acuta e ad andamento fluttuante. E' una condizione severa associata ad esiti negativi per il paziente la quale può essere prevenuta e i sintomi trattati se riconosciuti e affrontati con urgenza.

Gli anziani, le persone affette da deficit cognitivi, demenza, malattie gravi o fratture d'anca, sono le persone maggiormente a rischio di andare incontro a delirium. Circa il 20-30\% dei pazienti ricoverati nei reparti medici manifestano delirium; tra il $10 \%$ e il $50 \%$ delle persone sottoposte ad intervento chirurgico sviluppano delirium, con variabilità rispetto ai differenti interventi chirurgici (maggiore incidenza per interventi di fratture di femore operate in urgenza con età superiore ai 60 anni, dove la probabilità si stima intorno al 40-55\%).(59)

Nei reparti di cura con tempi di degenza più lunga, la prevalenza di delirium è inferiore al $20 \%$.

La prevalenza del delirium tende ad aumentare con l'aumentare dell'età.(60)

Nei pazienti medici la prevalenza è del $18 \%$ e, nei pazienti di età superiore ai 65 anni nelle prime 72 ore dall' ammissione in ospedale è del 6-54\% durante il ricovero.(59)

Il delirium è scarsamente riconosciuto e poco registrato nella documentazione clinica ma il suo riconoscimento e tempestivo trattamento può impedire il manifestarsi di importanti complicanze come: 
- aumentato rischio cadute e lesioni da decubito (cinque volte maggiore);

- prolungamento della degenza (da 9 a 21 giorni);

- evoluzione in demenza;

- trasferimento in strutture protette (maggiore del $47 \%)$;

- morte (rischio dieci volte maggiore 35-40\%).(59)

I fattori di rischio, che possono essere individuati attraverso l'uso di scale validate ad uso infermieristico, si possono dividere in predisponenti e precipitanti e sono legati all'età avanzata ( $>65$ anni), l'alterazione delle funzioni cognitive e/o demenza, patologia acuta in atto o intervento chirurgico; la presenza di questi fattori di rischio ci fa già identificare questo come un paziente a rischio.

Esistono poi fattori di rischio precipitanti legati al paziente come: esacerbazione di una condizione medica cronica, anestesia/intervento chirurgico, introduzione di nuovi farmaci anti psicotici, ictus cerebrale, dolore, ritenzione acuta di urine, stipsi, alterazione elettroliti, disidratazione e sepsi; fattori precipitanti legati all'ambiente come le modifiche e illuminazione ambientali, sovraccarico sensoriale; fattori precipitanti legati alle procedure come il tipo di anestesia, fratture femore, posizionamento catetere vescicale, cessazione del fumo, restrizione fisiche.

Se più fattori predisponenti sono identificati in un soggetto, risulta più alta la sua vulnerabilità e, per il manifestarsi della sindrome, sono necessari minori fattori precipitanti. La prevenzione quindi si fonda sull'identificare e agire sui fattori modificabili sia predisponenti che precipitanti.

Si possono distinguere tre sottotipi di delirium in relazione allo stato psicomotorio: una forma ipoattiva, una iperattiva e una mista. La forma ipoattiva è caratterizzata da letargia, apatia e stato confusionale ed è quello meno riconosciuto nei pazienti anziani in quanto scambiato con demenza o depressione.

Il delirium in forma iperattiva è caratterizzato da agitazione, confusione, aggressività, sintomi psicotici, allucinazioni e labilità emotiva. La forma mista unisce le caratteristiche dell'ipoattività e dell'iperattività con andamento fluttuante.(59)

I pazienti con delirium hanno scarsa capacità di mantenere l'attenzione con disturbi della memoria, soprattutto a breve termine, disturbi del linguaggio ed inversione del ritmo sonno-veglia.(59)

Se vengono quindi rilevati indicatori di delirium devono essere ricercati i sintomi indicati e deve essere condotta una valutazione attraverso scale validate come, ad esempio, la scala CAM (confusion assessment scale). Qualora sia difficile la distinzione tra delirium, demenza o delirium che aggrava una demenza, il delirium va trattato per primo.(61)

Punto cardine è avere consapevolezza che il paziente ricoverato in ospedale può essere a rischio di delirium. Questo per le importanti conseguenze di aumentato rischio di morte e/o demenza, può determinare un allungamento della degenza.(60)
L'approccio al paziente con delirium consiste nella correzione rapida, per quanto possibile, di tutti i fattori potenzialmente modificabili: mantenere la stabilità cardiovascolare, temperatura corporea, gestione del dolore, ossigenazione, bilancio idrico, il profilo glucidico e l'apporto calorico.(59)

Il trattamento della sintomatologia del delirium consiste di una parte non farmacologica e di una farmacologica. La prima mira al recupero dell'orientamento del paziente mantenendo il contatto visivo e tattile, il miglioramento delle capacità funzionali, della mobilità e l'uso di istruzioni verbali chiare e semplici; correzione di difetti acustici e visivi preesistenti. Inoltre ridurre al minimo i rumori e mantenere un'adeguata illuminazione, evitare cambi ambientali e mezzi di contenzione. Prevenire, inoltre cadute, lesioni da pressione, infezioni e declino funzionale.(59)

Fondamentale il coinvolgimento della famiglia e caregivers per identificare e riconoscere precocemente cambiamenti nel comportamento del paziente e, successivamente per la sua gestione per rassicurarlo e garantirne la sicurezza.(62)

La terapia farmacologica dovrebbe essere riservata nei casi in cui il paziente si presentasse agitato, delirante, aggressivo e dove le misure non farmacologiche non fossero in grado di controllare il quadro clinico o impedisse il trattamento della patologia acuta in atto. Si raccomanda l'uso di farmaci solo per breve tempo (short-term treatment) pari ad una settimana o meno. I farmaci più utilizzati in acuto sono aloperidolo, risperidone, olanzapina, quietapina. $(59,62)$

Inoltre una revisione sistematica di Neufeld et al. dell'aprile 2016 mostra la scarsa efficacia dell'uso di antipsicotici nella prevenzione ed il trattamento del delirium ed evidenzia la necessità di ulteriori studi in questo ambito. $(63,64)$

\section{La depressione}

Il disturbo depressivo, noto anche come disturbo depressivo maggiore (MDD), disturbo depressivo persistente (distimia), depressione endogena $o$ depressione clinica è una patologia psichiatrica caratterizzata da uno stato di umore depresso, ridotta autostima, perdita di piacere e/o interesse verso attività precedentemente piacevoli, comportamento irritabile oltre a cambiamenti cognitivi e somatici che intaccano le capacità e le funzioni dell'individuo.(65)

La depressione e nella sua espressione più estrema il suicidio, sono fenomeni complessi che provocano sofferenze intense nella vita delle persone colpite, delle loro famiglie, degli amici e della comunità.

Questi due fenomeni coesistono e si influenzano l'un l'altro, per questo motivo sono considerati entrambi importanti problemi di salute pubblica.(66)

Le stime dell'Organizzazione Mondiale della Sanità (OMS) riferiscono che la depressione rappresenta il 4,3\% delle patologie globali.(66)

Viene definita come una problematica planetaria 
poiché circa 300 milioni di persone appartenenti a tutte le fasce di età ne sono affette. Questa patologia ha visto un incremento dei casi del $18 \%$ dal 2005 al 2015.(67)

Essa è la principale causa di disabilità nei paesi industrializzati del mondo, in particolare per le donne.(66) Secondo il DSM V la prevalenza del MDD nell'età adulta è del 10-25\% nelle donne e del 5-12\% negli uomini, mentre quella del disturbo distimico raggiunge il $6 \%$ in ambedue i sessi.

La probabilità di avere un episodio depressivo maggiore entro i 70 anni è del $27 \%$ negli uomini e del $45 \%$ nelle donne: cifre che dimostrano in modo chiaro l'ampia diffusione di questa patologia, con una maggior diffusione tra il sesso femminile rispetto al maschile.(65)

Gli infermieri che lavorano con persone affette da MDD devono sempre considerare le capacità di coping associate alla depressione, devono ridurre gli atteggiamenti negativi, incrementare le capacità relazionali, far sviluppare all'individuo un'alleanza con il personale clinico, l'auto-miglioramento e potenziare il concetto di speranza della persona.

Questo approccio potrebbe essere decisivo nella rigenerazione delle funzioni professionali e psicosociali della persona, in qualità di miglioramento della vita, $\mathrm{e}$ nella prevenzione delle ricadute e delle recidive.

Gli Infermieri svolgono un ruolo importante nel prevenire le ricadute di disturbi depressivi e nel fornire la cura del paziente utilizzando approcci diversi. In particolare, l'infermiere specializzato può utilizzare la psicoterapia (più frequentemente il CBT) e i modelli di cura.

Sviluppato da Gordon nel 1982, il concetto di Modelli Funzionali della Salute è un modello assistenziale che standardizza la raccolta dei dati nella pratica infermieristica, semplifica il processo diagnosi e considera l'individuo con un approccio olistico.(68)

Nell'individuo affetto da MDD i principali modelli di salute alterati e le relative diagnosi infermieristiche NANDA (North American Nursing Diagnosis International), i cui obiettivi, per essere perseguiti, necessitano quasi sempre di collaborazione multidisciplinare, sono:

- Percezione-gestione della salute=Alterazioni nel mantenimento dello stato di salute; Inefficace gestione del regime terapeutico.

- Nutrizionale-metabolico=Alterazioni della nutrizione: Alto rischio di assunzione di quantità superiori al fabbisogno dell'organismo/Alterazioni della nutrizione: Assunzione di quantità inferiori al fabbisogno dell'organismo.

- Eliminazione=Stipsi.

- Attività ed esercizio fisico=Sindrome da deficit della cura di sè; Carenza attività ricreative; Fatigue; Intolleranza all'attività.

- Riposo e sonno=Disturbi del sonno (insonnia/ ipersonnia).
- Cognitivo/percettivo=Alterazione dei processi cognitivi; Conflitto decisionale.

- Percezione e concetto di sé=Ansia; Disperazione; Alterazione dell'autostima, Bassa stima di sé cronica; Alto rischio di autolesionismo.

- Ruolo e relazioni=Alto rischio di violenza contro se stessi o gli altri; Alterazione dei processi famigliari; Isolamento sociale; Alterazione della comunicazione verbale; Difficoltà di interazione sociale.

- Sessualità e riproduzione=Disfunzione sessuale.

- Coping/Tolleranza allo stress=Strategie di coping individuali inefficaci.

- Valori e convinzioni=Afflizione spirituale.

La depressione negli anziani nell'assistenza di base è una sfida complessa. Importante è approcciarsi alla persona con una valutazione fisica, psicosociale e spirituale. L'infermiere e l'infermiere specializzato devono sempre considerare la storia della persona ed essere sempre in allerta sui segni ed i sintomi al fine di riconoscere tempestivamente la problematica e migliorare la prognosi e gli outcomes funzionali.(69)

Sebbene l'educazione al paziente faccia parte della buona assistenza clinica, nella gestione della depressione e del suo trattamento essa ricopre un ruolo di particolare importanza, poiché questa condizione è spesso soggetta a miti, malainformazione, preconcetti e stigmatizzazione.

La maggior parte dei pazienti e delle loro famiglie hanno la necessità di comprendere cosa sia la depressione, da cosa è causata e come viene trattata, in questo modo viene fortificata l'alleanza e i pazienti tendono a seguire maggiormente le raccomandazioni date loro, come seguire il trattamento, il piano psicoterapico, monitorare segni e sintomi e comunicare i propri progressi con il clinico.

Gli infermieri sono incoraggiati ad utilizzare brochure informative con il paziente in modo da trasmettere le informazioni in modo chiaro, semplice e diretto circa le manifestazioni cliniche della depressione, la biologia sottostante, i fattori di rischio noti e le diverse opzioni di trattamento.(70)

\section{Il paziente critico}

Nell'ultimo decennio tutti i servizi ed i sistemi sanitari del mondo nei paesi ad economie sviluppate sono alle prese con le stesse problematiche: cambiamento demografico, crescita delle aspettative di salute dei cittadini e delle comunità, nuove tecnologie sanitarie a disposizione. $(71,72)$ Le malattie croniche rappresentano il principale problema di salute pubblica nei Paesi occidentali, $(71,73,74)$ oltre a minare a fondo la sostenibilità dei sistemi sanitari. Queste patologie sono responsabili del 92\% di tutte le morti nel nostro Paese, con una maggiore rilevanza delle patologie cardiovascolari (41\%) e dei tumori (28\%).(71) Il miglioramento delle cure e il continuo sviluppo delle tecnologie sanitarie (ad es. farmaci, vaccini, presidi, programmi di as- 
sistenza, percorsi diagnostico terapeutico assistenziali, organizzazione dei servizi) combinato al crescente invecchiamento della popolazione, pone notevoli pressioni gestionali ed economiche sui Sistemi Sanitari Nazionali.(71,75) L'accresciuta richiesta di salute si è riversata su tutti i settori della Sanità ma ha trovato sfogo negli Ospedali. Per le caratteristiche di questi pazienti (anziani e molto anziani, pluripatologici, complessi, affetti da malattie croniche, ma anche critici e non di rado con problematiche sociali), la maggior parte del carico assistenziale è stato assorbito dalla Medicina Interna, che è andata incontro a numerose trasformazioni e in parte si è reinventata.(76) Dalla sua posizione centrale nella nuova concezione dell'Ospedale per Intensità di Cure, la Medicina Interna ha dovuto dispiegare un variegato ventaglio di competenze che vanno dall'assistenza al paziente critico (ventilazione non invasiva, complicanze settiche, gestione di cateteri venosi centrali, procedure invasive, ecografia bedside, ecc.) al reinserimento sul territorio del paziente vulnerabile, dai percorsi assistenziali complessi in regime di day service alla continuità ospedale-territorio.(77) In questo tipo di organizzazione gli infermieri hanno l'opportunità di valorizzare le loro competenze, qualificare il loro ruolo nel processo assistenziale e contribuire alla costruzione di team multi-professionali che agiscano in sinergia per migliorare gli esiti dei pazienti.(78)

Il team medico-infermieristico dedicato all'assistenza necessita di competenze clinico assistenziali in precedenza prerogativa dei reparti intensivistici. Sono richieste competenze che riguardano il monitoraggio, la valutazione e l'interpretazione ed interventi immediati per le situazioni di criticità. L'utilizzo routinario di tecniche NIV, ecografiche e di amine presuppone formazione e addestramento specifici. $(74,78,79)$

In linea generale un paziente si definisce critico quando risulta portatore di una condizione attuale o recente di insufficienza acuta di un organo e/o di un sistema, o di più organi o sistemi, con conseguente rischio possibile per la vita, che richiede osservazione continua e prestazioni personalizzate. Il paziente diviene critico instabile quando presenta una alterazione della coscienza, con compromissione delle funzioni vitali (A: vie aeree, B: respiro, C: circolo), con pressione arteriosa sistolica $<90 \mathrm{mmHg}$ nonostante riempimento.(75) Queste alterazioni necessitano di una correzione in tempi più o meno brevi per evitare un peggioramento clinico che potrebbe degenerare fino all'arresto cardiorespiratorio. Buona parte degli arresti cardiocircolatori sono quasi sempre preceduti da un peggioramento delle condizioni cliniche del paziente, spesso misconosciute o sottostimate. Prevenire l'arresto cardiaco è fondamentale, dal momento che solo il $20 \%$ dei pazienti che hanno subito un arresto cardiocircolatorio riescono a sopravvivere. In generale, i segni clinici che si manifestano nelle condizioni critiche sono simili indipendentemente dal processo patologico sottostante, poiché essi riflettono una insufficienza funzionale dell'apparato respiratorio o cardiovascolare o del sistema nervoso. Le alterazioni dei segni vitali sono comuni, tuttavia i parametri fisiologici più significativi dei pazienti affetti da patologie acute vengono rilevati e riportati in cartella meno frequentemente di quanto sarebbe raccomandabile.(80)

Quindi il paziente critico può essere definito in base a due indicatori: la stabilitàlinstabilità e la complessità assistenziale infermieristica.

La stabilità si riferisce alla capacità di una persona di mantenere un equilibrio di stato stazionario, che permetta il normale espletamento delle funzioni fisiologiche.(75) È legata alla normalità o meno di funzioni vitali (ABC: vie respiratorie, respirazione, circolazione), pressione arteriosa, temperatura corporea, frequenza cardiaca e respiratoria, alterato stato di coscienza.

L'instabilità è invece una condizione clinicamente precaria, che necessita un intervento assistenziale di entità variabile in rapporto al grado di instabilità. L'instabilità clinica, per lo più valutata 24 ore prima della dimissione, è fattore prognostico di outcome sfavorevole a 30 giorni, soprattutto in termini di mortalità e riospedalizzazioni.(81) Ad oggi esistono vari strumenti in grado di definire e valutare tale condizione, i principali risultano essere: scala HALM-SIC (scala di instabilità clinica secondo Halm), scala MEWS (Modified Early Warning Score), ViEWS (Vital Early Warning Score) e NEWS (National Early Warning Score).(81)

MEWS, ViEWS e NEWS, in quanto score di allerta precoce (Early Warning Score-EWS), hanno l'obiettivo di fornire una misura riproducibile di quanto un paziente sia a rischio di deterioramento, permettendo così di identificare i pazienti che stanno peggiorando prima che la loro condizione diventi troppo grave. In particolare:

- cogliere il deterioramento delle funzioni fisiologiche prima che i danni d'organo diventino irreparabili;

- definire l'intensità di cura necessaria, fornendo indicazioni precise per la frequenza e la tipologia dell'assistenza nelle diverse sezioni di degenza;

- Il tempestivo riconoscimento della gravità e l'appropriatezza dell'intervento clinico-assistenziale sono tra i fattori che determinano l'esito delle patologie acute.

In letteratura sono annoverati molti altri strumenti per la valutazione dell'instabilità clinica primi fra tutti quelli in uso nelle terapie intensive (SOFA - Sequential Organ Failure Assessment; SAPS - Simplified Acute Physiology Score; APACHE - Acute Physiology And Chronic Health Evaluation, ecc). Si è scelto però di non trattarli in questo documento, che mira alla valutazione e gestione dei soli pazienti di medicina interna.

La complessità assistenziale infermieristica riguarda la gestione di alcuni aspetti di assistenza: la respirazione, la gestione del tubo oro-tracheale, della ventilazione non invasiva, eccetera; i mezzi di accesso venoso centrale o periferico; la nutrizione (enterale, parenterale) e l'idratazione; l'eliminazione urinaria ed intestinale, l'igiene personale, la postura ed il movimento del 
paziente; il riposo ed il sonno, la funzione cardiovascolare, l'ambiente sicuro, l'interazione, la comunicazione con il paziente i famigliari e i caregivers; la loro educazione sanitaria, le medicazioni avanzate ed i farmaci, le procedure diagnostico e terapeutiche, i dispositivi e devices, le modalità di monitoraggio.(81)

\section{L'ipertensione}

La definizione di Ipertensione Arteriosa è arbitraria, in quanto la pressione arteriosa (PA) è una variabile continua normalmente distribuita nella popolazione generale. Può essere espressa come quell'arco di valori pressori per i quali è dimostrato che l'abbassamento ottenuto con la terapia produce benefici in termini di riduzione del rischio di eventi cardiovascolari.

Per la conferma della diagnosi di Ipertensione Arteriosa, le linee guida stabiliscono che i pazienti con valori di pressione clinica uguale o superiore a 140/90 $\mathrm{mmHg}$ in almeno due differenti occasioni, e i pazienti con sospetto clinico anamnestico di Ipertensione Arteriosa, debbano essere sottoposte ad auto misurazione domiciliare della PA e/o monitoraggio ambulatoriale delle 24 ore.

Questo soprattutto per riconoscere quelle particolari condizioni cliniche quali l'ipertensione da camice bianco e l'ipertensione mascherata, per le quali il solo utilizzo corretto delle tre metodiche permette di formulare una diagnosi di certezza.(82)

Gli obiettivi dell'assistenza infermieristica della persona con ipertensione arteriosa sono la riduzione della pressione arteriosa e l'assenza di effetti indesiderati. Per raggiungere tali obiettivi l'infermiere deve insegnare all'assistito ad osservare il regime terapeutico, modificando opportunamente lo stile di vita, assumendo i farmaci secondo le prescrizioni e fissando appuntamenti regolari con il medico per valutare l'efficacia della terapia o identificare e trattare eventuali complicanze dell'ipertensione o della terapia.

I principali modelli di salute alterati e le relative diagnosi infermieristiche (tratte dalle diagnosi NANDANorth American Nursing Diagnosis International) del paziente affetto da ipertensione arteriosa sono:

- Rischio elevato di non adesione, correlato agli effetti indesiderati della terapia prescritta e alla convinzione che non è necessario alcun trattamento in assenza di sintomi.

- Rischio di inefficace autogestione della salute, correlato a insufficiente conoscenza della condizione, delle restrizioni dietetiche, dei farmaci, dei fattori di rischio e dei follow-up.

- Rischio di intolleranza all'attività fisica relativa agli effetti dell'ipertensione sulla vita di tutti i giorni.

Principali problemi collaborativi da affrontare in collaborazione con l'equipe multidisciplinare (Medico medicina generale-Specialista-Dietologo-Infermiere):

- Ipertrofia del ventricolo sinistro;

- Infarto miocardico;
- Insufficienza cardiaca;

- Attacchi ischemici transitori (TIA);

- Accidente cerebrovascolare (infarto o emorragia cerebrale);

- Insufficienza renale;

- Emorragia retinica.

Gli obiettivi principali per l'assistito includono la comprensione della patologia e del relativo trattamento, la partecipazione al programma di autocura e l'assenza di complicanze.

Relativamente al percorso di dimissione, le competenze infermieristiche presenti in letteratura si rifanno tutte ad interventi infermieristici a breve temine:

1) Identificare eventuali fattori che possono predire una non adesione del paziente come:

- Mancanza di conoscenze;

- Non adesione in ospedale;

- Incapacità di percepire la gravità o la cronicità dell'ipertensione;

- Convinzione che la situazione si risolverà.

Si stima infatti che dal 40 al 50\% dei pazienti con ipertensione interrompa il programma di trattamento nel primo anno. Identificare le barriere dell'adesione permette all'infermiere di pianificare interventi che eliminano questi problemi e migliorino la compliance.

2) Mettere in risalto con il paziente che una non adesione può avere conseguenze capaci di mettere in pericolo la sua vita: Segnalare la gravità dell'ipertensione può migliorare l'adesione al trattamento;

3) Sottolineare che l'ipertensione tipicamente non produce sintomi: 1'assenza di sintomi spesso incoraggia la non adesione.

4) Sottolineare l'importanza di un'attività fisica costante e moderata: nell'ipertensione di grado medio l'attività fisica e l'eventuale perdita di peso può rappresentare l'unico trattamento necessario;

5) Ogni volta che sia possibile far partecipare le persone significative per il paziente alla seduta di insegnamento: Anche le persone significative devono comprendere le possibili conseguenze della non adesione alla terapia, in modo che siano incoraggiate ad aiutarlo.

6) Istruire il paziente a controllarsi, o farsi controllare, la pressione arteriosa almeno una volta a settimana, e a tenere un'accurata registrazione dei risultati: I risultati dei controlli settimanali della pressione arteriosa sono necessari per valutare la risposta del paziente ai trattamenti e alle modificazioni dello stile di vita.

7) Spiegare i possibili effetti collaterali dei farmaci antipertensivi, istruire il paziente a consultare il medico per i farmaci alternativi qualora si verifichino alcuni di questi effetti: Una persona che non sperimenta questi effetti collaterali potrebbe essere tentata di interrompere la terapia farmacologica. Indicatori del risultato raggiunto.

Il paziente:

- Esprimerà i sentimenti correlati alla sua adesione al regime terapeutico prescritto; 
- Mostrerà di aver capito l'importanza di un costante esercizio fisico e dell'eventuale perdita di peso;

- Identificherà le fonti di sostegno delle quali trarre aiuto per tale adesione;

- Spiegherà le possibili complicanze di una non adesione.

È importante che l'assistito comprenda i vari aspetti della patologia e il fatto che il cambiamento dello stile di vita e la terapia farmacologica possono controllare l'ipertensione arteriosa. A questo proposito, l'infermiere deve sottolineare il fatto che l'ipertensione arteriosa può essere controllata più che curata.

Migliorare continuità ed aderenza terapeutica non è semplice e richiede un intervento multifattoriale, mirato alle specifiche necessità del paziente. Richiede però un approccio sistemico e un'adeguata organizzazione del lavoro.

Il primo passo per costruire l'alleanza terapeutica con il paziente è costituito dal fornire al paziente (e all'eventuale caregiver) le informazioni necessarie e nell'assicurarne la comprensione.

Prima di prescrivere la terapia antipertensiva è indispensabile verificare quello che pensa il paziente e il suo grado di convinzione. Con rare possibili eccezioni, in caso di dubbi, incertezze, scarsa comprensione da parte del paziente è opportuno rimandare l'inizio della terapia, per un altissimo rischio di precoce abbandono della stessa. Di aiuto potrebbe essere un elenco dei punti da affrontare con il paziente in cui riportare le informazioni necessarie per un'adeguata aderenza al regime terapeutico, affrontando anche in modo schematico i diversi argomenti:(82)

i. Perché è utile ridurre i livelli pressori;

ii. Quali obiettivi pressori (eventualmente in termini di obiettivi intermedi e finali);

iii. Quando e come assumere i farmaci (se necessari schema scritto);

iv. Perché è necessario usare con regolarità i farmaci;

v. Come comportarsi in caso di possibili effetti collaterali;

vi. Quando la prossima visita in ambulatorio;

vii. Se e come controllare la pressione prima della visita di controllo.

Ed inoltre:(82)

- Far presente che la risposta alla terapia è individuale e i livelli pressori possono modificarsi nel tempo (aumento con il tempo, influenza periodi caldi, ecc.) per cui sarà normale effettuare modifiche della terapia nel tempo.

- Verificare se ci sono difficoltà/perplessità nelle modalità di assunzione dei farmaci, anche ponendo domande in modo diretto.

- Verificare la presenza di effetti collaterali reali o presunti.

- Verificare eventuali problemi legati alla presenza di farmaci equivalenti con prezzi differenti (aspetti economici, confusione tra farmaci, ecc.).
Altre raccomandazioni che devono essere fornite al paziente sono:(82)

- Limitare il consumo di sodio e di grassi e, aumentare il consumo di frutta e verdura;

- Svolgere (nel possibile) un'attività fisica regolare;

- Limitare il consumo di sostanza alcoliche e, limitare o eventualmente smettere di fumare, poiché il fumo aumenta il rischio cardiovascolare;

- Ridurre il peso corporeo.

\section{La trombosi venosa profonda}

Il tromboembolismo venoso (TEV) è la patologia cardiovascolare più frequente, dopo la cardiopatia ischemica e l'ictus cerebrale ischemico: risulta infatti al terzo posto tra le cause di morte nella popolazione generale ed al primo posto nei pazienti ospedalizzati. $\mathrm{E}$ in costante aumento per diversi motivi, fra i quali l'allungamento della vita media, l'incremento della chirurgia geriatrica e delle patologie traumatiche.(83)

Dati Istat aggiornati 2014, ultimo anno disponibile (84), mostrano come le malattie del sistema cardiocircolatorio siano la principale causa di morte in Italia. Circa un quarto del totale di queste, viene raggruppato sotto la voce di altre malattie del sistema cardiocircolatorio, in cui troviamo la trombosi venosa profonda (TVP). Il tromboembolismo venoso è quindi la terza malattia cardiovascolare più comune. L'incidenza della TVP in Italia si attesta a circa 1 caso ogni 1000 soggetti per anno nella popolazione generale e varia con l'età, con dei valori minimi durante l'infanzia e adolescenza $(0,005 \%)$ ed un aumento esponenziale fino a 5-25 casi su 1000 nei pazienti tra i 70-80 anni.(83)

La TVP viene considerata una patologia associata ad interventi chirurgici recenti e a traumi, ma nei pazienti non chirurgici ben il $50-70 \%$ ha eventi trombotici sintomatici e il 70-80\% embolie polmonari fatali; anche perché spesso la TVP è asintomatica nei pazienti ospedalizzati in area medica.(85)

Infatti il TEV è rilevabile più frequentemente nel paziente ricoverato per patologie internistiche acute piuttosto che nel paziente chirurgico, dal momento che, in questa tipologia di pazienti, a causa di vari fattori, come la presenza di polipatologie, dalle quali sono solitamente affetti, e della difficoltà di stratificazione del rischio, è stato riscontrato un insufficiente utilizzo della terapia antitrombotica. Sono presenti infatti diversi metodi di stratificazione del rischio, ma risultano in alcuni casi scarsamente utilizzati nella pratica clinica. Circa il $40 \%$ dei pazienti con indicazione alla profilassi con TEV non la riceve. Questo sottoutilizzo, che può essere almeno in parte ascrivibile alla complessità ed eterogeneità dei pazienti in ambito internistico, richiama l'opportunità di sforzi di approfondimento educazionale e di studio.(86)

La SIAPAV (Società Italiana di Angiologia e Patologia Vascolare) definisce la malattia tromboembolica venosa o tromboembolismo venoso, come una 
condizione clinica caratterizzata dall'attivazione patologica della cascata coagulativa nel sistema circolatorio venoso, con la formazione di trombi intra-luminali, che con una certa frequenza possono frammentarsi e/o dislocarsi dalla vena d'origine ed embolizzare nelle arterie polmonari. Solitamente i fenomeni tromboembolici originano nelle vene degli arti inferiori (prevalentemente iliache e pelvi), ma possono insorgere anche in altri distretti venosi, nelle sezioni destre del cuore e nelle arterie polmonari.(83)

Si definisce trombosi venosa profonda (TVP) l'ostruzione completa o parziale di una o più vene del circolo venoso profondo degli arti e/o dell'addome e pelvi.(87)

La TVP è una patologia vascolare potenzialmente severa perché può causare a sua volta un'embolia polmonare (EP), condizione acuta che si verifica quando il coagulo di sangue viaggia attraverso il sistema venoso arrivando fino al sistema circolatorio polmonare, con rischio di morte improvvisa. Questo restringimento del lume può provocare una ridotta ossigenazione ed ipertensione polmonare. Nel tempo, il carico di lavoro imposto sul cuore destro aumenta e contribuisce in maniera negativa sul normale funzionamento cardiaco.(88) La TVP è causa del $90 \%$ dei casi di embolia polmonare.(87)

Inoltre si è visto che fino al 50\% delle persone che hanno sofferto di TVP avrà complicanze a lungo termine generate dal danno provocato dal coagulo alle valvole della vena (definito come insufficienza valvolare) causando la così detta sindrome post trombotica (SPT). Il coagulo rimasto nella vena delle estremità inferiori può inoltre ostacolare il flusso di sangue e portare all'ipertensione venosa, mentre i danni alla vena stessa possono determinare un'infiammazione ed una necrosi dei tessuti venosi che, in alcuni casi, causano i sintomi classici di SPT, come: dolore cronico, edema instabile, pesantezza dell'arto e ulcere della gamba.(88)

I tre fattori che sono stati riconosciuti come concorrenti alla formazione di TEV vengono definiti Triade di Virchow e sono i seguenti:(89)

- inattività e alterazione del flusso sanguigni;

- ipercoagulabilità;

- danni endoteliali o lesioni alle pareti dei vasi sanguigni.

Analizzando la letteratura in materia di nursing al paziente con TVP emergono due principali ambiti di attività, che sono:

- tecnico e di formazione del professionista: la conoscenze scientifiche risultano fondamentali per un'assistenza intelligente, anche perché la chiave per prevenire la TVP è proprio una precoce identificazione dei soggetti a rischio;(89) per questo l'infermiere si avvale dell'utilizzo di scale per l'identificazione, sia dei fattori di rischio di TVP nei pazienti medici ospedalizzati (Padua Prediction Score), che della sindrome post trombotica (Villalta Scale Assessment Symptoms and Clinical Signs Components).
- Il Padua Prediction Score assegna ad ognuno degli 11 fattori di rischio identificati, tra cui cancro attivo, precedenti TVP ad esclusione di trombosi venosa superficiale, riduzione di mobilità, precedente diagnosi di condizioni trombofiliche, trauma o chirurgia recente (meno di un mese), età avanzata (sopra i 70 anni), insufficienza cardiaca e/o respiratoria, IMA o ictus ischemico, infezione acuta e/o patologia reumatologica, obesità (BMI $>30)$, trattamento con terapia ormonale, un punteggio da 1 a 3. Quindi categorizza come pazienti a basso rischio di TVP quelli il cui punteggio finale risulta sotto i 4 , mentre ad elevato quelli con un punteggio maggiore o uguale a 4. Per il calcolo dell'affidabilità, tra i pazienti che non ricevono profilassi, hanno sviluppato TVP 1'11\% di quelli classificati ad alto rischio, rispetto allo $0,3 \%$ di quelli classificati a basso rischio (RR: 32, IC 95\%, [4,1251,0]).(90)

- Lo Scientific and Standardization Committee of the International Society on Thrombosis and Haemostasis identifica invece la scala di Villalta come standard e strumento più appropriato per definire e diagnosticare la severità della SPT.

- La scala descrive segni e sintomi della SPT valutando ogni singolo ITEM in un range da 0 a 4, in cui 0 corrisponde e nessuno mentre 3 a severo; lo score totale può variare da $0 \mathrm{a} 33$, se il punteggio definitivo è tra 0 e 4 , non è presente la SPT, se è situato tra 5 e 9 risulta medio, tra 10 e 14 moderato mentre oltre a 15 è severo; inoltre, questa scala indica la presenza di un ulcera venosa lungo l'arto come identificativo della presenza di SPT, anche senza considerare i punteggi singoli.(91)

- La SPT è considerata appunto una sindrome, dal momento che si manifesta come uno spettro di segni e sintomi legati all'insufficienza venosa cronica, tali sintomi variano da paziente a paziente.

- Inoltre occorre esercitare il proprio operato sulle migliori evidenze per minimizzare il rischio di tromboembolismo venoso.(91), motivo per cui risulta fondamentale avere conoscenze sulla profilassi farmacologica e meccanica.

- La letteratura suggerisce per il paziente medico ricoverato in ospedale con un elevato rischio di trombosi o con un elevato rischio di sanguinamento l'utilizzo della trombo profilassi meccanica con calze elastiche a compressione graduata (grado 2C) o la compressione pneumatica intermittente (grado 2C).(90)

- Infatti, i mezzi di tromboprofilassi meccanica sono raccomandati primariamente nei soggetti ad elevato rischio di sanguinamento (grado 1A) od in aggiunta alla profilassi con anticoagulanti (grado 2A). $(85,89)$

- Le calze elastiche a compressione graduata, dal punto di vista della prevenzione delle complicanze croniche della SPT, non dovrebbero essere utilizzate routinariamente; discorso diverso per quanto 
riguarda la prevenzione dei sintomi, che giustificano il loro utilizzo.(92)

- L'efficacia delle calze a compressione graduata per la prevenzione del SPT è dunque incerta nella letteratura, ma l'applicazione risulta ragionevole per ridurre gonfiore sintomatico nei pazienti con diagnosi di TVP prossimale (grado 2A), escludendo però quelle tipologie di pazienti in cui sono fortemente sconsigliate, ovvero soggetti affetti da insufficienza arteriosa.(93)

- La scelta della terapia anticoagulante, invece, va effettuata in base a diversi fattori, se il paziente non presenta alcun altra patologia, si consiglia una terapia anticoagulante a breve termine (3 mesi) (grado 1B) preferibilmente con dabigatran, rivaroxaban, apixaban o edoxaban (i così detti NAO) rispetto ad una terapia con antagonista della vitamina $\mathrm{K}$ (VKA) (grado 2B); se non è possibile utilizzare i NAO però, la terapia con VKA è comunque preferibile alla somministrazione di eparina a basso peso molecolare (EBPM) (grado 2C).

- Nei casi in cui si ha TVP dell'arto inferiore od EP in presenza di patologia tumorale (la così detta trombosi cancro associata) invece, si preferisce una terapia anticoagulante a breve termine (primi 3 mesi) con EBPM ad una con antagonisti della vitamina K od ai NAO (grado 2C).(92) Inoltre se tollerato, il trattamento iniziale per il paziente con trombosi cancro associata dovrebbe essere di 6 mesi con EBPM (grado 1A).(94)

- Sia nei pazienti con una TEV che hanno basso rischio di sanguinamento (grado 1B), che in quelli che hanno un medio rischio (grado $2 \mathrm{~B}$ ) le linee guida raccomandano una terapia anticoagulante estesa, piuttosto che una di tre mesi; mentre per quelli con alto rischio di sanguinamento meglio optare per una terapia trimestrale(grado 2B).(92)

- L'incoraggiamento alla deambulazione precoce è uno dei punti fondamentali della prevenzione e del trattamento della TVP, dato che stato dimostrato che la contrazione muscolare riduce la stasi venosa ed aiuta le valvole a nido di rondine nella regolazione della corrente sanguigna in senso centripeto. $(83,87,90,95,96)$

- Altro punto cardine è l'idratazione: bisogna favorire l'assunzione di liquidi, a meno che non ci sia una indicazione clinica che richieda una restrizione idrica, poiché la disidratazione rientra nei fattori di rischio della TVP. $(87,95)$

- Educativo/relazionale: i pazienti con TVP, essendo un evento acuto ma con un'evoluzione spesso di cronicità, dovrebbero essere sempre di più coinvolti nelle decisioni di cura. Infatti la sindrome post trombotica è una condizione cronica che si sviluppa in più del $50 \%$ dei pazienti con TVP acuta, incidendo sulla diminuzione della qualità di vita, sull'autonomia personale e sull'aumento dei costi sanitari.(91)

- Queste decisioni dovrebbero basarsi sull'accessi- bilità delle informazioni cliniche, per dare al paziente l'opportunità di fare una scelta consapevole, con l'obiettivo di fornire i migliori strumenti per un reale self-care. In questo realmente l'infermiere gioca un ruolo fondamentale, questa collaborazione è infatti essenziale per migliorare l'aderenza terapeutica, specialmente quando il trattamento farmacologico supera i 3 mesi.(97)

- Lo sviluppo di una reale aderenza terapeutica avviene quando si attiva un'alleanza terapeutica. $\mathrm{La}$ prevenzione post-trombotica e il trattamento dovrebbe iniziare precocemente in ospedale ed estendersi fino alla impostazione ambulatoriale, qualora ne sia necessario.(91)

\section{L'ischemia cerebrale}

Il processo assistenziale, che inizia con l'accesso ai servizi sanitari, è costituito da azioni con priorità differente modulandosi a seconda della fase della malattia. Nella fase acuta la priorità va fornita al triage con valutazione neurologica mirata e all'esecuzione di interventi finalizzati alla prevenzione di complicanze immediate e al controllo delle stesse, limitando l'assistenza ai bisogni essenziali; tra queste vi sono il posizionamento, la gestione della continenza e della pressione, la prevenzione dell'ipertensione potenzialmente lesiva e influenzante ipertensione endocranica.(98) Solo successivamente la priorità andrà a comprendere una valutazione più approfondita al fine di un inquadramento olistico, avviando il percorso di supporto e riabilitazione.

Nella fase acuta, inoltre, gli interventi saranno principalmente di sostituzione, lasciando spazio ed integrandosi all'educazione e alla riabilitazione una volta che le condizioni cliniche lo permettano.

In tutto il percorso, è importante l'utilizzo delle evidenze scientifiche, dunque l'adozione di schede valutative specifiche che permettano di essere riprodotte a distanza fornendo un adeguato confronto clinico (GCS, Cincinnati, NIHSS).

Fase immediata: All'accettazione del malato, risulta particolarmente adeguata la valutazione primaria AB-C-D-E col seguente schema:

A: dev'essere garantita la pervietà delle vie aeree, effettuando una rapida ispezione della posizione della persona e del suo cavo orale, dunque adottare interventi primari di risoluzione quali il posizionamento di una cannula di Guedel;

B: valutazione del respiro, tramite ascolto diretto dei suoni e del flusso di aria ed esecuzione di EGA arterioso; questa tematica è in evoluzione nell'ultimo periodo poiché dalla vecchia indicazione alle somministrazioni routinarie di ossigeno con target di saturazione del $95 \%$ o maggiore, mediante dosi di $2-4 \mathrm{~L} / \mathrm{m}$ via cannule nasali o maschera facciale semplice, si è giunti all'indicazione del trattamento solo in caso di anomalie sintomatiche (dispnea e alterazioni dello 
stato di coscienza) o oggettivamente gravi (sat $<90 \%$ ). Fondamentale è in ogni caso mantenere un attivo monitoraggio della saturimetria e del posizionamento della persona, quando appropriato, con schienale alzato piuttosto che supino, al fine del miglioramento della capacità di ventilazione e della riduzione del rischio di aspirazione;(98)

C: valutazione del circolo, del colorito, della temperatura, della pressione arteriosa e della glicemia, esecuzione di ECG, posizionamento di accesso venoso di grosso calibro, esecuzione di prelievi ematici;

D: valutazione dello stato neurologico generale, dunque dello stato di coscienza (preferibilmente adottando una scheda validata quale Glasgow Coma Scale o AVPU), dei principali deficit neurologici (simmetria del volto e del corpo, forza e movimento degli arti);

E: esame obiettivo testa-piedi.(99)

Dopo le prime valutazioni prioritarie e le prime gestioni, è possibile ampliare l'accertamento ricercando i possibili bisogni di assistenza infermieristica direttamente correlati all'ictus, richiedenti intervento diretto ed eventuale collaborazione con altre figure professionali, quali:

- Compromissione della mobilità correlata al deficit con ipostenia, flaccidità o spasticità di una parte corporea più o meno vasta, oppure per la perdita di equilibrio o coordinazione;

- Dolore posturale legato alla difficoltà di controllo di parte del proprio corpo e quindi al cambio posturale;

- Deficit nella cura del sé correlato ai deficit neurologici dell'ictus (motori e cognitivi);

- Disturbo della percezione sensoriale correlato al danno dell'ictus, causante ridotta funzionalità

- Compromissione della deglutizione correlato al danno dell'ictus direttamente sul processo di deglutizione oppure sullo stato di coscienza;

- Incontinenza urinaria correlata al danno diretto dell'ictus causante una riduzione della percezione o del controllo autonomo oppure ad uno stato di coscienza alterato;

- Ritenzione urinaria acuta correlata al danno diretto dell'ictus causante una riduzione della percezione o del controllo autonomo oppure ad uno stato di coscienza alterato;

- Incontinenza fecale correlata al danno diretto dell'ictus causante una riduzione della percezione o del controllo autonomo oppure ad uno stato di coscienza alterato;

- Compromissione della comunicazione verbale correlata al danno dell'ictus nella formulazione della frase, nella sua produzione meccanica, nella ricezione/comprensione oppure di tutto il processo;

- Rischio di compromissione dell'integrità cutanea correlata al deficit motorio, percettivo sensoriale ed eventuale alterazione dello stato di coscienza;
- Rischio di inefficace protezione delle vie aeree e degli scambi gassosi.

In base ai deficit riscontrati, i principali aspetti tematici di intervento assistenziale al fine di una presa in carico globale saranno dunque:

- Gestione della postura: sono necessari periodici cambi posturali per compensare il deficit della persona nel provvedervi in autonomia, consapevolmente o inconsapevolmente. Gli arti e le parti del corpo colpite da plegia/paralisi devono essere sempre considerati in primo piano, poiché in caso di danno neurologico la muscolatura flessoria andrà a prevalere su quella estensoria. Gli interventi da attuare sono quelli del mantenimento della mano leggermente supina con dita a riposo senza chiusura ferrata (complicanza potenziale nella spasticità a lungo termine), della spalla supportata da un cuscino evitando l'adduzione, del braccio e della gamba estesi, adottando eventualmente un archetto per prevenire il piede equino.

Una buona gestione posturale, se ben pianificata e attuata, concorre a limitare le potenziali complicanze del dolore posturale, dell'edema localizzato, della polmonite ab ingestis, della TVP e delle lesioni da pressione. $(98,100)$ In questo contesto, la fase riabilitativa risulta indicata già nelle prime 24 ore dall'ictus qualora non sussistano controindicazioni; essa verrà attuata con movimenti attivi e passivi più volte al giorno, anche mediante l'utilizzo dell'emisoma indenne per sollecitare quello controlaterale, e con la mobilizzazione precoce e progressiva mediante assunzione di posture gradualmente più verticali: supino, semi Fowler, seduto a letto e successivamente in carrozzina con graduale ripresa alla deambulazione.(101)

- Prevenzione della compromissione dell'integrità cutanea: i cambi posturali periodici, con intervallo da 1 a 4 ore, associati ad interventi di supporto quali il posizionamento di materasso ad aria o ad acqua, oltre che all'igiene regolare e al mantenimento di cute detersa e asciutta, permettono di ridurre il più possibile l'insorgenza di lesioni da decubito complicanti il decorso.

- Valutazione della deglutizione e gestione dell'alimentazione: le evidenze mostrano una necessità alla valutazione precoce della disfagia entro le $24 \mathrm{~h}$ successive al periodo acuto/critico, in modo da individuarla, monitorarla e prevenirne le complicanze connesse. In questo modo è possibile applicare adeguamenti della consistenza di liquidi e solidi ed eventualmente permettere di iniziare le somministrazioni per bocca, tra cui dei medicinali, fatto non prima consigliato.(98)

La valutazione della presenza di disfagia o del rischio di insorgenza è attuabile seguendo il test del cucchiaino/standardized bedside swallowing assessment; nel caso di adeguatezza alla valutazione preliminare (stato di vigilanza con 
collaborazione negli ordini semplici, capacità alla tosse, alla deglutizione della saliva e al mantenimento in asse di tronco e capo), si procede a somministrare tre volte una quantità precisa e ad ogni fase più consistente di acqua $(5 \mathrm{~mL}$ le prime tre somministrazioni, e $50 \mathrm{~mL}$ le successive tre), valutando di passo in passo la presenza di segnali negativi come alterazioni foniche della voce (quali gorgoglio, raucedine oppure maggiore profondità), comparsa di tosse, rallentamento della deglutizione con continuo rimaneggiamento nel cavo orale, mancato riconoscimento dell'acqua o ancora persistenza di materiale nel cavo orale.

$\mathrm{Nel}$ caso in cui il test risulti positivo per disfagia in un passo, sono indicati degli adeguamenti, procedendo al medesimo test con acqua addensata oppure gelificata. I risultati possono essere di deglutizione fisiologica, disfagia lieve con voce gorgogliante dopo la deglutizione di acqua, disfagia media con voce gorgogliante e tosse dopo la deglutizione di acqua e disfagia severa quando presenta tosse severa alla deglutizione di liquidi e solidi.(102)

Nel caso di disfagia accertata sono dunque da applicare correzioni dell'idratazione e alimentazione per prevenire l'aspirazione, pur garantendo la somministrazione di liquidi, cibo e terapia; gli adeguamenti possibili sono quattro:

- dieta omogeneizzata,

- dieta semi-solida,

- dieta solida morbida,

- dieta regolare con addensamento/gelificazione dei liquidi.

Alcune accortezze ulteriori permettono di limitare i rischi mantenendo una buona qualità, quali evitare le doppie consistenze, evitare i cibi friabili o filacciosi e scegliere le formulazioni farmacologiche tritabili/in forma soluzione orale ecc.

Nel caso in cui non siano possibili adeguamenti nella consistenza, è opportuno prendere in considerazione interventi sostitutivi in collaborazione col medico, quali il supporto nutrizionale parenterale, il posizionamento di $S N G$ per i primi tempi e $P E G$ per la fase cronica consentendo nutrizione enterale artificiale, oltre che eventuali supporti dietetici proteici e calorici per limitare la malnutrizione.

La tecnica di assunzione del pasto e idratazione è fondamentale che sia correttamente applicata per limitare i rischi e le alterazioni di deglutizione; è dunque opportuno mantenere il contatto visivo in un ambiente tranquillo lasciando il giusto tempo per il malato, fornendo feedback e incitazioni per la corretta deglutizione.

In ultima analisi, la deglutizione, sia nella forma fisiologica che patologica, va monitorata nel tempo in modo da riconoscere precocemente eventuali evoluzioni da trattare con adeguamenti del caso. La sua corretta gestione, infatti, permette di limitare i rischi connessi di inalazione, polmonite, disidratazione e malnutrizione.(100)

- Gestione e monitoraggio pressorio: rilevazioni regolari e mantenimento di un profilo pressorio, attuando interventi di controllo farmacologico indicati dal medico in caso di valori elevati fuori dai range preposti permettono di ridurre il rischio evolutivo del danno e della comparsa di complicanze, specialmente durante la fase critica e post critica.(98)

- Gestione della normale volemia: è indicato il mantenimento di un circolo funzionale, valutato mediante bilancio delle entrate e delle uscite, pressione arteriosa, refill capillare e diuresi, intervenendo con infusioni endovenose limitando le soluzioni glucosate. $(98,101)$

- Monitoraggio della glicemia e gestione delle alterazioni (ipo/iperglicemia): questo passaggio, da eseguire già nella fase critica, permette la discriminazione da condizioni di ipoglicemia aventi sintomatologia simile all'ictus e predisponenti al danno, oltre che al mantenimento di una condizione fisiologica; è opportuno dunque attuare interventi per mantenere la glicemia nei range, superiore a $80 \mathrm{mg} / \mathrm{dL}$ e inferiore a 200 $\mathrm{mg} / \mathrm{dL} .(98,101)$

- Gestione della funzionalità vescicale e intestinale: è opportuno prendere in considerazione le potenziali alterazioni della continenza in persone con qualunque danno neurologico da ictus. È infatti possibile sia un'alterazione diretta, con incontinenza urinaria o fecale causata dalla perdita di controllo sfinteriale, oppure per alterazione funzionale quale può essere l'insorgenza di dipendenza nelle attività e l'incapacità alla comunicazione della necessità. Nella fase cronica, invece, è possibile anche l'instaurazione di stipsi, principalmente legata all'allettamento.

Per quanto riguarda l'apparato urinario, è altresì possibile l'instaurazione di ritenzione urinaria acuta causata da ipertono detrusoriale.

In entrambe le condizioni è opportuna una valutazione del modello di eliminazione, per attuare monitoraggi appropriati e interventi specifici, avvalendosi eventualmente dell'ecografo per la vescica, del cateterismo vescicale a dimora o intermittente, di urocondom o altri presidi di continenza urinaria, scelte prese in collaborazione con il medico. $(98,100)$

- Gestione della cura del sé (igiene, vestizione, cura del proprio aspetto e dell'uso del bagno): la cura del sé è un aspetto ampio, cui provvedere con supervisione, supporto o sostituzione parziale/totale qualora questa sia deficitaria; è opportuno mantenere sempre alto il livello di conoscenza, istruzione ed educazione sullo svolgimento della tecnica e la presenza di presidi di aiuto. Specialmente nei pazienti con disfagia è importante l'igiene del cavo orale minuziosa, in 
modo da permettere una corretta sensibilità al cibo e al gusto e ridurre la persistenza di materiale nello stesso, così da limitare il rischio aspirazione. $(101,102)$

- Gestione della comunicazione: nel caso di alterazioni del modello comunicativo, dunque disartria, disfasia o afasia, è importante attuare interventi in acuto per il mantenimento di una comunicazione funzionale sui principali bisogni acuti e per l'adozione di un modello efficace riabilitativo.

$\mathrm{La}$ riabilitazione indicata in letteratura per la disartria propone interventi comportamentali che permettono di aumentare la forza, la velocità e la capacità di utilizzo di quei muscoli intaccati, tra cui impacchi di acqua fredda, agopuntura o frizioni del viso con la finalità di sollecitarli nel loro lavoro; in alternativa è possibile adattare meccanismi di compensazione quali il rallentamento, l'accentuazione dei movimenti necessari per la produzione della parola, oppure l'adozione di vie comunicative di supporto come carte di dialogo e voce artificiale computerizzata.(103)

Per quanto riguarda disfasia e afasia i possibili interventi di riabilitazione prevedono il supporto logopedico mirato, dove l'obiettivo è potenziare la funzionalità comunicativa, il linguaggio ricettivo e il linguaggio espressivo; a tali finalità è necessario attuare interventi di supporto e di educazione mirati, con personale esperto nell'ambito. Tra questi vi sono la stimolazione alla comunicazione e al contatto sociale, così da acquisire consapevolezza e fornire supporto per il miglioramento, oppure tecniche per il miglioramento dei singoli aspetti del linguaggio ricettivo (la lettura, l'associazione di gesti a significati e suoni) e di quello espressivo (mediante tecniche che permettano la coordinazione corretta e formulazione concreta del pensiero, o strumenti di supporto quali le tavole che riportano i principali bisogni o le principali frasi oppure i dispositivi elettronici).(100,104) Errori comuni da evitare sono quelli di completare le frasi dell'assistito. È importante inoltre usare frasi brevi, chiare e concrete, mantenendo il contatto visivo e ripetendo il nome degli oggetti presi o delle azioni compiute, lasciando il tempo richiesto alla persona per elaborare il pensiero e ricevere i giusti input.(100) Particolarmente rilevante risulta l'educazione dei famigliari e caregiver nella comprensione del problema e nella sua gestione (mediante i suddetti supporti e accorgimenti), oltre che alla considerazione di eventuali disturbi di personalità e pensiero legati alla patologia cui rispondere senza aggressività bensì con comprensione.

- Monitoraggio della temperatura: le evidenze indicano necessità alla valutazione ogni $4 \mathrm{~h}$ nelle prime $72 \mathrm{~h}$ oltre che all'intervento con somministrazione di paracetamolo se questa dovesse risultare $>37,5^{\circ} \mathrm{C} .(98)$

- Gestione dell'ipossia/protezione delle vie aeree: mantenimento e monitoraggio degli aspetti correttivi/di supporto suddetti tra gli interventi prioritari facenti parte di A e B del processo ABCDE.

Nella relazione malato infermiere sono presenti molti momenti educativi dove le specifiche competenze per informare, istruire ed educare le persone, aiutano ad adottare stili di vita sani oppure ad acquisire capacità di autocura. L'educazione ripone dunque un ruolo molto influente sullo stato di salute del malato e sulle sue possibilità future; per questo motivo deve iniziare con l'accesso ai servizi sanitari, accompagnando ogni azione che si esegue con o per il malato. Esso rientra nel percorso riabilitativo che vede una pianificazione di interventi multidisciplinari calati nella realtà individuale della persona, dunque in base al suo grado di autonomia iniziale, alle sue capacità precedenti e al suo contesto socio famigliare.(102)

Gli obiettivi del progetto educativo saranno quelli del:

- raggiungimento del maggior grado di conoscenza del problema/patologia, dei fattori di rischio per ricadute o recidive e dei comportamenti adeguati.(105)

- raggiungimento del maggior grado di autosufficienza possibile e, ove possibile, del raggiungimento di capacità che permettano di superare la propria disabilità/disagio. $(100,101)$

Tutto ciò è raggiungibile seguendo un percorso che preveda inizialmente attività semplici, fondamentali e prevalentemente assistenziali, per poi aumentare di complessità ed effetto terapeutico, con tanti piccoli obiettivi realistici e concreti, a breve, medio e lungo termine.

Qualunque intervento educativo pianificato e strutturato, porterà a dei risultati, con maggiore consapevolezza specialmente nella popolazione più giovane con meno comorbidità.

Al fine di una corretta pianificazione riabilitativa, è fondamentale utilizzare strumenti validati e universali al fine di uniformare la valutazione della funzionalità globale, mantenendo un confronto tra la fase iniziale e quelle successive, quali possono essere la scheda FIM o Barthel index.

Gli aspetti da includere, con un processo di informazione, istruzione o addestramento ed educazione attuato in ogni contesto di bisogno assistenziale, sono:

- L'autocura della persona: il counselling aiuta la persona ad utilizzare le proprie risorse per raggiungere il miglioramento, accompagnando il processo riabilitativo non appena le condizioni della persona lo consentano (stato di attenzione sufficiente e capacità a mantenere posture consone). È importante in un primo momento sollecitare la persona a considerare il lato colpito dall'ictus, 
utilizzando le proprie funzionalità residue per quelle attività attuabili con una sola mano, quali l'igiene, la vestizione, i movimenti e anche per il mantenimento di una buona elasticità controlaterale (prevenendo spasticità e contratture muscolari). Alcuni accorgimenti che si possono insegnare, permettono una maggiore semplicità di esecuzione, quali uno specchio, il mantenimento degli oggetti a portata del lato sano, l'utilizzo di vestiti comodi evitando i bottoni e iniziando ad indossarli dal lato colpito. Possono essere consigliati anche alcuni ausili e adeguamenti per l'ambiente domestico, quali l'utilizzo di utensili con impugnatura ampia, spugne con manico lungo, bastoni/girelli/sedie a rotelle per il movimento, l'installazione di barre di sostegno nel bagno, di un montascale, di una doccia al posto della vasca per lavarsi oppure di un seggiolino nella stessa.

Per quanto riguarda la postura da assumere al fine dell'alimentazione, si deve insegnare fin dalla fase acuta al mantenimento del tronco eretto e in asse, con il capo piegato in avanti e adoperando eventuali protesi dentarie presenti.(100)

- Le possibili complicanze:

- L'emineglect richiede interventi educativi e riabilitativi specifici, quali posizionare tutti gli stimoli e oggetti nel campo visivo indenne nella fase acuta, andando poi a sollecitare e attirando l'attenzione verso l'altro lato col progredire del tempo.

- La disfagia, invece, richiede che vengano attuati interventi di educazione alla corretta preparazione e somministrazione del pasto, nonché al corretto riconoscimento dei problemi possibili col progredire del tempo.

- Le lesioni da decubito richiedono specifiche competenze gestionali, dunque un supporto professionale per il monitoraggio e la gestione; di competenza della famiglia e del caregiver risulta tuttavia la mobilizzazione costante e la prevenzione, nonché la corretta igiene e detersione cutanea.

- L'autocura farmacologica: è fondamentale che il malato e il caregiver conoscano le giuste modalità di assunzione dei farmaci, oltre che le tempistiche, le modalità di reperimento e le principali complicanze legate alla non assunzione o al sovraddosaggio.

- La conoscenza del problema, dei fattori di rischio e dei comportamenti compensatori/dello stile di vita sano: l'informazione mirata a spiegare la patologia, i fattori di rischio specifici, i possibili comportamenti per limitarli e il razionale degli interventi apportati, può avere dei risvolti positivi sulla compliance terapeutica/riabilitativa, sulla qualità di vita, sulla capacità di indipendenza e sulla capacità di partecipazione sociale.(106)

In tutto il percorso riabilitativo ed educativo, va riposta molta importanza nel coinvolgimento dei famigliari e dei caregiver nel percorso assistenziale e di cura; questi, infatti, permettono una maggior comprensione del malato e vi forniscono fiducia, portando ad un potenziale miglioramento degli outcomes e della qualità di vita.(107) L'attenzione su essi riposta, tuttavia, non deve risultare unicamente in vista del malato, bensì anche nei loro confronti, dunque della loro qualità di vita, potenzialmente colpita negativamente dall'evento; in questo senso è importante mantenere alto il loro grado di comprensione della patologia e dei risvolti che essa può avere sulla mentalità, affettività e capacità motoria del malato, adottando tecniche di supporto e informazione sui servizi socio territoriali.

La fase riabilitativa deve essere intrapresa il più precocemente possibile e già considerata nelle 48 ore dall'ictus, scegliendo dunque il percorso più appropriato una volta terminata la fase acuta/critica. Il percorso prevede dunque l'ingresso in stroke unit con trasferimento ad unità di neurologia il più presto possibile (una volta che lo consentono le condizioni cliniche) e il passaggio in unità riabilitativa entro 30 giorni per migliorare gli outcomes. Le strutture riabilitative, intensive, non intensive, ed estensive vengono scelte in base alla gravità della disabilità conseguente ad ictus, indirizzando dunque a quelle intensive solamente il grado moderato-severo di disabilità.

Il percorso di dimissione, aspetto cruciale per il malato, va pianificato tenendo in considerazione le disabilità residue, la quantità assistenziale richiesta e scegliendo infine il contesto con maggiore adeguatezza:

- rientro al domicilio: è possibile per quelle persone con capacità di autogestione e autocura che non richiedono supporto e assistenza esterna al nucleo familiare;

- rientro al domicilio con attivazione di servizi (bassa, media o alta intensità): indicato nel caso la disabilità sia lieve o moderata e il contesto domestico richieda solo piccoli interventi di adeguamento strutturale, nonché interventi esterni di assistenza e presa in carico mediante servizi territoriali, come ADI, coordinati dal MMG;

- ingresso in strutture residenziali, post acute o lungodegenze: le RSA, le unità per post acuti o lungodegenze territoriali, sono la scelta prioritaria per quei pazienti con grave disabilità, scarsa possibilità di recupero e necessità assistenziali elevate non gestibili a domicilio.

Qualunque sia il contesto scelto, va riposta molta importanza anche nel reinserimento sociale, al fine di promuovere l'attività, l'aderenza e il benessere.

In tutto il percorso assistenziale, compreso quello riabilitativo, l'infermiere collabora con le figure professionali che si intrecciano, monitorando la risposta del malato ai vari programmi ed attuando eventuali tecniche indicate dagli altri professionisti, in 
modo da fortificare il risultato e gestire nel miglior modo il deficit neurologico.(100,101,102)

La creazione di una rete assistenziale e la presa in carico olistica della persona nel vario contesto (domiciliare, ambulatoriale o residenziale), permette di proseguire con educazione e monitoraggio dello stato di salute, nonché verifica della perseveranza nei comportamenti a rischio modificabili.

I controlli follow-up prevedono l'esecuzione di esami strumentali/di laboratorio che monitorino nel tempo la problematica e di visite specialistiche che riconoscano eventuali evoluzioni nelle complicanze connesse (quali la disfagia, le lesioni da decubito, ecc.).

Nonostante l'importanza della tematica, non sono attualmente presenti evidenze scientifiche che permettano di pianificare la giusta frequenza di follow-up, per cui i piani risentono delle risorse territoriali; in ogni caso è indicato un follow-up regolare e al bisogno ogni qualvolta il caregiver individui segni e sintomi di possibili complicanze oppure si trovi in difficoltà gestionale.

\section{La disfagia}

Molteplici rapporti epidemiologici dimostrano come la prevalenza della disfagia sia più comune tra la popolazione anziana, direttamente proporzionale all'aumentare dell'età con un impatto importante su morbidità, tempi di ospedalizzazione e costi sanitari. La prevalenza nella popolazione generale viene stimata intorno al $20 \%$, con aumenti significativi in popolazioni specifiche come quella degli anziani istituzionalizzati, i portatori di esiti di interventistica demolitiva del distretto testa-collo, nei soggetti affetti da morbo di Parkinson, nei traumatizzati cranici e nei pazienti con ictus cerebri. $(108,109)$

Secondo l'American Speech-Language-Hearing Association (ASHA) problemi di deglutizione si riscontrano nel $13-14 \%$ dei pazienti ospedalizzati per il trattamento di patologie di tipo acuto, nel $30-35 \%$ dei soggetti ammessi in centri riabilitativi, nel $40-50 \%$ di quelli ricoverati in strutture di lungodegenza, $(108$, 110) mentre il problema risulta presente nel 13\%-38\% degli anziani che vivono al domicilio.(111)

Nonostante i numerosi studi analizzati, definire l'esatta prevalenza e incidenza della disfagia è difficile da riportare, infatti varia in base ai disturbi di salute concomitanti, alla popolazione indagata e allo strumento diagnostico utilizzato.

Il disturbo deglutitorio è una condizione assai pervasiva e potenzialmente mortale che può manifestarsi con due modalità principali:

- Disfagia ovvero un'alterazione della deglutizione che non si configura come una malattia, dunque con eziologia, patogenesi ed evoluzione proprie, bensì come un segno o un sintomo di una situazione clinica, a seconda che venga rispettivamente riconosciuta dal clinico o riferita dal paziente;

- Presbifagia ovvero un rallentamento della deglutizione, possibile sia nella fase orofaringea che in quella esofagea, principalmente legato all'invecchiamento, ma potenzialmente presente in persone altrimenti sane. Tale fenomeno si presenta in conseguenza ad un'acquisita riduzione di efficienza neuromotoria e muscolare.(112)

La disfagia è associata ad un ampio numero di condizioni neurologiche come trauma cranico, ictus, sclerosi laterale amiotrofica, morbo di Parkinson, demenza, miastenia grave, sclerosi multipla e malattie del motoneurone. Inoltre, come già detto, può manifestarsi in soggetti anziani sani a causa di modificazioni dell'orofaringe dovute all'età, in soggetti con un deficit neurologico subclinico e può dipendere dall'uso di alcuni farmaci. $(110,113)$

L'Organizzazione Mondiale di Gastroenterologia suddivide la disfagia in due categorie principali: disfagia orofaringea, che si manifesta con la difficoltà ad innescare il meccanismo deglutitorio e disfagia esofagea, dove invece il paziente manifesta la sensazione soggettiva di ostacolo al passaggio del bolo. Quando si sospetta o viene diagnosticata la disfagia, è importante stabilire quale parte del tratto gastrointestinale superiore sia coinvolto e l'eziologia scatenante. È fondamentale stabilire inoltre se la difficoltà di deglutizione si verifica per i liquidi, i solidi o per entrambi.(110)

Come conseguenza della disfagia si può osservare un deficit dell'intake alimentare con progressivo calo ponderale, carenze di vitamine e minerali, disidratazione, fino a malnutrizione marcata. Le carenze nutritive espone i soggetti ad ulteriori rischi per la salute, come il rallentamento dei processi di cicatrizzazione, una maggiore predisposizione alle infezioni, disturbi delle funzioni mentali e fisiche. $(108,111)$

Un'altra complicanza della disfagia è il rischio di aspirazione con conseguente polmonite ab ingestis e in alcuni casi estremi la morte per soffocamento. La polmonite ab ingestis è un quadro infettivo del parenchima polmonare, che trova nel passaggio delle sostanze alimentari l'elemento scatenante, in quanto le sostanze alimentari stesse fungono da pabulum per i microrganismi patogeni, specie dove i meccanismi protettivi della tosse nonché dal sistema immunitario sono deficitari.(111,114) La disfagia può essere causa di altri disturbi polmonari, come la comparsa di fibrosi polmonare in caso di microaspirazioni croniche o, peggio, di sindrome da distress respiratorio acuto (ARDS, acute respiratory distress syndrome) in caso di aspirazione di materiale di provenienza gastrica refluito nelle alte vie aeree.(111)

Inoltre perdere la funzione della deglutizione non significa semplicemente ridurre l'apporto nutrizionale necessario alla vita, ma significa anche perdere il piacere del cibo con conseguente deflessione del tono dell'umore e riduzione della qualità della vita.(115)

E' molto importante che gli infermieri sappiano riconoscere i fattori di rischio e i primi segni di disfagia, che facciano, quando necessario, la richieste per ulte- 
riori valutazioni e che stilino un piano assistenziale ad hoc sul singolo paziente.

L'infermiere che opera nel setting della Medicina Interna e nello specifico che si trova a dover prestare assistenza al soggetto a rischio o con la sintomatologia disfagica dovrà essere in grado di mettere in atto interventi tecnici e interventi educativi-riabilitativi, rivolti non solo ai pazienti in questione, ma anche ai caregivers e a tutto il personale che si occupa di prestare assistenza.

Nell'ambito tecnico l'infermiere dovrà essere in grado di:

- Individuare i pazienti considerati a rischio disfagia ed eseguire lo screening deglutitorio. Tutti i pazienti affetti da stroke devono essere sottoposti a screening per la disfagia entro 24 ore dall'evento e comunque prima di iniziare l'assunzione del pasto per bocca. Inoltre, dovrebbe essere ripetuto ad ogni cambiamento delle condizioni neurologiche, cliniche e dello stato di deglutizione.(116, 117) Tutti i pazienti con sospetta disfagia o con una persistente perdita di peso e ricorrenti infezioni toraciche devono essere avviati verso un immediato percorso di screening prima di iniziare la normale assunzione del pasto.(116) È raccomandata una valutazione tramite il Bedside Swallowing Assessment (BSA) $(109,110,114,116,117)$ test da eseguire in un ambiente privo di distrazioni, mantenendo il paziente in posizione seduta o semiseduta, e fornendo il giusto tempo per la deglutizione; essa si costituisce da 3 fasi combinate tra di loro:

Fase $1^{\circ}$ : valutazione preliminare che comprende il livello di coscienza, il controllo del tronco e della testa, l'assenza di respiro patologico, la presenza del riflesso della tosse, presenza del gag reflex, la qualità della voce e la capacità di deglutire la saliva; qualunque alterazione in questa fase, rappresenta una controindicazione al proseguo del test.

Fase $2^{\circ}$ : Burke's Three-oz Water Swallow Test (WST), assunzione di $5 \mathrm{ml}$ di acqua per 3 volte tramite l'uso di un cucchiaino da caffè; ad ogni somministrazione si valuta l'assenza di segni di inalazione. Del WST Esistono due varianti: il WST sensibilizzato con pulsossimetro (reperto patologico: caduta della saturazione di ossigeno maggiore del $2 \%$ dopo la deglutizione di almeno $10 \mathrm{ml}$ di acqua) e il WST sensibilizzato con auscultazione.

Fase $3^{\circ}$ : Se la deglutizione allo stadio 2 è risultata normale, si procede a fornire $50 \mathrm{ml}$ di acqua da un bicchiere, a piccoli sorsi, controllando la comparsa di segni di ristagno faringeo, tosse, gorgoglio o alterazioni foniche. Qualora l'esame non fosse completamente negativo, si procede alla valutazione con consistenza diversa, ovvero con acqua gelificata, seguendo i medesimi passaggi e valutando gli indicatori patologici succitati.

Per una più ampia valutazione della sintomatologia associata all'atto deglutitorio è consigliato l'uso di questionario self-report come Eat 10 o il Sydney Swallowing Questionnaire.(113)

- Interpretazione dello screening e classificazione della disfagia per gravità: assente (deglutizione corretta), lieve (comparsa di voce roca o gorgogliante), moderata (comparsa di tosse, voce roca e gorgogliante), severa (comparsa di tosse severa e voce gorgogliante).

In presenza di sospetta aspirazione richiedere un ulteriore valutazione a figure con maggiori competenze d'ambito (logopedista, foniatra, neurologo, dietista, radiologo) che oltre ad un attento esame clinico eseguiranno valutazioni strumentali specifiche come la videofluoroscopia (VFS) o la valutazione della deglutizione con fibroscopio flessibile (FEES).(116)

- Monitorare il paziente durante l'assunzione del pasto, riconoscendo prontamente i segni e sintomi di disfagia e di aspirazione tracheo-bronchiale: ristagni faringei o blocco del cibo ai lati delle guance; deglutizioni multiple per ogni singolo boccone; sensazione di bolo laringeo; tosse persistente o soffocamento durante e nei successivi 2-3 minuti dall'assunzione del pasto; voce gorgogliante o disfonia dopo la deglutizione; rigurgito nasale; presenza di febbre senza alcuna causa evidente; rumori respiratori avventizi all'esame auscultatorio; improvvisa caduta della Sat02; cambiamenti della frequenza respiratoria e frequenti infezioni toraciche.(113)

- Collaborare alla fase di screening nutrizionale, che deve avvenire entro 24- 48 ore dal ricovero in reparto ed ha l'obiettivo di prevenire le complicanze della disidratazione e della malnutrizione.(116, 117) Lo screening nutrizionale deve comprendere: gli indici antropometrici (peso, altezza e circonferenza vita) per il calcolo dell'IMC (Indice di Massa Corporea); indici biochimici (albuminemia, prealbumina, transferrina, proteine legate al reticolo, la conta linfocitaria); l'anamnesi alimentare; la stima del decremento ponderale non intenzionale negli ultimi tre mesi e le patologie associate.(117) In aggiunta, occorre controllare il peso almeno ogni settimana per tutta la durata del ricovero: una riduzione del $2 \%$ rispetto alla settimana precedente è indice di una nutrizione insufficiente. La valutazione deve anche comprendere la capacità delle persona a mangiare da sola, l'appetito e il controllo periodico degli alimenti consumati che va riportato in modo routinario all'interno della cartella infermieristica. $(108,116)$

Nella valutazione del rischio nutrizionale le linee guida consigliano di utilizzare il Nutritional Risk Screening (NRS) o il Malinutrition Universal Screening Tool (MUST).(117,118) In presenza di malnutrizione proteico-energetica garantire l'assunzione di integratori alimentare.(115, 118, 119)

- Assicurarsi che struttura, consistenza e tipo di cibi solidi e liquidi siano consumati come da 
prescrizione. A tal riguardo la consistenza dei liquidi deve essere aumentata utilizzando gli addensanti in commercio (fogli di gelatina, fecola, farina) mentre gli alimenti vanno scelti in base alla consistenza purea, tritata, morbida e normale. $(111,115)$

- Mettere in atto gli aggiustamenti posturali del capo, collo e tronco per facilitare la deglutizione e ridurre il rischio di aspirazione. E' importante precisare che non esiste una posizione ideale da raccomandare a tutti i soggetti disfagici. Le raccomandazioni generali sostengono la posizione seduta a $90^{\circ}$ con i segmenti corporei sostenuti e bene allineati, il capo flesso in avanti e il mento quasi a toccare il manubrio sternale. Mantenere tale posizione per almeno 30 minuti dopo il pasto. A carico della testa, a seconda della causa scatenante, è invece indicata la flessione anteriore, flessione laterale verso il lato sano, rotazione verso il lato leso, estensione e decubito laterale.(113,118,119)

La flessione anteriore del capo, con il mento rivolto verso il basso quasi a toccare lo sterno, è la postura maggiormente usata per semplicità di esecuzione e per il massimo livello di evidenza (grading A). In generale le altre posture o manovre compensatorie hanno un livello di evidenza pari a grading B.(108)

- Stimolare in paziente all'esecuzione delle manovre deglutitore, prescritte dal logopedista o dal foniatra in base al livello di compliance del paziente: doppia deglutizione, manovra di Mendelsohn, deglutizione forzata, sopraglottica e super-sopraglottica. $(108,109,113,115)$

- Valutare la possibilità e la fattibilità del fornire l'indicazione free water, ovvero il consenso all'assunzione di acqua, previa rigorosa igiene orale, nei pazienti con disfagia lieve e poca compliance per i liquidi a consistenza modificata. $(111,119)$

- Assicurare la somministrazione sicura dei farmaci evitando di proporli in una forma diversa da quella per cui è stato introdotto in commercio poiché si rischia di alterarne la concentrazione e la velocità di assorbimento, con conseguente sovradosaggio o sottodosaggio. La triturazione dei farmaci è una pratica che dovrebbe essere evitata, in particolare l'infermiere prima di modificare la forma del farmaco che somministra dovrebbe consultare l'elenco dei farmaci do not crush stilato dall'Istitute for Safe Medicacation Practice aggiornato al 2015, tra questi si segnalano le formulazioni gastroresistenti e a rilascio prolungato.(113)

- Osservare e favorire, in base al grado di abilità e di collaborazione del paziente, l'igiene orale quotidiana. Una corretta igiene orale è importante per evitare la proliferazione di microrganismi patogeni, per prevenire disturbi locali e per ridurre il rischio di polmonite. $(111,116)$ La spazzolatura meccanica dei denti deve essere eseguita prima e dopo l'assunzione del pasto, mentre la pulizia delle protesi dentarie deve essere eseguita almeno 2 volte/die. L'uso di collutori a base di clorexidina allo $0,05 \%$ (priva di base alcolica) per 2 volte/die riduce ulteriormente il rischio di polmonite da aspirazione.(113)

- Nei casi di disfagia grave assicurarsi che la prescrizione nulla per bocca venga garantita e su indicazione medica avviare la terapia nutrizionale artificiale.(111) La terapia nutrizionale di prima scelta è rappresentata dalla nutrizione enterale tramite Sonda Naso Gastrica (SNG); il trattamento deve essere avviato precocemente non oltre 5-7 giorni nei pazienti normonutriti e non oltre le 24-72 ore nei pazienti malnutriti. Va sottolineato come la presenza del SNG alimentare non escluda il rischio di aspirazione e che non debba superare le 2-3 settimane di permanenza a causa dei rischi connessi come decubiti.(117) Nei soggetti con disfagia persistente per i quali è ipotizzabile una durata superiore a due mesi, è indicato prendere in considerazione il ricorso alla Gastrostomia Endoscopica Percutanea (PEG) entro 30 giorni, da praticarsi non prima di 4 settimane dall'evento. Neppure la PEG garantisce in modo assoluto la protezione dell'aspirazione tracheobronchiale. $(108,117)$ La Nutrizione Enterale verrà sospesa nel momento in cui l'assunzione di alimenti per bocca diverrà attuabile e coprirà il $75 \%$ del fabbisogno nutrizionale.(117)

- Malgrado l'accurata valutazione e attuazione delle misure preventive potrebbe manifestarsi la polmonite da aspirazione. Nello specifico monitorare e riferire al medico tutti i cambiamenti a carico del modello respiratorio di modo da avviare in modo tempestivo una terapia farmacologia specifica. Le cefalosporine di terza generazione sono il trattamento di prima scelta, mentre le penicilline, spesso citate in passato come antibiotico standard per la polmonite $\mathrm{d}$ aspirazione, potrebbe essere inadeguata. L'uso dei corticosteroidi è associato ad un miglioramento del quadro radiografico polmonare. $(108,113)$

Nell'ambito educativo-riabilitativo l'infermiere dovrà coinvolgere attivamente il paziente e i caregivers in tutte le tappe del processo decisionale e nello specifico dovrà:

- Fornire istruzione sulle prescrizioni alimentari: cibi da preferire, variazioni di consistenza (bolo ben compatto e con un buon grado di viscosità) e alimenti da evitare (alimenti a doppia consistenza, filamentosi, alimenti solidi di difficile gestione in bocca, alcolici, pane, crackers e grissini, frutta secca, frutta fresca a chicchi, gelati con pezzi di nocciole, scaglie di cioccolato, canditi).(113,115, 118) Inoltre è fondamentale dare istruzioni sulle modalità di preparazione dell'acqua a consistenza modificata tramite addensante.(118)

- Istruire il paziente e i caregivers sulle misure di sicurezza da mantenere durante la deglutizione, su tutte il mantenimento di una postura diritta a $90^{\circ}$ che deve essere mantenuta per almeno 30 minuti dopo il pasto. $(108,110,113,114)$ 
- Fornire informazioni sull'utilizzo degli ausili adattivi (posate, bicchieri e piatti) e sugli ausili da evitare (cannucce o siringhe poiché abbreviano il tempo di transito aumentando il rischio di aspirazione).(110,113,118,119)

- Educare i caregivers sull'importanza di alcuni comportamenti e nello specifico: favorire l'assunzione del pasto in un ambiente luminoso e privo di distrazioni; evitare di far parlare il paziente durante i pasti; pianificare i pasti nel momento in cui la persona è maggiormente riposata e procedere lentamente rispettando i tempi esecutivi e di attenzione del paziente; verificare lo stato di vigilanza e reattività; garantire l'igiene del cavo orale prima e dopo il pasto; somministrare cibi o liquidi agri, dolci, salati o piccanti prima dei pasti per stimolare la produzione di saliva e mantenere la mucosa orale idratata nei casi di xerostomia manifesta; stimolare l'appetito presentando il pasto con un aspetto invitante evitandolo di mescolarlo.(113, $118,119)$

- Fornire informazioni sui comportamenti da garantire durante la somministrazione del pasto: sedere sullo stesso livello degli occhi del paziente o più basso; introdurre una quantità di cibo pari ad un cucchiaino di caffè oppure $10-15 \mathrm{~mL}$ per volta; far bare al termine della deglutizione; ispezionare periodicamente il cavo orale per osservare la presenza di residui alimentare ed eventualmente rimuoverli $(108,115)$; controllare la posizione del capo; far eseguire un colpo di tosse dopo 1-2 deglutizioni; in caso di tosse riflessa stimolare il paziente a ricondurre l'atto sotto il controllo volontario coordinando la respirazione e le spinte diaframmatiche; insegnare a mettere il cibo dal lato sano della bocca, nei casi di paralisi unilaterale; evitare di toccare i denti o di posizionare il cibo troppo indietro nella bocca. $(113,119)$

- Istruire i caregivers sugli interventi da assicurare al termine della somministrazione del pasto: lasciare il paziente seduto o semiseduto per 30-60 minuti; eseguire l'igiene orale; monitorare la quantità di alimenti assunti, aggiornando il diario alimentare. $(111,114,118)$

- Fornire istruzione sulla corretta metodica di somministrazione dei farmaci, nei casi particolari sulla corretta triturazione del farmaco e fornire un elenco tascabile dei farmaci per cui non è possibile eseguire la frantumazione.(110,113)

- Istruire i caregivers sul riconoscimento dei segni e dei sintomi di disfagia e sul riconoscere gli episodi di presunta aspirazione.(110) Fondamentale è la formazione sul corretto utilizzo dell'aspiratore e sulle manovre di emergenza specifiche come la manovra di Heimlich.(113)

- Spiegare, sulla base di quanto deciso dal medico specialista, il programma di follow-up deciso per l'assistito. Nei pazienti che seguono una dieta con consistenza modificata o una nutrizione enterale, la deglutizione e lo stato nutrizionale andrebbe valutata con cadenza regolari, dopo la prima settimana di riscontro del problema, successivamente ogni 2-3 mesi per il primo anno e successivamente ogni sei mesi, tale cadenza può subire delle modifiche in base alla gravità del problema e alle percentuali di recupero.(113)

\section{Gli squilibri idroelettrolitici}

L'acqua corporea e l'omeostasi degli elettroliti sono una condizione fondamentale per il corretto funzionamento fisiologico. Gli infermieri hanno la chiave per prevenire, individuare precocemente e trattare lo squilibrio idroelettrolitico, così da poter prevenire le complicanze e ridurre i giorni di permanenza in ospedale.(120)

I disturbi del sodio sono i più comuni disturbi degli elettroliti nella medicina clinica (121); l'iposodiemia è riconosciuta come la più comune alterazione elettrolitica e la sua prevalenza negli Stati Uniti è stimata essere $1,72 \%$ sulla popolazione generale.(122)

Studi epidemiologici hanno riscontrato che l'iposodiemia si verifica in circa l' $1-2 \%$ dei pazienti ricoverati,(122) mentre l'ipersodiemia è meno comune.(123)

Per quanto riguarda il potassio si stima che la prevalenza degli squilibri di potassio ematico riguardi il $2-3 \%$ della popolazione generale e la si riscontra in circa il $21 \%$ dei pazienti ricoverati,(124) ma è clinicamente significativa solamente nel $4-5 \%$ di questi pazienti. L'iperpotassiemia invece è spesso causata da farmaci e per questo si può manifestare in pazienti che presentano più comorbilità e può essere riscontrata nell' $8 \%$ dei pazienti ricoverati.(125)

La prevalenza della carenza di magnesio negli ambienti di cura si attesta intorno al 20\%;(126) mentre l'ipofosfatemia è stata riscontrata in oltre il $2 \%$ dei pazienti ricoverati.(127)

La letteratura suggerisce di differenziare l'approfondimento di competenze specifiche a seconda del tipo di disionia cui ci si trova far assistenza:

\section{IPONATREMIA}

- Conoscere la distribuzione del liquido corporeo intra-extracellulare (LIC-LEC) e il concetto di isoipo-iperosmolarità plasmatica.

- Saper definire l'iponatremia.

- Saper definire il grado di iposodiemia in base ai livelli di sodio sierico.

- Segni e sintomi iponatremia, valutando esame obiettivo, stato mentale ed entrate/uscite.

- Saper posizionare un accesso venoso periferico.

- Conoscere come la quantità di sodio muta nelle varie soluzioni:

1. Soluzione fisiologica $0,9 \%: 154 \mathrm{mEq} / \mathrm{L}$.

2. Soluzione ipertonica $3 \%: 513 \mathrm{mEq} / \mathrm{L}$.

3. Soluzione ipertonica 5\%: $860 \mathrm{mEq} / \mathrm{L}$.

4. Soluzione ipotonica $0,45 \%: 77 \mathrm{mEq} / \mathrm{L}$. 
- Saper distinguere le varie forme di iponatremia:

1. Ipertonica

2. Isotonica

3. Ipotonica:

3.1. Ipervolemica

3.2. Isovolemica

3.3. Ipovolemia

- Conoscere le modalità di presentazione clinica dell'iponatremia, in funzione dei valori di sodiemia $(\mathrm{mEq} / \mathrm{L})$ e in funzione della velocità di insorgenza dell'iponatremia.

- Saper distinguere l'iponatremia a insorgenza acuta dalle forme croniche.

- Conoscere le opzioni terapeutiche dell'iponatremia.

- Conoscere le cause di inappropriata secrezione di ADH (SIADH); segni e sintomi, diagnosi, opzioni terapeutiche di SIADH.

- Saper gestire in emergenza l'iponatremia.

- Saper valutare i tempi di infusione e la velocità di correzione.

\section{IPERNATREMIA}

- Saper definire l'ipernatremia.

- Segni e sintomi ipernatremia, valutando stato mentale, peso, entrate/uscite e stato di idratazione.

- Saper distinguere le varie forme di ipernatremia:

- Ipervolemica

- Isovolemica

- Ipovolemia

- Conoscere le principali opzioni terapeutiche dell'ipernatremia.

- Conoscere le modalità di somministrazione delle opzioni terapeutiche dell'ipernatremia in base alla forma (ipervolemica, isovolemica, ipovolemica).

\section{IPOPOTASSIEMIA}

- Conoscere la distribuzione del potassio all'interno dell'organismo.

- Saper definire l'ipopotassiemia.

- Saper definire il grado di ipopotassiemia in base ai livelli di potassio sierico.

- Segni e sintomi ipopotassiemia.

- Conoscere gli alimenti in grado di influenzare la potassiemia (alimenti ricchi di potassio).

- Conoscere le cause di ipopotassiemia.

- Conoscere le principali opzioni terapeutiche dell'ipopotassiemia.

- Conoscere il rapporto tra $\mathrm{pH}$ e potassio.

- Riconoscere i segni ECG-grafici di ipopotassiemia, in funzione ai livelli di kaliemia.

\section{IPERPOTASSIEMIA}

- Saper definire l'iperpotassiemia.

- Saper definire il grado di iperpotassiemia in base ai livelli di potassio sierico.

- Segni e sintomi iperpotassiemia.

- Conoscere le cause di iperpotassiemia.

- Conoscere le principali opzioni terapeutiche dell'iperpotassiemia.
- Conoscere i farmaci che potrebbero indurre iperpotassiemia.

- Riconoscere i segni ECG-grafici di iperpotassiemia, in funzione ai livelli di kaliemia.

IPERMAGNESEMIA

- Saper definire l'ipermagnesemia.

- Segni e sintomi dell'ipermagnesemia.

- Conoscere le cause di ipermagnesemia.

- Conoscere le principali opzioni terapeutiche dell'ipermagnesemia.

- Riconoscere i segni ECG-grafici di ipermagnesemia.

- Conoscere la distribuzione del magnesio all'interno dell'organismo.

IPOMAGNESEMIA

- Saper definire l'ipomagnesemia.

- Segni e sintomi ipomagnesemia.

- Conoscere gli alimenti in grado di influenzare la magnesemia (alimenti ricchi di magnesio).

- Conoscere le cause di ipomagnesemia.

- Conoscere le manifestazioni cardiovascolari e neurologiche dell'ipomagnesemia.

- Conoscere le principali opzioni terapeutiche dell'ipomagnesemia.

\section{IPOFOSFOREMIA}

- Conoscere la distribuzione del fosforo all'interno dell'organismo.

- Saper definire l'ipofosforemia.

- Segni e sintomi dell'ipofosforemia.

- Conoscere le cause di ipofosforemia.

- Conoscere le manifestazioni neurologiche e cardiopolmonari dell'ipofosforemia.

- Conoscere le principali opzioni terapeutiche.

- Gestire correttamente la terapia infusiva (compatibilità tra farmaci) e le possibili complicanze.

\section{IPERFOSFOREMIA}

- Saper definire l'iperfosforemia.

- Segni e sintomi iperfosforemia.

- Conoscere le cause di iperfosforemia.

- Conoscere le principali opzioni terapeutiche.

- Educazione al paziente per una corretta alimentazione.

\section{Le polmoniti}

La polmonite è una patologia che riveste un'elevata importanza sia per le problematiche legate alla sua gestione clinica che per i costi per la collettività. E'una malattia molto diffusa in tutto il mondo, colpisce circa 450 milioni di persone l'anno ed è responsabile di circa 4 milioni di decessi all'anno, cioè del 7\% della mortalità totale mondiale, conquistando così il sesto posto tra le patologie che causano morte.(128,129) Ha un'incidenza maggiore nei paesi sottosviluppati (carenza norme igieniche e profi- 
lassi).(128) Il sesso maschile è più colpito di quello femminile. Le fasce di età più colpite sono: età $<5$ anni ed età $>75$ anni. In Italia i costi complessivi per la cura delle polmoniti sono stati stimati in 500 milioni di euro; più del $90 \%$ dei costi sono stati associati ai ricoveri ospedalieri.(130,131) Le infezioni rappresentano il 42,9\% dei ricoveri in Medicina Interna, di questi ricoveri il $32 \%$ avviene per infezioni polmonari (CAP, HAV, HCAP, VAP, ab ingestis). Il numero dei ricoveri, i giorni di degenza e il tasso di mortalità aumentano nella fascia di età superiore ai 75 anni. Secondo l'ISTAT nel 2012 in Italia si sono verificati 9241 decessi per polmoniti.(130)

La classificazione epidemiologica è la più usata: CAP (community-acquired pneumonia), HAP (hospital-acquired pneumonia), VAP (ventilator-associated pneumonia), $\mathrm{Ab}$ ingetis, $\mathrm{HCAP}$ (health-care acquired pneumonia).(130)

CAP: sono le polmoniti acquisite in comunità. HAP: sono le polmoniti acquisite in ospedale, insorgono dopo 48 ore dal ricovero od entro le 48 ore dalla dimissione. VAP: sono le polmoniti associate all'uso di ventilatori, si manifestano entro 48 ore dall'intubazione endotracheale. HCAP: sono le polmoniti associate all'assistenza sanitaria. Ab Ingestis: I microrganismi patogeni responsabili della polmonite sono batteri, virus, funghi e parassiti. Gli agenti patogeni raggiungono l'apparto respiratorio per inalazione di aerosol (droplet, via aerea) provenienti da tosse e starnuti, l'aspirazione di germi colonizzati nell'orofaringe, contatto diretto (solitamente attraverso le mani) con persone od oggetti contaminati e più raramente per via ematica (postoperatorio, CVC, CV). La diffusione degli agenti patogeni è favorita dalla presenza di alcuni fattori di rischio, intrinseci ed estrinseci dell'ospite, modificabili e non. Alcuni agenti patogeni sono resistenti all'antibioticoterapia; è fondamentale conoscere i fattori di rischio per guidare la terapia antibiotica contro i patogeni multi resistenti (MRSA, ESBL).(130)

La polmonite può insorgere velocemente con segni e sintomi che compaiono nell' arco di 24-48 ore o manifestarsi più lentamente anche in diversi giorni (tipico dei microrganismi resistenti); i sintomi e i segni cambiano a seconda dell'età, della modalità di trasmissione, dell'agente eziologico e dallo stato di salute della persona e sono: tosse, dispnea, temperatura superiore a $38^{\circ} \mathrm{C}$ od ipotermia inferiore a $35,6^{\circ} \mathrm{C}$ soprattutto negli anziani, malessere generalizzato e sintomi meno comuni, quali emottisi, nausea, vomito, stato confusionale, cadute a terra specie nei soggetti anziani. Alla dimissione non è richiesta la risoluzione dei sintomi specifici della malattia, ma, secondo le linee guida NICE del 2014, il paziente non può essere dimesso se nelle ultime 24 ore non presenta almeno $2 \mathrm{o}$ più di questi criteri di stabilità clinica:(132,133) temperatura inferiore a $37,5^{\circ} \mathrm{C}$, frequenza cardiaca $<100$ $\mathrm{b} / \mathrm{m}$, frequenza respiratoria $<24 \mathrm{atti} / \mathrm{min}$, pressione sistolica $>90 \mathrm{mmHg}$, saturazione $>90 \%$ o $^{\mathrm{PaO}_{2}}>60$
$\mathrm{mmHg}$ in aria ambiente, normale stato mentale, capacità di bere e nutrirsi.(130)

\section{I sanguinamenti gastro-intestinali}

Il sanguinamento gastrointestinale è associato significativamente a morbidità e mortalità.

I fattori predisponenti sono fortemente legati allo stile di vita della persona che ne soffre.(134)

Creare una nuova strategia mirata ad educare gli utenti per migliorare la consapevolezza del problema potrebbe aiutare efficacemente a ridurli e a portare il tasso di mortalità quasi allo zero.

Le emorragie digestive rappresentano una evenienza sempre più frequente che impegna pesantemente le strutture sanitarie: negli Stati Uniti si verificano ogni anno 60,6 casi ogni 100.000 abitanti di emorragia digestiva superiore $(134,135)$ e circa 20 casi/100.000 abitanti di emorragia digestiva inferiore. In particolare sono in aumento le emorragie digestive correlate al consumo crescente di farmaci antinfiammatori non steroidei (FANS) e di antiaggreganti piastrinici, soprattutto in età avanzata. Non vi sono ancora dati certi sulla prevalenza di sanguinaamenti gastroenterici in persone che utilizzano i nuovi anticoagulanti orali (NAO).

La mortalità per emorragia digestiva superiore è ancora particolarmente elevata nei soggetti anziani con comorbidità, raggiungendo anche il $10-14 \%$ ed il sanguinamento da varici esofagee comporta una mortalità di circa il 20\% entro 6 settimane dall'evento emorragico.(135) Si definisce un'emorragia digestiva il sanguinamento attivo o recente la cui origine è situata nell'apparato digerente (tubo digerente principalmente e più raramente nelle vie biliari e pancreatiche).(136)

I sanguinamenti si distinguono in emorragia digestiva superiore, che interessa il tratto gastrointestinale fino al legamento di Treitz ed emorragia digestiva inferiore che ha origine al di sotto dello stesso.

Si distinguono 3 tipi di sanguinamento:

- visibile, si manifesta come sangue rosso vivo o nerastro nel vomito o nelle feci;

- occulto, confermato tramite test del sangue occulto fecale positivo con associata o meno anemia sideropenica;

- oscuro, qualsiasi sanguinamento visibile od occulto, intermittente o persistente, di qualsiasi tratto del tubo digerente la cui causa risulta sconosciuta anche dopo esame endoscopico o valutazione radiologica.(137)

Le cause più comuni di emorragia digestiva superiore sono:

- ulcera peptica $(35-50 \%)$

- erosione gastroduodenale $(8-15 \%)$

- esofagite $(5-15 \%)$

- varici esofagee $(5-10 \%)$

- Mallory-Weiss (15\%) 
- $\quad$ neoplasie (1\%)

- malformazioni vascolari (5\%) rare (5\%).(138)

Le cause più comuni di emorragia digestiva inferiore sono:

- diverticoli (20-65\%)

- coliti ischemiche (1-19\%)

- angioectasie (3-15\%)

- emorroidi (2-10\%)

- neoplasie colon rettale (17\%)

- $\quad$ sanguinamento post polipectomia (2-8\%)

- malattie infiammatorie intestinali: morbo di Crohn $(1,2-6 \%)$, coliti ulcerose $(0,1-4,2 \%) .(139)$

L'uso di aspirina e/o FANS sembra aumentare il rischio di emorragie intestinali.(140)

I sanguinamenti gastro-intestinali si possono presentare in varie forme:

- Ematemesi se il sangue si presenta con il vomito (rosso vivo se recente, caffeano se ha ristagnato nello stomaco).

- Melena, feci picee, semiliquide, maleodoranti (emoglobina degradata ad ematina). Per produrre un'evacuazione di feci nere sono necessari circa $100 \mathrm{ml}$ di sangue.

- Ematochezie o rettorragia, emissione di sangue rosso vivo dal retto.

- Sangue occulto se rilevabile solo con misurazione chimica all'esame delle feci.

- Talora solo segni clinici di sanguinamento, quali anemia, ipotensione ortostatica, dispnea, shock.

Il primo passo nella diagnosi della causa del sanguinamento è quello di differenziarlo tra superiore ed inferiore.

Fondamentale sono l'anamnesi e l'esame obiettivo del paziente.

Le indagini diagnostiche utilizzate sono:

- endoscopia del tratto digestivo superiore e/o inferiore per una diretta visualizzazione della sede di sanguinamento e per l'eventuale trattamento della lesione;

- angiografia per evidenziare la sede del sanguinamento o la presenza di vascolarizzazione anomala, può consentire l'embolizzazione dei vasi sanguinanti;

- scintigrafia con radioisotopi che può identificare la presenza di emorragie anche minime;

- endoscopia con videocapsula;

- TAC;

- RM-enteroscopia.(141)

Nei pazienti con emorragia digestiva acuta è raccomandata la valutazione immediata dello stato emodinamico ed il tempestivo ripristino del volume intravascolare usando cristalloidi nel caso vi sia instabilità emodinamica (raccomandazione basata sull'esperienza) al fine di ridurre la mortalità.

Nel paziente emorragico si raccomanda un livello di emoglobina tra 7 e $9 \mathrm{~g} / \mathrm{dL}$; livelli maggiori sono necessari nei pazienti con comorbilità significative, ad esempio cardiopatia ischemica (raccomandazione basata sull'esperienza).(142)
É fondamentale valutare attentamente lo stato emodinamico del paziente emorragico provvedendo innanzitutto alla sua stabilizzazione, qualora fossero presenti segni di shock, per poter eseguire l'esame endoscopico che rappresenta l'elemento diagnosticoterapeutico cardine per la gestione di questi pazienti.

Compito dell'infermiere è riconoscere il tipo di sanguinamento e valutarne l'entità.

In urgenza attuare i protocolli vigenti o in loro assenza allertare il personale medico, recuperare il carrello delle urgenze, somministrare $\mathrm{O}_{2}$ terapia con maschera ad alti flussi (15 L/min), posizionare uno o più accessi periferici di grosso calibro ed iniziare ad infondere soluzione fisiologica, eseguire prelievo venoso per emocromo, coagulazione e gruppo.

Assistere nel processo di emotrasfusione, nel posizionare la sonda di Blakemore che assicura un tamponamento locale nei pazienti con sanguinamento da varici esofagee.(143)

Nella gestione del paziente con sanguinamento gastrointestinale sono state individuate diverse conoscenze (sapere), abilità e capacità tecniche (saper fare) e qualità relazionali (saper essere) che l'infermiere deve possedere.

All'interno delle conoscenze da possedere è indispensabile:

- Conoscere dove è collocato il carrello dell'urgenza e dove sono i farmaci da frigo.

- Conoscere gli aspetti eziologici e fisiopatologici del sanguinamento GI.

- Conoscere gli accertamenti diagnostici per lo studio dei sanguinamenti.

- Allertare centro trasfusionale.

- Assegnazione codice triage (in DEA).

- Gestire richiesta e somministrazione di emazie.

- Esecuzione delle prove crociate.

- Rilevazione dei bisogni assistenziali e pianificazione dell'assistenza.

- Pianificare la dimissione favorendo la continuità assistenziale.

- Registrazione degli eventi clinici ed assistenziali da trasmettere ai turni successivi.

- Riconoscere i segni e sintomi di un progressivo deterioramento del quadro clinico.

- Eventualmente saper allertare il chirurgo ed il rianimatore.

- Coordinare/partecipare alla stesura di linee guida e percorsi assistenziali per pz con sanguinamenti GI.

- Valutare il rischio post endoscopia con lo Score di Rockall.(142)

Per ciò che riguarda abilità e capacità tecniche, la letteratura suggerisce come fondamentali:(135-143)

- Misurare i parametri vitali e impostare un monitoraggio a seconda del rischio di deterioramento clinico.

- Posizionare un accesso vascolare stabile di calibro adeguato (il minore possibile, considerando la portata dello stesso e le necessità di infusione) e assicurarne il mantenimento. 
- Se indicato, posizionare un catetere vescicale.

- Saper gestire la terapia endovenosa specifica.

- Far rimuovere e conservare occhiali e protesi dentarie prima dell'esecuzione dell'esame endoscopico.

- Posizionare quando indicato un sondino nasogastrico.

- Monitorare e descrivere quantità e qualità delle scariche.

- Posizionare la sonda di Blakemore.

\section{La sepsi}

La sepsi, assieme allo shock settico, rappresenta uno dei più importanti problemi sanitari $(144,145)$ che colpisce ogni anno milioni di persone nel mondo (144) e che, nel 2013 negli Stati Uniti, ha comportato una spesa sanitaria di 23,7 miliardi di dollari.(146) Essa può svilupparsi da molteplici infezioni e spesso si tratta di una sindrome difficile da diagnosticare (146) in quanto i segni e i sintomi possono essere lievi e facilmente trascurabili cosicché la condizione clinica può rapidamente peggiorare fino allo shock settico.(147)

La reale incidenza è sconosciuta, tuttavia i report epidemiologici ne indicano un incremento probabilmente correlato all'invecchiamento generale della popolazione ed alle maggiori comorbidità che ne conseguono e ad un maggiore riconoscimento della condizione clinica.(144) Si tratta di una sindrome associata ad una elevata morbilità e mortalità $(25 \%)$ : (146) è considerata tra le cause principali di decesso e di condizioni di salute critiche.(144)

Negli ultimi anni si è notato un aumento della presa in carico dei pazienti con sepsi all'interno dei reparti medici e chirurgici e, in diversi paesi del Nord America e dell'Europa, è segnalato che il 14$80 \%$ dei pazienti ricoverati in tali reparti sviluppa sepsi.(148)

Come per l'infarto miocardico acuto, lo stroke e il politrauma, anche per la sepsi sono stati riscontrati outcomes migliori quando è presente l'identificazione precoce e una gestione appropriata del quadro settico nelle ore iniziali,(145) e l'infermiere svolge un ruolo centrale ed attivo durante tutto il percorso di cura. Trattandosi di una sindrome difficile da diagnosticare l'identificazione precoce è fondamentale per la corretta gestione del quadro settico; affinché ciò avvenga è necessario che l'infermiere abbia competenze avanzate non solo per la valutazione delle condizioni cliniche, che può avvenire anche attraverso l'uso discale di valutazione, ma soprattutto per la valutazione del rischio di sviluppare la sepsi. Nella fase acuta la conoscenza approfondita delle linee guida e dei bundles, delle terapie da somministrare (comprese interazioni farmacologiche ed effetti collaterali) e dei devices utilizzati ne permettono la gestione ottimale. La prevenzione dovrebbe essere il punto cardine della lotta alla sepsi; infermieri con competenze avanzate dovrebbero creare e divulgare programmi per la prevenzione delle ICA, per la prevenzione delle infezioni comunitarie, per la corretta igiene ambientale, per i corretti comportamenti da adottare in comunità $\mathrm{e}$ in ambienti ospedalieri.

\section{Il monitoraggio cardiaco}

L'elettrocardiogramma (ECG) standard rappresenta la principale e più comune metodica d'indagine cardiologica: le forme d'onda che ne derivano rappresentano l'attivazione elettrica specifica delle varie camere cardiache. I meccanismi alla base dei segnali elettrici cardiaci possono essere ricondotti ai processi di ripolarizzazione, diffusione e ripolarizzazione delle membrane cellulari del miocardio.(149)

Presupposto essenziale per una corretta analisi del segnale ECG è avere a disposizione una buona traccia.

L'ECG a riposo fotografa l'attività cardiaca nel momento in cui viene effettuato, mentre il monitoraggio ECG è una registrazione continua dell'attività elettrica del cuore. Il segnale ECG viene oggi derivato da una moltitudine di strumenti che comprendono: elettrocardiografi, sistemi di registrazione Holter, monitor posto-letto, telemetrie e sistemi di ergometria.

La tecnica di monitoraggio ECG più completa è quella a 12 derivazioni ed anche se non viene utilizzata per il monitoraggio continuo, è importante conoscerne gli aspetti basilari. Per la registrazione occorrono 10 elettrodi: uno su ciascun polso, uno per caviglia e sei precordiali. La tipologia di cavi è essenzialmente di due tipi: un tipo misura la differenza tra elettrodo positivo e negativo. La sectipologia è un cavo unipolare che misura la variazione di potenziale di un singolo elettrodo: aVR, aVL, aVF e da V1 a V6 sono i nove unipolari. Le derivazioni unipolari del torace (derivazioni precordiali) si ottengono collegando l'elettrodo esplorante in punti ben definiti del torace: V1 IV spazio intercostale sulla margino-sternale $\mathrm{dx}$ V2 IV spazio intercostale sulla margino-sternale sx V3 punto di mezzo tra V2 e V4 V4 V spazio intercostale sulla emiclaveare V5 V spazio intercostale sulla ascellare anteriore V6 V spazio intercostale sulla ascellare media.

E' importante sottolineare che qualunque modificazione nel posizionamento degli elettrodi provoca un'alterazione delle onde.

Il monitoraggio ECG continuo permette la visualizzazione costante dell'attività cardiaca. Si effettua mediante l'utilizzo di monitor collegati alla persona attraverso elettrodi posti sul torace. Solitamente vengono utilizzati tre elettrodi da posizionare privilegiando le prominenze ossee al fine di prevenire al massimo artefatti da movimento del paziente: l'elettrodo rosso deve essere posto al di sotto della clavicola $\mathrm{dx}$, sulla linea medio claveare, L'elettrodo giallo deve essere posto al di sotto della clavicola sx, sulla linea medio - claveare, l'elettrodo verde deve essere posizionato tra il $\mathrm{VI}^{\circ}$ e il $\mathrm{VII}^{\circ}$ spazio intercostale, sulla linea medio claveare sx.

L'obiettivo di questo tipo di monitoraggio è quello 
di rilevare la frequenza cardiaca ed il ritmo cardiaco; non è idoneo per identificare aritmie sofisticate.(150)

Secondo l'American College of Cardiology Emergency Cardiac Care Committee i pazienti vengono suddivisi in tre diverse classi sulla base della forza dell'indicazione al monitoraggio continuo ECG:

Classe 1: il monitoraggio è indicato nella maggior parte (se non in tutti i pazienti) appartenenti a questa classe.

Classe 2: il monitoraggio può essere indicato per alcuni pazienti ma non deve essere considerato necessario per tutti.

Classe 3: comprende pazienti a basso rischio per cui il monitoraggio non serve.(149)

\section{Monitoraggio cardiaco nell'ischemia}

\section{Classe 1}

Comprende tutti quei pazienti a rischio significativo a rischio di aritmie improvvise pericolose per la vita, ovvero:

- pazienti rianimati dopo arresto cardio-circolatorio (ACC)

- pazienti in fase iniziale di sindrome coronarica acuta: con slivellamento o meno del tratto ST e angina instabile

- pazienti sottoposti a intervento cardiochirurgico (bypass coronarico, sostituzione valvolare)

- pazienti sottoposti a impianto di Pacemaker (PMK) o defibrillatore automatico impiantabile (ICD) con assenza spontanea del ritmo o instabilità emodinamica e pazienti sottoposti a impianto PMK temporaneo

- paziente con blocco atrio ventricolare (BAV) (pazienti con Mobitz II avanzato (2:1 o superiore), BAV di secondo grado o blocco completo)

- pazienti con QT allungato associato ad aritmie ventricolari

- pazienti con insufficienza cardiaca acuta e edema polmonare

- pazienti con indicazione a cure intensive. Il monitoraggio EGC è indicato in tutti i pazienti con trauma maggiore, insufficienza respiratoria acuta, sepsi, shock, embolia polmonare, chirurgia maggiore (soprattutto se anziani con storia di malattia coronarica, insufficienza renale con alterazioni elettrolitiche)

- pazienti sottoposti a procedure diagnostiche in anestesia o sedazione profonda: dovrebbero essere monitorati fino al loro risveglio o fino a stabilità emodinamica

- pazienti con aritmie ed emodinamicamente instabili. Le aritmie sono considerate benigne se non c'è alla base una patologia cardiaca; ma possono essere letali in presenza di malattie cardiache importanti.

Classe 2

Per i pazienti appartenenti a questa categoria il monitoraggio può essere indicato in alcuni casi specifici:
- pazienti con IMA in fase post acuta. Nella maggior parte dei casi le aritmie ventricolari si verificano nelle prime 24 ore. I fattori predittivi di aritmie ventricolari tardive sono: ipertensione, broncopatia cronico-ostruttiva (BPCO), precedenti IMA, le variazioni del tratto $\mathrm{ST}$ in fase acuta e pressione arteriosa sistolica ridotta

- pazienti con dolore toracico. Il dolore toracio è una delle cause più frequenti di accesso al Pronto Soccorso e richiede un'attenta valutazione diagnostica a causa delle sue conseguenze potenzialmente fatali. Un approccio più razionale per decidere se iniziare un monitoraggio ECG continuo è quello di utilizzare uno strumento di previsione di rischio nel momento in cui il paziente si presenta in Pronto Soccorso (PS) con dolore toracico. Il rischio Goldman identifica i pazienti in rischio alto, moderato, basso o molto basso basato sul tracciato ECG, sull'anamnesi e sull'esame obiettivo. La previsione di rischio viene fatta sulla base di cinque variabili: i) Sospetto di IMA dall'ECG iniziale con sopra slivellamento di ST uguale $\geq 1 \mathrm{~mm}$ o onde $\mathrm{Q}$ patologiche in almeno due derivazioni; ii) Sospetta ischemia nell'ECG iniziale con sotto slivellamento del segmento ST di $\geq 1 \mathrm{~mm}$ o inversione dell'onda $\mathrm{T}$ in almeno due derivazioni; iii) $\mathrm{PaO}$ sistolica $<110$; iv) Rantoli bilaterali; v) Storia di angina instabile. In questi pazienti il monitoraggio dovrebbe essere proseguito per 12-24 ore fino a quando l'IMA viene escluso dai marcatori negativi (troponina)

- pazienti in trattamento con farmaci anti aritmici.

- pazienti sottoposti ad impianto di PMK non dipendenti da stimolazione. In questo caso l'obiettivo del monitoraggio non è di rilevare bradicardie pericolose per la vita ma confermare la presenza dello stimolo da parte del device e la programmazione appropriata.

- pazienti in studio per sincope fino a quando la causa aritmica viene esclusa.

Classe 3

Appartengono a questa classe pazienti post operati a basso rischio di aritmie (soggetti giovani, soggetti in assenza di malattie cardiache), pazienti con FA cronica, pazienti sottoposti a dialisi.(149)

\section{Monitoraggio del tratto ST nell'ischemia}

Classe 1

- pazienti con sindrome coronarica acuta (SCA) in fase iniziale.

- paziente con dolore toracico e sintomi dolorosi equivalenti

- pazienti sottoposti ad angiografia risolutiva

- pazienti con angina. Gli obiettivi del monitoraggio è confermare la diagnosi dell'evento ischemico transitorio, rilevare eventuali aritmie maligne durante lo spasmo coronarico, valutare l'efficacia del trattamento.

Classe 2

- pazienti con IMA post acuto. Il monitoraggio non 
deve essere interrotto nei pazienti che hanno avuto dolore toracico ricorrente e ri elevazione degli enzimi cardiaci.

- pazienti ad alto rischio dopo chirurgia maggiore.

\section{Monitoraggio dell'intervallo QT e monitoraggio ECG}

Esistono diverse situazioni cliniche associate a modificazioni del tratto QT che possono rappresentare un rischio aumentato di sincope, torsione di punta e morte improvvisa. Tra queste le più frequenti sono: sovradosaggio di farmaci che possono aumentare il tempo di ripolarizzazione ventricolare, ischemia e infarto, alterazioni elettrolitiche, diminuzione improvvisa della frequenza cardiaca ed eventi neurologici acuti.

Il monitoraggio dell'intervallo QT ha maggiore priorità se il paziente presenta fattori di rischio per torsione di punta: età avanzata, malattie cardiache, alterazione degli elettroliti, malnutrizione, predisposizione genetica, disordini metabolici. Inoltre pazienti con QT allungato sono a rischio immediato di torsione di punta se presentano aritmie correlate tra cui bradicardie improvvise con pause importanti.(149)

\section{La ventilazione non-invasiva}

Il concetto di ventilazione meccanica non invasiva (NIV) si riferisce alla capacità di fornire un supporto ventilatorio attraverso le vie aeree superiori del paziente, utilizzando maschere o altri devices. La tecnica si distingue da quelle che bypassano le vie aeree, attraverso il posizionamento di un tubo tracheale, maschera laringea, o tracheotomia e che pertanto, sono considerate invasive.

Le modalità principali di trattamento NIV sono:

- la pressione positiva continua delle vie aeree (continuous positive airway pressure, CPAP), che prevede, in respiro spontaneo, una pressione positiva applicata per l'intero ciclo respiratorio attraverso l'interfaccia, senza fornire un supporto pressorio alla ventilazione;

- la pressione positiva bifasica delle vie aeree (bilevel positive airway pressure, BiPAP), che prevede l'impostazione di un supporto pressorio (pressione positiva inspiratoria delle vie aeree, inspiratory positive airway pressure, iPAP) che, quando il paziente inizia l'atto inspiratorio, va a sommarsi ad un livello di pressione positiva espiratoria delle vie aeree (expiratory positive airway pressure, ePAP). La BiPAP viene anche detta ventilazione non invasiva a pressione positiva.(151)

In base alle Linee guida ed alla letteratura internazionale, le principali indicazioni all'utilizzo della NIV sono: (152)

- BPCO: il paziente presenta, di base, un aumento del lavoro respiratorio, sia in inspirazione che in espirazione, correlato all'aumento delle resistenze al flusso aereo e determina un aumento del lavoro respiratorio in inspirazione $\left(\mathrm{pH}<7,35-\mathrm{pCO}_{2}>6,5\right.$ (65
$\mathrm{mmHg}$ ) - FR >23), parametri che persistono dopo terapia con broncodilatatori ed ossigenoterapia;

- Edema polmonare acuto: l'edema polmonare acuto cardiogeno (EPAC), è tra le cause più frequenti di insufficienza respiratoria acuta nei reparti di medicina interna. E' una forma di lung failure (deficit degli scambi gassosi), caratterizzata da ipossiemia, con pressione parziale di anidride carbonica $(\mathrm{pCO} 2)$ di solito normale, a differenza dalle forme di pump failure (insufficienza della pompa ventilatoria).

Le controindicazioni assolute riguardano, severe deformità facciali, ustioni facciali ed ostruzioni delle vie aeree; quelle relative riguardano $\mathrm{pH}<7,15(\mathrm{pH}<7,25$ in aggiunta ad altri fattori), GCS $<8$, confusione/agitazione e problemi cognitivi (garantire un maggior monitoraggio). Il coma è considerato una controindicazione assoluta per la perdita di protezione delle vie aeree, anche se uno studio ha riportato risultati positivi in pazienti con GCS $<8$ con un maggior monitoraggio. Analogamente, confusione, agitazione e alterazione dello stato cognitivo rendono l'applicazione della NIV più difficile ma non si dovrebbe escludere il suo utilizzo.(152)

L'utilizzo della NIV provoca minor compromissione dello stato emodinamico, per questo l'ipotensione non dovrebbe escluderla a favore della intubazione. Mentre, un'aritmia significativa che provoca ipotensione porta alla scelta dell'intubazione.

La pianificazione è un momento fondamentale nell'applicazione della NIV, devono essere disponibili ventilatore e tutti i presidi necessari, tubo, raccordo, filtro, maschere di vario tipo e sistemi di fissaggio. Il circuito deve essere testato prima dell'utilizzo.

Il personale medico ed infermieristico rivestono un ruolo cruciale nel favorire la compliance del paziente. L'approccio iniziale dovrebbe consistere nell'illustrare al paziente le varie componenti del sistema e nel trovare l'interfaccia più adatta al paziente; il paziente deve essere istruito a sincronizzare adeguatamente i suoi atti respiratori con il respiratore ed incoraggiato a comunicare ogni possibile disagio o paura.(153)

L'assistenza: le principali diagnosi infermieristiche per il paziente in trattamento ventilatorio sono:

1. alterazione della respirazione correlata all'utilizzo di un dispositivo esterno per la respirazione

2. alterazione degli scambi gassosi correlati alla perdita di tessuto polmonare per intrappolamento d'aria negli alveoli e ventilazione inadeguata (perfusione)

3. perdita tissutale compromessa dalla bassa saturazione d'ossigeno

4. nutrizione inadeguata per il fabbisogno necessario alle esigenze dell'organismo correlata alle condizioni della malattia ed alla terapia con NIV

5. ansia e fatica legata all'ipossia ed all'utilizzo della maschera

6. paura (claustrofobia) legata alla fame d'aria ed alla ventilazione meccanica

7. compromissione della comunicazione verbale correlato all'utilizzo della maschera 
8. compromissione dell'integrità cutanea (ulcere cutanee), a livello del naso correlato all'uso di maschere rigide

9. alterazione della mucosa orale correlata al flusso di ossigeno continuo

10. intolleranza di attività legata all'insufficienza respiratoria ed all'utilizzo del ventilatore

11. rischio di infezione correlato alle secrezioni abbondanti per la riacutizzazione

12. complicazioni legate all'utilizzo della NIV a lungo termine o alle complicanze correlata alla malattia acuta.(154)

Le interfacce disponibili sul mercato sono: boccagli (includono solo la bocca); maschere nasali (includono il naso ma non la bocca); olive nasali (includono solo le narici); maschere oro-nasali (includono il naso e la bocca); maschere oronasali totali o total full face mask (includono tutto il volto); caschi (includono tutto il capo incluso il collo).

Le caratteristiche di un'interfaccia ideale sono buona tenuta, atraumatica, leggera, di lunga durata, indeformabile, anallergica, bassa resistenza al flusso d'aria, basso costo e disponibilità in varie misure. Nella scelta dell'interfaccia vanno considerate le preferenze del paziente ed il suo comfort che, assieme all'interazione paziente-ventilatore, sono i principali determinanti del successo o del fallimento della NIV. Il paziente deve essere istruito sul sistema di fissaggio e sul sistema di sicurezza antisoffocamento.(155)

Il monitoraggio durante la NIV è molto importante e prevede:

- saturazione dell'ossigeno monitorata costantemente con pulsossimetro. Nelle prime ore devono essere monitorati i parametri, per valutare l'effetto emodinamico della procedura. La saturazione dell'ossigeno deve essere mantenuta $>90 \%$ (154)

- l'emogas arterioso deve essere eseguito entro 15 min dall'inizio della NIV per valutare la risposta e quindi ad intervalli prestabiliti (30 min, 1 h, 3 h, 6 h);(151) se necessario, devono essere variate le impostazioni del ventilatore

- gli infermieri che si prendono cura di questi pazienti devono essere clinicamente competenti in quanto richiedono una valutazione ed un'osservazione stretta degli effetti del trattamento della NIV - il monitoraggio dell'ECG è consigliato se il paziente ha una $\mathrm{FC}>120 \mathrm{bpm}$ o se è presente un'aritmia o una cardiomiopatia.(152)

La complicanza maggiore della NIV è data dalle lesioni cutanee della piramide nasale che possono essere ridotte effettuando una rigorosa cura della cute, utilizzando maschere pulite ed effettuando la rotazione delle interfacce con modelli diversi durante la NIV.

Altre complicanze sono distensione gastrointestinale, singhiozzo, inalazione da rigurgito gastrico o vomito. Per evitare la distensione gastrica, eventuale vomito ed inalazione è consigliabile posizionare un sondino naso-gastrico e quando possibile ridurre le pressioni del ventilatore.
Le perdite aeree sono un'ulteriore complicanza che possono essere evitate dall'utilizzo di interfacce di misura adeguata al volto del paziente.(155) Altre complicanze possono essere pneumotorace, ipotensione, aritmia, distress respiratorio progressivo, sanguinamento gastrointestinale, asfissia, congiuntivite, sinusite, irritazione degli occhi, ictus, compromissione neurologica (coma) e arresto cardiorespiratorio.(155)

La sedazione può essere effettuata durante il trattamento NIV per migliorare la compliance del paziente. Le molecole da utilizzare, sulla base delle loro proprietà, sono principalmente dexmedetomidina, remifentanil e ketamina che presentano il miglior profilo di efficacia e sicurezza, propofol e morfina occupano una posizione intermedia, mentre le benzodiazepine andrebbero per lo più evitate.(153) La sedazione può essere potenzialmente molto pericolosa, ed è pertanto necessario uno stretto monitoraggio del paziente mediante emogasanalisi seriati, valutazione dei parametri cardio-polmonari e livello di sedazione. A tal proposito l'utilizzo di una scala di sedazione come la Ramsey Sedation Scale somministrata al paziente ogni 60 min, può essere utile per oggettivare il livello di coscienza e cogliere precocemente segni di deterioramento clinico.(153)

\section{L'ecografia operativa bedside}

Ad oggi la definizione linguistica di ecografia è: Tecnica diagnostica basata sull'eco di onde ultrasoniche ad alta frequenza inviate sull'organo in esame, (156) ma l'ecografo non è da considerare solo come strumento diagnostico, ma anche soprattutto come supporto a procedure tecniche. Questo fa sì che si possa considerare uno strumento multidisciplinare e ne permette l'utilizzo in più contesti.

A livello giuridico non si rintracciano norme che disciplinano l'utilizzo dell'ecografo come strumento ad uso esclusivo da parte di specialisti in radiologia, la figura dell'infermiere si inserisce in questo contesto tecnico, grazie anche ad uno sviluppo professionale e legislativo. A seguito della L. 42/99 Disposizioni in materia di professioni sanitarie, con l'abolizione del mansionario e il riconoscimento dell'autonomia professionale, le competenze dell'infermiere sono definite attraverso: Il profilo professionale (D.M. 739/94); Il codice deontologico del 2009; nello specifico l'articolo 11 sottolinea che l'infermiera fonda il proprio operato su conoscenze validate ed aggiorna saperi e competenze attraverso la formazione permanente, la riflessione critica sull'esperienza e la ricerca e nell'articolo 12 che ribadisce che l'infermiere riconosce il valore della ricerca della sperimentazione clinica e assistenziale per l'evoluzione delle conoscenze e per $i$ benefici sull'assistito; l'ordinamento didattico; il quadro normativo. La normativa ha avuto altre evoluzioni e nella Conferenza Stato-Regioni del 13 novembre 2014 si evince che lo sviluppo specialistico della professione sia stato riconosciuto come non più rin- 
viabile, e questo trova spazio d'applicazione nel comma 566 della Legge di stabilità del governo Renzi dove si ribadisce che l'infermiere sia responsabile del processo assistenziale prevedendo l'acquisizione di competenze avanzate e specialistiche al passo con l'evoluzione tecnico-scientifica (Legge 23 dicembre 2014, n 190, Gazzetta ufficiale del 29 dicembre).(157)

L'utilizzo degli ultrasuoni da parte del personale infermieristico è da intendere come un supporto alle tecniche assistenziali, è dunque da considerarsi un'ecografia operativa e non diagnostica. Con specifici percorsi formativi è possibile pensarne l'utilizzo in ogni contesto lavorativo dove vi sia a disposizione la strumentazione. Date le sue peculiarità trova impiego e sviluppo sia nelle aree primarie che quelle di emergenza, terapia intensiva, area medica, chirurgica e salute mentale. Gli utilizzi più frequenti lo vedono implicato per il supporto a tecniche infermieristiche quali: il posizionamento di accessi venosi ecoguidati; nella valutazione del livello di instabilità attraverso la valutazione della vena porta e della pleura in pazienti scompensati piuttosto che del pattern polmonare per indagare condizioni definite di polmone wet; per valutare il corretto posizionamento del catetere vescicale attraverso la visualizzazione del palloncino in vescica; per valutare la quantità di ristagno vescicale pos-minzionale o per la valutazione della loggia prostatica per evidenziare probabili difficolta d'inserzione del catetere prima di effettuare la manovra; gli ultrasuoni possono essere di supporto per il posizionamento del sondino naso gastrico; evidenziare stasi intestinali, presenza di fecalomi; troviamo anche l'uso degli ultrasuoni da parte del personale infermieristico nell'ambito ginecologico come followup nel primo trimestre di gravidanza o per tecniche di procreazione assistita, dove l'infermiere ha finalità di monitoraggio; assistenza durante esecuzione di paracentesi evacuativa ed esplorativa; altro aspetto è l'uso delle immagini ecografiche come supporto al personale infermieristico addetto al triage per attribuire adeguato codice di priorità assistenziale e per la rivalutazione dei pazienti critici durante il periodo di attesa della visita specialistica.

Lo studio AWHONN, 2010 (158) non limita l'uso dell'ecografo da parte degli infermieri, ma suggerisce una formazione didattica specifica al fine di ottenere scansioni valide ed efficaci. $(158,159)$ La ricerca ha dimostrato che vi è anche un aspetto economico favorevole ad investire risorse per formare personale infermieristico ad usare l'ecografo.(159)L'uso dell'ecografo trova anche ruolo fondamentale nel monitoraggio del followup inerente ai pazienti con scompenso cardiaco, ma sono necessari ulteriori studi su larga scala per definire meglio i vantaggi (160) come anche il monitoraggio infermieristico durante il primo trimestre di gravidanza.(159) Al fine di valutare il corretto posizionamento del sondino naso gastrico è possibile avvalersi degli ultrasuoni, ma la revisione sistematica trovata evidenzia troppa disparità tra gli studi analizzati e suggerisce la necessità di effettuare nuovi studi specifici per determinare se l'uso dell'ecografo possa sostituire la diagnostica effettuata tramite radiografia per accertare il corretto posizionamento del sondino naso gastrico.(161) Gli studi scientifici analizzati al fine della ricerca fanno riferimento all'uso dell'ecografo finalizzato alla raccolta di urine nei pazienti pediatrici. E'auspicabile riportare anche nell'uso quotidiano questa tecnica per i pazienti adulti, soprattutto dove, vi siano problemi neurologici o comunque per visualizzare la loggia prostatica prima di effettuare la manovra evidenziando con anticipo probabili difficoltà che potrebbero compromettere la manovra di cateterizzazione.

Sono numerosi i vantaggi dell'inserimento degli ultrasuoni nella pratica infermieristica. Uno dei più facili da rilevare è riferito all'uso dell'ecografo per la cateterizzazione vescicale dove studi specifici inerenti affermano che questa procedura è responsabile della riduzione delle infezioni delle e procedure non efficaci.(162) Le caratteristiche intrinseche dello strumento permettono la possibilità di scansioni infinite non esponendo a rischi il paziente in quanto non vengono utilizzate radiazioni ionizzanti ed è di facile esecuzione. E' possibile eseguirla anche al letto del paziente.(163) E'applicabile a tutte le persone comprendendo tutte le fasce d'età e non vi sono controindicazioni all'esecuzione. Si deduce dunque che l'uso delle scansioni ecografiche comportano minor complicanze nelle procedure ed in caso di necessità è possibile ripetere l'esame in quanto non invasivo ed economico. Nonostante il costo dell'ecografo rimanga abbastanza elevato è comunque di facile reperibilità, la strumentazione si trova in quasi tutte le unità operative. L'esecuzione dell'esame ecografico è veloce $\mathrm{e}$ se effettuata da personale debitamente formato risulta accurata e completa, apportando allo studio medico diagnostico molte informazioni che possono indirizzare e arricchire il processo diagnostico accelerandone l'operatività curativa, terapeutica ed interventistica. Altra caratteristica apprezzabile ed utilissima è quella che l'esame riproduce immagini in tempo reale, dando all'operatore la possibilità di monitorare evoluzioni cliniche rapidamente.

Nonostante l'ampio sviluppo di questa tecnica vi sono limiti che ne marginano la diffusione. La tecnologia si è evoluta fino a rendere tascabili gli strumenti di dimensioni minori, ma rimangono ancora elevati i costi e questo fa si che non si possano avere a disposizione del personale infermieristico un numero sufficiente di ecografi. È definito che il costo dell'ecografo viene ammortizzato in tre anni quando si sostituisce la tecnica di cateterizzazione vescicale classica con il cateterismo effettuato dopo scansione ecografica che ne determina preventivamente le dimensioni della vescica e della prostata. Questo è responsabile di una riduzione delle infezioni e procedure non efficaci.(162) Ricordiamo in questo contesto che la finalità dell'utilizzo da parte del personale infermieristico vuol essere 
di supporto a procedure assistenziali, uso tecnico valutativo e non strumento diagnostico. Per poter sfruttare l'ecografo nello svolgimento delle procedure infermieristiche è necessaria formazione specifica per ogni singola scansione che si vuole ottenere e spesso anche training specifici per ogni singolo ecografo quando cambia la ditta, questo fa si che il processo educativo e formativo degli operatori che si approcciano a questa tecnica sia lento e necessiti di formazione continua. Oltre che al costo elevato della strumentazione restano elevati anche quelli dei manichini per l'esercitazione in fase formativa d'apprendimento per l'incannulamento dei vasi venosi.(164) Va ricordato inoltre che oltre alla teoria per l'uso dell'ecografo, è indispensabile una buona manualità per ottenere valide scansione, va da sé che l'uso ripetuto nella pratica giornaliera permetta all'operatore di fruirne in modo più appropriato essendo rapido nelle procedure.

L'insegnamento dell'utilizzo dell'ecografo come supporto tecnologico attualmente non rientra in molti ordinamenti didattici dei corsi di laurea in infermieristica. Negli ultimi anni sono stati attivati master di primo livello post laurea della durata di un anno, corsi di approfondimento o di alta formazione per affinare le tecniche di impianto di PICC e Midline. Dalla letteratura analizzata ne deriva una disomogeneità rispetto alla formazione richiesta per poter effettuare monitoraggi ecografici in autonomia e poter usare questa tecnica per il supporto assistenziale. Per quanto sia ancora in fase di sviluppo sono state evidenziate tempistiche inerenti all'uso degli ultrasuoni come supporto per l'impianto di cateteri venosi periferici, per il monitoraggio dello stato di volume del paziente analizzando le cavità pleuriche e dimensioni della vena porta e alla valutazione delle dimensioni vescicali. In merito ai cateteri venosi gli sudi scientifici analizzati non quantificano la durata del percorso formativo, ma spesso viene indicato un numero minimo di impianti di cateteri venosi impiantati da parte del discente sotto supervisione dell'operatore esperto, al fine di ottenere l'abilità ad effettuare queste procedure in autonomia. Nello studio Weiner (165) viene considerato autosufficiente l'operatore che esegue 5 impianti di cateteri venosi ecoguidati. Per quanto riguarda la valutazione del livello di instabilità attraverso il monitoraggio delle cavità pleuriche e della vena porta sono meno definite le tempistiche d'apprendimento ma si deduce che sia più rapito acquisire l'autonomia nel monitoraggio delle cavità pleuriche rispetto all'ottenere immagini efficaci della vena cava. $(166,167)$ Le tempistiche indicate dallo studio analizzato equivalgono in un training trimestrale per gli infermieri non aventi precedente esperienza in merito all'uso dell'ecografo prima della formazione. E' stata prevista la supervisione da parte di cardiologi ed i discenti erano tenuti ad apprendere la tecnica fornendo scansioni efficaci del pericardio e della pleura in autonomia durante il percorso formativo.(160) In un altro studio gli infermieri partecipanti provenivano dalla terapia intensiva aventi già esperienza nei tre anni precedenti per quanto riguarda l'uso dell'ecografo avendo effettuato più di 200 scansioni con supervisore, ma non avevano esperienza di scansioni su pleura e vena cava. Hanno partecipato alla formazione della durata di un mese finalizzata allo studio in oggetto, con supervisione di cardiologi, dove gli è stato richiesto di effettuare circa 15-20 scansioni a testa prima di iniziare il progetto sperimentale ed iniziare a raccogliere dati.(167) Rispetto alle metodiche di apprendimento per la valutazione vescicale non sono indicati percorsi specifici, ma è considerato percorso d'apprendimento breve.(168) In questo caso la sperimentazione ha arruolato infermieri aventi già esperienza di cateterismi con tecnica tradizionale, al fine dello studio hanno dovuto seguire un breve corso di 30 minuti di didattica finalizzata al riconoscimento delle immagini ecografiche ed ognuno di loro era tenuto ad effettuare la procedura con successo su almeno 5 bambini registrandone altresì le dimensioni vescicali corrette.(168)

\section{Somministrazione di terapia e chemioterapia}

La somministrazione della terapia è un processo complesso che comprende più fasi: approvvigionamento, immagazzinamento, conservazione, prescrizione, preparazione, distribuzione, somministrazione e monitoraggio.(169-174)

Durante ciascuna fase si possono verificare errori che possono mettere in pericolo la sicurezza del paziente. Secondo un rapporto dell'Institute of Medicine (IOM) statunitense ogni anno, per errori commessi dai professionisti sanitari, si stima che ci siano 1,5 milioni di eventi avversi da farmaci prevenibili $(169,175)$ e che ogni anno muoiano tra 44.000 e 98.000 cittadini americani, un numero di decessi che rappresenta la settima causa di morte negli Stati Uniti.(169)

Gli errori di terapia farmacologia sono eventi prevenibili ed evitabili e vanno differenziati dalle reazioni avverse ai farmaci (adverse drug reaction - ADR) legate al farmaco stesso e che vengono rilevate e valutate mediante le attività di farmacovigilanza nazionali, regionali e aziendali. L'errore può verificarsi nelle fasi di prescrizione, trasmissione della prescrizione, allestimento, confezionamento, etichettatura, distribuzione, dispensazione, somministrazione, monitoraggio ed educazione al paziente.(169-178)

Fondamentalmente vengono riconosciute queste categorie di errore di terapia farmacologica:

- errore di prescrizione, riguarda sia la decisione di prescrivere un farmaco sia la scrittura della prescrizione;

- errore di trascrizione/interpretazione, della terapia medica, riguarda la errata comprensione della totalità o anche solo di parte della prescrizione;

- errore di allestimento, riguarda la fase di preparazione o di manipolazione del farmaco prima della somministrazione; 
- errore di distribuzione, riguarda la fase in cui il farmaco viene distribuito dalla farmacia alle unità operative o ai pazienti

- errore di somministrazione, include la preparazione, il controllo e la somministrazione, il monitoraggio dell'efficacia del trattamento, la registrazione degli eventi avversi e l'informazione del paziente riguardo al farmaco che sta assumendo. Una somministrazione sicura richiede una preparazione corretta.

L'errore può essere commesso durante l'iter diagnostico terapeutico dal medico, dal farmacista, dall'infermiere, dal paziente. La normativa vigente assegna la prescrizione terapeutica alla competenza del medico, mentre all'infermiere è assegnata la somministrazione dei farmaci prescritti: l'infermiere garantisce la corretta applicazione delle prescrizioni diagnostico-terapeutiche (D.M. Sanità 14.09.1994 n. 739 , art. 1-comma 3).

All'interno dei processi di terapia rientra appieno, con livelli di complessità sempre più profondi nei processi di somministrazione e monitoraggio, la somministrazione di chemioterapia all'interno delle malattie oncologiche.

Al giorno d'oggi è possibile osservare un discreto aumento dell'incidenza dei tumori già a partire dai 55 anni ma in Italia si stima che oltre il $50 \%$ del totale dei tumori sia diagnosticato in individui con più di 70 anni. Si stima inoltre che il 35\% (oltre 918.000 soggetti) di pazienti con un'età superiore a 75 anni convivano con una diagnosi di cancro.(179) I principali approcci nel trattamento delle neoplasie sono ad oggi rappresentati dalla chirurgia, dalla chemioterapia, dalla radioterapia, dalla terapia ormonale, dalle terapie biologiche e dall'immuno-oncologia. La decisione sulla terapia da utilizzare, compresa la possibilità di più trattamenti in associazione, deve tenere conto di una serie di fattori: tipo di tumore, stadio e sue caratteristiche molecolari, storia clinica della persona, età, sesso, stato psicologico e condizioni generali del malato, ecc.(180)

Punto cardine dell'approccio dell'oncologia medica è costituito dalla chemioterapia. Con questo termine si intende il trattamento che si basa sulla somministrazione di sostanze chimiche (specifici farmaci definiti antiblastici), che distruggono le cellule tumorali e contrastano la loro crescita prevenendone e/o rallentandone la riproduzione al fine di debellare il tumore, bloccarne la crescita, impedire le recidive e/o limitare i sintomi associati ad esso.(181) Tali proprietà citotossiche (realizzate con plurimi meccanismi di azione) sono alla base dell'utilizzo dei farmaci antiblastici anche nel trattamento di patologie non oncologiche (quali artrite reumatoide, trapianto d'organi, talassemia, psoriasi, ecc), concorrendo alla diffusione del ricorso alla chemioterapia anche in ambienti non strettamente oncologici.(182,183) Di recente l'uso di tale pratica è aumentato significativamente anche sotto lo stimolo delle nuove conoscenze riguardo alla bio- logia dei tumori, ai metodi innovativi di target therapy su specifiche caratteristiche delle cellule tumorali, all'aumento dell'uso di farmaci antiblastici come adiuvanti all'approccio chirurgico o radioterapico, allo sviluppo di numerose e differenti vie di somministrazione rispetto alla classica via endovenosa.(184) Quanto detto giustifica ciò che dichiara l'Annuario Statistico Italiano del 2013 che, nella tabella riportante i primi 50 DRG (diagnosis related groups) per numerosità delle dimissioni e degenza media per acuti in regime ordinario, annovera al decimo posto la voce: Chemioterapia non associata a diagnosi secondaria di leucemia acuta.(185)

In relazione alla patologia, alla prognosi e all'obiettivo del trattamento, è compito del team multidisciplinare definire il piano di cura chemioterapico adatto per ogni paziente, anche in termini di frequenza e lunghezza del trattamento stesso. La terapia antineoplastica è spesso strutturata in regimi complessi e per periodi prolungati. Può essere somministrata con cadenza giornaliera, settimanale o mensile, ed è generalmente prevista ad intervalli regolari chiamati cicli che prevedano periodi di riposo dal trattamento al fine di rendere l'organismo del paziente in grado di ristabilirsi per poter affrontare il ciclo successivo (186) (sia in termini di rigenerazione cellulare, sia in termini di performance status, ovvero la capacità del soggetto di sostenere l'impatto della patologia e del trattamento sulle attività della vita quotidiana).(187) La via di somministrazione scelta per il trattamento, insieme alle risorse territoriali, concorre a determinare il setting di cura più adeguato ad ogni singolo caso e dipende da: farmaco, obiettivo terapeutico, tipo e localizzazione del tumore, paziente e proprie esigenze, livello di aderenza alla terapia.

Nel Libro Bianco dell'Associazione Italiana di Oncologia Medica 2015 che riflette la situazione dell'oncologia in Italia, sono state censite 319 strutture oncologiche: per quanto riguarda la somministrazione di chemioterapia, l'attività di queste strutture è principalmente distribuita tra reparti di day hospital (presenti nel 89,66\% delle strutture censite), di degenza ordinaria oncologica (presenti nel 59,25\%) e ambulatori (presenti nel 57,68\% dei casi).(188) Il fatto che vi siano meno reparti di degenza ordinaria rispetto a reparti di day hospital è giustificabile pensando che ad oggi la maggior parte dei pazienti oncologici riceve trattamento chemioterapico senza la necessità di ricovero, che il ricovero sia riservato ai casi di prima somministrazione o somministrazione di farmaci antineoplastici in continuo (188) e che il ricorso alla chemioterapia per trattamenti non antitumorali e/o somministrata per via orale (tipicamente scelta per pazienti anziani non candidati ad approccio endovenoso) non è considerato nell'indagine dell'AIOM e si verifica frequentemente in reparti di degenza ospedaliera afferenti all'area medica non oncologica (ad esempio nelle Medicine Generali).

Data la complessità della procedura e di questa categoria di pazienti è ormai parere condiviso che la che- 
mioterapia debba essere somministrata in aree individuate per tale scopo, che abbiano ambienti idonei per la manipolazione e la conservazione degli agenti chemioterapici. Soprattutto a livello internazionale è inoltre ritenuto indispensabile che la somministrazione degli agenti antiblastici venga affidata ad infermieri e staff medico che abbiano effettuato un programma formativo, siano stati valutati e ritenuti competenti (secondo quanto stabilito dai protocolli locali) e che abbiano effettuato l'aggiornamento obbligatorio.(183,184,189191) Qualora si debba ricorrere a chemioterapia in unità operative con personale non specificamente qualificato, è indicato che la procedura avvenga sotto la diretta supervisione di infermieri competenti in materia, che provvedano successivamente al supporto formativo dei colleghi inesperti.(183)

Tra i trattamenti farmacologici, quello chemioterapico è uno dei pochi i cui dosaggi terapeutici sono personalizzati e non standardizzati, essendo generalmente definiti sulla base di peso corporeo, body mass index e superficie corporea, e di indagini di laboratorio (es. funzionalità epatica e renale), elementi che spesso costringono ad un aggiustamento dei dosaggi di riferimento. Ad accrescere la complessità di tali terapie concorre il frequente uso di polichemioterapie in sequenza, la grande variabilità degli schemi terapeutici e l'uso di svariate vie e modalità di somministrazione (bolo, infusione continua, ecc.), che aumentano esponenzialmente il rischio di errore (basti pensare che alcuni farmaci hanno dosaggi terapeutici per una specifica via di somministrazione che risultano però potenzialmente fatali se somministrati in una via differente). Quanto detto, in associazione alla continua evoluzione nel campo della ricerca farmacologica, costringe gli infermieri a mantenersi continuamente aggiornati al fine di garantire un'assistenza sicura e competente.(192)

La crescente specializzazione riguardo al trattamento e alla assistenza a pazienti con cancro, associate alla pressione economica legata alla presenza di sistemi complessi per l'erogazione delle cure, stanno promuovendo la crescita del ruolo dell'infermiere in questo ambito.(193) Secondo la Canadian Association of Nurses in Oncology/Association Canadienne des Infirmieres in Oncologie (CANO/ACIO), data la grande variabilità di ruoli richiesti per assicurare una assistenza sicura e competente in ogni setting, solo infermieri che abbiano ottenuto almeno la qualifica di Registered Nurse sono idonei all'assistenza a pazienti sottoposti a chemioterapia,(184) poiché tale procedura è ritenuta ad alto rischio per il grave danno potenziale a carico del paziente, in caso di errori. Ad aumentare la complessità e le fonti di errore concorrono la presenza di diversi e numerosi regimi terapeutici e l'ampiezza del processo di gestione degli antiblastici (che partendo dalla ordinazione e prescrizione, giunge alla preparazione e somministrazione).(194) A livello europeo ed extraeuropeo, tuttavia, non esiste un univoco percorso di specializzazione postbase in questa direzione, portando alla creazione di nu- merose figure specializzate in relazione al contesto geopolitico, dai contorni difficilmente definibili.(193) Tra i ruoli riconosciuti in modo uniforme all'interno dell'UE all'infermiere di oncologia troviamo: ruolo clinico, di innovazione e sviluppo, di consulenza, di educazione, di ricerca, di coordinamento, di case manager e di leadership.(193)

Nel 2009, con il volume Chemotherapy and Biotherapy Guidelines and Recommendations for Practices, la Oncology Nursing Society (ONS) ha stabilito l'importanza della competenza infermieristica sulle abilità di base in oncologia per garantire la qualità dell'assistenza a pazienti oncologici e tra queste troviamo: somministrazione delle terapie antiblastiche secondo conoscenze aggiornate e abilità pratiche basate sulle recenti ricerche scientifiche e prove di efficacia. Gli aspetti su cui è ritenuta fondamentale la competenza sono: controllo e verifica del regime di trattamento chemioterapico, trascrizione e verifica dell'ordine, gestione in sicurezza dei farmaci antiblastici, somministrazione dei farmaci e corretta gestione di spandimento del farmaco. Sempre l'ONS ha inoltre previsto percorsi formativi per tutti gli infermieri che somministrano farmaci antiblastici, evidenziando percorsi educativi differenti in caso di infermieri che operano in setting con somministrazioni frequenti e/o alti dosaggi o in setting con somministrazioni meno frequenti e/o bassi dosaggi.(194,195) Dalla revisione della letteratura è emerso come l'infermiere che somministra chemioterapia debba avere, rispetto ai singoli aspetti: conoscenze specifiche ed approfondite, abilità pratiche e gestionali, capacità educative.

Gli aspetti presi in considerazione sono:

- principi generali di chemioterapia (biologia delle cellule tumorali, cinetica delle cellule normali e maligne, classificazione e meccanismo di azione, selezione del farmaco) e conoscenze e competenze specifiche sulla gestione degli agenti antitumorali utilizzati abitualmente nello specifico setting di cura e loro manipolazione (obiettivi del trattamento, conoscenza dei trattamenti standard e dei protocolli di ricerca);(184)

- principi e abilità per una somministrazione sicura attraverso tutte le vie e modalità utilizzate nella specifica sede (184) con particolare attenzione a:

- chemioterapia orale (interventi educativi a paziente e caregiver riguardo a regola delle $7 \mathrm{G}$, modalità di corretta assunzione, interazione con cibi/farmaci/stile di vita, effetti collaterali comuni ed effetti collaterali che necessitano di comunicazione tempestiva a medico/infermiere, gestione di eventuali dosi saltate/errate); $(184,196,197)$

- valutazione del grado di aderenza terapeutica, rivalutazione periodica e monitoraggio continuo del paziente (comprensivo di raccolta dei feedback, educazione della persona e/o caregiver e interventi di counseling, sistemi di supporto e promemoria);(184,198,199) 
- chemioterapia endovenosa (specifiche tecniche di somministrazione e di dosaggio del farmaco e delle tossicità; piani terapeutici e principi alla base delle terapie combinate; educazione del paziente sulla gestione di eventuali device, ecc);(184,200)

- principi per il controllo della correttezza della prescrizione (paziente e diagnosi, ciclo di trattamento, regola delle G (Giusto farmaco, Giusta dose, Giusta via, Giusto orario, Giusto paziente, Giusta registrazione, Giusto controllo), allergie, necessità di terapie ancillari o idratazione) e del farmaco preparato dalla Unità di Manipolazione Farmaci Antiblastici (se presente), eseguendo il controllo a due operatori (nuovamente sull'identificazione del paziente - es. nome e data di nascita -, la regola delle $\mathrm{G}$ e il controllo dell'etichettatura); $(7,28)$

- principi e requisiti per una manipolazione sicura dei chemioterapici, dei relativi rifiuti e di eventuali liquidi biologici contaminati (dispositivi di protezione individuale appropriati rispetto alla via di somministrazione e in base agli standard nazionali, utilizzo di kit per lo spandimento e lo smaltimento dei rifiuti);(184)

- principi di misurazione, monitoraggio ed interpretazione di parametri e dati rilevanti per la somministrazione della terapia antiblastica (es. Performance Status, indagini di laboratorio quali funzionalità epatica o renale, ECG ecc.), spigando al paziente la ragione dei monitoraggi specifici;(184)

- principi di conoscenza, prevenzione, identificazione precoce, monitoraggio e gestione di tossicità ed eventi avversi associati a chemioterapia (e al singolo farmaco in particolare);(184)

- principi di conoscenza, prevenzione, identificazione precoce, monitoraggio e gestione di sintomi ed effetti collaterali specifici per la terapia posta in atto,(184) in particolare:

- nausea e vomito (fisiopatologia e fattori di rischio, linee guida aggiornate sulla loro gestione, ultimi sviluppi farmacologici e metodi di screening dei pazienti a rischio e di valutazione continua durante il trattamento, in modo da provvedere all'educazione di paziente e caregiver);(201)

- mucosite e diarrea (fisiopatologia e fattori di rischio, strumenti di monitoraggio e valutazione, complicanze, protocollo sull'igiene orale e modalità di trattamento, informazioni per paziente e caregiver riguardo a prevenzione, cibi sconsigliati, ecc.);(202)

- stravaso (modalità generali di gestione e quelle farmaco specifiche facendo riferimento a linee guida, procedure e protocolli ecc, basati sulle prove di efficacia; educare il paziente sui comportamenti da adottare per limitare il rischio di dislocazione del device e sull'importanza di una comunicazione tempestiva dell'eventuale comparsa di dolore, ecc.);(203)
- scelta, valutazione di appropriatezza e/o posizionamento e gestione dei device vascolari (periferici e centrali);(184)

- utilizzo e gestione del materiale necessario alla somministrazione (es. set di infusione, pompe infusionali) in modo appropriato rispetto al protocollo di trattamento, alle preferenze della persona e alle risorse disponibili;(184)

- principi di oncologia psicosociale e considerazioni culturali in relazione ai bisogni specifici della popolazione trattata (es. bambini) al fine di instaurare, mantenere una relazione terapeutica e di supporto con paziente e familiari/caregiver, che consideri contesto culturale, spirituale ed emotivo (paure e convinzioni sbagliate, eventuale barriera linguistica, capacità di reagire, altri aspetti specifici legati alla persona) e permetta di individuare l'eventuale necessità di supporto del team di cure palliative, o di sistemi sociali, ecc; $(184,204)$

- valutazione complessiva ed accurata delle persone sottoposte a chemioterapia, delle loro famiglie e dei loro bisogni di conoscenza, al fine di elaborare un percorso di cura personalizzato (per sviluppo di self-management e identificazione della necessità di interventi per l'eventuale sostegno);(184)

- valutazione della prontezza e della capacità di apprendere della persona (attraverso l'indagine su: età e livello di sviluppo, conoscenze di partenza, attese rispetto a malattia e trattamento, risposta alle nuove conoscenze, capacità e desiderio di autocura) e conseguente impostazione della velocità/passo di insegnamento riguardo al proprio schema di trattamento,(184) poiché quando il paziente riceve informazioni rilevanti, accurate ed attuali è facilitato a prendere autonomamente importanti decisioni in merito alla propria salute.(205) Perché il piano educativo sia realmente efficace è fondamentale prevedere metodi per il rinforzo degli interventi educativi (prevedendo anche materiale scritto cartaceo o elettronico per pazienti e caregivers - ed eventualmente per lo staff)(204) e continue valutazioni dei risultati da condividere con il gruppo interdisciplinare;(184)

- abilità nel riconoscere e analizzare con spirito critico le situazioni con problemi etici e legali potenziali, legati alla somministrazione di chemioterapia;(184)

- utilizzo idoneo della documentazione, (184) come parte integrante e specchio dell'assistenza infermieristica. Deve includere: raccolte dati, pianificazioni, interventi e valutazioni che riflettano outcomes (clinici, educativi, ecc.), conoscenze, giudizi significativi e il pensiero critico degli operatori sul singolo paziente, per garantire un' assistenza personalizzata durante tutto il processo terapeutico.(205)

- utilizzo, conoscenza e consultazione di linee guida, protocolli e procedure per l'uso di specifici farmaci;(184)

- principi e abilità per promuovere la continuità delle cure attraverso: il coordinamento del per- 
corso terapeutico e la collaborazione con altri professionisti, assicurandosi che le cure siano fornite nel setting più adeguato e sicuro in relazione ai bisogni della persona; l'agevolazione del processo di comunicazione tra la persona e il professionista sanitario di cui ha bisogno facendo da intermediario ove e se possibile (ad esempio spiegando le decisioni prese dall'equipe o dando indicazione di chi, come e quando chiamare in caso di necessità); l'organizzazione del rifornimento del materiale occorrente al paziente per il self-care (farmaci, aghi, pompe ecc) e aiutando la persona ad intraprendere eventuali percorsi di supporto (es. psicologico, sociale);(184,204)

- completamento di un programma formativo e utilizzo della ricerca e del sapere basato sulle prove di efficacia rispetto a persone sottoposte a chemioterapia, attraverso: ricorso alle evidenze nella pratica clinica, identificazione di problemi/quesiti di ricerca e di gap nell'assistenza, valutazione critica degli articoli, supporto ai trial clinici, lavoro in gruppi interprofessionali per cercare risposte evidence-based, partecipazione ad associazioni professionali di oncologia e registrando e pubblicando il proprio lavoro;(184,204)

- consapevolezza del limite delle proprie competenze evitando di gestire situazioni nella quali il professionista rileva le proprie carenze o incapacità ma, laddove competente, predisposizione ed insegnare in modo informale agli altri professionisti (nei reparti e negli ambienti di lavoro), ai nuovi membri dello staff, agli studenti e ai membri del team multidisciplinare.(204)

\section{Le lesioni da pressione}

Il rischio di sviluppare ulcere da pressione riguarda qualunque paziente allettato per brevi, medi o lunghi periodi. La letteratura mostra che i dati maggiori sono in riferimento a pazienti anziani fragili che sviluppano ulcere da pressioni a causa di immobilizzazione e allettamento, troviamo dati epidemiologici di lesioni che compaiono anche dopo interventi chirurgici in pazienti giovani $(<65$ aa) e bambini.(206,207) Digitando come parole chiave bedsores e guidelines su Medline i risultati sono molteplici e ogni azienda sviluppa protocolli interni e crea gruppi di ricerca per garantire un continuo aggiornamento sulle lesioni da pressione. La ricerca bibliografica svolta ha fatto emergere che le lesioni da pressione (LdP) sono un fenomeno presente, e sempre più in aumento per due fondamentali cause: l'aumento dell'età media dei pazienti e, soprattutto in Italia, la carenza, sempre più grave di personale all'interno dei reparti, che comporta un'impossibilità di mettere in atto tutte le manovre di prevenzione necessarie per diminuire il rischio di comparsa delle lesioni e non aggravare lesioni già presenti; in Italia i dati relativi ad uno studio nazionale di prevalenza che ha valutato 13.081 pazienti ricoverati in 24 strutture ospedaliere, ha documentato un tasso di prevalenza pari al 10,97\%, che non si associava, però, ad un'adeguata risposta in termini di applicazione di misure preventive, che infatti venivano adottate soltanto nel 9,4\% dei casi.(208)

Quando si parla di lesioni da pressione il primo passo è quello di saper riconoscere il tipo di lesione, classificarla e riconoscere precocemente l'eventuale presenza di infezione. Il passo successivo è conoscere le diverse cause e fattori che contribuiscono allo sviluppo di LdP: età (i più a rischio sono i bambini e gli anziani $>65 \mathrm{aa}$ ), l'immobilizzazione prolungata (immobilità $>3$ giorni), la pressione (il punto critico per lo sviluppo di un'ulcera si ha quando la pressione che comprime la superficie corporea sul piano di appoggio è maggiore della pressione del sangue nel distretto arteriolo-capillare $>32$ $\mathrm{mmHg}$,(209) le forze di stiramento, la frizione, l'aumento della temperatura cutanea, la malnutrizione, l'incontinenza urinaria e fecale, il fumo, la diminuzione della capacità cognitiva.(210) Tutti questi fattori devono essere individuati dall'infermiere per poter inquadrare una situazione clinica a rischio di sviluppare una lesione o di peggiorare una condizione già esistente. Fondamentale durante una valutazione iniziale è saper eseguire una diagnosi differenziale tra:

- eritema, che si presenta con un rossore che non scompare alla digito pressione;

- ferite croniche di altra natura (ulcera diabetica, ulcere vascolari);

- siti atipici per ulcere da decubito (superfici laterali degli arti, dorso del piede, punta delle dita).(211)

Dalla letteratura emerge che i tassi di prevalenza di sviluppare una LdP, variano dallo $0,38 \%$ al $53,2 \%$ e i tassi di incidenza variano dall' $1,9 \%$ al $71,6 \%$ in tutta Europa, Giappone, Cina, Medio Oriente, USA, Australia e Canada.(212)

Uno studio del 2013 ha rilevato una prevalenza delle LdP del 20,9\% durante i ricoveri ospedalieri (pazienti in acuto) e nelle lungodegenze del 11,7\%.(213)

Una revisione italiana del 2011 stima un'incidenza dallo $0,4 \%$ al $38 \%$ nei reparti ospedalieri, un'incidenza dal 2,2\% al 23,9\% nelle RSA, mentre nell'ambito domiciliare dallo $0 \%$ al $17 \%$.(214) La quinta survey del National Pressure Ulcer Advisory Panel (NPUAP),(215) ha documentato una prevalenza globale nei reparti per acuti del $14,8 \%$, identificando come fascia di età maggiormente colpita quella tra $\mathrm{i}$ 71 e gli 80 anni (tasso di prevalenza pari al 29\%).

È stato stimato che le ferite croniche colpiscano dai 3 ai 6 milioni di persone negli USA e di questi 1' $85 \%$ sono persone over 65 aa.(216) Il progetto S.I.U.C.(Studio Italiano Ulcere Cutanee) ha condotto uno studio sulle lesioni da pressione con l'obiettivo di raccogliere il maggior numero di dati sulle lesioni. Ne risulta che di 3975 casi esaminati, la maggior parte dei pazienti sia di sesso femminile, con un'età media di 77 anni, e con 1.66 lesione per paziente (più lesioni per paziente). Un dato significativo che emerge è la varietà eziologica delle lesioni: la sede maggiormente 
colpita risulta essere la gamba con il 53\%, a seguire il sacro con il $14,9 \%$, il piede $14,9 \%$, il trocantere $3 \%$, le dita $2,5 \%$, la zona ischiatica $1,4 \%$ e le altre sedi con il $10,4 \% .(217)$

La popolazione a rischio è rappresentata da pazienti anziani pluripatologici allettati totalmente o parzialmente, pazienti con patologie neurologiche e/o con lesioni al midollo spinale, pazienti ricoverati nelle unità intensive traumatizzati, ustionati o con gravi patologie, pazienti sottoposti a lunghi interventi chirurgici (superiori alle 4 ore), $(209,218)$ pazienti oncologici, cardiopatici, diabetici. Nella valutazione dei pazienti a rischio considerare: caratteristiche demografiche (età, sesso, razza), caratteristiche antropometriche (BMI), stato fisiologico (parametri vitali), stato nutrizionale, stato funzionale (continenza), ADL (activities of daily living), IADL (instrumental activities of daily living)), stato psicologico e cognitivo, comportamento sociale e aderenza terapeutica.(219)

All'ingresso in reparto del paziente eseguire una valutazione completa: raccolta anamnestica e stato di coscienza, ispezione della cute/esame obiettivo, compilazione di scale validate (BRADEN, NORTON), valutazione, dove possibile, del dolore con scale validate (VAS, VNS), valutazione nutrizionale.

La compilazione delle scale di valutazione non deve essere un'azione fine a se stessa, ma portare l'operatore ad ampliare il quadro di valutazione e mettere in atto una serie di azioni finalizzate alla prevenzione.(220)

La valutazione deve coinvolgere l'intera equipe multidisciplinare: infermiere, OSS, medico, dietologo, chirurgo vascolare, infettivologo ove le LdP sono state valutate come infette.(221)

Nella fase di prevenzione, prima di tutto eseguire l'esame obiettivo: 1'ispezione della cute è una fase fondamentale della presa in carico del paziente. Tra gli individui a rischio, le ulcere da pressione si sviluppano sulle aree corporee esposte a una pressione elevata senza interruzioni per periodi prolungati.(221) Se la pressione dell'interfaccia è superiore alla media pressione capillare, il flusso di sangue può essere compromesso, e le aree colpite possono diventare ischemiche e necrotiche. Un'area diventa ad alto rischio ischemico quando la pressione dell'interfaccia supera i 30-32 $\mathrm{mmHg}$, va sottolineato che la durata della pressione d'interfaccia è altrettanto importante quanto la grandezza della pressione d'interfaccia. Da questo nasce la necessità di utilizzare presidi antidecubito.(209)

La letteratura scientifica mostra che nei soggetti valutati a rischio di LdP, è ragionevole l'utilizzo di materassi antidecubito rispetto ai dispositivi standard di norma presenti nei comuni ambienti di cura. È fondamentale la decisione sul tipo di presidio antidecubito da utilizzare non basandosi solo sulle scale di valutazione che identificano il medio o alto rischio ma eseguendo una valutazione multidisciplinare. $\grave{\mathrm{E}}$ stato dimostrato che l'utilizzo dei presidi antidecubito riduce il rischio di sviluppare una $\mathrm{LdP}$ del $60 \% .(222)$
Successivamente alla scelta del presidio più idoneo per il soggetto, l'infermiere deve pianificare un piano di riposizionamento e mobilizzazione del paziente. Dopo aver considerato le condizioni del paziente e il livello di mobilità/attività vanno stabiliti la frequenza del riposizionamento (alcuni studi dimostrano che il riposizionamento deve essere effettuato ogni 2-3 ore) e gli orari di scarico della pressione. Limitare la posizione della testiera del letto a $30^{\circ}$ dove possibile, quando si adotta la posizione seduta assicurarsi che ci sia un piano di appoggio per i piedi e limitare il tempo in questa posizione. Per i soggetti con ulcera da decubito sacrale presente evitare la posizione seduta e dove necessaria limitare la posizione a 60 minuti al giorno massimo 3 volte. La posizione laterale è consigliabile durante le ore notturne.(223)

E' inoltre necessario valutare la presenza di dispositivi medicali che possono provocare un'ulcera. Eseguire un'ispezione della cute intorno al presidio medicale almeno due volte al giorno e rimuovere presidi non necessari che possono essere fonte di pressione, per il corretto sistema di fissaggio dei presidi seguire le indicazioni della casa produttrice.(223)

Infine la letteratura dimostra la correlazione tra malnutrizione, sviluppo di LdP e ritardata guarigione della ferita.(224) Per questo durante la presa in carico si deve eseguire uno screening nutrizionale (gli studi dimostrano che nel 39,5\% dei pazienti con LdP emerge una malnutrizione). La valutazione prevede: calcolo del BMI e successivo controllo periodico del peso (dove possibile), anamnesi remota e prossima, l'utilizzo di scale validate (MNA, mini nutritional assessment, test di MUST) e la valutazione della capacità del soggetto di alimentarsi autonomamente. Per i soggetti considerati a rischio di malnutrizione va programmata una visita con il dietista e un team interprofessionale di nutrizionisti per una valutazione completa e l'eventuale introduzione di nutrizione parenterale/enterale.

È possibile riassumere il trattamento in queste fasi: $(208,211,221,225)$ :

- Stadiazione della LdP e valutazione della delle zone perilesionali;

- Detersione della lesione con applicazione di soluzione fisiologica a pressione (utilizzare siringa con ago 19 Gauge);

- Toilettatura chirurgica, sbrigliamento meccanico, enzimatico, autolitico;

- Applicazione della medicazione secondo la stadiazione, mantenere sempre un fondo umido;

- Gestione del dolore;

- Decompressione della zona lesionata;

- Eventuale intervento chirurgico plastico ricostruttivo.

\section{Il dolore}

Il dolore è definito come un'esperienza sensoriale ed emotiva spiacevole associata a danno tissutale reale o potenziale. Questa definizione riconosce sia 
la natura fisiologica sia quella affettiva dell'esperienza del dolore.(226)

In tutto il mondo, il dolore scarsamente gestito o non rilevato rappresenta un peso per la persona, il sistema sanitario e la società. Il $18,9 \%$ della popolazione dei paesi industrializzati vive con il dolore, senza contare che in tutto il mondo vengono diagnosticati ogni anno 10 milioni di nuovi casi di cancro.(227) La prevalenza di un dolore persistente è stata dimostrata crescere con l'aumentare dell'età, raggiungendo il $65 \%$ nella popolazione adulta anziana ( $>65 \mathrm{anni}$ ), ma una gestione inadeguata è presente ed evidente anche fra neonati, bambini e adolescenti.(226)

Un'efficace gestione del dolore è un diritto della persona e la valutazione, gli interventi per alleviarlo, il monitoraggio, la prevenzione e riduzione, dovrebbero essere le priorità della cura del malato, indipendentemente dalla loro diagnosi o tipologia di dolore.(226) La sua gestione è considerata come un'importante parte dell'assistenza, così l'American Pain Society definisce il dolore come quinto parametro vitale per enfatizzare il suo significato e suggerire che l'accertamento del dolore dovrebbe essere automatico come misurare pressione arteriosa $\mathrm{o}$ polso.(228)

Il dolore è qualsiasi cosa la persona dica esso sia; esiste quando la persona che lo sperimenta dice che esiste. Questa definizione enfatizza la natura altamente soggettiva del dolore.(228) Esso è multidimensionale caratterizzato da componenti sensoriali, cognitive e affettive. La sua gestione deve essere incentrata sulla persona, tenendo in considerazione i fattori bio-psicosociali, spirituali, culturali, attraverso l'intervento di un team multiprofessionale. L'infermiere interviene ed agisce con il malato verso una gestione appropriata, in quanto deve possedere le competenze necessarie per valutare e gestire il dolore, incluse conoscenze, tecniche, abilità nella gestione in pazienti che non hanno possibilità di esprimersi.(226) L'infermiere collabora con gli altri professionisti sanitari mentre somministra trattamenti per il sollievo dal dolore, valuta la loro efficacia, educa l'assistito e famiglia insegnando loro come gestire i regimi per il controllo del dolore.(228)

Come enuncia l'articolo 34 del Codice Deontologico, l'infermiere si attiva per prevenire e contrastare il dolore e alleviare la sofferenza. Si adopera affinché l'assistito riceva tutti i trattamenti necessari.(229)

La letteratura (226-230) fornisce indicazioni relative al processo di educazione e formazione continua del personale di cura operante nelle strutture sanitarie ed alla informazione e sensibilizzazione della popolazione, consentono la realizzazione a livello regionale di progetti indirizzati al miglioramento del processo assistenziale specificatamente rivolto al controllo del dolore e prevedono la formazione di un Comitato Ospedale senza Dolore all'interno delle Aziende Ospedaliere, di cui il personale infermieristico deve costituire almeno un terzo dei membri.(230)

Nel verificare la presenza o il rischio di manifesta- zione, di ogni tipologia di dolore, è importante interrogare direttamente la persona, la famiglia o i care givers, avvalersi di strumenti per la rilevazione, osservare il paziente.(226)

Monitorare, risposte comportamentali:

- cambiamenti nel comportamento;

- cambiamenti nell'andatura;

- diminuzione dei livelli di attività;

- diminuzione del sonno (e della qualità del sonno) e dell'appetito;

- aumentata o ricorrente irrequietezza e agitazione;

- alterate espressioni facciali;

- perdita di funzionalità ed energie;

- movimenti di resistenza durante l'assistenza, posizioni di difesa;

- pianto, vocalizzazioni.(228)

Monitorare, risposte fisiologiche alla presenza di dolore:

- Aumento della pressione del sangue;

- Aumento della frequenza cardiaca;

- Aumento della frequenza respiratoria;

- Alterazioni metaboliche e neuroendocrine come aumento del consumo di ossigeno, aumento della glicemia, degli acidi grassi liberi, del lattato nel sangue.(228)

Se non vi è un'alterazione dei parametri vitali, tuttavia, non significa che il dolore sia assente.(231)

La valutazione del dolore deve essere regolare, sistematica e documentata. Condurre un'intervista iniziale per determinare le caratteristiche e cause del dolore del paziente, raccogliere dati utili alla pianificazione, dunque, è il primo passo da effettuare:

- Anamnesi del paziente, diagnosi e comorbidità (diabete, insufficienza renale, insufficienza epatica, ansia, depressione, ecc.) che potrebbero contribuire al dolore, pregressi interventi chirurgici; $(226,231,232)$

- Intensità del dolore, utilizzando una scala numerica, verbale o visiva, adeguata alle condizioni del paziente;

- Caratteristiche del dolore (durata, frequenza, localizzazione, irradiazione, quando è iniziato, in che modalità, come varia nel tempo) e qualità del dolore (pungente, pulsante, ecc.);

- Utilizzare scale o strumenti adeguati per i pazienti non in grado di rispondere alle domande;

- Fattori che aumentano o diminuiscono il dolore;

- Valutare come il dolore interferisce nelle attività quotidiane del paziente, sulla sua qualità di vita, sul sonno, sull'attività sessuale;(232)

- Valutare la terapia antidolorifica (dosaggio, frequenza delle somministrazioni, tipologia di trattamento);

- Valutare l'aderenza alla terapia, quanti farmaci sono assunti e come (alcuni pazienti preferiscono tollerare un certo livello di dolore per evitare gli effetti collaterali di alcuni antidolorifici);

- Registrare tutti i trattamenti eseguiti per il controllo del dolore, compresi trattamenti non farmacologici, 
complementari e di medicina alternativa;(233)

- Registrare l'efficacia di specifici farmaci o altre terapie utilizzate in passato;(233)

- Registrare l'interruzione di determinate terapie o trattamenti per inefficacia;(234)

- Registrare l'eventuale presenza di allergie a farmaci;(226)

- Valutare ed esplorare credenze e cultura della persona, età, livello d'istruzione, etnia, spiritualità; (226)

- Valutare la presenza o meno di care givers, il ruolo sociale, impiego;

- Valutare la presenza o storia di abuso di alcol o sostanze stupefacenti. $(231,232)$

Una gestione efficace del dolore richiede un approccio altamente individualizzato: (231)

- Collaborare con il paziente per identificare gli obiettivi per gestire al meglio il dolore, con strategie adatte per assicurare un approccio comprensivo al piano di cura;

- Stabilire un piano di cura che incorpori gli obiettivi della persona e del team multiprofessionale.(226)

La gestione del dolore dovrebbe basarsi sul principio del massimizzare l'efficacia e minimizzare gli effetti avversi rispetto la terapia farmacologica, includendo:

- Conoscere i principali analgesici utilizzati e i loro effetti (oppioidi, non oppioidi, adiuvanti);

- Valutare con il medico una variazione degli oppioidi (rotazione e/o dosaggio) quando necessario;

- Prevenire, valutare e gestire gli effetti avversi della terapia analgesica e i rischi dell'assunzione di farmaci oppioidi (depressione respiratoria, nausea, vomito stipsi, tolleranza, dipendenza);

- Valutare ogni intervento non farmacologico (fisico o psicologico) riguardo la sua efficacia e le potenziali interazioni con la terapia farmacologica in atto;

- Anticipare e trattare il dolore prima, durante e dopo trattamenti diagnostico-terapeutici invasivi e/o dolorosi; $(231,235)$

- Coinvolgere la persona, la sua famiglia, i care givers rispetto il piano di cura e le strategie da attuare.(226)

- Quando il dolore non è controllato con la terapia farmacologica tradizionale, valutare con il medico e specialisti metodologie avanzate come la PCA (patient controlled analgesia), iniezioni epidurali, intratecali, interruzione chirurgica dei percorsi del dolore (cordotomia, rizotomia).(226)

I farmaci analgesici sono il più comune approccio per la gestione del dolore. Sebbene gli analgesici siano prescritti dal medico, l'infermiere è responsabile della loro somministrazione, della valutazione della loro efficacia, dei loro effetti avversi e di segnalare al medico se è stato o meno ottenuto un adeguato sollievo. $(226,229,236)$

Gli interventi non farmacologici, di solito, possono aiutare ad alleviare il dolore con bassi rischi per l'assistito. Sebbene queste misure non sostituiscano i far- maci, possono essere tutte appropriate per gestire episodi di dolore, anche in combinazione con i farmaci. L'efficacia varia da soggetto a soggetto, in base anche a suoi vissuti ed esperienze. Poiché molti degli interventi non farmacologici non richiedono una prescrizione medica, l'infermiere può indipendentemente attuarli in collaborazione con il paziente e care giver.(236) Comprendono:

- Stimolazione cutanea e massaggio: promuove benessere e rilassamento muscolare; $(237,238)$

- Terapie con caldo e con il freddo: da applicare con attenzione, con un monitoraggio continuo, per evitare lesioni cutanee;

- Stimolazione nervosa transcutanea (TENS): degli elettrodi vengono applicati alla cute per produrre sulla parte dolorante una sensazione di pizzicore, vibrazione o ronzio; $(237,238)$

- Distrazione: deviare l'attenzione dell'assistito su qualcosa che non è il dolore (comprende attività semplici come ascoltare musica fino a attività fisica (quando possibile) e esercizi mentali;(237-239)

- Tecniche di rilassamento: un esempio è la respirazione lenta e guidata;

- Altre tecniche cognitive come immaginazione guidata, ipnosi, meditazione. $(237,238)$

- Agopuntura.(237)

E'essenziale sviluppare dei criteri di risultato personalizzati per ciascun assistito e rivalutare la risposta del paziente agli interventi attuati utilizzando gli stessi strumenti di valutazione, o comunque lo strumento più adatto alla condizione del malato (es. scale).(226) La rivalutazione deve determinare:

- La presenza di dolore;

- L'intensità;

- Stabilità delle condizioni mediche della persona;

- Tipologia di dolore (es. acuto o persistente)

- L'eventuale presenza di effetti collaterali.(231)

La valutazione della presenza di dolore deve avvenire regolarmente e frequentemente, entro un'ora dalla somministrazione, in seguito almeno ogni 4 ore.(231)

Comunicare e documentare le risposte della persona al piano di gestione del dolore. $(226,233)$

Una buona valutazione è il presupposto per un buon trattamento.

L'utilizzo delle scale trova spazio nella valutazione del dolore non solo in pazienti anziani con alterazioni cognitive (es. demenza avanzata) ma più ampiamente in persone che hanno difficoltà nell'esprimersi o non possono farlo, come neonati, bambini, persone con disabilità intellettive, pazienti critici o non coscienti, pazienti terminali.(226)

Una positiva relazione infermiere-assistito e l'educazione sono la chiave per gestire l'analgesia nelle persone con dolore. Nella preparazione per la gestione del dolore a domicilio l'assistito e la famiglia devono essere educati e guidati su quale tipo di dolore e malessere attendersi, quanto durerà il dolore e quando il dolore indica un problema che deve essere riferito. L'infermiere offre informazioni spiegando 
quali sono i comportamenti più adatti da praticare:(226,231,234)

- La persona e la famiglia devono comprendere lo scopo di ciascun farmaco, il momento appropriato in cui usarlo, gli effetti collaterali, le strategie che possono essere adottate per prevenirli; $(231,234)$

- Educare paziente, famiglia ad utilizzare la terapia analgesica come profilassi prima e dopo procedure dolorose e invasive;(231)

- Educare paziente e famiglia su problematiche come la dipendenza;(240)

- Educare il paziente ad assumere la terapia secondo uno schema regolare per evitare che il dolore aumenti con conseguente difficoltà nell'alleviarlo; $(231,234)$

- Educare il paziente e care givers ad utilizzare anche strategie non farmacologiche per il controllo del dolore, come tecniche di rilassamento, massaggi, terapie complementari;(231)

- Offrire alla persona e alla famiglia l'opportunità di esercitarsi nell'assunzione e somministrazione dei farmaci;(231)

- Sottolineare la necessità di tenere gli analgesici fuori dalla portata di bambini.(240)

Un'adeguata educazione all'autocura previene il rischio di un nuovo ricovero ospedaliero.(226)

\section{Gli accessi vascolari}

L'approccio tradizionale al posizionamento di un accesso vascolare, spesso, erroneamente, prevede la venipuntura del paziente fino all'esaurimento del suo patrimonio venoso. Quest'ultimo andrebbe valutato, preferibilmente, prima che si inizi la somministrazione di terapie parenterali.

La scelta del giusto device minimizza il numero di dispositivi utilizzati, i tentativi di inserimento, i rischi e il disagio del paziente; $(241,242)$ inoltre, un corretto inserimento e un'adeguata gestione del catetere, secondo le più recenti raccomandazioni bibliografiche, consentono la miglior riuscita della procedura.

Occorre premettere che, nel paziente con necessità di un accesso venoso, la scelta del presidio appropriato si basa essenzialmente sull'uso cui l'accesso è destinato, sulla tipologia di farmaci o soluzioni che verranno infuse per via endovenosa, sull'ambito nel quale l'accesso verrà utilizzato (intra o extra ospedaliero) e sulla durata per la quale è previsto. $(241,243,244)$

Si definiscono propriamente accessi venosi centrali tutti quei devices intravascolari la cui punta arriva alla giunzione tra la vena cava superiore (VCS) e l'atrio destro. Posizione considerata ideale per consentire un'infusione sicura di soluzioni di qualunque pH e osmolarità e che permette il monitoraggio emodinamico (misurazione della pressione venosa centrale e della saturazione di ossigeno nel sangue venoso misto).

Sono considerati centrali anche quegli accessi la cui punta è posizionata in vena cava inferiore, consen- tendo l'infusione di qualsiasi farmaco, ma non il monitoraggio emodinamico.

Tutti gli accessi venosi la cui punta non è allocata nelle due posizioni descritte devono essere considerati accessi periferici. $(241,244)$

Nell'ospedalizzazione vengono riconosciuti appropriati soltanto gli accessi venosi a breve termine (agocannule, cannule periferiche lunghe, CICC - centrally inserted central catheter - non tunnellizzati) o a medio termine (Midline, PICC - peripherally inserted central catheter -, CICC tunnellizzati non cuffiati), laddove è prevista una terapia infusiva per più di 6 giorni e/o in caso di scarso patrimonio venoso.(241, 245,246)

L'accesso venoso centrale di prima scelta dovrebbe essere il PICC, che ha caratteristiche ottimali di stabilità e consente l'infusione di alti flussi (fino a $5 \mathrm{~mL} / \mathrm{sec}$ ), di mezzo di contrasto e di soluzioni con qualsiasi pH ed osmolarità.(245,246)

Il suo posizionamento risulta vantaggioso in quanto garantisce un minor traumatismo rispetto ai CICC, e l'inserzione è facile e sicura. Il materiale in poliuretano è qualitativamente superiore, in termini di biocompatibilità. $(241,245)$

Se è vero che il PICC dovrebbe essere considerato l'accesso venoso centrale di prima scelta nel paziente ospedalizzato, è anche vero che vi sono determinate situazioni cliniche che richiedono obbligatoriamente il posizionamento di un CICC.

Le più frequenti condizioni sono:(241)

- Presenza di controindicazioni al posizionamento in sede brachiale (pregresso svuotamento linfonodale, alterazioni articolari o cutanee, paresi, indisponibilità di vene di calibro sufficiente, trombosi venosa, ecc.);

- Necessità di un accesso a quattro o cinque lumi;

- Necessità di un accesso venoso centrale in emergenza.

I dispositivi per accesso venoso possono essere inseriti mediante:

- Venipuntura diretta sotto controllo visivo e/o palpatorio;

- Venipuntura cieca (blind), utilizzando come guida reperi anatomici (determinate vene profonde del collo o della regione inguinale);

- Venipuntura ecoguidata;

- Venolisi chirurgica;

- Con visualizzazione/evidenziazione con tecnologia a raggi dello spettro vicino all'infrarosso.

La tecnica può essere, dunque, più o meno traumatica, e più o meno accurata, influenzando in modo decisivo la possibilità di successo dell'incannulamento, l'eventuale incidenza di complicanze all'inserzione e il rischio di trombosi venosa catetere-correlata.(241)

L'approccio blind è all'origine di malposizionamenti, tentativi ripetuti, sofferenze per il paziente, frustrazione per l'operatore, complicanze immediate e morbilità con ulteriore riduzione del patrimonio venoso utilizzabile.(247) 
L'utilizzo dell'ecografo come supporto alle procedure vascolari è uno dei temi preminenti e maggiormente fertili dell'ecografia infermieristica. Un approccio tecnologico (ecoassistito o ecoguidato), gestito dell'infermiere, che rappresenta il superamento di un gap operativo atto a migliorare l'assistenza ai nostri pazienti. $(247,248)$

La puntura ecoguidata permette inoltre di aumentare a quasi il $100 \%$ le possibilità di successo della manovra, minimizzando o azzerando i fallimenti e il danno accidentale a strutture arteriose o nervose vicine, che grazie all'ecografo possono essere identificate ed evitate. $(241,249,250)$

Inoltre, la visualizzazione delle vene con l'ecografo permette di misurarne il diametro interno, consentendo quindi di scegliere in modo appropriato il calibro del catetere, strategia che è alla base della riduzione del rischio trombotico. In tal modo, è possibile seguire la regola, ormai accettata a livello internazionale, che consiglia il posizionamento di un catetere in una vena il cui diametro interno sia almeno il triplo del diametro esterno del catetere. $(241,248,251)$

Questo approccio può essere applicato a qualsiasi vena che sia identificabile ecograficamente.(241)

\section{Il paziente fragile}

La fragilità è una sindrome fisiopatologica specifica, caratterizzata dalla presenza di almeno tre di cinque caratteristiche (perdita di peso non intenzionale, astenia, ridotta attività fisica, alterazione della velocità del cammino e debolezza muscolare) la cui presenza può associarsi ad alterazioni specifiche di molte variabili fisiologiche e biologiche, come i marker dell'infiammazione, della coagulazione, del metabolismo e anche endocrinologici.(252)

Riconoscere le caratteristiche della fragilità e comprendere le sue determinanti fisiopatologiche è stato un punto centrale della ricerca gerontologica degli ultimi anni. In particolare, la ricerca si è concentrata sull'identificazione di marcatori biologici che permettano uno screening della fragilità in fase precoce, quando vi sono maggiori possibilità di prevenzione e intervento.(253) La fragilità, essendo quindi un accumulo di deficit età associati di natura biologica, funzionale e sociale, può essere misurata da un indice, frailty index, che numericamente esprime quantitativamente il numero di tali deficit nel singolo individuo.(252)

La fragilità può essere scatenata e incrementata dallo stress, dalla depressione, dalla diminuita attività fisica e dalla ridotta assunzione di apporti proteici e di micronutrienti, allo stesso tempo però vengono associati fattori come l'isolamento sociale, l'abuso di alcolici, il fumo di tabacco, le malattie croniche e la polifarmacoterapia. La fragilità viene però riconosciuta attraverso cinque fattori clinici, dei quali per essere considerata tale devono esserne presenti almeno tre di questi:
- la perdita di peso (maggiore di 4,5 kg nell'ultimo anno);

- l'affaticamento (presente da almeno 3 giorni o 1 settimana);

- la riduzione della forza muscolare ( $<$ di $5,85 \mathrm{~kg}$ per l'uomo e $<$ di 3,37 kg per la donna);

- la ridotta attività fisica, valutabile attraverso la scala PASE (physical activity scale for the elderly)

- la riduzione della velocità del cammino ( $>7$ secondi per percorrere 5 metri su percorso noto).(253)

La debolezza fisica, associata al concetto di fragilità, è causata dalla perdita di massa muscolare, più comunemente conosciuta come sarcopenia, risultato del cambiamento anatomico e biochimico del muscolo e conseguenza dell'invecchiamento. I meccanismi alla base dello sviluppo della sarcopenia sono molti e includono lo stress ossidativo, la disfunzione delle citochine infiammatorie e degli ormoni, la malnutrizione, l'immobilizzazione e l'apoptosi muscolare. Tutti questi fattori possono contribuire allo sviluppo di fragilità.(254) Sono stati inoltre riscontrati in anziani fragili, confrontati con anziani non compromessi, livelli significativamente alti di marcatori infiammatori, come la proteina $\mathrm{C}$ reattiva (PCR) e l'interleuchina 6 (IL-6), ma anche markers endocrini e infiammatori, come il fattore VIII, l'emoglobina e l'ipertonicità plasmatica.(255) Il fatto che la lista di tali molecole sia piuttosto lunga e che l'associazione con l'outcome clinico non sia molto forte, rinforza l'ipotesi che non un solo marcatore biologico sia sufficiente a determinare i molteplici aspetti della fragilità.(253)

Le complicanze più frequentemente insorte nel paziente fragile ospedalizzato sono:

- Il delirium;

- L'allettamento;

- La malnutrizione e la disidratazione;

- Le infezioni.

Il delirium è la complicanza principale dell'ospedalizzazione negli anziani, interessa il 25$60 \%$ degli anziani ospedalizzati negli Stati Uniti e ha un costo di circa 8 miliardi di dollari all'anno. Il delirium consta in alterazioni del neurotrasmettitore $\mathrm{e}$ può essere associato a ipossia, infezioni, squilibrio elettrolitico, costipazione e dolore. Fondamentale nell'anziano ospedalizzato bisogna prevenire la confusione mentale, chiedendo i motivi per cui si è stati ricoverati, eseguire esercizi mentali per mantenere la memoria temporale e se notati cambiamenti umorali, documentarli e discuterne in equipe multidisciplinare. Importante è definire le cause del delirio e l'utilizzo di alcuni farmaci può sia prolungare che peggiorare tale condizione. Il delirium è prevenibile utilizzando adeguati farmaci antidolorifici a giusti dosaggi, evitando le ore di sonno nelle ore diurne, incoraggiando la famiglia a visite più frequenti, svolgendo attività di musicoterapia ed arteterapia e creando un ambiente tranquillo per il sonno durante la notte.(256) 
L'allettamento è risaputo essere fattore negativo per la ripresa dell'anziano fragile, poiché può causare ipotensione ortostatica, atelectasia, riduzione della forza muscolare, incontinenza, ulcere da pressione e confusione mentale. Il mantenimento dell'attività fisica previene l'insorgenza di cadute. Fondamentale è la valutazione dell'equilibrio e dell'andatura durante il cammino, la valutazione attenta degli spostamenti dal letto ai servizi igienici, accompagnando il paziente, garantendo inoltre un ambiente sicuro. Altri accorgimenti possono essere: il mantenimento del campanello a portata del paziente e posizionare il letto ad altezza minima, l'utilizzo di luci soffuse durante la notte e distanziare il più possibile attrezzature non sicure.

Almeno il $50 \%$ degli anziani ospedalizzati ricoverati soffrono di malnutrizione e disidratazione. Entrambe le condizioni sono correlate a cadute, delirium, lesioni da pressione, infezioni, aumento della degenza e decesso. Valutare lo stato nutrizionale nell'anziano è fondamentale e ancor di più lo è correggere la malnutrizione, per cui bisogna valutare la deglutizione, evitare lunghi digiuni e stimolare una corretta alimentazione. Ancor più importante è la prevenzione della stitichezza, per cui bisogna stimolare a bere e mangiare frutta e verdura. L'utilizzo del SNG deve essere valutato attentamente e solo in ultima analisi. Accorgimenti per prevenire la malnutrizione e la disidratazione sono: prediligere cibi richiesti dal paziente, fare spuntini gustosi ma ridotti, eseguire accurate igieni del cavo orale per prevenire infezioni e far assumere i pasti al tavolo e non a letto, così da evitare indigestione.

Gli anziani con ridotta funzionalità immunitaria sono a maggior rischio di sviluppo di infezioni. Le polmoniti e le infezioni delle vie urinarie comportano il ricovero ospedaliero con conseguente terapia antibiotica. Il trattamento delle infezioni può risultare complesso nell'anziano che ha già polipatologie, comportando una maggiore resistenza e ridotta efficacia delle terapie assunte. Alcune abitudini e manovre in corsia possono contribuire ad aumentare l'insorgenza di infezioni, infatti bisogna evitare il riposo a letto per lunghi periodi e il posizionamento del catetere vescicale deve essere attentamente valutato, e se necessario, mantenuto per periodi brevi. Importante è l'igiene personale totale ed eseguire un accurata igiene delle mani, anche da parte dei visitatori sia prima che dopo il contatto con il paziente.(256)

La riduzione della fragilità dovrebbe fornire grandi benefici per il paziente, per la famiglia e anche per la società. Clinicamente la fragilità deve essere identificata tramite strumenti di valutazione. Dalla letteratura si denota che quattro sono i trattamenti potenzialmente efficaci per il trattamento della fragilità: l'esercizio fisico, il supporto calorico e proteico, la vitamina $\mathrm{D}$ e la riduzione della polifarmacoterapia. È scientificamente provato che l'esercizio fisico migliora i risultati relativamente alla mobilità e alla capacità funzionale, infatti sarebbe utile proseguire dopo la dimissione con esercizi fisici semplicemente svolgibili al domicilio. Per quanto riguarda la perdita di peso è risaputo che aumenta la componente fragile, infatti incrementare il supporto calorico e proteico favorisce un aumento di peso e riduce notevolmente la mortalità. Inoltre l'integrazione proteica aumenta la massa, la forza ed il tono muscolare. La vitamina $\mathrm{D}$, deve essere di supplemento per il paziente fragile carente, poiché riduce il numero di cadute e la mortalità, anche se non ci sono fondamenti ben definiti. Infine, per quanto riguarda la polifarmacoterapia, è risaputo essere contributo importante della fragilità negli anziani, ridurre le terapie potrebbe diminuire sia la patogenicità della problematica e allo stesso tempo ridurre $\mathrm{i}$ costi.(257)

L'ospedalizzazione è risaputo essere la causa più importante del declino del paziente fragile, comportando un aumento di disabilità, riducendo la prognosi e incrementando la mortalità. Le cure ospedaliere sono fondamentali per la ripresa funzionale causa del ricovero ospedaliero, ma proprio quest'ultimo potrebbe incrementare il declino funzionale, comportando nuove disabilità nelle attività di vita quotidiana (ADL). Per ADL sono incluse le attività di lavarsi, vestirsi, spostarsi dal letto alla sedia, andare ai servizi, mangiare e camminare. Il fattore principale che predispone l'anziano al declino funzionale, indipendentemente dal motivo di ricovero, è l'immobilizzazione.(258)

Durante il ricovero si dovrà quindi correggere lo stile di vita del paziente, essendo questa condizione che comporta la prevenzione della fragilità. Le condizioni sfavorevoli sono: l'obesità, il fumo, le carenze dietetiche, la politerapia farmacologica, la carenza di vitamina $\mathrm{D}$ e l'inattività. Poiché la fragilità si manifesta con la progressiva riduzione della forza muscolare e del peso corporeo, le principali strategie utili a prevenirne l'incremento possono essere l'individuazione di attività fisiche-motorie e il controllo dell'alimentazione. Una revisione Cochrane del 2009 (259) incoraggia gli anziani a svolgere attività fisica aerobica semplice come camminare $\mathrm{e}$ in seguito aggiungere allenamenti con esercizi di resistenza. Numerosi dati in letteratura sottolineano che le persone che effettuano esercizi aerobici (camminare, nuotare, correre, ecc.) hanno una speranza di vita maggiore e un minore declino funzionale rispetto a chi ha una vita sedentaria, e allo stesso tempo si possono trarre benefici anche nel proprio stato d'umore e nelle proprie funzioni cognitive. Un altro parametro indicante fragilità è la perdita involontaria di peso superiore al 5\% nell'arco di un anno. Le linee guida dell'American Dietetic Association (2009) forniscono raccomandazioni utili per un efficace terapia medica nutrizionale finalizzata all'aumento dell'energia attraverso l'assunzione di nutrienti e proteine, al miglioramento dello stato nutrizionale complessivo e della qualità di vita. 
Individuare quindi i rischi correlati alla fragilità è fondamentale per prevenirne la problematica. Molte sono le scale validate che possono aiutare gli infermieri a determinare i rischi:

- Scala della depressione in geriatria;

- Scala per la valutazione delle attività di vita quotidiana (IADL);

- Mini Mental State Evaluation (MMSE);

- Mini-Cog Test;

- Mini Nutritional Assessment (MNA).(253)

I bisogni delle persone affette da malattie croniche sono complessi e la risposta deve essere mirata, focalizzata anche sull'anticipazione e la prevenzione delle complicanze, abbandonando il modello assistenziale $a$ domanda, tipico della prima metà del '900 e delle patologie acute.(260) Tutti i portatori di bisogni assistenziali complessi sociosanitari necessitano di un modello assistenziale il cui obiettivo sia quello della presa in carico e della continuità delle cure. Dalla presa in carico derivano percorsi sanitari di cura per setting assistenziali (domiciliari, semiresidenziali e residenziali), che hanno lo scopo di portare alla guarigione o stabilizzazione dei problemi di salute. Fondamentale per la continuità assistenziale è una dimissione protetta dall'ospedale al domicilio o in una struttura residenziale in modo tale da assicurare la prosecuzione dei trattamenti terapeutici per non interrompere i benefici acquisiti.(261)

L'accesso ai servizi per anziani è fortemente condizionato dai Sistemi Sanitari di riferimento. In Italia si elabora una strategia di valutazione globale delle problematiche espresse dall'anziano, la Valutazione Multidimensionale, effettuata da un team multidisciplinare i cui attori principali sono il medico geriatria, l'infermiere e l'assistente sociale e si pone tra gli obiettivi quelli di determinare le capacità funzionali residue, descrivere la rete minima assistenziale di servizi socio-sanitari per un assistenza continuativa e creare un Piano di Assistenza Individuale (PAI). Questo presuppone anche una definizione delle diagnosi infermieristiche, le cui più comuni sono:

- Inefficaci prestazioni di ruolo;

- Inefficace mantenimento della salute;

- Rischio di cadute;

- Compromissione della mobilità;

- Insufficienza di attività diversive;

- Deficit nella cura di sé;

- Compromissione delle interazioni sociali;

- Compromissione della deambulazione.

Allo stesso tempo si evince, tramite funzioni compromesse, diagnosi infermieristiche secondo la tassonomia NANDA:

- Declino cognitivo $\rightarrow$ Ansia, rischio di scarsa autostima situazionale, rischio di caduta;

- Riduzione della mobilità e sindrome da immobilizzazione $\rightarrow$ Intolleranza all'attività;

- Rischio di cadute $\rightarrow$ Scarsa autostima situazionale, coping difensivo;
- Polipatologia $\rightarrow$ Ansia, scarsa autostima, compromissione degli scambi gassosi;

- Polifarmacologia $\rightarrow$ Rischio di avvelenamento, conflitto decisionale, coping inefficace;

- Depressione $\rightarrow$ Ansia, Rischio di automutilazione, rischio scarsa autostima;

- Incontinenza urinaria e fecale $\rightarrow$ Rischio scarsa autostima, coping inefficace, deficit nella cura di sé;

- Stipsi $\rightarrow$ Diarrea, disfunzione della motilità intestinale, incontinenza fecale;

- Malnutrizione e disidratazione $\rightarrow$ Fatigue, deficit nella cura di sé, compromissione della deglutizione;

- Morte $\rightarrow$ Dolore acuto, dolore cronico, rischio di sindrome da immobilizzazione, lutto.(262)

- Ridurre il rischio di declino funzionale nell'anziano ospedalizzato può avere un impatto significativo nelle loro funzioni e nella loro qualità di vita. Sono state evidenziate numerose strategie basate sulle evidenze scientifiche al fine di ridurre il deterioramento cognitivo negli anziani ospedalizzati. Questi includono:

- Condurre una valutazione geriatrica completa e interdisciplinare dello stato fisico, psicosociale e funzionale già all'inizio del ricovero;

- Incoraggiare l'attività durante l'ospedalizzazione con esercizi strutturati, con esercizi di resistenza progressiva e con programmi di deambulazione, il tutto in collaborazione con terapia riabilitative (fisiche o occupazionali);

- Implementare la mobilizzazione precoce per i pazienti acuti e critici in base a protocolli prestabiliti;

- L'utilizzo di dispositivi come: apparecchi acustici, occhiali, walker o bastone;

- Garantire l'utilizzo di calzature adatte per incoraggiare la deambulazione e prevenire le cadute;

- Migliorare i fattori ambientali per l'assistenza all'anziano come: corrimani, corridoi aperti, grandi orologi e calendari, alza water e porte con maniglie antipanico;

- Integrare i protocolli al fine di ridurre i rischi per le sindromi geriatriche, migliorare la cura di sé, la continenza, la nutrizione, la mobilità, il sonno, le cure igieniche, l'orientamento e minimizzare gli effetti avversi come cateterismi urinari;

- Valutare l'adeguatezza dei farmaci, ridurre al minimo le terapie sedative, garantendo la corretta dose prescritta, monitorare gli effetti delle terapie farmacologiche e garantire la fornitura dei medicinali sia durante il ricovero che alla dimissione;

- Promuovere l'indipendenza, mantenendo la dignità.(263)

\section{La dimissione difficile}

Negli ultimi decenni la popolazione ha cambiato le sue caratteristiche. L'invecchiamento rappresenta una 
delle prime variabili; infatti oggi l'Italia è uno dei paesi più vecchi d'Europa e del mondo con la sua quota di ultrasessantacinquenni sul totale della popolazione.(264) Segue il concetto di fragilità come stato non specifico di vulnerabilità, che riflette cambiamenti fisiologici multisistemici che non sempre equivalgono ad una diagnosi di malattia. Variabili psicologiche, cognitive e sociali contribuiscono in questa sindrome.(265) Un'altra caratteristica è la cronicità delle patologie. L'OMS definisce malattia cronica come: problemi di salute che richiedono un trattamento continuo durante un periodo di tempo da anni a decadi e fa un chiaro riferimento all'impegno di risorse, umane, gestionali ed economiche, in termini sia di costi diretti (ospedalizzazione, farmaci, assistenza medica ecc.) che indiretti (mortalità prematura, disabilità nel lungo termine, ridotta qualità di vita ecc.), necessarie per il loro controllo.(264) Inoltre questo tipo di popolazione è affetta da più patologie contemporaneamente. Le comorbilità sono serie condizioni mediche che non sono direttamente collegate alla diagnosi primaria, ma possono coinvolgere qualsiasi altro organo maggiore.(266)

Questa serie di nuove caratteristiche contraddistinguono una popolazione complessa ed una nuova sfida per il professionista in sanità che dovrà dimettere e seguire questa tipologia di pazienti.

La dimissione è un processo e non un evento isolato. La sua pianificazione deve essere precoce al fine di creare le condizioni affinché pazienti e familiari siano in grado di contribuire alle migliori decisioni da prendere.(267) In una visone più ampia, presidiare questo processo può permettere di agire sulle altre variabili introdotte prima come costi, riammissioni ospedaliere, tempi di ricovero, mortalità e qualità di vita.(268-270)

\section{Il fine vita}

La fase del fine vita è una situazione di difficile gestione per il personale sanitario. Esso vive infatti la sconfitta del piano di cura e di assistenza: il rischio che si corre è che gli atteggiamenti diventino frettolosi, si entri nella stanza ad occhi bassi, si cerchi di avere il minimo rapporto relazionale con l'assistito e si fugga fuori rapidamente. Non c'è più niente da fare diventa la frase più ricorrente tra il personale di assistenza e le domande $E$ perché?, E perché proprio a $m e$ ? arrivano dall'assistito alla famiglia e all'infermiere e al medico. Il silenzio dell'impotenza diventa imbarazzante e spesso si vive fino in fondo la propria incapacità di assistere e di fare. La situazione è indiscutibilmente complessa, ma il nostro concetto assistenziale deve modificarsi da non c'è più niente da fare a abbiamo tanto da fare per la persona e la famiglia. Diventa fondamentale adottare strategie per migliorare la qualità di vita dell'assistito anche in questa ultima delicata fase della vita.(271)

Negli ultimi decenni, è stata diffusa l'adozione di percorsi di cura di fine vita come uno strumento per gestire meglio la cura dei morenti in una varietà di impostazioni di cura. L'adozione di vari percorsi di assistenza per il fine vita è avvenuta nonostante la mancanza di prove a supporto del loro utilizzo.(272)

La cura degli individui morenti è parte integrante dell'assistenza sanitaria. La prevalenza delle malattie croniche negli anziani, combinata con l'invecchiamento della popolazione, fa sì che l'erogazione di cure di alta qualità, specifiche per i bisogni degli individui nel fine vita e delle loro famiglie diventi un imperativo. A seguito dell'incremento della longevità, infatti, gli individui con condizioni patologiche croniche e limitanti vivranno più a lungo con l'onere della malattia o del declino funzionale. Gli infermieri possono in tal senso, integrare la gestione dei sintomi, il processo decisionale e la cura di supporto nel processo di assistenza primario per migliorare la qualità di vita e ridurre l'impatto economico ed emotivo alla fine della vita.(273-275) Le malattie croniche infatti sono causa di morte per 40 milioni di persone ogni anno (circa il $70 \%$ di tutti i decessi a livello globale). Il numero maggiore di decessi è dovuto alle malattie cardiovascolari (17,7 milioni di persone), seguite dai tumori (8,8 milioni), dalle malattie respiratorie (3,9 milioni) e dal diabete (1,6 milioni). L' $87 \%$ dei decessi avviene nei Paesi a basso-medio reddito.(276) Secondo un recente studio in Italia il $44,4 \%$ della popolazione in fase terminale affetta sia da patologie oncologiche sia da patologie croniche-degenerative, muore a domicilio, il $45,7 \%$ in ospedale, il $6,1 \%$ in Hospice e il $3,8 \%$ in altri contesti (Rsa, ambulanza ecc.). Il 50\% dei decessi avviene nei reparti di medicina generale (Ministero della Salute, 2014).(276)

Le cure palliative sono sia una filosofia che un approccio alla cura. Anche se i termini cure palliative, Hospice e cure fine vita, sono spesso usati come sinonimi, ci sono delle differenze concettuali. Le cure palliative non sono limitate alla cura degli ultimi mesi o giorni di vita. Piuttosto, le cure palliative possono essere usate in combinazione con trattamenti curativi. L'Hospice, invece, fa riferimento agli ultimi mesi di vita e porta con se l'associazione di cure che avvengono in strutture specializzate. Le cure di fine vita, rispecchiano più accuratamente l'accento sugli ultimi giorni e ore di vita.

L'OMS definisce le cure palliative come un approccio alla cura che mira a: Migliorare la qualità di vita dei pazienti e delle loro famiglie affrontando $i$ problemi legati alla malattia potenzialmente mortale, attraverso la prevenzione e il sollievo della sofferenza, per mezzo di un 'identificazione precoce e della valutazione e del trattamento impeccabile del dolore e di altri problemi, fisici, psicosociali e spirituali.

L'articolo 35 del Codice Deontologico dell'infermiere del 2009 definisce che l'infermiere presta assistenza qualunque sia la condizione clinica e fino al termine della vita all'assistito, riconoscendo l'importanza della palliazione e del conforto ambientale, fisico, psicologico, relazionale, spirituale.

All'interno della filosofia delle cure palliative, la 
morte è vista come un processo normale, dunque, lo scopo delle cure palliative non è quello di affrettare nè di ritardare la morte, bensì di supportare il paziente e la famiglia in tutti quegli aspetti citati precedentemente.

Il focus del sistema sanitario sugli approcci curativi ha evidenziato che gli operatori sanitari in effetti non hanno molta familiarità con le cure palliative e le competenze necessarie a fornirle. Inoltre, le cure di fine vita sono spesso associate a setting specializzati, come l'Hospice e le unità di cure palliative. Di conseguenza gli operatori sanitari non possono associare un approccio palliativo alle cure nel loro setting, in una unità per acuti o in una lungo degenza. Eppure la maggior parte delle persone muoiono in ospedale e il numero di persone che muoiono a casa o nelle lungo degenze è in aumento. Solo una piccola percentuale di persone ricevono effettivamente cure di fine vita in contesti specializzati e la maggior parte di queste persone muoiono per forme avanzate di cancro. Sebbene le persone con fragilità e con malattie non oncologiche in fase terminale (life-limiting) potrebbero beneficiare delle cure di fine vita, i sanitari si trovano ad affrontare alcune sfide, quali l'identificazione della fase terminale della malattia e i sintomi che cambiano. Migliorare l'istruzione sui segni della morte imminente e integrare rapidamente la filosofia delle cure palliative nel percorso della malattia, sono le strategie che potrebbero migliorare l'assistenza alle persone che vivono con malattie life-limiting nelle strutture sanitarie.(277)

Nei settori dell'assistenza sanitaria, gli infermieri attraverso continue valutazioni, interventi e analisisviluppano un rapporto terapeutico unico con gli individui. Grazie a questa posizione, gli infermieri sono in grado di garantire la continuità delle cure in modo da rispondere alle esigenze degli individui con malattie life-limiting. Parte integrante del ruolo infermieristico è l'advocacy, cioè avviare e sostenere il decision making, fornire un orientamento, informazioni e collaborazione con altri operatori sanitari. A causa della frequenza delle interazioni con le persone, gli infermieri risultano i primi sanitari a riconoscere che la persona sta raggiungendo gli ultimi giorni e le ultime ore di vita. È in questo momento cruciale che gli infermieri possono dare un contributo significativo alla vita di un individuo vicino alla morte e alla sua famiglia prima, durante e dopo la morte. Fondamentale per le cure di fine vita è la conoscenza dei problemi associati. A complemento di queste conoscenze sono necessarie competenze infermieristiche per gestire efficacemente la situazione dinamica e complessa che si presenta nelle cure di fine vita.(278) Nella cura dei pazienti che vivono un'estrema vulnerabilità come i pazienti al fine della vita, è possibile aiutarli a sentire il calore, alleviare le sofferenze, rispettare l'autonomia e aiutarli a trovare senso e speranza attraverso compiti quotidiani. L'elemento fondamentale di cura è senza dubbio un approccio basato sulla persona piuttosto che sulla malattia.(279)
Le evidenze a sostegno dell'assistenza infermieristica per le persone al fine vita e per i loro familiari sono considerate rispetto al tipo, alla qualità e al livello.

In letteratura sono presenti numerose evidenze che definiscono le competenze attese per gli infermieri nella gestione del paziente nel periodo del fine vita $\mathrm{e}$ forniscono le linee guida per una miglior pratica infermieristica, tenendo comunque in considerazione che tali indicazioni in questo ambito specifico, non devono essere applicate come un libro di cucina, ma utilizzate come strumento per aiutare il processo decisionale nelle cure individuali, oltre ad assicurare che vengano posti in essere strutture e supporti per fornire la migliore assistenza possibile.

\section{Obiettivo}

L'obiettivo generale è quello di: attraverso una revisione di linee guida integrate con overview di revisioni sistematiche e revisione narrativa delle principali prove di efficacia presenti in letteratura, identificare le migliori evidenze disponibili in materia di assistenza infermieristica all'interno di tutti gli ambiti descritti; identificare quindi, attraverso un'analisi delle evidenze, le attività assistenziali e declinarle per livelli di competenza.

L'identificazione di tre livelli di competenza $d i$ base, ottimale e distintiva viene proposta prendendo spunto dalla griglia di valutazione delle competenze del medico internista definita da FADOI (Federazione delle Associazioni Dirigenti Ospedalieri Internisti).

Vengono definite:

- Professionalità di base le competenze specialistiche essenziali considerate minime per l'accesso al lavoro. Necessità di ulteriore training.

- Professionalità ottimali le migliori competenze specialistiche desiderabili nella gestione dei pazienti complessi

- Professionalità distintive le competenze aggiuntive praticate nell'ambito della medicina interna per cui il professionista rappresenta un riferimento ed è competente per formare altri professionisti.(6)

\section{Metodi}

Durante i mesi di Giugno e Luglio 2017, per ogni ambito descritto, è stata condotta una revisione di linee guida integrata con overview delle principali revisioni sistematiche reperibili sulle banche dati Cochrane Library, Medline, Cinahl, Scopus, Embase, Google Scholar e revisione delle risorse afferenti a letteratura primaria più recente.

Le referenze ottenute sono state selezionate da un unico revisore che ha provveduto, una volta identificati gli studi inclusi, ad estrapolare tutte quelle attività assistenziali per cui veniva richiesto l'esercizio di una competenza a qualsiasi livello.

In un secondo tempo, gli ambiti di attività sono 
stati divisi all'interno di tre livelli di professionalità: di base, ottimali e distintive, tenendo conto del grado di professionalità richiesto per esercitare la competenza specifica.

\section{Risultati}

I risultati sono riportati da Tabella 1 a Tabella 27 (280-413).

\section{Conclusioni}

I reparti di Medicina Interna rappresentano di fatto organizzazioni ad elevata complessità, cui afferiscono pazienti tra loro assai differenti per tipologia e con flussi non programmabili (adulti, generalmente anziani o molto anziani con diverse comorbilità; con malattie croniche/cronico-riacutizzate; spesso fragili/ instabili/critici; a volte disabili o a rischio di perdita dell'autosufficienza; limitata speranza di vita; in trattamento polifarmacologico; frequenti ricoveri ripetuti; a rischio di dimissione difficile; che richiedono continuità assistenziale). La complessità dei casi trattati risulta rilevante sia per gravità clinica, fragilità, comorbilità e disabilità, sia per quadri atipici di presentazione. Si riscontrano, di conseguenza, oggettive incertezze e difficoltà nel semplificare i percorsi diagnosticoterapeutici ed assistenziali. In tale contesto, il documento FADOI del 2011 definisce Clinical Competence il risultato delle conoscenze, abilità e capacità tecniche, delle qualità professionali, manageriali, relazionali $\mathrm{e}$ operative di ogni singolo specialista nel contesto sanitario di riferimento. Tale risultato presuppone un bilanciamento ottimale di alcune componenti -sapere, saper fare e saper essere - in una prospettiva di interazione tra professionista sanitario e paziente e decisioni pratiche per la risoluzione di problemi clinici. In questo senso la competenza medica non può essere di per sé garantita da titoli accademici e, al contempo, clinica ed esperienza del medico devono necessariamente essere complementari, poiché non è possibile sostituire l'una con l'altra privilegiando le tecniche di calcolo esplicito, quantitativo e statistico rispetto alla capacità di intuizione e ragionamento clinico. E'necessario quindi che la competenza clinica si fondi su: esperienza, conoscenza scientifica, medicina basata sull'evidenza e buonsenso clinico.(14)
Sempre FADOI, dando per consolidata la fase del curriculum professionale obbligatorio che conduce alla laurea ed al conseguimento di una specializzazione, pone quindi l'accento sul tema del successivo sviluppo professionale, dichiarando che essere, o continuare a essere un buon medico si basa su due caposaldi complementari: l'acquisizione di nuove conoscenze (attraverso l'Educazione Continua in Medicina) e lo sviluppo delle capacità per attuarle in modo competente (che si fonda sull'acquisizione continua di nuove conoscenze, capacità e comportamenti che rendano possibile un competente esercizio della pratica medica).(414)

In questa prospettiva, al fine di valutare le competenze (nell'ottica dell'accreditamento istituzionale, della valutazione professionale, della progressione formativa e del pensiero scientifico), FADOI ha proposto la definizione di schede valutative per il medico internista al fine di graduarne i livelli decisionali di responsabilità e di consapevolezza, stabilendo la segmentazione delle competenze che muovono da una professionalità $d i$ base e una ottimale, ad una eccellente e distintiva, sulla base dei requisiti di maturità professionale richiesti ad ogni livello al professionista.(14)

Sulla scia del lavoro FADOI, considerando quanto già accennato in merito alla competenza indispensabile all'esercizio della professione infermieristica e sulla spinta delle conoscenze attuali, si è ipotizzata una definizione e suddivisione delle competenze richieste all'infermiere di Medicina Interna.

I limiti di questo lavoro sono da rintracciarsi prevelentemente nell'alta esposizione a publication bias che overviews di linee guida di questo tipo possono presentare, ma, soprattutto, alla necessità di integrazione per quelle che rimangono, ancora oggi, le aree inesplorate in cui viene erogata assistenza infermieristica in medicina interna: il paziente ematologico, l'assistenza al paziente in stato di shock, l'assistenza al paziente con patologia renale cronica, ecc.

Le ricadute all'interno della pratica sono tutte da rintracciarsi all'interno di un processo che, tenendo conto dell'evoluzione continua, costante e necessaria della professione, deve necessariamente partire da una ricerca di consenso della declinazione delle competenze qui avvenuta, ricerca di consenso che può rappresentare un punto di partenza per gli sviluppi di ricerca futuri. 
Tabella 1. Nursing clinical competence: il paziente cronico.

\begin{tabular}{|c|c|c|c|}
\hline \multicolumn{4}{|c|}{ ASSISTENZA AL PAZIENTE CRONICO ${ }^{17-24,280-287}$} \\
\hline Ambiti di competenza & Professionalità di base & Professionalità ottimale & Professionalità distintiva \\
\hline \multirow[t]{8}{*}{ Relazione terapeutica } & $\begin{array}{l}\text { Instaurare un contratto educativo } \\
\text { con il paziente basato sulla fiducia, } \\
\text { ascolto attivo ed empatia }\end{array}$ & $\begin{array}{l}\text { Descrivere il profilo psicologico } \\
\text { del paziente }\end{array}$ & $\begin{array}{l}\text { Supervisione del piano di cura } \\
\text { messo in atto dagli operatori sanitari }\end{array}$ \\
\hline & $\begin{array}{l}\text { Essere in grado di riconoscere le fasi } \\
\text { del processo di relazione }\end{array}$ & $\begin{array}{l}\text { Acquisire e mettere in pratica } \\
\text { le tecniche di comunicazione } \\
\text { avanzate } \\
\text { (es. Elicit-Provide-Elicit, } \\
\text { Teach Back, colloquio } \\
\text { motivazionale) }\end{array}$ & $\begin{array}{l}\text { Formare i professionisti sanitari } \\
\text { all'utilizzo di tecniche di } \\
\text { comunicazione avanzate }\end{array}$ \\
\hline & $\begin{array}{l}\text { Riconoscere i bisogni dei pazienti } \\
\text { e valutare le lacune in termini } \\
\text { di bisogni formativi }\end{array}$ & $\begin{array}{l}\text { Declinare nella pratica il } \\
\text { processo di problem solving }\end{array}$ & $\begin{array}{l}\text { Progettare e condurre attività di } \\
\text { formazione e informazione dei } \\
\text { pazienti e delle famiglie }\end{array}$ \\
\hline & $\begin{array}{l}\text { Insegnare al paziente ad approcciarsi } \\
\text { alla patologia (conoscenza, gestione, } \\
\text { aderenza al trattamento, evoluzione) }\end{array}$ & $\begin{array}{l}\text { Sostenere le attività di formazione } \\
\text { e informazione dei pazienti e delle } \\
\text { loro famiglie valorizzando il ruolo } \\
\text { delle Associazioni di tutela dei } \\
\text { malati cronici }\end{array}$ & \\
\hline & $\begin{array}{l}\text { Acquisire e mettere in pratica le } \\
\text { tecniche di comunicazione di base } \\
\text { (es. uso di domande aperte, ribadire } \\
\text { i concetti, comunicazione verbale } \\
\text { e non, differenze culturali, ecc...) }\end{array}$ & & \\
\hline & $\begin{array}{l}\text { Conoscere le fasi del processo di } \\
\text { problem solving }\end{array}$ & & \\
\hline & $\begin{array}{l}\text { Indirizzare gradualmente il paziente } \\
\text { al self care }\end{array}$ & & \\
\hline & $\begin{array}{l}\text { Stabilire obiettivi, attuazione del piano } \\
\text { di cura e valutazione intermedia } \\
\text { e finale del percorso assistenziale }\end{array}$ & & \\
\hline \multirow[t]{8}{*}{ Organizzativo } & $\begin{array}{l}\text { Raccolta dati e compilazione cartella } \\
\text { educativa }\end{array}$ & $\begin{array}{l}\text { Concepire e gestire } \\
\text { (pianificazione, coordinamento } \\
\text { e valutazione) programmi di } \\
\text { educazione terapeutica, costruire } \\
\text { piani individualizzati per } \\
\text { l'autogestione continua }\end{array}$ & $\begin{array}{l}\text { Promuovere, progettare, } \\
\text { implementare e valutare programmi } \\
\text { di educazione terapeutica }\end{array}$ \\
\hline & $\begin{array}{l}\text { Educare i pazienti nell'utilizzo delle } \\
\text { risorse sanitarie, sociali ed } \\
\text { economiche disponibili } \\
\text { terapeutica }\end{array}$ & $\begin{array}{l}\text { Partecipare alla progettazione ed } \\
\text { implementazione di protocolli e } \\
\text { linee guida di educazione }\end{array}$ & $\begin{array}{l}\text { Organizzare corsi di formazione per } \\
\text { operatori sanitari, prepararli a diventare } \\
\text { coordinatori di programmi }\end{array}$ \\
\hline & $\begin{array}{l}\text { Attuare programmi di educazione } \\
\text { terapeutica e piani individualizzati per } \\
\text { l'autogestione continua da parte del } \\
\text { paziente, sotto supervisione }\end{array}$ & $\begin{array}{l}\text { Conoscere ed applicare strumenti } \\
\text { di valutazione dell'apprendimento } \\
\text { di pazienti e caregiver, della } \\
\text { compliance, dell'efficacia } \\
\text { dell'empowerment dell'assistito }\end{array}$ & $\begin{array}{l}\text { Progettare ed implementare protocolli } \\
\text { e linee guida }\end{array}$ \\
\hline & $\begin{array}{l}\text { Mettere in pratica protocolli e linee } \\
\text { guida }\end{array}$ & $\begin{array}{l}\text { Aiutare ad organizzare le attività } \\
\text { di un'unità assistenziale in cui } \\
\text { vengono integrati l'educazione } \\
\text { terapeutica ed il supporto del } \\
\text { paziente }\end{array}$ & $\begin{array}{l}\text { Sviluppare, scegliere, testare ed } \\
\text { applicare strumenti di valutazione } \\
\text { dell'apprendimento di pazienti e } \\
\text { caregiver, della compliance, } \\
\text { dell'efficacia dell'empowerment } \\
\text { dell'assistito }\end{array}$ \\
\hline & $\begin{array}{l}\text { Conoscere i programmi di prevenzione } \\
\text { secondaria e terziaria }\end{array}$ & $\begin{array}{l}\text { Conoscenza e gestione delle } \\
\text { tecnologie } e \text {-Health } \\
\text { (es. telemedicina) }\end{array}$ & $\begin{array}{l}\text { Organizzare e coordinare le attività } \\
\text { di un'unità assistenziale in cui } \\
\text { vengono integrati l'educazione } \\
\text { terapeutica ed il supporto del } \\
\text { paziente }\end{array}$ \\
\hline & & $\begin{array}{l}\text { Partecipare a programmi di } \\
\text { prevenzione secondaria e terziaria }\end{array}$ & $\begin{array}{l}\text { Formare i professionisti sanitari } \\
\text { all'utilizzo di tecnologie } e \text {-Health }\end{array}$ \\
\hline & & & $\begin{array}{l}\text { Realizzare programmi di prevenzione } \\
\text { secondaria e terziaria }\end{array}$ \\
\hline & & & $\begin{array}{l}\text { Promuovere politiche di educazione } \\
\text { terapeutica del paziente a livello } \\
\text { istituzionale }\end{array}$ \\
\hline \multirow[t]{3}{*}{$\begin{array}{l}\text { Formazione del } \\
\text { professionista }\end{array}$} & $\begin{array}{l}\text { Iniziale acquisizione di capacità di } \\
\text { autoconsapevolezza personale e } \\
\text { professionale, conoscenza di sé ed } \\
\text { empatia }\end{array}$ & $\begin{array}{l}\text { Adattare l'approccio professionale } \\
\text { alle caratteristiche individuali } \\
\text { del paziente e della sua malattia }\end{array}$ & $\begin{array}{l}\text { Creazione ed attuazione di progetti } \\
\text { di ricerca }\end{array}$ \\
\hline & $\begin{array}{l}\text { Conoscenza dei processi terapeutici } \\
\text { teorici e pratici, dei bisogni } \\
\text { dell'assistito, delle varie patologie e } \\
\text { dei loro processi diagnostico-teraputici }\end{array}$ & Partecipare a progetti di ricerca & $\begin{array}{l}\text { Svolgere attività di tutoraggio, } \\
\text { supervisione clinica e corsi di } \\
\text { aggiornamento }\end{array}$ \\
\hline & $\begin{array}{l}\text { Frequentare corsi riguardo } \\
\text { all'educazione terapeutica }\end{array}$ & $\begin{array}{l}\text { Valutare periodicamente la propria } \\
\text { performance }\end{array}$ & $\begin{array}{l}\text { Valutare periodicamente e migliorare le } \\
\text { performance didattiche degli operatori } \\
\text { sanitari }\end{array}$ \\
\hline Interprofessionale & $\begin{array}{l}\text { Conoscere il ruolo dei vari } \\
\text { professionisti sanitari nell'ambito } \\
\text { dell'educazione terapeutica e } \\
\text { collaborare con essi }\end{array}$ & $\begin{array}{l}\text { Stabilire le proprie competenze } \\
\text { in relazione a quelle delle altre } \\
\text { figure professionali, in modo da } \\
\text { facilitare la creazione di team } \\
\text { multidisciplinari }\end{array}$ & $\begin{array}{l}\text { Utilizzo di metodi per l'empowerment } \\
\text { del paziente, per formare e informare lui } \\
\text { e tutti gli operatori sanitari, e non, } \\
\text { coinvolti } \\
\text { (es. Stanford model) }\end{array}$ \\
\hline
\end{tabular}


Tabella 2. Nursing clinical competence: scompenso cardiaco.

\begin{tabular}{|c|c|c|}
\hline \multicolumn{3}{|c|}{ ASSISTENZA AL PAZIENTE CON SCOMPENSO CARDIACO ${ }^{25-33,288-299}$} \\
\hline Professionalità di base & Professionalità ottimale & Professionalità distintiva \\
\hline $\begin{array}{l}\text { Conoscere la patologia, principali segni e sintomi } \\
\text { e decorso clinico }\end{array}$ & $\begin{array}{l}\text { Esercitare counselling alla persona con } \\
\text { scompenso cardiaco }\end{array}$ & $\begin{array}{l}\text { Progettare piani di dimissione che tengano conto } \\
\text { di variabili complesse, necessità di prosecuzione } \\
\text { di addestramenti specifici, monitoraggio telefonico, } \\
\text { verifica dell'aderenza terapeutica }\end{array}$ \\
\hline Progettare interventi educativi standard ${ }^{25-33,288-299}$ & $\begin{array}{l}\text { Considerare i fattori psicosociali durante il } \\
\text { processo di assistenza quali qualità della vita, } \\
\text { l'isolamento sociale, la depressione e l'ansia }\end{array}$ & $\begin{array}{l}\text { Collaborare all'interno di progetti di adeguamento } \\
\text { di linee guida e sviluppo di procedure e protocolli } \\
\text { assistenziali }\end{array}$ \\
\hline Coinvolgere la famiglia nei processi di cura & Progettare piani educativi personalizzati & $\begin{array}{l}\text { Promuovere, progettare, implementare e } \\
\text { valutare programmi di educazione terapeutica }\end{array}$ \\
\hline $\begin{array}{l}\text { Valutare edemi, stato della cute, apparato } \\
\text { respiratorio, peso }\end{array}$ & $\begin{array}{l}\text { Concepire e gestire (pianificazione, } \\
\text { coordinamento e valutazione) programmi di } \\
\text { educazione terapeutica, costruire piani } \\
\text { individualizzati per l'autogestione continua }\end{array}$ & $\begin{array}{l}\text { Organizzare corsi di formazione per operatori } \\
\text { sanitari, prepararli a diventare coordinatori di } \\
\text { programmi di educazione terapeutica }\end{array}$ \\
\hline $\begin{array}{l}\text { Indirizzare gradualmente il paziente al } \\
\text { self care }\end{array}$ & $\begin{array}{l}\text { Partecipare alla progettazione ed } \\
\text { implementazione di protocolli e linee guida }\end{array}$ & Progettare ed implementare protocolli e linee guida \\
\hline $\begin{array}{l}\text { Stabilire obiettivi, attuazione del piano di cura e } \\
\text { valutazione intermedia e finale del percorso } \\
\text { assistenziale }\end{array}$ & $\begin{array}{l}\text { Conoscere ed applicare strumenti di } \\
\text { valutazione dell'apprendimento di pazienti e } \\
\text { caregiver, della compliance, dell'efficacia } \\
\text { dell'empowerment dell'assistito }\end{array}$ & $\begin{array}{l}\text { Sviluppare, scegliere, testare ed applicare } \\
\text { strumenti di valutazione dell'apprendimento di } \\
\text { pazienti e caregiver, della compliance, } \\
\text { dell'efficacia dell'empowerment dell'assistito }\end{array}$ \\
\hline $\begin{array}{l}\text { Raccolta dati e compilazione cartella } \\
\text { educativa }\end{array}$ & $\begin{array}{l}\text { Aiutare ad organizzare le attività di un'unità } \\
\text { assistenziale in cui vengono integrati } \\
\text { l'educazione terapeutica ed il supporto } \\
\text { del paziente }\end{array}$ & $\begin{array}{l}\text { Organizzare e coordinare le attività di un'unità } \\
\text { assistenziale in cui vengono integrati } \\
\text { l'educazione terapeutica ed il supporto del paziente }\end{array}$ \\
\hline $\begin{array}{l}\text { Educare i pazienti nell'utilizzo delle risorse } \\
\text { sanitarie, sociali ed economiche disponibili }\end{array}$ & $\begin{array}{l}\text { Conoscenza e gestione delle tecnologie } \\
e \text {-Health (es. telemedicina) }\end{array}$ & $\begin{array}{l}\text { Formare i professionisti sanitari all'utilizzo di } \\
\text { tecnologie } e \text {-Health }\end{array}$ \\
\hline $\begin{array}{l}\text { Attuare programmi di educazione terapeutica } \\
\text { e piani individualizzati per l'autogestione } \\
\text { continua da parte del paziente, sotto } \\
\text { supervisione }\end{array}$ & $\begin{array}{l}\text { Partecipare a programmi di prevenzione } \\
\text { secondaria e terziaria }\end{array}$ & $\begin{array}{l}\text { Realizzare programmi di prevenzione } \\
\text { secondaria e terziaria }\end{array}$ \\
\hline Mettere in pratica protocolli e linee guida & $\begin{array}{l}\text { Adattare l'approccio professionale alle } \\
\text { caratteristiche individuali del paziente e della } \\
\text { sua malattia }\end{array}$ & $\begin{array}{l}\text { Promuovere politiche di educazione terapeutica } \\
\text { del paziente a livello istituzionale }\end{array}$ \\
\hline $\begin{array}{l}\text { Conoscere i programmi di prevenzione } \\
\text { secondaria e terziaria }\end{array}$ & Partecipare a progetti di ricerca & Creazione ed attuazione di progetti di ricerca \\
\hline $\begin{array}{l}\text { Iniziale acquisizione di capacità di } \\
\text { autoconsapevolezza personale e professionale, } \\
\text { conoscenza di sé ed empatia }\end{array}$ & $\begin{array}{l}\text { Valutare periodicamente la propria } \\
\text { performance }\end{array}$ & $\begin{array}{l}\text { Svolgere attività di tutoraggio, supervisione clinica } \\
\text { e corsi di aggiornamento }\end{array}$ \\
\hline $\begin{array}{l}\text { Conoscenza dei processi terapeutici teorici } \\
\text { e pratici, dei bisogni dell'assistito, delle } \\
\text { varie patologie e dei loro processi } \\
\text { diagnostico-teraputici }\end{array}$ & $\begin{array}{l}\text { Stabilire le proprie competenze in relazione } \\
\text { a quelle delle altre figure professionali, in } \\
\text { modo da facilitare la creazione di team } \\
\text { multidisciplinari }\end{array}$ & $\begin{array}{l}\text { Valutare periodicamente e migliorare le } \\
\text { performance didattiche degli operatori sanitari }\end{array}$ \\
\hline $\begin{array}{l}\text { Frequentare corsi riguardo all'educazione } \\
\text { terapeutica }\end{array}$ & & $\begin{array}{l}\text { Utilizzo di metodi per l'empowerment del paziente, } \\
\text { per formare e informare lui e tutti gli operatori sanitari } \\
\text { e non, coinvolti (es. Stanford model) }\end{array}$ \\
\hline $\begin{array}{l}\text { Conoscere il ruolo dei vari professionisti } \\
\text { sanitari nell'ambito dell'educazione } \\
\text { terapeutica e collaborare con essi }\end{array}$ & & \\
\hline
\end{tabular}


Tabella 3. Nursing clinical competence: la broncopneumopatia cronica e ostruttiva.

\begin{tabular}{|c|c|c|}
\hline \multicolumn{3}{|c|}{ ASSISTENZA AL PAZIENTE CON BPCO ${ }^{34-44}$} \\
\hline Professionalità di base & Professionalità ottimale & Professionalità distintiva \\
\hline $\begin{array}{l}\text { Riconoscere le principali alterazioni dei } \\
\text { parametri vitali e delle condizioni cliniche }\end{array}$ & $\begin{array}{l}\text { Educare il paziente e caregiver al } \\
\text { riconoscimento di alterazioni delle } \\
\text { condizioni cliniche }\end{array}$ & Educare il paziente al self-management \\
\hline $\begin{array}{l}\text { Effettuare accurato accertamento } \\
\text { infermieristico seguendo un modello } \\
\text { di anamnesi infermieristica }\end{array}$ & $\begin{array}{l}\text { Valutare il grado di conoscenza delle } \\
\text { complicanze della patologia }\end{array}$ & $\begin{array}{l}\text { Attuare percorso di dimissione protetta (in base } \\
\text { alle caratteristiche specifiche del soggetto) }\end{array}$ \\
\hline Indagare alterazioni riposo-sonno & $\begin{array}{l}\text { Valutare la compliance all'utilizzo di } \\
\text { ossigenoterapia inalatori al domicilio }\end{array}$ & $\begin{array}{l}\text { Pianificare un percorso per il raggiungimento } \\
\text { degli obiettivi condivisi con il paziente durante } \\
\text { il ricovero }\end{array}$ \\
\hline Riconoscere alterazioni nelle ADL & $\begin{array}{l}\text { Verificare la compliance alla terapia } \\
\text { farmacologia }\end{array}$ & $\begin{array}{l}\text { Cooperare con l'équipe per garantire un processo } \\
\text { assistenziale individualizzato }\end{array}$ \\
\hline $\begin{array}{l}\text { Individuare figure di supporto al domicilio } \\
\text { (se presenti) }\end{array}$ & Riconoscere alterazioni nutrizionali/mataboliche & $\begin{array}{l}\text { Promuovere l'auto-cura fornendo consigli e } \\
\text { rafforzando le conoscenze del paziente }\end{array}$ \\
\hline $\begin{array}{l}\text { Informare il paziente sugli interventi di } \\
\text { prevenzione (stop fumo) }\end{array}$ & $\begin{array}{l}\text { Guidare il paziente/caregiver durante il } \\
\text { ricovero su quelli che saranno gli aspetti } \\
\text { clinico-assistenziali }^{34-44}\end{array}$ & $\begin{array}{l}\text { Valutare se il paziente ha realmente compreso } \\
\text { tutte le informazioni fornite }\end{array}$ \\
\hline $\begin{array}{l}\text { Stimolare il paziente a svolgere attività fisica } \\
\text { in base al grado di dispnea rilevato }\end{array}$ & $\begin{array}{l}\text { Valutare la comprensione di atteggiamenti } \\
\text { per migliorare il proprio stato di salute }\end{array}$ & $\begin{array}{l}\text { Trasmettere le proprie conoscenze in modo fruibile } \\
\text { per il paziente }\end{array}$ \\
\hline $\begin{array}{l}\text { Saper utilizzare i principali dispositivi per la } \\
\text { somministrazione di ossigenoterapia }\end{array}$ & $\begin{array}{l}\text { Valutare il grado di comprensione e la gravità } \\
\text { della propria patologia }\end{array}$ & Stimolare l'autonomia della persona \\
\hline $\begin{array}{l}\text { Riconoscere eventuali alterazioni dello stato } \\
\text { di coscienza }\end{array}$ & $\begin{array}{l}\text { Stimolare il paziente allo sviluppo delle } \\
\text { proprie abilità sul controllo dei sintomi } \\
\text { della patologia }\end{array}$ & Programmare controlli/follow-up \\
\hline \multirow[t]{6}{*}{ Saper effettuare un prelievo per emogasanalisi } & $\begin{array}{l}\text { Motivare il paziente al cambiamento delle } \\
\text { proprie abitudini di vita se scorrette }\end{array}$ & $\begin{array}{l}\text { Compiere scelte durante il percorso assistenziale } \\
\text { tenendo conto delle volontà del paziente e nel } \\
\text { rispetto della sua prospettiva di vita }\end{array}$ \\
\hline & $\begin{array}{l}\text { Garantire continuità assistenziale, e supporto } \\
\text { al domicilio (rete assistenziale territoriale) }\end{array}$ & $\begin{array}{l}\text { Organizzare un programma di dimissione pianificato } \\
\text { in prospettiva di un mantenimento/miglioramento } \\
\text { della qualità di vita }\end{array}$ \\
\hline & Educare il paziente alla gestione della dispnea & $\begin{array}{l}\text { Garantire sostegno psicologico e stimolare la persona } \\
\text { ad una vita sociale attiva (in base a quanto la gravità } \\
\text { della patologia lo consenta) }\end{array}$ \\
\hline & $\begin{array}{l}\text { Educare il paziente al corretto utilizzo } \\
\text { dell'ossigeno terapia e degli inalatori }\end{array}$ & $\begin{array}{l}\text { Informare ed educare il paziente su come } \\
\text { programmare le attività di vita quotidiana, sulla } \\
\text { base delle proprie aspettative e preferenze }\end{array}$ \\
\hline & & $\begin{array}{l}\text { Educare il paziente al corretto stile di vita da seguire } \\
\text { (nutrizione, attività fisica, riposo sonno) }\end{array}$ \\
\hline & & $\begin{array}{l}\text { Saper applicare i dispositivi per l'erogazione di } \\
\text { ventilazione meccanica non invasiva }\end{array}$ \\
\hline
\end{tabular}

Tabella 4. Nursing clinical competence: la cirrosi epatica.

\begin{tabular}{|c|c|c|}
\hline \multicolumn{3}{|c|}{ ASSISTENZA AL PAZIENTE CON CIRROSI EPATICA ${ }^{45-53,300-302}$} \\
\hline Professionalità di base & Professionalità ottimale & Professionalità distintiva \\
\hline $\begin{array}{l}\text { Conoscere la fisiopatologia della cirrosi } \\
\text { epatica }\end{array}$ & $\begin{array}{l}\text { Utilizzare scale di valutazione validate per la } \\
\text { stadiazione della cirrosi epatica }\end{array}$ & Considerare i risultati delle indagini diagnostiche \\
\hline $\begin{array}{l}\text { Eseguire un accurato accertamento } \\
\text { infermieristico }\end{array}$ & $\begin{array}{l}\text { Valutare il fabbisogno educativo del paziente } \\
\text { e della famiglia }\end{array}$ & Partecipare a corsi di aggiornamento avanzato \\
\hline Rilevare i principali i fattori di rischio & Valutare la capacità di apprendimento & $\begin{array}{l}\text { Effettuare ricerche in campo scientifico } \\
\text { utilizzando banche dati per incrementare le } \\
\text { proprie conoscenze }\end{array}$ \\
\hline Eseguire un accurato esame obiettivo & $\begin{array}{l}\text { Valutare l'aderenza terapeutica del paziente } \\
\text { (scala Morisky) }\end{array}$ & $\begin{array}{l}\text { Progettare corsi di aggiornamento al fine di } \\
\text { uniformare le conoscenze nella propria equipe }\end{array}$ \\
\hline $\begin{array}{l}\text { Conoscere le complicanze della cirrosi e i fattori } \\
\text { scatenanti delle complicanze }\end{array}$ & $\begin{array}{l}\text { Riconoscere preventivamente le complicanze } \\
\text { potenziali }\end{array}$ & $\begin{array}{l}\text { Collaborare alla stesura di procedure e protocolli } \\
\text { nella propria azienda }\end{array}$ \\
\hline $\begin{array}{l}\text { Richiedere l'intervento dell'infermiere con } \\
\text { professionalità ottimale per la gestione delle } \\
\text { complicanze }\end{array}$ & $\begin{array}{l}\text { Valutare lo stato nutrizionale del paziente } \\
\text { attraverso scale e validate (MNA) e identifica } \\
\text { i pazienti a rischio malnutrizione }\end{array}$ & Diffondere le procedure all'interno dell'equipe \\
\hline $\begin{array}{l}\text { Conoscere i piani assistenziali adatti al } \\
\text { paziente cirrotico }\end{array}$ & $\begin{array}{l}\text { Conoscere le modalità di preparazione } \\
\text { dell'assistito rispetto alle seguenti indagini: } \\
\text { paracentesi evacuativa, elastografia epatica, } \\
\text { biopsia epatica, ecografia dell'addome }\end{array}$ & \\
\hline Attuare un'assistenza standardizzata & $\begin{array}{l}\text { Conoscere il meccanismo d'azione, } \\
\text { i principali effetti collaterali e le caratteristiche } \\
\text { dei farmaci antivirali per l'epatite B e C }\end{array}$ & \\
\hline Saper preparare clisteri medicati & Pianificare un’assistenza personalizzata & \\
\hline $\begin{array}{l}\text { Collaborare con il medico nell'esecuzione } \\
\text { della paracentesi }\end{array}$ & $\begin{array}{l}\text { Valutare il patrimonio venoso attraverso scale } \\
\text { validate }\end{array}$ & \\
\hline & Gestire la continuità assistenziale & \\
\hline
\end{tabular}


Tabella 5. Nursing clinical competence: la pancreatite.

\begin{tabular}{|c|c|c|}
\hline \multicolumn{3}{|c|}{ ASSISTENZA AL PAZIENTE CON PANCREATITE ACUTA ${ }^{54-55,303-316}$} \\
\hline Professionalità di base & Professionalità ottimale & Professionalità distintiva \\
\hline $\begin{array}{l}\text { Intercettare precocemente i parametri vitali } \\
\text { alterati (FC, TC, FR) e gli esami di laboratorio } \\
\text { (WBC, Lipasi) al di fuori del range di normalità }\end{array}$ & $\begin{array}{l}\text { Conoscere tutti i criteri definenti la } \\
\text { pancreatite acuta }\end{array}$ & $\begin{array}{l}\text { Creare percorsi di educazione alla salute ed } \\
\text { interventi educativi volti a prevenire e gestire } \\
\text { la malattia ed eliminare i possibili fattori di rischio }\end{array}$ \\
\hline Somministrare la terapia endovenosa prescritta & $\begin{array}{l}\text { Riconoscere in misura precoce e preventiva i } \\
\text { segni e sintomi definenti la PA, in particolar } \\
\text { modo identificare il livello di severità }\end{array}$ & $\begin{array}{l}\text { Favorire lo sviluppo di un linguaggio comune } \\
\text { sulla patologia tra le differenti figure specialistiche }\end{array}$ \\
\hline $\begin{array}{l}\text { Raccogliere anamnesi personale, familiare } \\
\text { e clinica }\end{array}$ & $\begin{array}{l}\text { Conoscere i protocolli finalizzati alla } \\
\text { stabilizzazione dei parametri vitali del paziente } \\
\text { (Funzionalità respiratoria, equilibrio } \\
\text { idroelettrolitico, gestione del dolore e } \\
\text { mantenimento dell'integrità corporea) }\end{array}$ & $\begin{array}{l}\text { Favorire lo sviluppo di pratiche comuni attraverso } \\
\text { il continuo studio ed approfondimento delle Linee } \\
\text { Guida all'interno di aree sanitarie e geografiche } \\
\text { differenti }\end{array}$ \\
\hline Monitorare la risposta corporea alla terapia iniziale & Prevenire la potenziale comparsa di trombosi & $\begin{array}{l}\text { Creare protocolli di studio e trial clinici al fine } \\
\text { di rendere massimamente standardizzabili i dati } \\
\text { raccolti dai differenti pazienti }\end{array}$ \\
\hline Compilare scale di valutazione prognostica & $\begin{array}{l}\text { Garantire una continuità assistenziale } \\
\text { attraverso la programmazione di follow-up }\end{array}$ & $\begin{array}{l}\text { Utilizzare metodi standardizzati per valutare } \\
\text { l'efficacia della terapia in atto }\end{array}$ \\
\hline \multirow[t]{2}{*}{$\begin{array}{l}\text { Identificazione il grado di dolore attraverso } \\
\text { le specifiche scale di valutazione }\end{array}$} & $\begin{array}{l}\text { Favorire un'alleanza terapeutica con il paziente } \\
\text { al fine di favorire la compliance al trattamento }\end{array}$ & $\begin{array}{l}\text { Implementare la comunicazione e lo scambio } \\
\text { informazionale tra le diverse strutture specialistiche } \\
\text { territoriali al fine di effettuare un corretto invio del } \\
\text { paziente e ridurre il tasso di mortalità dello stesso }\end{array}$ \\
\hline & & $\begin{array}{l}\text { Partecipare a team multidisciplinari per mantenersi } \\
\text { aggiornati ed in formazione continua }\end{array}$ \\
\hline
\end{tabular}

Tabella 6. Nursing clinical competence: il diabete mellito.

\begin{tabular}{lll}
\hline & ASSISTENZA AL PAZIENTE CON DIABETE MELLITO \\
\hline Profess, 317-319 & \multicolumn{1}{c}{ Professionalità ottimale } & Professionalità distintiva \\
\hline $\begin{array}{l}\text { Incoraggiare il paziente a seguire il programma } \\
\text { educativo e aiutarlo nell'autogestione della malattia }\end{array}$ & $\begin{array}{l}\text { Lavorare in collaborazione con le altre istituzioni } \\
\text { di educazione per definire programmi educativi }\end{array}$ & $\begin{array}{l}\text { Essere a conoscenza di tutte le modalità di gestione } \\
\text { dei trattamenti correnti farmacologici e non }\end{array}$ \\
\hline $\begin{array}{l}\text { Fornire materiale educativo che facilitano } \\
\text { l'apprendimento }\end{array}$ & $\begin{array}{l}\text { Gestire una sicura ed efficacia dimissione } \\
\text { attraverso un piano specifico di educazione }\end{array}$ & $\begin{array}{l}\text { Diffondere le nuove conoscenze attraverso corsi } \\
\text { di formazione agli altri operatori sanitari }\end{array}$ \\
\hline $\begin{array}{l}\text { Programmare un monitoraggio del controllo } \\
\text { glicemico mirato }\end{array}$ & $\begin{array}{l}\text { Identificare il bisogno di cambiamento, } \\
\text { generando innovazioni e soluzioni che vanno }\end{array}$ & $\begin{array}{l}\text { Spiegare ed erogare consulenza riguardo le } \\
\text { complicanze del diabete }\end{array}$ \\
\hline $\begin{array}{l}\text { incontro al paziente e al suo stile di vita } \\
\text { della terapia insulinica }\end{array}$ & attraverso piani di cura individualizzati & \\
\hline $\begin{array}{l}\text { Conoscere modalità di prevenzione di } \\
\text { complicanze quali: ipoglicemia, lipodistrofia, } \\
\text { piede diabetico, neuropatia, retinopatia, } \\
\text { dolore, ulcere }\end{array}$ & $\begin{array}{l}\text { Possedere una conoscenza aggiornata dei } \\
\text { nuovi farmaci in commercio }\end{array}$ & $\begin{array}{l}\text { Partecipare allo sviluppo di linee guida o } \\
\text { protocolli da inserire nella pratica clinica }\end{array}$ \\
\hline
\end{tabular}


Tabella 7. Nursing clinical competence: il delirium.

\begin{tabular}{|c|c|c|}
\hline \multicolumn{3}{|c|}{ ASSISTENZA AL PAZIENTE CON DELIRIUM ${ }^{59-64,320,321}$} \\
\hline Professionalità di base & Professionalità ottimale & Professionalità distintiva \\
\hline $\begin{array}{l}\text { Conoscere ed indagare i fattori di rischio di } \\
\text { delirium all'ingresso e individuare eventuali } \\
\text { indicatori presentatisi nell'ultimo periodo }\end{array}$ & $\begin{array}{l}\text { Utilizzare la scala CAM nei pazienti con } \\
\text { indicatori di rischio con cambiamenti } \\
\text { comportamentali nelle ultime ore }\end{array}$ & $\begin{array}{l}\text { Mettere in atto un piano di trattamento personalizzato, } \\
\text { condiviso con un team multidisciplinare e la famiglia, } \\
\text { implementando interventi non farmacologici come } \\
\text { prima scelta per la prevenzione del delirium } \\
\text { (idratazione, nutrizione, mantenimento ritmo } \\
\text { sonno-veglia, controllo dolore, controllo e revisione } \\
\text { farmaci assunti, mobilizzazione, programmi di } \\
\text { stimolazione cognitiva, ripristino orientamento } \\
\text { spazio-temporale, educazione del personale e della } \\
\text { famiglia, coinvolgimento della famiglia nella cura e } \\
\text { nella terapia occupazionale e fisica quotidiana) }\end{array}$ \\
\hline $\begin{array}{l}\text { Sorvegliare i pazienti a rischio per } \\
\text { identificare modificazioni o fluttuazioni } \\
\text { comportamentali }\end{array}$ & $\begin{array}{l}\text { Mettere in atto abilità e mostra comportamenti } \\
\text { di supporto per la cura dei pazienti e } \\
\text { dei caregivers }\end{array}$ & $\begin{array}{l}\text { Discutere all'interno di un team multidisciplinare } \\
\text { le possibili cause che hanno determinato delirium }\end{array}$ \\
\hline $\begin{array}{l}\text { Diffondere il messaggio che la contenzione } \\
\text { è l'ultima scelta per la gestione di questi } \\
\text { pazienti sia fisica che farmacologica che } \\
\text { nelle misure ambientali }\end{array}$ & $\begin{array}{l}\text { Instaurare relazione terapeutica e di cura } \\
\text { centrata su persona e famiglia }\end{array}$ & $\begin{array}{l}\text { Organizzare e condurre momenti formativi per } \\
\text { personale neo assunto, studenti, infermieri ed } \\
\text { operatori sanitari di varie professioni che } \\
\text { lavorano con anziani }\end{array}$ \\
\hline $\begin{array}{l}\text { Mettere in atto prescrizioni terapeutiche } \\
\text { discusse all'interno del team multidisciplinare } \\
\text { nei pazienti con agitazione severa o con sintomi } \\
\text { che interferiscono con il piano di cura }\end{array}$ & $\begin{array}{l}\text { Coinvolgere la famiglia e caregivers } \\
\text { nell'identificazione dei cambiamenti dei } \\
\text { comportamenti del paziente e nel piano di } \\
\text { cura come riferimento per rassicurarlo e } \\
\text { garantirne la sicurezza }\end{array}$ & $\begin{array}{l}\text { Valutare l'efficacia dei programmi educativi nel } \\
\text { portare reale cambiamento nella pratica lavorativa }\end{array}$ \\
\hline $\begin{array}{l}\text { Prendere parte agli incontri educativi per } \\
\text { accrescere conoscenze su delirium e } \\
\text { aumentare le capacità di cura }\end{array}$ & $\begin{array}{l}\text { Adottare misure di prevenzione sui pazienti } \\
\text { a rischio anche se non hanno manifestato } \\
\text { delirium }\end{array}$ & $\begin{array}{l}\text { Educare paziente e famiglia/caregiver utilizzando } \\
\text { un linguaggio culturalmente adeguato, promuovere il } \\
\text { dialogo in maniera tale che sia perfettamente calato } \\
\text { sulla persona e la sua famiglia; decidere per l'eventuale } \\
\text { intervento di un mediatore culturale. Utilizzare video, } \\
\text { volantini, riferimenti internet come supporto } \\
\text { all'educazione }\end{array}$ \\
\hline $\begin{array}{l}\text { Partecipare a training per sviluppare } \\
\text { capacità comunicative per la gestione dei } \\
\text { pazienti con delirium }\end{array}$ & $\begin{array}{l}\text { Partecipare ad incontri formativi di approfondimento } \\
\text { e mantenimento delle conoscenze e abilità per cura } \\
\text { dei pazienti e loro famiglie/caregivers }\end{array}$ & $\begin{array}{l}\text { Considerare il caregiver come persona fragile } \\
\text { che necessita di educazione e supporto ed è } \\
\text { persona fondamentale per la cura del paziente }\end{array}$ \\
\hline
\end{tabular}

Considerare il paziente nella sua totalità, rispettarne autonomia e capacità di prendere decisioni, comunicare verbalmente e non verbalmente mostrando cura e rispetto

Tabella 8. Nursing clinical competence: la depressione.

\begin{tabular}{|c|c|c|}
\hline \multicolumn{3}{|c|}{ ASSISTENZA AL PAZIENTE CON DEPRESSIONE ${ }^{65-70,322-333}$} \\
\hline Professionalità di base & Professionalità ottimale & Professionalità distintiva \\
\hline $\begin{array}{l}\text { Sviluppare un piano assistenziale adeguato: } \\
\text { monitorare e promuovere nutrizione, } \\
\text { eliminazione, sonno, riduzione dolore }\end{array}$ & $\begin{array}{l}\text { Somministrare periodicamente un test per } \\
\text { valutare il grado di depressione } \\
\text { (GDS-SF/PHQ-9) }\end{array}$ & Applicare il modello IMPACT \\
\hline $\begin{array}{l}\text { Valutare i livelli di abilità funzionale e le } \\
\text { disfunzioni cognitive }\end{array}$ & $\begin{array}{l}\text { Conoscere la correlazione tra processi } \\
\text { metabolici o effetti farmacologici con } \\
\text { l'insorgenza di depressione }\end{array}$ & $\begin{array}{l}\text { Utilizzare la Problem Solving Therapy (PST) } \\
\text { come supporto psicologico }\end{array}$ \\
\hline $\begin{array}{l}\text { Potenziare le funzionalità fisiche e lo } \\
\text { sviluppo di attività sociali }\end{array}$ & Saper fornire un adeguato supporto emotivo & $\begin{array}{l}\text { Conoscere l'elevato rischio di overdose che può } \\
\text { insorgere in pazienti trattati con Venlafaxina }\end{array}$ \\
\hline $\begin{array}{l}\text { Monitorare l'autonomia, l'autocontrollo } \\
\text { e il livello di speranza del paziente }\end{array}$ & $\begin{array}{l}\text { Educare sull'importanza dell'aderenza } \\
\text { terapeutica }\end{array}$ & $\begin{array}{l}\text { Sapere che gli antidepressivi triciclici possono } \\
\text { provocare aritmie o ipotensione }\end{array}$ \\
\hline $\begin{array}{l}\text { Saper fornire informazioni sulla patologia } \\
\text { e i principali trattamenti }\end{array}$ & $\begin{array}{l}\text { Educare il caregiver a continuare il processo } \\
\text { di valutazione anche a domicilio }\end{array}$ & $\begin{array}{l}\text { Conoscere la controindicazione del CITALOPRAM } \\
\text { ed ESCITALOPRAM in pazienti con tratto QT } \\
\text { allungato }\end{array}$ \\
\hline $\begin{array}{l}\text { Conoscere indicativamente il tempo } \\
\text { di raggiungimento dei livelli di } \\
\text { concentrazione plasmatica dei farmaci } \\
\text { antidepressivi }\end{array}$ & $\begin{array}{l}\text { Considerare la depressione un fattore di } \\
\text { incremento del rischio di caduta, } \\
\text { di re-ospedalizzazione e di morte nel } \\
\text { paziente anziano }\end{array}$ & $\begin{array}{l}\text { Eseguire periodicamente un ECG in pazienti } \\
\text { cardiopatici in terapia con CITALOPRAM o } \\
\text { ESCITALOPRAM }\end{array}$ \\
\hline $\begin{array}{l}\text { Saper riconoscere quando la severità clinica } \\
\text { può condurre allo sviluppo di depressione }\end{array}$ & Valutare e riconoscere il rischio di suicidio & \\
\hline $\begin{array}{l}\text { Applicare la Geriatric Depression Scale - } \\
\text { Short Form (GDS-SF) come mezzo } \\
\text { valutativo }\end{array}$ & $\begin{array}{l}\text { Fornire ai caregiver e al paziente informazioni } \\
\text { e/o brochure su organizzazioni di volontariato } \\
\text { o di supporto }\end{array}$ & \\
\hline $\begin{array}{l}\text { Far partecipare il paziente nella scelta del } \\
\text { proprio trattamento personalizzato }\end{array}$ & $\begin{array}{l}\text { Raccomandare di proseguire il trattamento con } \\
\text { antridepressivi per tutto il tempo prescritto } \\
\text { anche in caso di remissione }\end{array}$ & \\
\hline $\begin{array}{l}\text { Riconoscere quando è necessario richiedere } \\
\text { la consulenza di uno specialista } \\
\text { (psichiatra o infermiere psichiatrico) }\end{array}$ & & \\
\hline
\end{tabular}


Tabella 9. Nursing clinical competence: il paziente critico.

\begin{tabular}{|c|c|c|}
\hline \multicolumn{3}{|c|}{ ASSISTENZA AL PAZIENTE CRITICO ${ }^{71-81,334-340}$} \\
\hline Professionalità di base & Professionalità ottimale & Professionalità distintiva \\
\hline Eseguire la raccolta dati in modo sistematico & $\begin{array}{l}\text { Conoscere le metodiche di ventilazione } \\
\text { meccanica non invasiva }\end{array}$ & $\begin{array}{l}\text { Effettuare il posizionamento di accessi vascolari } \\
\text { con tecnica invasiva (PICC, Midline) }\end{array}$ \\
\hline $\begin{array}{l}\text { Promuovere la sicurezza del paziente } \\
\text { (conoscere ed applicare le scale di valutazione } \\
\text { presenti all'interno dell'unità operativa) }\end{array}$ & $\begin{array}{l}\text { Riconoscere alterazioni di PA, FC, quantità } \\
\text { diuresi e drenaggi per intercettare emorragie } \\
\text { interne }\end{array}$ & Eseguire ecografia infermieristica \\
\hline $\begin{array}{l}\text { Conoscere e applicare i protocolli aziendali } \\
\text { per utilizzo di tutti i servizi di supporto } \\
\text { specialistici e diagnostico-strumentali }\end{array}$ & $\begin{array}{l}\text { Programmare ed effettuare regolari cure } \\
\text { igieniche del cavo orale con particolare } \\
\text { attenzione ai pazienti ventilati }\end{array}$ & Organizzare una dimissione protetta/difficile \\
\hline $\begin{array}{l}\text { Pianificare gli interventi di assistenza } \\
\text { infermieristica a seconda del modello } \\
\text { utilizzato all'interno dell'unità operativa }\end{array}$ & Rilevare la pressione venosa centrale & $\begin{array}{l}\text { Controllare in modo mirato e prevenire la comparsa } \\
\text { di delirium }\end{array}$ \\
\hline $\begin{array}{l}\text { Utilizzare i sistemi di monitoraggio dei } \\
\left.\text { parametri vitali di base (PA, FC, TC, } \mathrm{SpO}_{2}\right)\end{array}$ & $\begin{array}{l}\text { Conoscere i principali strumenti di valutazione } \\
\text { della criticità clinica (NEWS, MEWS..) }\end{array}$ & $\begin{array}{l}\text { Saper posizionare una maschera laringea, conoscere } \\
\text { e preparare il materiale per l'intubazione }\end{array}$ \\
\hline $\begin{array}{l}\text { Provvedere al posizionamento di uno o più } \\
\text { accessi venosi garantendo una corretta } \\
\text { somministrazione della terapia farmacologica } \\
\text { endovenosa e non }\end{array}$ & $\begin{array}{l}\text { Riconoscere le principali alterazioni del ritmo } \\
\text { cardiaco }\end{array}$ & $\begin{array}{l}\text { Alla dimissione/trasferimento redarre una scheda } \\
\text { dettagliata contenente: sintesi del ricovero, piano di } \\
\text { eventuale monitoraggio, terapia, piano nutrizionale, } \\
\text { stato infettivo e rischio di sepsi, riabilitazione motoria } \\
\text { e bisogni comunicativi }\end{array}$ \\
\hline $\begin{array}{l}\text { Garantire la pervietà delle vie aeree mediante } \\
\text { manovre di aspirazione o tracheoaspirazione }\end{array}$ & $\begin{array}{l}\text { Effettuare calcolo del p/F dopo esecuzione } \\
\text { di emogasanalisi }\end{array}$ & Conoscere i protocolli ALS \\
\hline Conoscere e applicare le manovre di BLSD & Conoscere i principi di ecografia infermieristica & \\
\hline $\begin{array}{l}\text { Utilizzare la scala AVPU per la valutazione } \\
\text { dello stato di coscienza }\end{array}$ & $\begin{array}{l}\text { Preparare ed accompagnare nel trasporto } \\
\text { intraospedaliero ed extraospedaliero }\end{array}$ & \\
\hline $\begin{array}{l}\text { Controllare e prevenire lesioni correlate } \\
\text { all'immobilità }\end{array}$ & Contribuire alla gestione delle dimissioni protette & \\
\hline Gestire ossigeno terapia in base alla prescrizione & $\begin{array}{l}\text { Impostare un monitoraggio multiparametrico } \\
\text { (PA, FC, TC, } \mathrm{SpO}_{2} \text {, stato di coscienza e dolore) }\end{array}$ & \\
\hline Osservare e ricercare emorragie evidenti & $\begin{array}{l}\text { Utilizzare la scala GCS per la valutazione } \\
\text { dello stato di coscienza }\end{array}$ & \\
\hline $\begin{array}{l}\text { Attivare il sistema di emergenza utilizzando } \\
\text { i sistemi di allertamento precoce e collaborare } \\
\text { alla gestione del paziente in periarresto/arresto } \\
\text { anticipando le prime manovre }\end{array}$ & $\begin{array}{l}\text { Riconoscere e valutare il grado di dipendenza } \\
\text { e di immobilità alterati da un eventuale sedazione } \\
\text { ed applicare misure volte alla prevenzione } \\
\text { delle cadute }\end{array}$ & \\
\hline Eseguire prelievo per emogasanalisi arteriosa & $\begin{array}{l}\text { Gestire NIV e CPAP e il weaning respiratorio } \\
\text { secondo prescrizione medica }\end{array}$ & \\
\hline $\begin{array}{l}\text { Gestire la somministrazione di NPT e NE } \\
\text { da SNG o da PEG secondo prescrizione o } \\
\text { protocolli condivisi }\end{array}$ & $\begin{array}{l}\text { Impostare un monitoraggio diuresi oraria } \\
\text { e calcolare il bilancio idrico }\end{array}$ & \\
\hline $\begin{array}{l}\text { Monitorare e gestire il dolore secondo } \\
\text { prescrizione medica }\end{array}$ & $\begin{array}{l}\text { Monitorare la somministrazione di farmaci } \\
\text { vasoattivi per via endovenosa secondo } \\
\text { prescrizione medica }\end{array}$ & \\
\hline $\begin{array}{l}\text { Pianificare l'assistenza infermieristica } \\
\text { con particolare attenzione alla priorità } \\
\text { degli interventi }\end{array}$ & $\begin{array}{l}\text { Raccogliere con il medico e l'equipe di cura } \\
\text { eventuali direttive sul fine vita }\end{array}$ & \\
\hline Rilevare ed osservare FR e qualità del respiro & 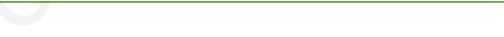 & \\
\hline
\end{tabular}


Tabella 10. Nursing clinical competence: l'ipertensione.

\begin{tabular}{|c|c|c|}
\hline \multicolumn{3}{|c|}{ ASSISTENZA AL PAZIENTE CON IPERTENSIONE ${ }^{82,341-346}$} \\
\hline Professionalità di base & Professionalità ottimale & Professionalità distintiva \\
\hline $\begin{array}{l}\text { Ricercare i fattori anamnestici familiari e } \\
\text { personali, (rivolti alla valutazione dei fattori } \\
\text { di rischio e delle patologie concomitanti), } \\
\text { individuare la figura del caregiver }\end{array}$ & $\begin{array}{l}\text { Effettuare test strumentali specifici (come } \\
\text { ECG, Holter pressorio, etc.) per la ricerca } \\
\text { e la valutazione di eventuali sintomi evocativi } \\
\text { di danno d'organo }\end{array}$ & $\begin{array}{l}\text { Organizzare, gestire, coordinare e partecipare, } \\
\text { a formazioni professionali specifiche in materia }\end{array}$ \\
\hline $\begin{array}{l}\text { Effettuare valutazioni strumentali (P.A., parametri } \\
\text { vitali, peso corporeo, BMI) }\end{array}$ & $\begin{array}{l}\text { Possedere una conoscenza appropriata dei } \\
\text { farmaci in commercio (conoscere eventuali } \\
\text { controindicazioni e combinazioni terapeutiche) }\end{array}$ & $\begin{array}{l}\text { Dimostrare capacità di autonomia nel cercare le } \\
\text { informazioni necessarie per risolvere i problemi } \\
\text { relativi alla pratica professionale }\end{array}$ \\
\hline $\begin{array}{l}\text { Gestire i dati raccolti in cartella e verificare } \\
\text { l'aderenza del paziente al percorso }\end{array}$ & $\begin{array}{l}\text { Conoscere e applicare linee guida e protocolli } \\
\text { ospedalieri dedicati al paziente iperteso }\end{array}$ & $\begin{array}{l}\text { Collaborare con il team di cura per concordare } \\
\text { modalità operative e realizzare l'applicazione e } \\
\text { lo sviluppo di protocolli e linee guida }\end{array}$ \\
\hline $\begin{array}{l}\text { Saper gestire la somministrazione corretta } \\
\text { della terapia }\end{array}$ & $\begin{array}{l}\text { Gestire il piano di dimissione del ricovero } \\
\text { attraverso un piano di continuità assistenziale }\end{array}$ & $\begin{array}{l}\text { Mettere a disposizione le proprie conoscenze teoriche } \\
\text { e pratiche, per interventi informativi ed educativi } \\
\text { specifici rivolti al team interdisciplinare, ai singoli } \\
\text { utenti, a famiglie, gruppi ed altri professionisti } \\
\text { della salute }\end{array}$ \\
\hline $\begin{array}{l}\text { Verificare le conoscenze del paziente } \\
\text { in merito alla propria patologia }\end{array}$ & $\begin{array}{l}\text { Identificare i casi in cui richiedere la consulenza } \\
\text { di altre figure professionali in base alle condizioni } \\
\text { cliniche del paziente, come: dietista etc. }\end{array}$ & $\begin{array}{l}\text { Valutare i progressi delle cure in collaborazione } \\
\text { con il team interdisciplinare }\end{array}$ \\
\hline $\begin{array}{l}\text { Effettuare educazione alla salute sugli stili } \\
\text { di vita corretti (attività fisica, fumo, alcool, } \\
\text { stato nutrizionale) }\end{array}$ & $\begin{array}{l}\text { Spiegare al paziente i possibili effetti collaterali, } \\
\text { istruirlo a consultare il medico per i farmaci } \\
\text { alternativi qualora si verifichino alcuni di questi } \\
\text { effetti collaterali }\end{array}$ & $\begin{array}{l}\text { Gestire le varie attività che sono richieste per } \\
\text { erogare l'assistenza infermieristica ai pazienti in } \\
\text { diversi contesti di cura sia ospedalieri che } \\
\text { territoriali e residenziali }\end{array}$ \\
\hline \multicolumn{3}{|l|}{$\begin{array}{l}\text { Eseguire gli accertamenti di follow-up } \\
\text { (monitoraggio dei comportamenti alimentari, } \\
\text { aderenza allo schema nutrizionale, aderenza } \\
\text { al regime terapeutico) }\end{array}$} \\
\hline $\begin{array}{l}\text { Favorire il patient empowerment ad ogni } \\
\text { incontro, per l'autogestione della malattia. } \\
\text { Istruirlo al controllo della pressione arteriosa } \\
\text { e a tenere un'accurata registrazione dei risultati }\end{array}$ & & \\
\hline
\end{tabular}


Tabella 11. Nursing clinical competence: la trombosi venosa profonda.

\begin{tabular}{|c|c|c|}
\hline \multicolumn{3}{|c|}{ ASSISTENZA AL PAZIENTE CON TROMBOSI VENOSA PROFONDA ${ }^{83-97,347}$} \\
\hline Professionalità di base & Professionalità ottimale & Professionalità distintiva \\
\hline $\begin{array}{l}\text { Conoscere e riconoscere segni e sintomi di } \\
\text { trombosi venosa prodonda, i test diagnostici } \\
\text { principali, i trattamenti farmacologici e } \\
\text { meccanici ed i follow-up }\end{array}$ & $\begin{array}{l}\text { Conoscere segni e sintomi della Sindrome } \\
\text { Post Trombotica (SPT), avvalendosi anche } \\
\text { dell'utilizzo della Villalta Scale Assessment } \\
\text { Symptoms and Clinical Signs Components }\end{array}$ & $\begin{array}{l}\text { Creare momenti di formazione per i colleghi più } \\
\text { giovani e gli studenti in cui spiegare segni e sintomi } \\
\text { di trombosi venosa profonda, includendo diagrammi } \\
\text { od immagini dell'aspetto tipico di un arto affetto da } \\
\text { trombosi venosa profonda }\end{array}$ \\
\hline $\begin{array}{l}\text { Conoscere i fattori di rischio di trombosi venosa } \\
\text { profonda per identificare precocemente coloro che } \\
\text { hanno un rischio aumentato }\end{array}$ & $\begin{array}{l}\text { Applicare il Padua Prediction Score per } \\
\text { valutare il rischio di trombosi }\end{array}$ & $\begin{array}{l}\text { Fornire raccomandazioni per la riduzione del rischio } \\
\text { cardiovascolare }\end{array}$ \\
\hline $\begin{array}{l}\text { Conoscere e riconoscere } \mathrm{i} \text { fattori di rischio correlati } \\
\text { alle terapie anticoagulanti (es. l'efficacia degli } \\
\text { antagonisti della vitamina K può essere influenzata } \\
\text { da diversi fattori come i cambiamenti dietetici, } \\
\text { l'assunzione di alcool o altri medicinali, } \\
\text { la diarrea ed il vomito) }\end{array}$ & $\begin{array}{l}\text { Prevenire la SPT attraverso: utilizzo delle } \\
\text { calze elastiche; deambulazione precoce; } \\
\text { utilizzo degli anticoagulanti; aderenza } \\
\text { terapeutica }\end{array}$ & $\begin{array}{l}\text { Informare il Medico di Medicina Generale dei } \\
\text { trattamenti e dei provvedimenti presi durante il } \\
\text { ricovero che saranno poi da gestire al domicilio }\end{array}$ \\
\hline $\begin{array}{l}\text { Promuovere ed incoraggiare la } \\
\text { deambulazione precoce }\end{array}$ & $\begin{array}{l}\text { Favorire l'assunzione di liquidi a meno che } \\
\text { non ci sia un indicazione clinica che richiede } \\
\text { una restrizione idrica }\end{array}$ & $\begin{array}{l}\text { Prendere in considerazione le preferenze e le } \\
\text { necessità del paziente, concordare un piano di } \\
\text { trattamento; ascoltare le necessità del paziente } \\
\text { ed essere proattivi nella ricerca di soluzioni che } \\
\text { siano su misura }\end{array}$ \\
\hline $\begin{array}{l}\text { Suggerire l'utilizzo della trombo profilassi } \\
\text { meccanica con calze elastiche a compressione } \\
\text { graduata o la compressione pneumatica } \\
\text { intermittente per i pazienti con un elevato } \\
\text { rischio di trombosi o con un elevato rischio di } \\
\text { sanguinamento }\end{array}$ & Applicare i dispositivi meccanici di compressione & $\begin{array}{l}\text { Creare brochure informative così da avere la } \\
\text { possibilità di fare domande ed essere maggiormente } \\
\text { coinvolti nel processo decisionale }\end{array}$ \\
\hline $\begin{array}{l}\text { Conoscere rischi e dei benefici dell'utilizzo } \\
\text { della profilassi meccanica }\end{array}$ & $\begin{array}{l}\text { Conoscere le controindicazioni all'utilizzo } \\
\text { di calze antitrombotiche }\end{array}$ & \\
\hline $\begin{array}{l}\text { Nei pazienti a rischio effettuare una } \\
\text { misurazione quotidiana della circonferenza } \\
\text { degli arti }\end{array}$ & $\begin{array}{l}\text { Avviare un programma di educazione sanitaria } \\
\text { circa il corretto utilizzo dei presidi in cui: porre } \\
\text { attenzione che la calza non venga ripiegata sull'arto; } \\
\text { rimuovere quotidianamente il presidio per scopi } \\
\text { igienici e per verificare la condizione della cute; } \\
\text { nei pazienti con una riduzione significativa della } \\
\text { mobilità, integrità cutanea ridotta o qualsiasi } \\
\text { perdita sensoriale, è necessario ispezionare la pelle } \\
\text { due o tre volte al giorno, in particolare nelle zone } \\
\text { dei talloni e sulle prominenze ossee }\end{array}$ & \\
\hline $\begin{array}{l}\text { Per i pazienti a rischio di trombosi venosa } \\
\text { profonda implementare interventi preventivi, } \\
\text { considerando i fattori di rischio del singolo } \\
\text { e rivalutando regolarmente il piano di cura } \\
\text { del paziente }\end{array}$ & $\begin{array}{l}\text { Insegnare al paziente delle nozioni riguardanti } \\
\text { le posture. In caso di immobilità per lunghi periodi } \\
\text { di tempo, come durante il riposo o durante un lungo } \\
\text { viaggio, è necessario: svegliarsi e camminare ogni } \\
\text { due o tre ore; esercitare le gambe mentre si è seduti; } \\
\text { stringere e rilasciare i muscoli delle gambe; } \\
\text { indossare abiti larghi }\end{array}$ & \\
\hline $\begin{array}{l}\text { Incoraggiare i pazienti all'utilizzo dei } \\
\text { dispositivi di profilassi meccanica e } \\
\text { all'aderenza terapeutica }\end{array}$ & $\begin{array}{l}\text { Addestrare alla somministrazione di terapia } \\
\text { iniettiva sottocutanea, come EBPM o } \\
\text { fundaparinux, il paziente e/o al caregiver }\end{array}$ & \\
\hline
\end{tabular}


Tabella 12. Nursing clinical competence: l'ischemia cerebrale.

\begin{tabular}{|c|c|c|}
\hline \multicolumn{3}{|c|}{ ASSISTENZA AL PAZIENTE CON ISCHEMIA CEREBRALE ${ }^{98-104,348-360}$} \\
\hline Professionalità di base & Professionalità ottimale & Professionalità distintiva \\
\hline $\begin{array}{l}\text { Applicare un monitoraggio parametrico } \\
\text { nella fase acuta e riconoscimento delle } \\
\text { alterazioni da gestire con l'equipe }\end{array}$ & $\begin{array}{l}\text { Promuovere l'integrazione nell'equipe } \\
\text { multidisciplinare con obiettivi comuni }\end{array}$ & $\begin{array}{l}\text { Possedere elevate capacità di supporto, dialogo } \\
\text { e counseling con paziente, famigliari e caregiver } \\
\text { nelle varie fasi della malattia }\end{array}$ \\
\hline $\begin{array}{l}\text { Gestire i bisogni assistenziali principali } \\
\text { nelle varie fasi }\end{array}$ & $\begin{array}{l}\text { Programmare e condurre piani assistenziali } \\
\text { completi del paziente nella fase acuta, } \\
\text { post acuta e cronica-riabilitativa con } \\
\text { attenzione ai bisogni e alle possibili } \\
\text { complicanze da riconoscere e trattare } \\
\text { in equipe }\end{array}$ & $\begin{array}{l}\text { Condurre processi educativi a paziente e caregiver } \\
\text { circa i comportamenti e adeguamenti da adottare per } \\
\text { mantenere comunicazione e deglutizione funzionali, } \\
\text { mobilizzazione efficace, gestione dei bisogni efficiente, } \\
\text { limitazione del rischio di caduta/LdP/TVP/EP } \\
\text { e inserimento nel piano di cura il più precocemente } \\
\text { possibile, anche in vista del rientro al domicilio }\end{array}$ \\
\hline Valutare presenza e tipologia della disfagia & $\begin{array}{l}\text { Riconoscere precocemente e programmare } \\
\text { prevenzione e gestione delle complicanze } \\
\text { legate alla sindrome da allettamento } \\
\text { e all'alterazione dello stato neurologico }\end{array}$ & $\begin{array}{l}\text { Conoscere i principali protocolli relativi alle } \\
\text { procedure di trombolisi farmacologica }\end{array}$ \\
\hline $\begin{array}{l}\text { Valutare il rischio di complicanze legate alla } \\
\text { sindrome da allettamento e all'alterazione } \\
\text { dello stato neurologico e loro prevenzione }\end{array}$ & $\begin{array}{l}\text { Attuare interventi che mantengano la } \\
\text { comunicazione funzionale }\end{array}$ & $\begin{array}{l}\text { Mantenere un aggiornamento costante e capacità } \\
\text { di applicazione da parte dell'equipe delle nuove } \\
\text { evidenze assistenziali, delle schede di valutazione } \\
\text { specifiche dello stato neurologico, del rischio di } \\
\text { complicanze e di capacità funzionale }\end{array}$ \\
\hline $\begin{array}{l}\text { Riconoscere, classificare e gestire } \\
\text { l'incontinenza urinaria }\end{array}$ & $\begin{array}{l}\text { Gestire la disfagia con interventi specifici } \\
\text { per il mantenimento di deglutizione efficace } \\
\text { o priva di complicanze nei vari contesti di malattia; } \\
\text { promuovere la collaborazione multidisciplinare } \\
\text { qualora gli interventi valutativi e adattativi primari } \\
\text { non risultino sufficienti }\end{array}$ & \\
\hline $\begin{array}{l}\text { Valutare la capacità di esecuzione di ADL e } \\
\text { iADL, programmare supporto e compensazione } \\
\text { dove vi sia necessità }\end{array}$ & $\begin{array}{l}\text { Riconoscere, gestire e monitorare la ritenzione } \\
\text { urinaria con i presidi avanzati }\end{array}$ & \\
\hline Valutare e prevenire il rischio di caduta & & \\
\hline $\begin{array}{l}\text { Gestire la postura e la mobilizzazione secondo } \\
\text { la fase clinica, gli obiettivi proposti e la prevenzione } \\
\text { delle complicanze }\end{array}$ & & \\
\hline $\begin{array}{l}\text { Somministrare la terapia specifica prescritta, } \\
\text { con capacità di valutazione del modo di } \\
\text { somministrazione a seconda dei vari contesti } \\
\text { clinici e patologici }\end{array}$ & & \\
\hline
\end{tabular}


Tabella 13. Nursing clinical competence: la disfagia.

\begin{tabular}{|c|c|c|}
\hline \multicolumn{3}{|c|}{ ASSISTENZA AL PAZIENTE CON DISFAGIA ${ }^{108-118}$} \\
\hline Professionalità di base & Professionalità ottimale & Professionalità distintiva \\
\hline $\begin{array}{l}\text { Individuare i pazienti considerati a rischio disfagia } \\
\text { e assicurarsi che venga mantenuta } \\
\text { la prescrizione nulla per bocca }\end{array}$ & $\begin{array}{l}\text { Effettuare uno screening deglutitorio tramite } \\
\text { il Bedside Swallowing Assessment (BSA) } \\
\text { a tutti pazienti considerati a rischio, per } \\
\text { prevenire le complicanze polmonari }\end{array}$ & $\begin{array}{l}\text { Valutare la sintomatologia riferita dal paziente durante } \\
\text { l'atto deglutitorio oggettivandola tramite questionari } \\
\text { self-report (Eat } 10 \text { o il Sydney Swallowing } \\
\text { Questionnaire) }\end{array}$ \\
\hline $\begin{array}{l}\text { Coinvolgere attivamente il paziente e } \mathrm{i} \\
\text { caregivers in tutte le tappe del piano assistenziale } \\
\text { nonché del processo decisionale }\end{array}$ & $\begin{array}{l}\text { Interpretare i risultati dello screening e } \\
\text { classificare la disfagia per gravità: assente, } \\
\text { lieve, moderata e severa }\end{array}$ & $\begin{array}{l}\text { Valutare la possibilità e la fattibilità del fornire } \\
\text { l'indicazione free water nei pazienti con disfagia lieve } \\
\text { e scarsa compliance per i liquidi a consistenza } \\
\text { modificata }\end{array}$ \\
\hline $\begin{array}{l}\text { Somministrare il cibo e i liquidi con la consistenza } \\
\text { adatta alle abilità deglutitore del paziente. } \\
\text { La consistenza dei liquidi deve essere aumentata } \\
\text { utilizzando gli addensanti mentre per gli alimenti } \\
\text { la consistenza da garantire va dalla purea, tritata } \\
\text { sino alla morbida }\end{array}$ & $\begin{array}{l}\text { Assicurare la somministrazione sicura dei } \\
\text { farmaci evitando la frantumazione e andando } \\
\text { a consultare l'elenco dei farmaci do not crush, } \\
\text { che oltre a segnalare i farmaci per i quali non è } \\
\text { possibile praticare la triturazione elencano le } \\
\text { formulazioni alternative }\end{array}$ & $\begin{array}{l}\text { Riconoscere l'eziopatogenesi della sintomatologia } \\
\text { disfagica e mettere in pratica gli aggiustamenti } \\
\text { posturali specialistici (flessione laterale verso il lato } \\
\text { sano, rotazione verso il lato leso, estensione e decubito } \\
\text { laterale) }\end{array}$ \\
\hline $\begin{array}{l}\text { Monitorare il paziente durante i pasti, } \\
\text { riconoscere i segni e sintomi della disfagia } \\
\text { e dell'aspirazione tracheo-bronchiale }\end{array}$ & $\begin{array}{l}\text { Rivalutare le capacità deglutitorie con } \\
\text { cadenza periodica ed a ogni cambiamento } \\
\text { delle condizioni neurologiche, cliniche } \\
\text { e dello stato di deglutizione }\end{array}$ & $\begin{array}{l}\text { Far eseguire le manovre deglutitore facilitatorie in base } \\
\text { al livello di compliance e le capacità residue } \\
\text { del paziente (doppia deglutizione, manovra di } \\
\text { Mendelsohn, deglutizione forzata, sopraglottica } \\
\text { e super-sopraglottica) }\end{array}$ \\
\hline $\begin{array}{l}\text { Osservare e favorire, in base al grado di abilità } \\
\text { e di collaborazione del paziente, } \\
\text { l'igiene orale quotidiana }\end{array}$ & $\begin{array}{l}\text { Fornire istruzione sulle prescrizioni alimentari, } \\
\text { sulle misure di sicurezza da mantenere durante e } \\
\text { dopo la somministrazione del pasto }\end{array}$ & $\begin{array}{l}\text { Eseguire uno screening nutrizionale utilizzando } \\
\text { il Malinutrition Universal Screening Tool (MUST) o il } \\
\text { Nutritional Risk Screening (NRS) }\end{array}$ \\
\hline $\begin{array}{l}\text { Mettere in atto gli aggiustamenti posturali a } \\
\text { carico del capo, collo e tronco (posizione } \\
\text { seduta a } 90^{\circ} \text { con i segmenti corporei sostenuti } \\
\text { e bene allineati, il capo flesso in avanti e il mento } \\
\text { quasi a toccare il manubrio sternale) }\end{array}$ & $\begin{array}{l}\text { Fornire istruzione sulla corretta metodica } \\
\text { di somministrazione dei farmaci }\end{array}$ & \\
\hline $\begin{array}{l}\text { Collaborare alla fase di screening nutrizionale, } \\
\text { da eseguire entro } 24-48 \text { ore dal ricovero in } \\
\text { reparto di modo da prevenire le complicanze } \\
\text { della disidratazione e della malnutrizione }\end{array}$ & $\begin{array}{l}\text { Spiegare, sulla base di quanto prescritto dal } \\
\text { medico specialista, il programma di follow-up } \\
\text { stabilito per l'assistito }\end{array}$ & \\
\hline $\begin{array}{l}\text { Gestire le urgenze respiratorie tramite il } \\
\text { corretto utilizzo dell'aspiratore e le manovre } \\
\text { di disostruzione delle vie aeree in urgenza }\end{array}$ & & \\
\hline $\begin{array}{l}\text { Garantire la somministrazione della terapia } \\
\text { nutrizionale artificiale tramite SNG o PEG, } \\
\text { e prevenire le complicanze annesse alle due } \\
\text { modalità di somministrazione }\end{array}$ & & \\
\hline
\end{tabular}

Tabella 14. Nursing clinical competence: gli squilibri indroelettrici.

\begin{tabular}{lll}
\hline \multicolumn{2}{c}{ ASSISTENZA AL PAZIENTE CON SQUILIBRI IDROELETTROLITICI ${ }^{120-127}$} \\
\hline $\begin{array}{l}\text { Professionalità di base } \\
\text { Ricercare segni e sintomi indicatori dei principali } \\
\text { squilibri elettrolitici (ipo/ipernatriemia, ...) }\end{array}$ & $\begin{array}{l}\text { Conoscere come la quantità di sodio muta } \\
\text { nelle diverse soluzioni infusionali }\end{array}$ & $\begin{array}{l}\text { Professionalità distintiva } \\
\text { ipo/iperpotassiemia }\end{array}$ \\
\hline $\begin{array}{l}\text { Posizionare un accesso venoso periferico } \\
\text { se indicato }\end{array}$ & $\begin{array}{l}\text { Effettuare valuatazioni seriate ed oggettive } \\
\text { dello stato mentale }\end{array}$ & $\begin{array}{l}\text { Riconoscere i segni ECG-grafici di ipo/iperpotassiemia, } \\
\text { in funzione ai livelli di kaliemia }\end{array}$ \\
\hline Effettuare un bilancio idroelettrolitico & $\begin{array}{l}\text { Conoscere le modalità di presentazione } \\
\text { clinica dell'iponatremia, in funzione dei } \\
\text { valori di sodiemia (mEq/L) e in funzione } \\
\text { della velocità di insorgenza dell'iponatremia }\end{array}$ & $\begin{array}{l}\text { Conoscere le manifestazioni cardiovascolari e } \\
\text { neurologiche dell'ipomagnesemia }\end{array}$ \\
\hline $\begin{array}{l}\text { Conoscere gli alimenti in grado di influenzare } \\
\text { la potassiemia (alimenti ricchi di potassio) }\end{array}$ & $\begin{array}{l}\text { Saper valutare i tempi di infusione e la velocità } \\
\text { di correzione }\end{array}$ & $\begin{array}{l}\text { Riconoscere i segni ECG-grafici di } \\
\text { ipermagnesemia }\end{array}$ \\
\hline $\begin{array}{l}\text { Gestire correttamente la terapia infusiva } \\
\text { (compatibilità tra farmaci) e le possibili } \\
\text { complicanze }\end{array}$ & $\begin{array}{l}\text { Ricercare la causa sottostante l'ipernatriemia } \\
\text { e progettare processari educativi volti a prevenirla }\end{array}$ & $\begin{array}{l}\text { Riconoscere le manifestazioni neurologiche e } \\
\text { cardiopolmonari dell'ipofosforemia }\end{array}$ \\
\hline & $\begin{array}{l}\text { Progettare piani educativi rivolti al paziente } \\
\text { per una corretta alimentazione }\end{array}$ & \\
\hline & $\begin{array}{l}\text { Progettare e condurre piani educativi al } \\
\text { paziente per un corretto utilizzo della terapia } \\
\text { con resine chelanti }\end{array}$ & \\
\hline
\end{tabular}


Tabella 15. Nursing clinical competence: le polmoniti.

ASSISTENZA AL PAZIENTE CON POLMONITE

Professionalità di base
Eseguire un accurato accertamento
infermieristico al momento del ricovero
del paziente affetto da polmonite
Garantire le cure generali: confort, accurata e
corretta nutrizione/idratazione, somministrazione
dei farmaci, gestione dei devices e
dell'ossigenoterapia

\section{Professionalità ottimale}

Pianificare interventi di cura personalizzat

in base al bisogno di assistenza infermieristica, al

livello di autonomia della persona assistita, della sua storia clinica e personale

Dimostrare conoscenze ed abilità nella gestione dei trattamenti farmacologici e non, i devices e i sistemi di somministrazione secondo i protocolli e i bisogni del paziente

Riconoscere le condizioni cliniche che richiedono un intervento diagnostico/terapeutico prioritario (insufficienza respiratoria, febbre, alterazione dello stato di coscienza)

Valutare la frequenza, profondità e qualità del respiro

Osservare e descrivere lo stato della cute

(cianosi), lo stato delle mucose (idratazione),

il letto ungueale (unghie bianche da vasocostrizione)

Conoscere i team di fisioterapia respiratoria attivarli in modo appropriato

Sorvegliare l'insorgenza di complicanze della polmonite

Nella fase post acuta educare e supportare il paziente per incoraggiarlo a prendere decisioni relative alla gestione delle attività della vita quotidiana (alimentazione, attività física, lavoro, attività sessuale, viaggi)

Valutare lo stato mentale, la presenza di irrequietezza, agitazione, confusione e sonnolenza (ipossia cerebrale)

Monitorare la frequenza cardiaca ed il ritmo

Controllare la temperatura corporea, evitando rialzi termici prolungati che aumentano il consumo di ossigeno e la perdita di liquidi, causa di ipotensione e tachicardia, garantendo confort

Far mantenere un decubito semi-fowler, con testa e tronco sollevati a $45^{\circ}$ e cuscino sotto le gambe nei pazienti complicati e fragili

Monitorare la saturimetria

Favorire l'idratazione offrendo bevande calde, per favorire la fluidificazione dell'espettorato

Monitorare la diuresi e il bilancio idrico

Incoraggiare all'assunzione di liquid

$(3000 \mathrm{cc} / \mathrm{die})$ se non controindicato

Monitorare peso e pianificare un programma nutrizionale adeguato per favorire la resistenza all'infezione e la risposta alle terapie

Assistere ed incoraggiare all'igiene del cavo orale dopo terapia con nebulizzatori, eliminan odori e gusti sgradevoli nell'ambiente

Educare a mantenere decubito seduto almeno 30 minuti dopo il pasto

Proporre pasti piccoli e frequenti con cibi graditi per stimolare l'appetito

Valutare le caratteristiche del dolore e registrare attraverso l'uso di scale ed interviene secondo procedure standardizzate

Conoscere e saper gestire le precauzioni standard e specifiche per microrganismo per prevenire le

infezioni correlate all'assistenza (igiene delle mani,

uso dei DPI) secondo i protocolli aziendali

Educare a smettere di fumare $\quad$ Incoraggiare i soggetti ad alto rischio di vaccinarsi

Supportare la persona nelle attività di selfcare

Valutare il contesto e coinvolge i familiari e/o

il cargiver

Educare ed incoraggiare all'igiene delle mani

e del cavo orale per favorire la guarigione $e$

prevenire recidive

Effettuare prelievi per emogasanalisi

Garantire una adeguata igiene ambientale, controllando il calore ed aumentando l'umidità

Promuovere e ricercare strategie per ottenere la massima aderenza terapeutica in collaborazione con l'assistito

Effettuare broncoaspirazioni se indicato

Valutare l'ansia ed incoraggiare alla comunicazione per verbalizzare sentimenti e preoccupazioni tachipnea, respiro superficiale, movimento asimmetrico del torace, la presenza di crepitii, rantoli e sibili

Educare ad esercizi di respirazione profonda ed incoraggiare a mantenere una postura corretta

Identificare i fattori che causano nausea e vomito (espettorato abbondante, dolore, dispnea grave, terapia farmacologica)

Osservare e interpretare la distensione addominale durante l'ossigeno terapia, NIV o areosol terapia massaggi, attivando elementi di distrazione, coinvolgendo il paziente nel controllo del dolore

Stabilire la risposta del paziente all'attività fisica, la percezione dello sforzo (Scala di Borg), la comparsa di dispnea (Scala di mMRC), alterazione dei segni vitali

Provvede ad un progressivo aumento delle attività per promuovere il selfcare attraverso un piano di assistenza personalizzato

Monitorare l'efficacia della terapia farmacologica: se adeguata i segni di miglioramento compaiono dopo $24 / 48$ ore

Educare alla prevenzione di recidive:

evitare folle di persone nei periodi freddi e durante

le epidemie influenzali, a favorire il riposo notturno,

a seguire una dieta sana ed equilibrata,

a fare attività fisica

Scoraggiare all'uso dell'automedicazione,

educando a contattare sempre il medico
Professionalità distintiva

Garantire consulenza ed informazioni sulla cura

ed assistenza della persona affetta da polmonite

Conoscere e comprendere i fattori fisici e psicosociali che influenzano il selfcare nel paziente affetto da polmonite (dispnea, ridotta tolleranza all'attività fisica, dolore toracico) negoziando e pianificando strategie di intervento

Conoscere tutti i trattamenti farmacologici e non e gli eventi avversi degli stessi

Impiantare e gestire gli accessi vascolari (mini-midline, midline, PICC)

Dimostrare conoscenze ed abilità nella gestione del ventilatore per NIV: cambio filtri e circuiti, impostazioni dei parametri di ventilazione, tipi di maschere facciali, presidi per la prevenzione di LdP al volto; così come conoscenze per fornire informazioni competenti al paziente per ottenere la massima compliance durante il trattamento; prevenire e riconoscere le complicanze della NIV Attivare e gestire con abilità le dimissioni diffcili, garantendo continuità delle cure

Educare e supportare il paziente per la gestione, il controllo e la prevenzione delle complicanze

Conoscere e diffondere le linee guida nazionali e internazionali

Partecipare alla ricerca infermieristica per il miglioramento della cura della polmonite

Valutare frequenza, qualità e profondità del respiro: Informare sull'importanza dei follow-up e delle

Partecipare allo sviluppo ed al mantenimento de protocolli e delle procedure sia a livello locale che nazionale per l'assistenza al paziente affetto da polmonite

Assistere favorendo i cambi di posizione, eseguendo 
Tabella 16. Nursing clinical competence: i sanguinamenti gastro-intestinali.

\begin{tabular}{|c|c|c|}
\hline \multicolumn{3}{|c|}{ ASSISTENZA AL PAZIENTE CON SANGUINAMENTI GASTROENTERICI I ${ }^{134-143,360-361}$} \\
\hline Professionalità di base & Professionalità ottimale & Professionalità distintiva \\
\hline Misurare i parametri vitali & Applicare ed impostare monitor multiparametrici & $\begin{array}{l}\text { Programmare, condurre e gestire l'assistenza al } \\
\text { paziente terminale }\end{array}$ \\
\hline $\begin{array}{l}\text { Richiedere esami ematochimici ed effettuare } \\
\text { prelievo venoso }\end{array}$ & $\begin{array}{l}\text { Conoscere ed attivare le procedure di allertamento } \\
\text { dei servizi trasfusionali }\end{array}$ & Posizionare la sonda di Blakemore \\
\hline $\begin{array}{l}\text { Posizionare se necessario un catetere } \\
\text { vescicale }\end{array}$ & Gestire richieste e somministrazione di emazie & $\begin{array}{l}\text { Coordinare/partecipare alla stesura di linee guida } \\
\text { e percorsi assistenziali per pazienti con sanguinamenti } \\
\text { gastro-intestinali }\end{array}$ \\
\hline $\begin{array}{l}\text { Conoscere dove è collocato il materiale per } \\
\text { le emergenze e dove sono i farmaci da frigo }\end{array}$ & $\begin{array}{l}\text { Pianificare la dimissione favorendo la continuità } \\
\text { assistenziale e promuovendo processi educativi } \\
\text { mirati alla prevenzione di recidive }\end{array}$ & $\begin{array}{l}\text { Valutare il rischio post endoscopia con lo Score } \\
\text { di Rockall }\end{array}$ \\
\hline $\begin{array}{l}\text { Assicurare uno o più adeguato accessi venosi } \\
\text { e il loro mantenimento }\end{array}$ & $\begin{array}{l}\text { Preparare e gestire un eventuale trasferimento } \\
\text { in condizioni di urgenza }\end{array}$ & \\
\hline \multicolumn{3}{|l|}{$\begin{array}{l}\text { Conoscere gli aspetti eziologici e fisiopatologici } \\
\text { del sanguinamento gastro-intestinali }\end{array}$} \\
\hline \multicolumn{3}{|l|}{$\begin{array}{l}\text { Monitorare il numero di scariche e loro } \\
\text { caratteristiche }\end{array}$} \\
\hline \multicolumn{3}{|l|}{$\begin{array}{l}\text { Conoscere gli accertamenti diagnostici } \\
\text { per lo studio dei sanguinamenti }\end{array}$} \\
\hline \multicolumn{3}{|l|}{ Effettuare un elettrocardiogramma } \\
\hline \multicolumn{3}{|l|}{ Saper gestire la terapia endovenosa } \\
\hline $\begin{array}{l}\text { Far togliere e conservare occhiali e protesi } \\
\text { dentarie }\end{array}$ & & \\
\hline
\end{tabular}

Tabella 17. Nursing clinical competence: la sepsi.

ASSISTENZA AL PAZIENTE CON SESPSI ${ }^{144-148,362-366}$

\begin{tabular}{l} 
Professionalità di base \\
\hline Effettuare prelievi ematici \\
\hline Preparare e somministrare terapia antimicrobica
\end{tabular}

\section{Professionalità ottimale}

Gestire i presidi per ossigenoterapia ad alti flussi

Organizzare l'informazione del paziente e

dei famigliari riguardo il follow-up

Stratificare il livello di rischio attraverso la scala qSOFA

Identificare possibili fonti dell'infezione

Identificazione dei fattori che incrementano

il rischio di sviluppare sepsi

Riconoscere precocemente segni e sintomi

disfunzione d'organo

\section{Raccogliere campioni per esecuzione di}

esami culturali di routine

Posizionare se indicato e gestire il catetere

vescicale

Gestire correttamente un catetere venoso centrale

Somministrare correttamente emoderivati

Monitorare e registrare le risposte alla terapia

Rispettare le misure per il controllo delle infezioni

Programmare ed effettuare cure igieniche

del cavo orale

Ispezionare regolarmente la cute per

riconoscimento segni di ipoperfusione

Impostare e utilizzare pompe infusionali

volumetriche e pompe a siringa

Monitorare ecchimosi e sanguinamento

Tabella 18. Nursing clinical competence: il monitoraggio cardiaco.

\begin{tabular}{lll}
\hline Professionalità di base & \multicolumn{1}{c}{ IL MONITORAGGIO CARDIACO ${ }^{75-80,149}$} & Professionalità distintiva \\
\hline $\begin{array}{l}\text { Posizionare ed impostare i sistemi di } \\
\text { monitoraggio }\end{array}$ & $\begin{array}{l}\text { Cogliere precocemente modificazioni del } \\
\text { ritmo cardiaco }\end{array}$ & Riconoscere e descrivere un tracciato patologico \\
\hline $\begin{array}{l}\text { Valutare la qualità del tracciato riprodotto } \\
\text { Utilizzare il defibrillatore semi-automatico }\end{array}$ & $\begin{array}{l}\text { Conoscere i farmaci pro aritmici di uso più } \\
\text { comune }\end{array}$ & $\begin{array}{l}\text { Conoscere la procedura e l'assistenza per la } \\
\text { cardioversione elettrica }\end{array}$ \\
\hline Ruando necessario & $\begin{array}{l}\text { Conoscere i protocolli dei farmaci per la } \\
\text { cardioversione farmacologica }\end{array}$ & Conoscere i protocolli ALS \\
\hline Riconoscere le aritmie maligne $(\mathrm{FV}, \mathrm{TV}, \ldots)$ & Riconoscere le aritmie maggiori & \\
\hline
\end{tabular}


Tabella 19. Nursing clinical competence: la ventilazione non-invasiva.

\begin{tabular}{|c|c|c|}
\hline \multicolumn{3}{|c|}{ LA VENTILAZIONE NON INVASIVA ${ }^{151-155,367}$} \\
\hline Professionalità di base & Professionalità ottimale & Professionalità distintiva \\
\hline Conoscere le indicazioni per la NIV negli adulti & $\begin{array}{l}\text { Conoscere le controindicazioni relative per } \\
\text { la NIV negli adulti }\end{array}$ & $\begin{array}{l}\text { Conoscere il significato delle curve e dei grafici } \\
\text { del ventilatore }\end{array}$ \\
\hline $\begin{array}{l}\text { Conoscere e rispettare le controindicazioni } \\
\text { assolute per la NIV negli adulti }\end{array}$ & $\begin{array}{l}\text { Impostare ed interpretare gli allarmi in base } \\
\text { alle impostazioni del ventilatore ed allo stato } \\
\text { clinico del paziente }\end{array}$ & Redigere protocolli di gestione del paziente in NIV \\
\hline Conoscere le funzioni principali del ventilatore & $\begin{array}{l}\text { Conoscere le varie modalità di ventilazione } \\
\text { (CPAP e BiPAP) }\end{array}$ & $\begin{array}{l}\text { Variare le modalità del ventilatore durante lo } \\
\text { svezzamento }\end{array}$ \\
\hline $\begin{array}{l}\text { Riconoscere e rispondere prontamente } \\
\text { agli allarmi }\end{array}$ & $\begin{array}{l}\text { Riconoscere i principali meccanismi } \\
\text { fisiopatologici sottesi al quadro clinico }\end{array}$ & $\begin{array}{l}\text { Monitorare la qualità assistenziale dei pazienti } \\
\text { in trattamento con NIV }\end{array}$ \\
\hline $\begin{array}{l}\text { Conoscere le modalità di montaggio e prove } \\
\text { di tenuta del circuito respiratorio }\end{array}$ & Gestire la rotazione delle interfacce & Gestire i presidi sovra-glottici \\
\hline $\begin{array}{l}\text { Conoscere le interfacce disponibili con relativi } \\
\text { vantaggi e svantaggi }\end{array}$ & Formulare le ipotesi diagnostiche infermieristiche & $\begin{array}{l}\text { Contribuire alla formazione efficace degli studenti } \\
\text { e colleghi neo-inseriti }\end{array}$ \\
\hline $\begin{array}{l}\text { Applicare il divieto di contenzione in pazienti } \\
\text { in ventilazione non invasiva }\end{array}$ & $\begin{array}{l}\text { Riconoscere il deterioramento clinico e le } \\
\text { indicazioni al cambio di modalità }\end{array}$ & Esercitare la funzione di tutor dei colleghi neo-inseriti \\
\hline $\begin{array}{l}\text { Conoscere le principali complicanze } \\
\text { (secchezza mucose, congestione nasale, } \\
\text { irritazione, distensione gastrica e lesioni } \\
\text { cutanee) ed azioni preventive }\end{array}$ & $\begin{array}{l}\text { Saper discriminare il paziente suscettibile } \\
\text { di terapia non invasiva da quello che richiede } \\
\text { intervento intensivo }\end{array}$ & Condurre un audit clinico \\
\hline $\begin{array}{l}\text { Applicare la scala di Kelly e Matthay per la } \\
\text { valutazione dello stato neurologico }\end{array}$ & $\begin{array}{l}\text { Allertare il medico sui segni/sintomi ed } \\
\text { accertamenti predittivi di evoluzione negativa }\end{array}$ & Partecipare ad iniziative di miglioramento della qualità \\
\hline Applicare un monitoraggio multiparametrico & Calcolare il rapporto $\mathrm{P} / \mathrm{F}$ & $\begin{array}{l}\text { Realizzare report di aggiornamento della letteratura } \\
\text { sulla NIV }\end{array}$ \\
\hline $\begin{array}{l}\text { Riconoscere segni e sintomi indicativi di } \\
\text { instabilità del quadro clinico }\end{array}$ & $\begin{array}{l}\text { Valutare la capacità di coping del paziente } \\
\text { e dei familiari per deficit di conoscenza ed } \\
\text { ansia riguardo al trattamento in NIV }\end{array}$ & $\begin{array}{l}\text { Partecipare alla stesura di linee guida e PDTA } \\
\text { per i pazienti in NIV }\end{array}$ \\
\hline $\begin{array}{l}\text { Fornire le informazioni necessarie al paziente } \\
\text { ed ai familiari }\end{array}$ & $\begin{array}{l}\text { Definire il grado di umidità e calore nella miscela } \\
\text { di aria a seconda delle necessità del paziente }\end{array}$ & \\
\hline \multicolumn{3}{|l|}{ Rassicurare il paziente } \\
\hline \multicolumn{3}{|l|}{$\begin{array}{l}\text { Istruire il paziente per la rimozione rapida } \\
\text { in caso di problemi }\end{array}$} \\
\hline $\begin{array}{l}\text { Coinvolgere il paziente/familiari nella } \\
\text { gestione del processo di cura }\end{array}$ & & \\
\hline
\end{tabular}


Tabella 20. Nursing clinical competence: l'ecografia operativa bedside.

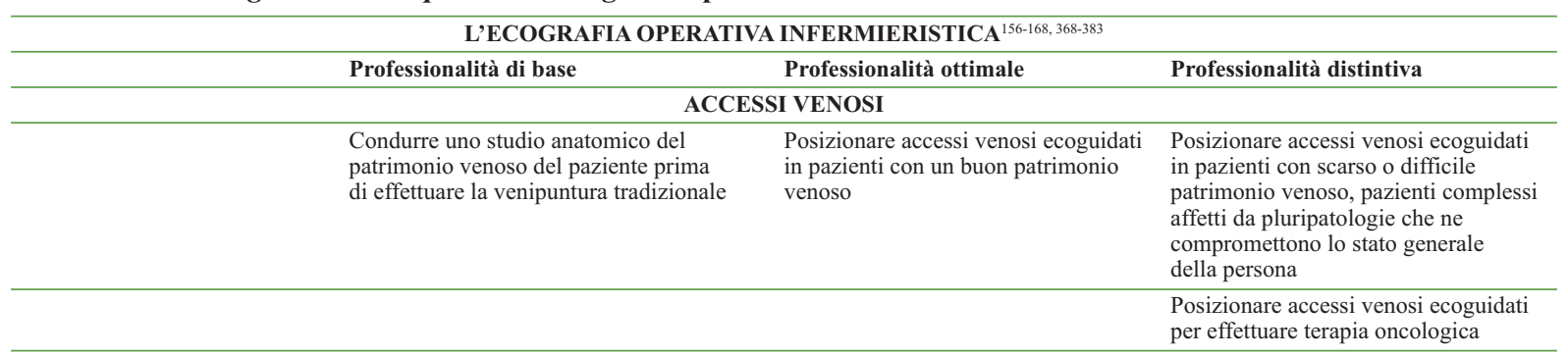

VALUTAZIONE PAZIENTE SCOMPENSATO

Visualizzazione pleura $\quad$ Visualizzare la pleura del parenchima

polmonare sapendo riconoscere un fisiologico sliding dei foglietti parietali, distinguendo le immagine ecografiche del polmone wet e sapendo evidenziare le alterazioni caratteristiche, le linee B che sottendono a quadri degenerativi (quali le polmoniti)

Visualizzazione pericardio

Visualizzazione parenchima

renale

Valutazione vena cava
Effettuare una scansione del pericardio in pazienti con difficoltà respiratoria per evidenziare eventual versamenti

Effettuare una valutazione parenchima renale in pazienti traumatizzati per escludere versamenti

Effettuare valutazioni delle dimensioni della vena cava in pazienti scompensat per raccogliere dati precisi sul suo stato d'idratazione

\section{ECOGRAFIA VESCICALE}

Effettuare una valutazione vescicale ecografica in caso di ritenzione urinaria sapendo evidenziare quadri di alterazione della funzionalità fisiologica visualizzandone il globo vescicale

Valutare il corretto posizionamento del catetere vescicale tramite la visualizzazione del palloncino in vescica
Valutare alterazioni della

funzionalità vescicale favorenti

l'insorgere delle infezioni

visualizzando eventuale ristagno urinario post minzione
Valutare il paziente scompensato tramite la scansione di immagini ecografiche della pleura bilaterale, del pericardio, del parenchima renale e della vena cava al fine di rivalutare lo stato di idratazione del paziente ed eventuali versamenti

addominali polmonari o cardiaci sia in caso di trauma che in quello d'acuzia della patologia cardiaca o nel follow up dei pazienti affetti da scompenso cardiaco

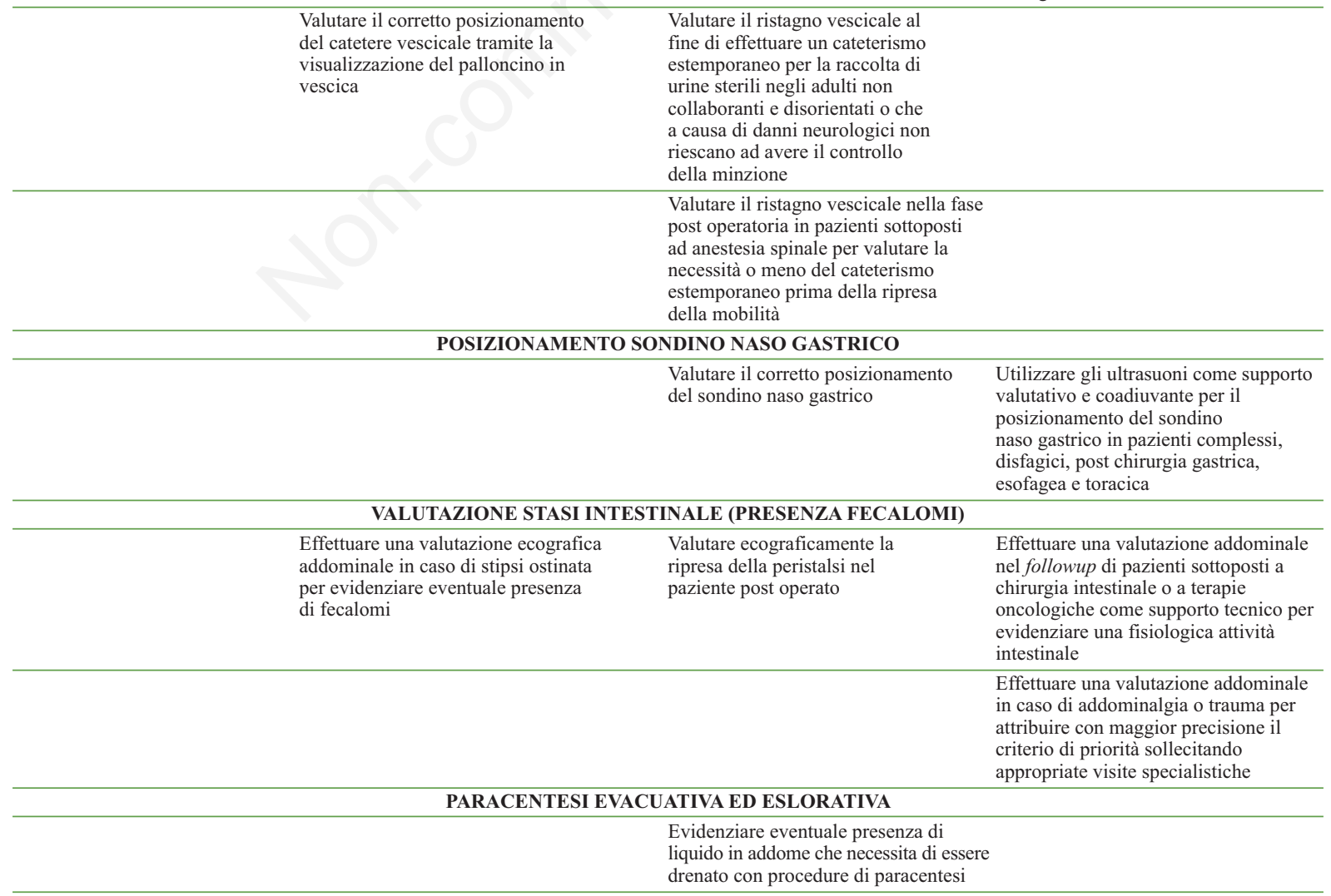

Condurre un supporto valutativo in pazienti complessi o sottoposti a chirurgia urologica per evidenziare difficoltà determinate da ipertrofie prostatiche o secondari ad interventi urologici

Valutare il ristagno vescicale a fine di effettuare un cateterismo mporaneo per la raccolta di e sterili negli adulti non collaboranti e disorientati o ch causa di danni neurologici non della minzione

alutare il ristagno vescicale nella fase post operatoria in pazienti sottoposti anestesia spinale per valutare necessità o meno del cateterismo estemporaneo prima della ripresa DINO NASO GASTRICO

Valutare il corretto posizionamento valutativo e coadiuvante per il isfagici, post chirurgia gastrica, el followup di pazienti sottoposti chirurgia intestinale o a terapie oncologiche come supporto tecnico pe intestinal

Effettuare una valutazione addominale caso di addominalgia o trauma per

criterio di priorità sollecitando appropriate visite specialistiche 
Tabella 21. Nursing clinical competence: somministrazione di terapia e chemioterapia.

LA SOMMINISTRAZIONE DI TERAPIA E CHEMIOTERAPIA ${ }^{169-205,384-387}$

\section{Professionalità di base}

Conoscere e mettere in pratica le regole per una somministrazione sicura attraverso le vie e modalità di somministrazione utilizzate comunemente nel proprio setting un'assunzione sicura dei chemioterapici per os (in particolare: manipolazione, interazione con cibi/farmaci/stile di vita, assunzione)
Conoscere e mettere in pratica le regole per

\section{Professionalità ottimale}

Conoscere la classificazione e il meccanismo di azione dei farmaci antineoplastici

Rivalutare periodicamente e in relazione a persona (usando sistemi quali il conteggio delle compresse, ecc) e impostare interventi educativi e di supporto in caso di necessità (promemoria sms, utilizzo di calendari) cambiamenti clinici, il grado di aderenza della

Professionalità distintiva

Segnalare gli eventi avversi agli organismi di farmacovigilanza

Valutare la prontezza e la capacità di apprendere della persona (attraverso l'indagine su: età e livello di sviluppo, conoscenze di partenza, attese rispetto a malattia e trattamento, risposta alle nuove conoscenze, capacità e desiderio di autocura) e conseguente impostazione della velocità/passo di insegnamento riguardo al proprio schema di trattamento, prevedendo rivalutazioni e rinforzi periodici

Conoscere l'importanza dell'aderenza terapeutica Saper utilizzare tempestivamente e correttamente del paziente al piano di cura e utilizzare correttamente il kit per lo spandimento di antiblastici la scala di valutazione Morisky

Conoscere e mettere in pratica le regole per una gestione sicura dei chemioterapici per via endovenosa (in bolo e in infusione)

Per la somministrazione di antiblastici: conoscere i principi generali di controllo (a due operatori) della correttezza di: prescrizione (regola delle $7 \mathrm{G}$ in relazione al ciclo di chemioterapia, necessità di terapie ancillari o idratazione), farmaco preparato dall'Unità di Manipolazione dei Chemioterapici Antiblastici attraverso il controllo dell'etichettatura, e dell'identificazione del paziente (attraverso l'uso di due identificatori, es. nome e data di nascita) Conoscere e adottare principi e requisiti per una manipolazione sicura dei chemioterapici, della conservazione e stoccaggio, dei relativi rifiuti e di eventuali liquidi biologici contaminati (dispositivi di protezione individuale appropriati rispetto alla via di somministrazione e in base agli standard nazionali, smaltimento dei rifiuti)

Conoscere ubicazione, contenuto e principi di utilizzo di kit per lo spandimento

Conoscere, monitorare e/o verificare la presenza di parametri generali, prima della somministrazione della terapia antiblastica $\left(\mathrm{PA}, \mathrm{FC}, \mathrm{SpO}_{2} \mathrm{TC}\right.$, peso corporeo, altezza e superficie corporea o BMI) Conoscere e adottare principi per la prevenzione e gestione di sintomi ed effetti collaterali generali a breve/medio/lungo termine (nausea e vomito, mucosite e diarrea): attuazione della profilassi antiemetica prescritta e uso del protocollo sull'igiene orale
Conoscere e adottare principi per la prevenzione, identificazione precoce, monitoraggio e gestione di sintomi ed effetti collaterali generali farmaco-specific

Conoscere e adottare principi per prevenzione, identificazione, monitoraggio e gestione di tossicita ed eventi avversi associati a chemioterapia

Valutare il patrimonio venoso del paziente in relazione al piano terapeutico, ai farmaci da somministrare, alla compliance e capacità di self-management di paziente e caregiver
Reperire accesso venoso centrale ad inserzione periferica per la somministrazione di terapie vescicanti in continuo o per lunghi periodi di tempo

Coordinare il team multidisciplinare in modo da assicurare che le cure siano fornite nel setting più adeguato e sicuro, facendosi portavoce del paziente rispetto all'equipe e viceversa

Utilizzare frequentemente e partecipare attivamente alla ricerca scientifica (identificazione di problemi quesiti di ricerca e di gap nell'assistenza, valutazione critica degli articoli, supporto ai trial clinici, lavoro in gruppi interprofessionali per cercare risposte evidence-based, partecipazione ad associazion professionali pubblicando il proprio lavoro)
Valutare e utilizzare il materiale di somministrazione

della terapia endovenosa più idoneo in relazione a:

protocollo di trattamento, preferenze della persona e risorse disponibili

Educare paziente e caregiver su: comportamenti da adottare per limitare il rischio di dislocazione del device e sull'importanza di una comunicazione tempestiva dell'eventuale comparsa di sintomi

Registrare in modo preciso e coerente: outcomes (clinici, educativi, ecc), conoscenze, giudizi significativi e il pensiero critico degli operatori sul singolo paziente, per garantire un'assistenza personalizzata durante tutto il processo terapeutico. La documentazione deve includere: storia clinica e fisiologica; valutazione fisica; valutazione e intervent sugli eventuali effetti collaterali e sintomi; valutazione psicosociale/spirituale/culturale, sessualità; informazioni necessarie al team per garantire un'assistenza personalizzata; schema terapeutico, informazioni fornite al paziente e alle eventuali esigenze formative in merito; contatti e rapporti per la continuità assistenziale

Conoscere i principi per la scelta, la valutazione di appropriatezza e del corretto funzionamento del device vascolare scelto $\mathrm{o}$ già presente Conoscere le tecniche ed i sistemi per favorire la continuita delle cure nel contesto territoriale in cui opera, compresa l'organizzazione de rifornimento del materiale occorrente al paziente per il self-care (farmaci, aghi, pompe, ecc) e aiutando la persona ad intraprendere eventuali percorsi di supporto (es. psicologico, sociale)

Saper reperire accesso venoso periferico idoneo all'uso e saper gestire correttamente eventuale accesso venoso centrale (cvc, picc o port)

Conoscere e saper utilizzare in modo idoneo il materiale necessario alla somministrazione endovenosa (set di infusione e pompe infusionali), prediligendo raccordi luer-lock e sistemi a circuito chiuso prevenire la contaminazione

Conoscere e adottare principi e requisiti generali per la prevenzione dello stravaso

Saper registrare in modo preciso e coerente le informazioni raccolte (raccolte dati, pianificazioni, interventi e valutazioni), utilizzando la documentazione prevista nella struttura

Conoscere, consultare e adottare i protocolli di riferimento nella propria struttura

Essere consapevole del limite delle proprie

competenze evitando di gestire situazioni nella quali

si rilevano le proprie carenze o incapacità, avvalendosi

del confronto con personale più esperto 
Tabella 22. Nursing clinical competence: le lesioni da pressione.

\begin{tabular}{|c|c|c|}
\hline \multicolumn{3}{|c|}{ LE LESIONI DA PRESSIONE ${ }^{206-225}$} \\
\hline Professionalità di base & Professionalità ottimale & Professionalità distintiva \\
\hline $\begin{array}{l}\text { Riconoscere e classificare i } 4 \text { stadi di lesione } \\
\text { da pressione }\end{array}$ & $\begin{array}{l}\text { Conoscere le complicanze relative alle lesioni } \\
\text { (intrinseche ed estrinseche) e saperle prevenire }\end{array}$ & $\begin{array}{l}\text { Stabilire il livello di cura per il paziente: cura attiva o } \\
\text { palliativa in base alle condizioni generali e aspettative } \\
\text { del paziente }\end{array}$ \\
\hline $\begin{array}{l}\text { Conoscere ed utilizzare le medicazioni di base } \\
\text { (poliuretano, film, creme protettive) }\end{array}$ & $\begin{array}{l}\text { Eseguire una valutazione del paziente a rischio } \\
\text { con relativa programmazione dell'assistenza }\end{array}$ & $\begin{array}{l}\text { Trattare tutti i tipi di lesione con le adeguate } \\
\text { medicazioni e i siti peri-lesionali in collaborazione } \\
\text { con l'equipe medica e chirurgica }\end{array}$ \\
\hline $\begin{array}{l}\text { Applicare la corretta medicazione in base } \\
\text { alla prescrizione medica }\end{array}$ & $\begin{array}{l}\text { Identificare le lesioni necrotiche e } \\
\text { eventualmente infette }\end{array}$ & $\begin{array}{l}\text { Identificare e prevenire le complicanze reali } \\
\text { o potenziali }\end{array}$ \\
\hline $\begin{array}{l}\text { Conoscere ed applicare le scale di } \\
\text { prevenzione delle lesioni (Braden) e le scale } \\
\text { di valutazione del dolore (VAS, VNS, NRS) }\end{array}$ & $\begin{array}{l}\text { Programmare e inviare la consulenza chirurgica } \\
\text { e/o dell'infermiere esperto per eventuale } \\
\text { toilettatura }\end{array}$ & Scegliere la medicazione avanzata più corretta \\
\hline \multirow[t]{3}{*}{$\begin{array}{l}\text { Conoscere le principali tecniche di prevenzione } \\
\text { (mobilizzazione a letto, utilizzo presidi } \\
\text { antidecubito) }\end{array}$} & $\begin{array}{l}\text { Programmare in equipe il piano di dimissione } \\
\text { con relativo schema di movimentazione e } \\
\text { scelta di presidi e medicazioni adeguati per } \\
\text { il domicilio }\end{array}$ & $\begin{array}{l}\text { Pianificare dopo la dimissione controlli periodici } \\
\text { per rivalutare le medicazioni }\end{array}$ \\
\hline & & $\begin{array}{l}\text { Organizzare e gestire ambulatori infermieristici } \\
\text { per la cura delle lesioni da pressione }\end{array}$ \\
\hline & & $\begin{array}{l}\text { Organizzare e pianificare corsi di aggiornamento } \\
\text { per la propria equipe }\end{array}$ \\
\hline
\end{tabular}

Tabella 23. Nursing clinical competence: il dolore.

\begin{tabular}{|c|c|c|}
\hline \multicolumn{3}{|c|}{ ASSISTENZA AL PAZIENTE CON DOLORE ${ }^{226-240}$} \\
\hline Professionalità di base & Professionalità ottimale & Professionalità distintiva \\
\hline $\begin{array}{l}\text { Osservare e rilevare variazioni del paziente } \\
\text { riguardo a comportamento, andatura, attività, } \\
\text { modello sonno-veglia, appetito, agitazione }\end{array}$ & $\begin{array}{l}\text { Conoscere e gestire le interazioni della terapia } \\
\text { antalgica con i farmaci più comunemente } \\
\text { utilizzati dagli anziani }\end{array}$ & $\begin{array}{l}\text { Educare il paziente e caregivers ad utilizzare anche } \\
\text { strategie non farmacologiche per il controllo del dolore, } \\
\text { come tecniche di rilassamento, massaggi, } \\
\text { terapie complementari }\end{array}$ \\
\hline $\begin{array}{l}\text { Conoscere e utilizzare le scale per la rilevazione } \\
\text { e valutazione del dolore }\end{array}$ & $\begin{array}{l}\text { Valutare e registrare come il dolore interferisce } \\
\text { nelle attività quotidiane del paziente, sulla sua } \\
\text { qualità di vita, sul sonno, sull'attività sessuale, } \\
\text { sul ruolo sociale e sull'impiego }\end{array}$ & $\begin{array}{l}\text { Valutare ed esplorare credenze e cultura della persona, } \\
\text { età, livello d'istruzione, etnia, spiritualità nella } \\
\text { pianificazione dell'assistenza }\end{array}$ \\
\hline $\begin{array}{l}\text { Rilevare e documentare intensità e caratteristiche } \\
\text { del dolore, fattori che lo aumentano e diminuiscono }\end{array}$ & $\begin{array}{l}\text { Stabilire un piano di cura che incorpori gli } \\
\text { obiettivi della persona e del team } \\
\text { multiprofessionale }\end{array}$ & \\
\hline $\begin{array}{l}\text { Registrare le terapie e i tratttamenti assunti, } \\
\text { l'aderenza, l'efficacia }\end{array}$ & $\begin{array}{l}\text { Anticipare e trattare il dolore prima, durante } \\
\text { e dopo trattamenti diagnostico-terapeutici } \\
\text { invasivi e/o dolorosi }\end{array}$ & \\
\hline $\begin{array}{l}\text { Valutare e registrare se storia di abusi e dipendenze } \\
\text { da sostanze o farmaci }\end{array}$ & $\begin{array}{l}\text { Coinvolgere la persona, la sua famiglia, } \\
\text { i caregivers rispetto il piano di cura e le } \\
\text { strategie da attuare }\end{array}$ & \\
\hline $\begin{array}{l}\text { Conoscere e gestire i principali effetti avversi } \\
\text { correlati alla terapia del dolore }\end{array}$ & $\begin{array}{l}\text { Educare la persona e la famiglia a comprendere } \\
\text { lo scopo di ciascun farmaco, il momento appropriato } \\
\text { in cui usarlo, gli effetti collaterali, le strategie } \\
\text { che possono essere adottate per prevenirli } \\
\text { e ricordare di tenere sempre i farmaci lontano } \\
\text { dalla portata dei bambini }\end{array}$ & \\
\hline \multicolumn{3}{|l|}{$\begin{array}{l}\text { Comunicare e documentare le risposte della } \\
\text { persona al piano di gestione del dolore }\end{array}$} \\
\hline $\begin{array}{l}\text { Valutare la presenza di dolore regolarmente } \\
\text { e frequentemente, entro un'ora dalla } \\
\text { somministrazione del trattamento, in seguito } \\
\text { almeno ogni } 4 \text { ore }\end{array}$ & & \\
\hline
\end{tabular}


Tabella 24. Nursing clinical competence: gli accessi vascolari.

\begin{tabular}{|c|c|c|}
\hline \multicolumn{3}{|c|}{ GLI ACCESSI VASCOLARI ${ }^{241-251,388-401}$} \\
\hline Professionalità di base & Professionalità ottimale & Professionalità distintiva \\
\hline $\begin{array}{l}\text { Utilizzare scale di valutazione del patrimonio } \\
\text { venoso nel paziente ospedalizzato }\end{array}$ & $\begin{array}{l}\text { Conoscere i devices disponibili sul mercato } \\
\text { e scegliere in base alle caratteristiche necessarie }\end{array}$ & Applicare i principi base dell'ecografia venosa \\
\hline $\begin{array}{l}\text { Pianificare la necessità di un accesso venoso } \\
\text { (extra ospedaliero) alla dimissione, già al } \\
\text { momento dell'ammissione in ospedale }\end{array}$ & $\begin{array}{l}\text { Riconoscere le controindicazioni al } \\
\text { posizionamento di un catetere periferico }\end{array}$ & Saper posizionare un catetere midline o un PICC \\
\hline $\begin{array}{l}\text { Identificare precocemente (all'ingresso in ospedale) } \\
\text { i pazienti con scarso patrimonio venoso }\end{array}$ & Gestire il dolore durante e dopo l'inserzione & $\begin{array}{l}\text { Effettuare il controllo ecografico della vena } \\
\text { giugulare durante l'inserzione di un PICC }\end{array}$ \\
\hline $\begin{array}{l}\text { Informare il paziente sulla procedura ed } \\
\text { ottenerne il consenso }\end{array}$ & $\begin{array}{l}\text { Scegliere il metodo di fissaggio appropriato } \\
\text { (suturless device) }\end{array}$ & $\begin{array}{l}\text { Utilizzare il metodo dell'ECG intracavitario per } \\
\text { verificare la posizione della punta del PICC }\end{array}$ \\
\hline $\begin{array}{l}\text { Posizionare i dispositivi venosi periferici con } \\
\text { tecnica asettica }\end{array}$ & $\begin{array}{l}\text { Riconoscere tempestivamente una trombosi } \\
\text { catetere-correlata }\end{array}$ & $\begin{array}{l}\text { Identificare la punta del PICC in una radiografia } \\
\text { del torace }\end{array}$ \\
\hline $\begin{array}{l}\text { Istruire il paziente circa il monitoraggio e il } \\
\text { riconoscimento delle complicanze }\end{array}$ & $\begin{array}{l}\text { Istruire il personale neo-assunto il riconoscimento } \\
\text { dei segni e sintomi di ogni complicanza }\end{array}$ & $\begin{array}{l}\text { Saper riconoscere occlusioni venose con l'ausilio } \\
\text { dell'ecografia }\end{array}$ \\
\hline Prevenire le occlusioni del catetere venoso & & $\begin{array}{l}\text { Effettuare una disostruzione del catetere venoso, } \\
\text { in base alla natura dell'occlusione, ove le condizioni } \\
\text { lo consentano }\end{array}$ \\
\hline $\begin{array}{l}\text { Monitorare il sito di inserzione del catetere } \\
\text { almeno ogni sei ore }\end{array}$ & & $\begin{array}{l}\text { Coordinare/partecipare attivamente alla stesura } \\
\text { di protocolli e percorsi assistenziali per rendere } \\
\text { efficiente ed efficace l'assistenza ai pazienti con } \\
\text { cateteri venosi e la loro gestione }\end{array}$ \\
\hline \multirow{2}{*}{\multicolumn{2}{|c|}{$\begin{array}{l}\text { Identificare le terapie e le soluzioni che non } \\
\text { possono essere somministrate in vena periferica }\end{array}$}} & $\begin{array}{l}\text { Partecipare ad iniziative di miglioramento della } \\
\text { qualità per una efficace prevenzione, riconoscimento } \\
\text { precoce e riduzione delle possibili complicanze }\end{array}$ \\
\hline & & $\begin{array}{l}\text { Realizzare periodici report di aggiornamento della } \\
\text { letteratura scientifica sull'argomento }\end{array}$ \\
\hline
\end{tabular}

Tabella 25. Nursing clinical competence: il paziente fragile.

\begin{tabular}{|c|c|c|}
\hline \multicolumn{3}{|c|}{ ASSISTENZA AL PAZIENTE FRAGILE ${ }^{252-263}$} \\
\hline Professionalità di base & Professionalità ottimale & Professionalità distintiva \\
\hline $\begin{array}{l}\text { Identificare segni e sintomi di fragilità nel } \\
\text { paziente con plurimorbilità (astenia, debolezza } \\
\text { muscolare, ridotta velocità nella deambulazione, } \\
\text { riduzione dell'attività fisica, perdita di peso) }\end{array}$ & $\begin{array}{l}\text { Riconoscere precocemente i segni di costipazione, } \\
\text { dolore e ipossia nel paziente con delirium }\end{array}$ & $\begin{array}{l}\text { Coordinare piani di assistenza al paziente fragile } \\
\text { in equipe multidisciplinari (fisioterapista, } \\
\text { logopedista, ...) }\end{array}$ \\
\hline Valutare la presenza di sarcopenia & $\begin{array}{l}\text { Impostare e valutare l'aderenza di piani di } \\
\text { mobilizzazione precoce nel paziente allettato }\end{array}$ & Implementare le cure con CAM nel paziente demente \\
\hline $\begin{array}{l}\text { Applicare scale di valutazione del rischio } \\
\text { di malnutrizione }\end{array}$ & $\begin{array}{l}\text { Concordare obiettivi di riattivazione raggiungibili } \\
\text { con il paziente, i familiari/caregivers }\end{array}$ & $\begin{array}{l}\text { Effettuare con il medico periodiche ricognizioni } \\
\text { terapeutiche in modo da ridurre le terapie al domicilio, } \\
\text { diminuendo i rischi correlati alla polifarmacoterapia }\end{array}$ \\
\hline $\begin{array}{l}\text { Pianificare l'assistenza valutando le principali } \\
\text { alterazioni umorali evitando l'insorgenza di eventi } \\
\text { infausti (cadute) }\end{array}$ & $\begin{array}{l}\text { Favorire al paziente fragile un sonno continuo } \\
\text { durante la notte, riducendo pisolini diurni }\end{array}$ & \\
\hline \multicolumn{3}{|l|}{$\begin{array}{l}\text { Gestire e ridurre episodi di stitichezza e dolore } \\
\text { nel paziente demente }\end{array}$} \\
\hline $\begin{array}{l}\text { Pianificare l'assistenza considerando il rischio } \\
\text { di sindrome da allettamento e ridurre i principali } \\
\text { rischi (ipotensione ortostatica, lesioni da pressione, } \\
\text { delirium, incontinenza, cadute) }\end{array}$ & & \\
\hline
\end{tabular}


Tabella 26. Nursing clinical competence: la dimissione difficile.

\begin{tabular}{|c|c|c|c|}
\hline \multicolumn{4}{|c|}{ LA DIMISSIONE DIFFICILE ${ }^{264-270,402-408}$} \\
\hline Ambiti di attività & Professionalità di base & Professionalità ottimale & Professionalità distintiva \\
\hline \multirow[t]{4}{*}{ Screening } & Identificare i caregivers e i loro ruoli & $\begin{array}{l}\text { Identificare fattori di rischio per } \\
\text { una dimissione difficile del paziente } \\
\text { in medicina interna }\end{array}$ & $\begin{array}{l}\text { Monitorare i fattori di rischio e i } \\
\text { progressi del paziente durante tutto } \\
\text { il percorso e nel suo variare dei setting }\end{array}$ \\
\hline & $\begin{array}{l}\text { Identificare i principali indicatori } \\
\text { sociali che modificano il rischio di } \\
\text { dimissione difficile }\end{array}$ & $\begin{array}{l}\text { Riconoscere fattori di rischio specifici } \\
\text { al variare del setting }\end{array}$ & \\
\hline & $\begin{array}{l}\text { Condurre in maniera guidata un } \\
\text { accertamento iniziale }\end{array}$ & & \\
\hline & $\begin{array}{l}\text { Utilizzare la Scala Brass all'inizio } \\
\text { del ricovero e alla fine }\end{array}$ & & \\
\hline \multirow[t]{5}{*}{ Attori dell'organizzazione } & $\begin{array}{l}\text { Identificare tutti i professionisti coinvolti } \\
\text { nel percorso di dimissione: medico, } \\
\text { coordinatore infermieristico, nuclei di } \\
\text { dimissioni difficili, Case/Care Manager, } \\
\text { primary nurse, assistente sociale, terapisti, } \\
\text { ministro di culto }\end{array}$ & $\begin{array}{l}\text { Identifica e riconosce tutti i possibili } \\
\text { servizi necessari per il percorso } \\
\text { di dimissione: servizi territoriali, } \\
\text { attivazione ausili e presidi, piani } \\
\text { terapeutici, farmacia, dimissione } \\
\text { protetta, follow-up ambulatoriale }\end{array}$ & $\begin{array}{l}\text { Coordinare equipe per le } \\
\text { dimissioni complesse }\end{array}$ \\
\hline & $\begin{array}{l}\text { Attivare l'infermiere specializzato } \\
\text { per il percorso di dimissione quando } \\
\text { necessario }\end{array}$ & & $\begin{array}{l}\text { Progettare percorsi di dimissione } \\
\text { difficile attivando tutti i professionisti } \\
\text { ed i servizi necessari }\end{array}$ \\
\hline & & & $\begin{array}{l}\text { Condurre progetti di ricerca qualitativa } \\
\text { circa i processi di dimissione }\end{array}$ \\
\hline & & & $\begin{array}{l}\text { Sviluppare percorsi di formazione } \\
\text { per il personale }\end{array}$ \\
\hline & & & $\begin{array}{l}\text { Effettuare periodici debriefing delle } \\
\text { dimissioni difficili come strategia di } \\
\text { miglioramento della qualità. Monitorare } \\
\text { i tassi di reospedalizzazioni }\end{array}$ \\
\hline Relazioni & & $\begin{array}{l}\text { Adattare le modalità d'approccio } \\
\text { in base alla cultura del paziente e } \\
\text { del suo contesto familiare }\end{array}$ & \\
\hline \multirow[t]{5}{*}{ Coinvolgere pazienti e cargivers } & $\begin{array}{l}\text { Utilizzare scale quali Health Care } \\
\text { Empowerment Inverty, Patirnt } \\
\text { Activation Measurement, Goal } \\
\text { Attainment Scalling per misurare il } \\
\text { grado di coinvolgimento }\end{array}$ & $\begin{array}{l}\text { Coinvolgere i caregivers nel processo } \\
\text { facendolo partecipare a in modo attivo } \\
\text { (riunioni) }\end{array}$ & \\
\hline & $\begin{array}{l}\text { Concordare gli obiettivi di cura, } \\
\text { considerando i valori del paziente } \\
\text { e dei caregivers }\end{array}$ & & \\
\hline & Raccoglie volontà scritte o non documentate & & \\
\hline & $\begin{array}{l}\text { Valutare il supporto sociale, la presenza } \\
\text { o meno di caregivers, l'utilizzo o meno di } \\
\text { risorse comunitarie }\end{array}$ & & \\
\hline & $\begin{array}{l}\text { Valutare la necessità dei caregivers } \\
\text { attraverso la Next Step in Care } \\
\text { Assessment, conversazioni guidate } \\
\text { ed esplorare: disponibilità, preparazione } \\
\text { e preoccupazioni }\end{array}$ & & \\
\hline \multirow[t]{2}{*}{ Gestione rischi e sintomi } & $\begin{array}{l}\text { Identificare e monitorare sintomi e fattori } \\
\text { di rischio clinici legati alle patologie } \\
\text { di base }\end{array}$ & $\begin{array}{l}\text { Identificare le possibili barriere } \\
\text { che possano ostacolare la dimissione } \\
\text { e correggerle }\end{array}$ & $\begin{array}{l}\text { Sviluppare un piano di dimissione prima } \\
\text { dell'ammissione in ospedale di accessi } \\
\text { programmati }\end{array}$ \\
\hline & $\begin{array}{l}\text { Utilizzare scale validate per la } \\
\text { stratificazione di rischi specifici, } \\
\text { ad esempio: stato cognitivo } \\
\text { (Six Item Screen); delirio (Confusion } \\
\text { Assessment Method Diagnostic Algorithm } \\
\text { o Family CAM); stato funzionale (Basic } \\
\text { Activities of Daily Living o Instrumental } \\
\text { Activities of Daily Living); abuso di sostanze } \\
\text { (Alcohol Use Disorder Identification Test); } \\
\text { nutrizione (Mini nutritional Assestment, } \\
\text { MUST); integrità cutanea (Braden) }\end{array}$ & & $\begin{array}{l}\text { Rivalutare periodicamente le procedure } \\
\text { di riconciliazione della terapia nel } \\
\text { processo di revisione della qualità }\end{array}$ \\
\hline \multirow[t]{2}{*}{$\begin{array}{l}\text { Educazione/Promozione } \\
\text { dell'autocura }\end{array}$} & $\begin{array}{l}\text { Identificare i bisogni educativi del } \\
\text { paziente e dei caregivers: } \\
\text { riconoscimento precoce dei sintomi } \\
\text { di peggioramento clinico; azioni da } \\
\text { mettere in atto in caso di emergenza } \\
\text { e lista dettagliata di recapiti ed } \\
\text { istruzioni rafforzative; riconoscimento } \\
\text { e monitoraggio dei fattori di rischio; } \\
\text { correzione degli stili di vita; autocura }\end{array}$ & $\begin{array}{l}\text { Avvalersi di metodi per } \\
\text { l'insegnamento agli adulti } \\
\text { evidence-based come: } \\
\text { Teach-Back e Coaching }\end{array}$ & $\begin{array}{l}\text { Partecipare a gruppi di promozione } \\
\text { relativamente alla Health Literacy }\end{array}$ \\
\hline & Predisporre piani educativi strutturati & & \\
\hline \multirow[t]{2}{*}{ Collaborare } & $\begin{array}{l}\text { Presidiare la collaborazione tra operatori } \\
\text { mantenendo una cultura positiva }\end{array}$ & & $\begin{array}{l}\text { Promuovere la formazione di team } \\
\text { multidisciplinari in termini di dimissioni } \\
\text { difficili }\end{array}$ \\
\hline & $\begin{array}{l}\text { Utilizzare quando possibile piattaforme } \\
\text { digitali come mezzo di comunicazione }\end{array}$ & & \\
\hline \multirow[t]{3}{*}{ Promuovere la continuità } & $\begin{array}{l}\text { Redigere relazioni di dimissione } \\
\text { sia per i pazienti/caregivers che per i } \\
\text { clinici riceventi }\end{array}$ & $\begin{array}{l}\text { Presidiare la comunicazione } \\
\text { di un piano comune tra i clinici } \\
\text { inviati e riceventi }\end{array}$ & $\begin{array}{l}\text { Sviluppare protocolli di comunicazione } \\
\text { e procedure per supportare l'accettazione } \\
\text { la dimissione e il follow-up }\end{array}$ \\
\hline & $\begin{array}{l}\text { Preparare il paziente e i caregivers } \\
\text { su cosa li aspetta nel nuovo sito di cura }\end{array}$ & & \\
\hline & $\begin{array}{l}\text { Seguire protocolli di comunicazione } \\
\text { e procedure per supportare l'accettazione, } \\
\text { la dimissione e il follow-up }\end{array}$ & & \\
\hline
\end{tabular}


Tabella 27. Nursing clinical competence: il fine vita.

\begin{tabular}{|c|c|c|}
\hline \multicolumn{3}{|c|}{ ASSISTENZA AL PAZIENTE IN FINE VITA ${ }^{271-279,409-413}$} \\
\hline Professionalità di base & Professionalità ottimale & Professionalità distintiva \\
\hline $\begin{array}{l}\text { Riconoscimento e monitorare segni e sintomi } \\
\text { di terminalità con particolare attenzione } \\
\text { alla gestione del dolore con l'obiettivo di } \\
\text { personalizzare il più possibile le cure e di } \\
\text { conseguenza migliorare la qualità di vita } \\
\text { del paziente }\end{array}$ & $\begin{array}{l}\text { Educare e condividere le informazioni con } \\
\text { gli individui e le loro famiglie per quanto riguarda: } \\
\text { farmaci (vie di somministrazione ed effetti } \\
\text { indesiderati); sintomi potenziali, segni fisici di morte } \\
\text { imminente, pratiche di veglia, strategie di cura di sè }\end{array}$ & $\begin{array}{l}\text { Partecipare a programmi di studio al fine di pianificare } \\
\text { e mettere in atto contenuti specializzati nelle cure di } \\
\text { fine vita }\end{array}$ \\
\hline $\begin{array}{l}\text { Monitorare costantemente eventuali effetti } \\
\text { indesiderati e conoscere le interazioni tra farmaci }\end{array}$ & $\begin{array}{l}\text { Valutano la comprensione della famiglia } \\
\text { su ciò che accade in questa fase della vita }\end{array}$ & $\begin{array}{l}\text { Promuovere la considerazione della morte come } \\
\text { processo naturale }\end{array}$ \\
\hline \multirow[t]{2}{*}{$\begin{array}{l}\text { Pianificare ed effettuare la cura del corpo } \\
\text { dopo la morte }\end{array}$} & $\begin{array}{l}\text { Utilizzano strategie di comunicazione efficace } \\
\text { per facilitare la relazione sul fine vita per } \\
\text { supportare paziente e famiglia dal punto di vista } \\
\text { pratico ed emotivo e nella pianificazione del lutto }\end{array}$ & Conoscere e diffondere le questioni etico-legislative \\
\hline & & $\begin{array}{l}\text { Organizzare momenti di riflessione sull'esperienza } \\
\text { personale e organizzativa per migliorare la pratica } \\
\text { quotidiana promuovendo strategie di coping }\end{array}$ \\
\hline
\end{tabular}




\section{Bibliografia}

1. Massai D, Amerini A, et al. Perché l'analisi delle competenze. La valutazione delle competenze. L'infermiere. 2007; (4):2-40.

2. Saiani L, Dimonte V, et al. Il dibattito sullo sviluppo di competenze specialistiche/avanzate degli infermieri. Assistenza infermieristica e ricerca. 2006; (35):116-21

3. Federazione Nazionale Collegi Infermieri. Evoluzione delle competenze infermieristiche. Documento FNOPI. N.79 del 25 Aprile 2015. Available at: http://www.fnopi .it/archivio_news/pagine/95/15\%20-\%20Comunicato \%20stampa\%204\%20luglio\%202015\%20ALL.pdf

4. Brugnolli A, Campagna S, et al. I bisogni di competenza specialistica percepiti dagli infermieri. Assistenza infermieristica e ricerca. 2006; (35):122-27.

5. Thiella M, Saiani L, et al. L'offerta di formazione post laurea per infermieri nelle Università italiane. Assistenza infermieristica e ricerca. 2006; (35):128-31.

6. Dellai M, Mortari L, et al. Self-assessment of nursing competencies-validation of the Finnish NCS instrument with Italian nurse. Scand J Caring Sci. 2009; 23(4):738-91.

7. Sastre-Fullana P, De Pedro-Gòmez J, et al. Competency frameworks for advanced practice nursing: a literature review. International Council of Nurses. 2014; 534-542

8. Benci L. Le competenze avanzate e la cabina di regia: riflessioni giuridiche. Articolo internet: www.lucabenci. it. 2014. Available at: http://www.lucabenci.it/ wp-content/uploads/2014/01/Competenze-avanzate-sito.pdf

9. Dellai M, Mortari L, et al. Self-assessment of nursing competencies-validation of the Finnish NCS instrument with Italian nurse. Scand J Caring Sci. 2009; 23(4):738-91.

10. Koskinen L, Mikkonen I, et al. Advanced practice nursing for enduring health needs management: a global perspective. Nurse Education Today. 2012;32(5):540-4.

11. Donald F, Martin-Misener R, et al. A systematic review of the effectiveness of advanced practice nurse in longterm care. Journal of advanced nursing. 2013;69(10): 2148-61.

12. Saiani L, Dimonte V, et al. Il dibattito sullo sviluppo di competenze specialistiche/avanzate degli infermieri. Assistenza infermieristica e ricerca. 2006;(35):116-21.

13. Mc Donnell A, Goodwin E, et al. An evaluation of the implementation of Advanced Nurse Practitioner (ANP) roles in an acute hospital setting. Journal of advanced nursing. 2015;(71):789-99.

14. Mathieu G, Greco A, et al. La clinical competence in Medicina interna. Italian Journal of Medicine. 2011;(5): 17-29.

15. Nardi R, Mathieu G et al. Modelli di valutazione della clinical competence del medico specialista internista ospedaliero. Italian Journal of Medicine. 2011; (5S): S33-S45.

16. Halcomb E, Stephens M. et al. Nursing competency standards in primary health care: an integrative review. Journal of Clinical Nursing. 2016; (25):1193-205.

17. World Health Organization. Noncommunicable diseases. Articolo internet. 2017 Available at: http://www. who.int/mediacentre/factsheets/fs355/en/

18. Istituto Superiore di Sanità. Malattie Croniche. Epicen- tro. 2017. Available at: http://www.epicentro.iss.it/temi/ croniche/croniche.asp

19. Ciaccio S, Valentini U. Il ruolo dell'educazione terapeutica nella cronicità. MeDia. 2011; 11:139-144.

20. World Health Organization. Assessing national capacity for the prevention and control of noncommunicable diseases. Articolo internet. 2015. Available at: http:// apps.who.int/iris/bitstream/10665/246223/1/97892415 65363-eng.pdf

21. Agenzia Italiana del Farmaco. Aderenza alle terapie e strategie per migliorare l'uso sicuro ed efficace dei farmaci. Articolo internet. 2014 Available at: http://www. aifa.gov.it/content/aderenza-alle-terapie-e-strategiemigliorare-1\%E2\%80\%99uso-sicuro-ed-efficace-deifarmaci

22. Bodenheimer T, Lorig K, Holman H, Grumbach K. Patient Self-management of Chronic Disease in Primary Care. Journal American Medical Association. 2002; 288(19): 2469-2475.

23. Prosperi E. La malattia cronica. Articolo internet. 2010 Available at: http://www.psicoclinica.it/la-malattiacronica.html

24. Organizzazione Mondiale della Sanità. Educazione terapeutica del paziente. Articolo internet. 1998 Available at: http://www.diabeteitalia.it/download.aspx?sfn=c35 b6b95-4cb1-4ad0-a5af-c0374d433ae5)

25. Gensini GF, Rostagno C. Scompenso cardiaco. Firenze, SEE, 1998.

26. Yancy CW, Jessup M, Bozkurt B, et al. 2017 ACC/AHA/HFSA Focused Update of the 2013 ACCF/AHA Guideline for the Management of Heart Failure: A Report of the American College of Cardiology/American Heart Association Task Force on Clinical Practice Guidelines and the Heart Failure Society of America. J Am Coll Cardiol 2017;Apr 28

27. Ponikowski P, A Voors A, D Anker S et al. 2016 ESC Guidelines for the diagnosis and treatment of acute and chronic heart failure: The Task Force for the diagnosis and treatment of acute and chronic heart failure of the European Society of Cardiology (ESC) Developed with the special contribution of the Heart Failure Association (HFA) of the ESC. European Heart Journal, Volume 37, Issue 27, 14 July 2016, Pages 2129-2200

28. NHS Quality Improvement Scotland (NHS QIS) Clinical Standards Heart Disease. Apr 2010. Available at: http://www.healthcareimprovementscotland.org/programmes/cardiovascular_disease/heart_disease/heart_d isease_standards.aspx

29. NHS Quality Improvement Scotland (NHS QIS) Clinical Standards Heart Disease. Apr 2010. Available at: http://www.healthcareimprovementscotland.org/programmes/cardiovascular_disease/heart_disease/heart_d isease_standards.aspx

30. Cartabellotta A, Iannone $\mathrm{P}$, Linee guida per la diagnosi e il trattamento dello scompenso cardiaco acuto, Evidence, open access journal. Published by the GIMBE Foundation, 2015

31. Walker D, Kemp E. A guide for review and improvement of hospital based heart failure services NHS Improvement Heart. 2013. Available at: https://www.slideshare. net/NHSImprovement/a-guide-for-review-and-improvement-of-hospital-based-heart-failure-services

32. Scottish Intercollegiate Guidelines Network. SIGN 147. Management of chronic heart failure A national 
clinical guideline. March 2016. Available at: https://www.sign.ac.uk/assets/sign1472.pdf

33. Ezekowitz, Justin A. et al. 2017 Comprehensive Update of the CCS Guidelines for the Management of Heart Failure. Can J Cardiol 2017;33:1342.

34. Gensini GF, Fabbri LM, Fini M, NozzoliC. La Medicina della Complessità BPCO e comorbidità. Firenze University Press. 2010.

35. Mathers CD, Loncar D. Chronic obstructive pulmonary disease (COPD). PLoS Medicine. 2006; 209-224.

36. Corsico AG, Levato G, Melani A, Pistelli R, Favaretti C, Kheiraoui F, Curradi G, Santacroce R. Health Technology Assessment di Anoro ${ }^{\circledR}$ nel trattamento della BPCO. Quaderni dell'Italian Journal of Public Health. 2016; Volume 5, Number 2.

37. Agusti A, Decramer M, Celli BR, Chen R, Criner G, Frith P, Halpin D, López Varela MV, Nishimura M, Rodriguez-Roisin R, Vogelmeier C. Strategia globale per la diagnosi, il trattamento e la prevenzione della BPCO (report 2017). GOLD. Progetto Mondiale Broncopneumopatia Cronica Ostruttiva. 2017. Available at: http://www.goldcopd.it/wp-content/uploads/materiali/ 2017/GOLD_workshop_report_2017.pdf

38. Bonini M, Cerullo D, Salvadori R, Torselli R. Percorso broncopneumopatia cronica ostruttiva (BPCO) e insufficienza respiratoria in BPCO EXPANDED CHRONIC CARE MODEL. EpiCentro. Available at: http://www. epicentro.iss.it/igea/raccolta/Allegati/toscana/PROGETTO_CHRONIC_BPCO.pdf)

39. Lopes Pegna A, Rossi M, Corrado A, Augustynen A, Villella G, Bonanni D. Linee guida sull'insufficienza respiratoria. SNLG-Regioni - Linee guida sull'insufficienza respiratoria. 2014. Available at: http://www.snlgiss.it/cms/files/LG toscane_BPCO 2011 def.pdf)

40. Ko FW, Chan KP, Hui DS, Goddard JR, Shaw JG, Reid DW, Yang IA. Acute exacerbation of COPD. Respirology. 2016; 21(7) 1152-65.

41. Rondelli R, Perrella A, Ricceri V, Castagna ML. Sanità d'iniziativa percorso assistenziale per la gestione della broncopneumopatia cronica ostruttiva. EpiCentro. 2010. Available at: http://www.epicentro.iss.it/igea/raccolta/Allegati/toscana/percorso_bpco_ultima_versione_\%20corretta.pdf

42. Istituto Superiore di Sanità. Linee Guida toscane per la gestione della BPCO. Sistema Nazionale Linee Guida. 2011 Available at: http://www.snlg-iss.it/cms/files/ LG_toscane_BPCO_2011_def.pdf

43. Global Initiative for $\bar{C}$ hronic Obstructive Lung Disease. Strategia globale per la diagnosi, il trattamento e la prevenzione della BPCO. Report 2017. Linee Guida. 2017 Available at: http://www.goldcopd.it/wp-content/uploads/materiali/2017/GOLD_workshop_report_2017.pdf

44. Zwerink M, Brusse-Keizer M, van der Valk PD, Zielhuis GA, Monninkhof EM, van der Palen J, Frith PA, Effing T. Self management for patients with chronic obstructive pulmonary disease. Cochrane Database Syst Rev. 2014; (3) CD002990.

45. Chang PE1, Wong GW, Li JW, Lui HF, Chow WC, Tan CK. Epidemiology and Clinical Evolution of Liver Cirrhosis in Singapore. Ann Acad Med Singapore. 2015 Jun;44(6):218-25.

46. Tsochatzis EA, Bosch J, Burroughs AK. Liver cirrosi. Lancet.2014;383(9980):1749-61

47. Fedeli U, Schievano E, Lisiero M et al. Descriptive epi- demiology of chronic liver disease in northeastern Italy: an analysis of multiple causes of death. Popul Health Metr. 2013; 11: 20.

48. Longo, Fauci K. Harrison's Principles of internal Medicine. Cirrhosis and its complications. In: McGraw Hil, ed. Chapter 308. 18th ed.;2013

49. Elliot B. Tapper. Michael Volk. Strategies to reduce 30day readmissions in patients with cirrosi. Curr Gastroenterol Rep.2017.19:1

50. Escobar Gimenes FR, Reis RK, Costa dos Santos da Silva P et al. Nursing Assessment Tool for People With Liver Cirrhosis. Gastroenterol Nurs. 2016 Jul; 39(4): 264-272

51. Orr JG, Homer T, Ternent L, Newton J, McNeil CJ, Hudson M, Jones DE. Healthrelated quality of life in people with advancedchronicliverdisease. J Hepatol. 2014 Nov;61(5):1158-65

52. Ruiz-Margáin A, Macías-Rodríguez SU, Ríos-Torres SL et al...Effect of a high-protein, high-fiber diet plus supplementation with branched-chain amino acids on the nutritional status of patients with cirrhosis. Rev Gastroenterol Mex. 2018 Jan - Mar;83(1):9-15

53. Chopra S. MACP Patient education: Cirrhosis (Beyond the Basics). Articolo internet. 2016. Available at: https://www.uptodate.com/contents/cirrhosis-beyondthe-basics? source $=$ search_result\&search $=$ patient $\%$ 20education:\%20 cirrhosis $\% 20$ (beyond $\% 20$ the $\% 20$ basi c)\&selectedTitle $=4 \sim 150$

54. Neoptolemos JP, Raraty M, Finch M, et al. Acute pancreatitis: the substantial human and financial costs. Gut. 1998; 42:886-91.

55. Lankisch PG, Apte M, Banks PA. Acute pancreatitis. Lancet. 2015 Nov 21;386

56. Smeltzer SC, Bare BG. Infermieristica medico-chirurgica. Casa Editrice Ambrosiana, Milano. 1996. Vol.1

57. Ministero della salute. Dipartimento della programmazione e dell'ordinamento del sistema sanitario nazionale. Direzione Generale per la programmazione sanitaria. Commissione nazionale diabete. Piano sulla malattia diabetica. Articolo Internet. 2017 Available at: http: www.salute.gov.it/imgs/C_17_pubblicazioni 1885 allegato.pdf

58. Chiandetti R, Branca MT, Cucco L, Speese K. Il Diabete in Ospedale: ruolo dell'infermiere. Giornale Italiano di Diabetologia e Metabolismo 2017;37:123-131

59. Gnerre P, Bozzano $\mathrm{C}$ et al. Delirium nell'anziano. Quaderni -Italian Journal of Medicine 2013; 1, 1-12

60. National Institute of Clinical Excellence. Delirium: diagnosis, prevention and management, clinical guideline. CG103. 2010 Available at: https://www.nice. org.uk/guidance $/ \operatorname{cg} 103$

61. SNLG regioni. Delirium: diagnosi, prevenzione e trattamento., Linea Guida 103 del "National Clini-cal Guideline Centre for Acute and Chronic Conditions". Linea Guida. 2011. Available at: http://www. regione.toscana.it/documents/10180/320308/Delirium/ 875530c0-5774-431f-b1f0-59a54e9e50ed)

62. Registered nurses' association of Ontario. Delirium, depression, dementia in older adults: assessments and care. $2^{\circ}$ edition. Best practice guidelines. 2016. Available at: http://rnao.ca/bpg/guidelines/assessment-and-care-older-adults-delirium-dementia-anddepression

63. Neufeld KJ, Due J. et al. Antipsychotic Medication for 
Prevention and Treatment of Delirium in Hospitalized Adults: A Systematic Review and Meta-Analysis. Journal of American Geriatric Society. Apr. 2016; 63(4) 705-14.

64. Carbone M., Gugliucci M.R. Delirium and the family caregiver: the need for evidence-based edu-cation interventions. The Gereontologist 2015; 55 (3) 345-52.

65. American Psychiatric Association. DSM-V (Diagnostic and Statistical Manual of Mental Disorders) 5th Ed. Washington DC, New School Library, 2013: 155

66. dos Santos Damásio Silva D, Vieira da Silva Tavares N et. Al. Depression and suicide risk among Nursing professionals: an integrative review. Rev Esc Enferm USP. 2015 Dec;49(6):1027-36.

67. World Health Organisation. Depression. Articolo internet. 2017 . Available at: http://www.who.int/mediacentre/factsheets/fs369/en/

68. Temel M, Kutlu FY. Gordon's model applied to nursing care of people with depression. Int Nurs Rev. 2015 Dec;62(4):563-72

69. Lill S. Depression in Older Adulta in Primary Care: An Intergrative Approach to Care. J Holist Nurs. University of Pennsylvania, Philadelphia. 2015 Sep;33(3):280-8

70. Bruce ML. Caring for Depression in Older Home Helth Patients. J Psychosoc Nurs Ment Health Serv. 2015 Nov;53(11):25-30

71. Ministero della Salute. Criteri di appropriatezza clinica, tecnologica e strutturale nell'assistenza del paziente complesso. Quaderni del Ministero della salute, n. 23 settembre-ottobre 2013. Available to: www. quadernidellasalute.it

72. American Association of Colleges of Nursing. Scope and standards for acute care nurse practitioner practice. Articolo internet. 2012. Available at: https://www.aacn. org/nursing-excellence/aacn-standards

73. American Association of Colleges of Nursing. AdultGerontology Acute Care And Primary Care NP Competencies. Articolo internet. 2016. Available to: http://www.aacnnursing.org/Portals/42/AcademicNursing/CurriculumGuidelines/Adult-Gero-NP-Comp2016.pdf

74. Department of Health - CNO Directorate - PLT . Advanced Level Nursing: A Position Statement. Articolo internet. 2010. Available to https://www.rcn.org.uk/library/subject-guides/advanced-nursing-practice

75. Campanini M, Fontanella A, Nozzoli C, Mazzone A, Nardi R. La Medicina Interna ospedaliera: tra complessità ed intensità assistenziale verso una medicina sostenibile. Italian journal of medicine. 2014(2)

76. Panigada G, Campanini M, Fontanella A, Nardi R. Aspetti clinico-organizzativi nella degenza medica ospedaliera in Italia: il ruolo della Medicina Interna nel Dipartimento Medico e continuità assistenziale. Italian journal of medicine 2015(3)

77. Mongardi M, Bassi E, Di Ruscio E. Ospedale per Intensità di cura: strumenti per la classificazione dei pazienti. DGSPS, Servizio Presidi Ospedalieri, Regione Emilia-Romagna. Articolo internet. 2015. Available to: http://salute.regione.emilia-romagna.it/documentazione/rapporti/201 cospedale-per-intensita-di-curastrumenti-per-la-classificazione-dei-pazienti201d-febbr aio-2015/view

78. American Association of Colleges of Nursing. Scope and standards for acute and critical care nursing prac- tice. Articolo internet. 2015 Available at: https:// www.aacn.org/nursing-excellence/aacn-standards

79. The National Institute for Health and Care Excellence. Clinical Guideline 50 Acutely ill adults in hospital: recognising and responding to deterioration. Articolo internet. 2007. Available at https://www.nice.org. uk/guidance/cg50

80. Deakin CD, Nolan JP, Soar J, et al. European Resuscitation Council Guidelines for Resuscitation 2010 Section 4. Adult advanced life support. Resuscitation. 2010;81(10):1305-52.

81. Cei M, Bartolomei C, Mumoli N. In-hospital mortality and morbidity of elderly medical patients can be predicted at admission by the Modified Early Warning Score: A prospective study. Int J Clin Pract. 2009;63 (4):591-5.

82. Contursi V, Zaninelli A, Maso, et al. Manuale pratico per la gestione dell'ipertensione arteriosa in Medicina Generale. Italian Journal of Primary Care. Volume 6, Numero 3, novembre 2016.

83. Di Folca A, Pesavento R et al. Modello di Percorso Assistenziale della Trombosi Venosa Profonda. SIAPAV. 2013

84. ISTAT. Mortalità e Cause sul territorio. E-dating 2017. Available at: http://dati.istat.it/ on Jul 2017.

85. Geerts WH, Bergqvist D et al. Prevention of venous thromboembolism: American College of Chest Physicians Evidence-Based Clinical Practice Guidelines (8th Edition). CHEST. 2008 Jun;133(6):381-453

86. Campanini M, Gussoni $\mathrm{G}$ et al. Fattori di rischio per tromboembolismo venoso e profilassi nei pazienti ricoverati in Medicina Interna: analisi dallo studio FADOI "GEMINI". Italian Journal of Medicine. 2010;4: 23-31

87. Istituto Superiore di Sanità. Linee Guida per la Valutazione e la Riduzione del Rischio TEV Correlato. Sistema Nazionale Linee Guida. 2017. Available at: http://www.snlg-iss.it/cms/files/LG_Toscana_ TEV.pdf

88. Hillegass E, Puthoff M et al. Role of Physical Therapists in the Management of Individuals at Risk for or Diagnosed With Venous Thromboembolism: EvidenceBased Clinical Practice Guideline. Physical Therapists. 2016 Feb;96(2):143-66

89. Beck D M. Venous Thromboembolism (VTE) Prophylaxis: Implications For Medical-Surgical Nurses. Medsurg Nursing. 2006 Oct;15(5): 282-87

90. Kahn S R, Lim W et al. Prevention of VTE in Nonsurgical Patient: Antithrombotic Therapy and Prevention of Thrombosis, 9th ed: American College Chest Physicians Evidence-Based Clinical Practice Guidelines. CHEST. 2012 May;141(2):195-226

91. Kvamme A M, Costanzo C. Preventing Progression of Post-Thrombotic Syndrome for Patients Post-Deep Vein Thrombosis. Medsurg Nursing. 2015 Feb;24(1): 27-34.

92. Kearon C, Elie A et al. Antithrombotic therapy for VTE disease: CHEST guideline and Expert Panel report. CHEST. 2016 Feb;149(2):315-52

93. Kahn S R, Comerota A J et al. The post-thrombotic syndrome: evidence-based prevention, diagnosis, and treatment strategies: a scientific statement from the American Heart Association. American Heart Association. 2014 Oct;130:1636-61. 
94. Watson H G, Keeling D M et al. Guideline on aspects of cancer-related venous thrombosis. British Journal of Haematology. 2015 Jun;170:640-48.

95. Treasure T, Carter K et al. Venous thromboembolism in adults admitted to hospital: reducing the risk. Agency for Healthcare Research and Quality. 2015.

96. Rauen C A, Bridges E et al. Evidence-based practice habits: transforming research into bedside practice. Critical Care Nurse. 2009 Apr;29(2):46-61.

97. Bonner L, Johnson J. Deep vein thrombosis: diagnosis and treatment. Nursing Standard 2014 Jan 28;28(21):51-8.

98. Theofanidis D, Gibbon B.. Nursing interventions in stroke care delivery: An evidence-based clinical review. J Vasc Nurs 2016 Dec;34(4):144-151. doi: 10.1016/j. jvn.2016.07.001.

99. ERC e IRC. Advanced life support: manuale del corso 7 edizione originale; edizione italiana linee guida ERC 2015. IRC EDIZIONI. isbn 978-8895517353

100. Smeltzer SC, Bare BG, Hinkle JL, Cheever KH. Brunner, Suddarth. Infermieristica medico-chirurgica. Casa editrice Ambrosiana, 2010.

101. ISO-SPREAD. Ictus cerebrale: linee guida italiane di prevenzione e trattamento Spread (stroke prevention and educational awareness diffusion) viii edizione. Linee guida. 2017. Available at: www.iso-spread.it.

102. Saiani L, Brugnolli A. Trattato di cure infermieristiche. Casa editrice Idelson-Gnocchi, 2011.

103. Mitchell C, Bowen A, Tyson S, Butterfint Z, Conroy P. Interventions for dysarthria due to stroke and other adult-acquired, non-progressive brain injury. Cochrane Database of Systematic Reviews 2017, Issue 1. Art. No.: CD002088. DOI: 10.1002/14651858. CD002088.pub3.

104. Brady MC, Kelly H, Godwin J, Enderby P, Campbell P. Speech and language therapy for aphasia following stroke. Cochrane Database of Systematic Reviews 2016, Issue 6. Art. No.: CD000425. DOI: 10.1002/ 14651858.CD000425.pub4.

105. Olaiya MT, Cadilhac DA, Kim J, Ung D, Nelson MR, Srikanth VK, Bladin CF, Gerraty RP, Fitzgerald SM, Phan T, Frayne J, Thrift AG. Effectiveness of an Intervention to Improve Risk Factor Knowledge in Patients With Stroke: A Randomized Controlled Trial. Stroke. 2017 Apr;48(4):1101-1103. doi: 10.1161/STROKE AHA.116.016229. Epub 2017 Mar 1.

106. Dudka S, Winczewski P, Janczewska K, Kubsik A, Woldańska-Okońska M. [The education influence on effects of rehabilitation in patients after stroke]. Pol Merkur Lekarski. 2016 Nov 25;41(245):225-230.

107. Dreyer P, Angel S, Langhorn L, Pedersen BB, Aadal L. Nursing Roles and Functions in the Acute and Subacute Rehabilitation of Patients With Stroke: Going All In for the Patient. J Neurosci Nurs. 2016 Apr;48(2):108-15. doi: 10.1097/JNN.0000000000000191.

108. Sura L, Madhavan A, Carnaby G, Crary MA. Dysphagia in the elderly: management and nutritional considerations.Clin Interv Aging. 2012;7:287-98. doi: 10.2147/CIA.S23404. Epub 2012 Jul 30.

109. Canham M. Looking into oropharyngeal dysphagia in older adults. Nursing. 2016 Jun;46(6):36- 42. doi: 10.1097/01.NURSE.0000482867.46299.e5.

110. Malagelada JR, Bazzoli F, Boeckxstaens G, et al. World gastroenterology organisation global guidelines: dysphagia global guidelines and cascades update Septem- ber 2014. J Clin Gastroenterol. 2015 May-Jun; 49(5):370-8. doi: 0.1097/MCG.0000000000000307.

111. KY Chan D, Phoon S, Yeoh E. Australian and New Zealand Society for Geriatric Medicine. Position statement - dysphagia and aspiration in older people. Australas J Ageing. 2011 Jun;30(2):98-103. doi: 10.1111 j.1741-6612.2011.00537.x.

112. Schindler A., Borghi E., Ginocchio D.(2008). Disfagia : definizione, fisiopatologia e epidemiologia. Rivista italiana di fisioterapia e riabilitazione respiratoria. 2008:2. pp. 10-15.

113. Baijens LW, Clavé P, Cras P, Ekberg O, Forster A, Kolb GF, Leners JC, Masiero S, Mateos- Nozal J, Ortega O, Smithard DG, Speyer R, Walshe M. European Society for Swallowing Disorders - European Union Geriatric Medicine Society white paper: oropharyngeal dysphagia as a geriatric syndrome. Clin Interv Aging. 2016 Oct 7;11:1403-1428. eCollection 2016.

114. White GN, O'Rourke F, Ong BS et al. Dysphagia: causes, assessment, treatment, and management. Geriatrics. 2008 May;63(5):15-20.

115. Clarke P, Radford K, Coffey M, Stewart M. Speech and swallow rehabilitation in head and neck cancer: United Kingdom National Multidisciplinary Guidelines. J Laryngol Otol. 2016 May;130(S2):S176-S180.

116. SIGN (2010). Scottish Intercollegiate Guideline Network. Management of patients with stroke: identification and management of dysphagia (119). Articolo internet. 2017. Available at http://www.sign.ac.uk/

117. SPreAD Stroke Prevention and educational Awareness Diffusion. Linee guida italiane di prevenzione e trattamento. VII edizione. Articolo internet. 2016

118. Rudakiewicz J. Methods for managing residents with dysphagia. Nurs Older People. 2015 May;27(4):29-33. doi: 10.7748/nop.27.4.29.e694.

119. Kyle G. Managing dysphagia in older people with dementia.Br J Community Nurs. 2011 Jan;16(1):6-10.

120. R. Suhayda, J. C. Walton. Preventing and managing dehydration. MEDSURG Nursing, 2002, 11 (6): 267277.

121. C. L. Pfenning, C. M. Slovis. Sodium disorder in the emergency department: a review of hyponatremia and hypernatremia. Emergency Medicine Practice, 2012, 14 (10): 1-15.

122. Mohan S, Gu S, Parikh A et al. Prevalence of hyponatremia and association with mortality: Results from NHANES Am J Med. 2013 Dec; 126(12): 1127-37.e1.

123. M. Eijgelsheim, E. J. Hoorn. Hypernatremia: balancing is challenging. The Netherlands Journal of Medicine, 2015, 73 (10): 446-447.

124. Viera J.A., Wouk N. Potassium Disorders: Hypokalemia and Hyperkalemia Am Fam Physician. 2015 Sep 15;92(6):487-495.

125. J. Pepin, C. Shields. Advances in diagnosis and management of hypokalemic and hyperkalemic emergencies. Emergency Medicine Practice, 2012, 14 (2): 1-16.

126. Velissaris D, Karamouzos V, Pierrakos C et al. Hypomagnesemia in Critically Ill Sepsis Patients J Clin Med Res. 2015 Dec; 7(12): 911-918.

127. Editorial. Treatment of severe hypophosphataemia. The Lancet, 1981, 2 (8249): 734.

128. Ruuskanen O, Lahti E, et al. Viral Pneumonia. Lancet. 2011 April; 377 (9773).

129. Kabra SK, Lodha R e Pandey RM, Antibiotics for com- 
munity-acquired pneumonia in children. Cochrane Database Syst Rev. 2013 (Available at: https://www. ncbi.nlm.nih.gov/pubmed)

130. ISTAT. Mortalità e Cause sul territorio. E-dating 2012. Available at: http://dati.istat.it/ on May 2017.

131. Giusti $\mathrm{M}$ et al. Le Infezioni in Medicina. 2010; 3:143153

132. NICE (National Institute for Health and Care Execellente). Pneumonia in adults. Linea Guida. 2016 Available at: https://www.nice.org.uk/guidance/qs110

133. NICE. Pneumonia in adults: diagnosis and management. Linea Guida. 2014 Available at: https://www. nice.org. uk/guidance/cg191

134. Hyuck Kim S, Moon Yun J, Bum Chang C et al. Prevalence of upper gastrointestinal bleeding risk factors among the general population and osteoarthritis patients. World J Gastroenterol. 2016 Dec 28; 22(48): 10643-10652.

135. Tielleman T, Bujanda D, Cryer B. Epidemiology and Risk Factors for Upper Gastrointestinal Bleeding Gastrointest Endosc Clin N Am. 2015 Jul;25(3):415-28.

136. BSGEC. Non-variceal upper gastrointestinal hemorrhage:guidelines. Gut 2002; 51: S1-6

137. Norton I. Upper GI bleeding classification. Gastroenterological society of Australia. 2013

138. Azienda sanitaria ospedaliera San Giovanni Battista di Torino. Gestione dei pazienti con emorragia digestiva alta non da varici. SGIA. 2007(3):7

139. ASGE. The role of endoscopy in the patient with lower GI bleeding. Gastrointestinal endoscopy 2014$79(6): 875-878$

140. Azienda sanitaria ospedaliera San Giovanni Battista di Torino. Gestione dei pazienti con emorragia digestiva alta non da varici. SGIA. 2007(3):70

141. Massini R. Medicina interna. McGraw Hill Education 2013:283-285

142. De Franchis R. Diagnosi e trattamento dell'emorragia digestiva alta non varicosa. Giornale italiano di endoscopia digestiva 2016(6):79

143. Bouix J. Conduites à tenir face aux hémorragies digestives. Revue de l'infermiere, 2010 May;(160): 43-44

144. Singer M, Deutschman CS et al.The Third International Consensus Definitions for Sepsis and Septic Shock (Sepsis-3). JAMA. 2016 Feb;315(8):801-10

145. Rhodes A, Evans LE et al.Surviving Sepsis Campaign: International Guidelines for Management of Sepsis and Septic Shock: 2016.Crit Care Med.2017 Mar;45(3): 486-552

146. Novosad SA, Sapiano MR et al.Vital Signs: Epidemiology of Sepsis: Prevalence of Health Care Factors and Opportunities for Prevention.MMWR Morb Mortal Wkly Rep.2016 Aug;65(33):864-9

147. Rosini J.M, Srivastava N.The 2012 guidelines for severe and septic shock: an update for emergency nursing. J Emerg Nurs. 2013 Nov;39(6):652-6

148. Alberto L, Marshall AP et al.Screening for sepsis in general hospitalized patients: a systematic review. J Hosp Infect. 2017 Aug;96(4):305-15

149. Barbara J, Robert M, et al."Practice standards for electrocardiographic monitoring in Hospital setting. An American Association Scientific Statement from the Councils on Cardiovascular Nursing, Clinical Cardiology and Cardiovascular Disease in the Young". Circulation. 2004; (110):2721-46.
150. Sasso L, Baganasco A, et al. " Portfolio delle competenze delle professioni sanitarie". Mc Graw Hill. 2011

151. Vagnarelli F, Marini M, et al. Ventilazione non invasiva: caratteri generali, indicazioni e revisione della letteratura. Gital Cardiol. 2017; 18 496-504.

152. British Thoracic Society Standards of Care Committee. Guidelines for the Ventilatory Management of Acute Hypercapnic Respiratory Failure in Adults. Thorax 2016;71:ii1-ii35.

153. Battistoni I, Marini M, et al. Ventilazione non invasiva e sedazione: evidenze a supporto e consigli pratici. Gital Cardiol. 2017; (18): 513-18.

154. Venkatesaperumal R, D'Souza D'Souza M, et al. Role of a nurse in non-invaive positive pressure ventilation: a conceptual model for clinical practice. International Journal of Nursing Education 2013;(5):119-23.

155. Gruppo NIV SIMEU. Insufficienza respiratoria acuta: dalla CPAP alla NIV. Torino: C.G. Edizioni Medico Scientifiche; 2015.

156. Dizionario della lingua italiana Zanichelli, 2017

157. Montanari P. "L'utilizzo degli ultrasuoni come supporto tecnico-valutativo dell'assistenza infermieristica". Tesi di Laurea. 2015.

158. Afriat Menihan C, Stringer M, Yehuda I. Association of Women's Health, Obstetrics, and Neonatal Nurses. "ultrasound examinations performed by nurses in obstetric, gynecologic, and reproductive medicine setting: Clinical competencies and education guide ( $3 \mathrm{rd}$ ed.)" 2010

159. Menihan CA. "Application of ultrasound imaging to nursing practice". J Obstet Gynecol Neonatal Nurs. 2011 Sep-Oct;

160. Graven T., Wahba A, Hammer AM, Sagen O, Olsen Ø, Skjetne K, Kleinau JO, Dalen H. "Focused ultrasound of the pleural cavities and the pericardium by nurses after cardiac surgery". Scand Cardiovasc J. 2015 Feb;

161. Lin T., Gifford W., Lan Y., Qin X., Liu X., Wang J., Yang B., You T., Chen K. "Diagnostic accuracy of ultrasonography for detecting nasogastric tube (NGT) placement in adults: A systematic review and meta analysis". Int J Nurs Stud. 2017 Jun;

162. Brachelente C., De Simone A, Cammisa P. "Bladder scanner ed ecografo in ausilio al cateterismo vescicale: evidenze e prospettive" Rivista L'Infermiere N²-2015.

163. Romei L., Sabatini A., Biagioni C., Soldati G. "Ecografia infermieristica" Edizioni Medico scientifiche. 2015

164. Morata L.; Ogilvie C.; Yon J., Johnson A. "Decreasing peripherally inserted central catheter use whit ultrasound-guided peripheral intravenous lines" Journal of Nursing Administration. 2017:47

165. Weiner S. Single-operator Ultrasound-guided IV Placement by Emergency Nurses. Abstract. 2011. Available at: www.ClinicalTrials.gov

166. Gundersen GH, Norekval TM, Haug HH, Skjetne K, Kleinau JO, Graven T, Dalen H. "Adding point of care ultrasound to assess volume status in heart failure patients in a nurse-led outpatient clinic. A randomised study". Heart. 2016

167. Dalen H., Gundersen GH., Skjetne K., Haug HH., Kleinau JO, Norekval TM., Graven T. "Feasibility and reliability of pocket-size ultrasound examinations of the pleural cavities and vena cava inferior performed by nurses in an outpatient heart failure clinic". Eur J Cardiovasc Nurs. 2015. 
168. Baumann BM, McCans K, Stahmer SA, Leonard MB, Shults J, Holmes WC "Volumetric bladder ultrasound performed by trained nurses increases catheterization success in pediatric patients". Am J Emerg Med. 2008.

169. Difonzo M., Coppolecchia M., Colagrande G. Gli errori da somministrazione di farmaci: una survey per valutare le percezioni degli infermieri. Evidence 2013;5(9)

170. Smeulers M, Verweij L, Maaskant J, Boer de M, Krediet P, ElsJ.M.NieveenvanDijkum, Vermeule H. Quality Indicators for Safe Medication Preparation and Administration: A Systematic Review. PLOS ONE. 2015. DOI:10.1371/journal.pone.0122695.

171. Ministero della Salute. Raccomandazione sul corretto utilizzo delle soluzioni concentrate di cloruro di potassio - kcl - ed altre soluzioni concentrate contenenti potassio. Raccomandazione Ministeriale. 2008.

172. Ministero della Salute. Raccomandazione per la prevenzione della morte, coma o grave danno derivati da errori in terapia farmacologica. Raccomandazione Ministeriale. 2008.

173. Ministero della Salute. Raccomandazione per la prevenzione della terapia con farmaci "Look-Alike / Sound-Alike. Raccomandazione Ministeriale. 2010.

174. Ministero della Salute. Raccomandazione per la prevenzione degli errori in terapia con farmaci antineoplastici. Raccomandazione Ministeriale. 2012

175. Pohl J.M., Tanner C., Hamilton A., Kaleba O. E, MPH, Fred D. Rachman D. F., White J., Zheng K. Medication safety after implementation of a commercial electronic health record system in five safety-net practices: A mixed methods approach. JAANP. 2013.

176. Fabbri P., Minchella S., Sandri S. Gli errori nella gestione dei farmaci nell'adulto: una revisione della letteratura. EBN. 2007

177. Prakash V., Koczmara C., Savage P, Trip K, Stewart J, McCurdie T., ACafazzo J., Trbovich P. Mitigating errors caused by interruptions during medication verification and administration: interventions in a simulated ambulatory chemotherapy setting. BMJ Qual Saf 2014;23:884-892.

178. Angela Ruth Savage. Educational audit on drug dose calculation learning in a Tanzanian school of nursing. African Health Sciences Vol 15 Issue 2, June 2015.

179. Mattioli R, Luciani A et al. Linee Guida Tumori Nell'Anziano Edizione 2016. Associazione Italiana di Oncologia Medica. 2016; 7-11.

180. Associazione Italiana di Oncologia Medica. Le cure contro il cancro. Associazione Italiana di Oncologia Medica. Articolo Internet. 2017. Available at: http://www.aiom.it/ pazienti/iniziative-eventi-pubblici/immuno-oncologyacademy/le+cure+contro+il+cancro/1,1334,1

181. Associazione Italiana di Oncologia Medica. Chemioterapia. Articolo Internet. 2017. Available at: http://www. aiom.it/pazienti/iniziative-eventi-pubblici/immuno-oncology-academy/le+cure + contro $+\mathrm{il}+$ cancro/chemioterapia/1,1344,0,

182. Petrelli G, Palmi S. Esposizione professionale a chemioterapici antiblastici: rischio per la riproduzione e strategie per la prevenzione. Istituto Superiore di Sanità. $2002 ; 20$.

183. Smith N. Administering Chemotherapy in Nononcology Settings: A case Study. Clinical Journal of Oncology Nursing. 2011 Aug; 15 (4): 433-5.

184. Canadian Association of Nurses in Oncology/Associa- tion Canadienne des Infirmières en Oncologie. National Strategy For Chemotherapy Administration. Standards and competencies for cancer chemotherapy nursing practice. Articolo Internet. 2011 Available at: https://www.cancercare.on.ca/common/pages/UserFile.aspx?fileId=156524)

185. Istituto Nazionale di Statistica. Annuario Statistico Italiano. E-date internet. 2013.

186. Canadian Cancer Society. Getting chemotherapy. Articolo Internet. 2017 Available at: http://www.cancer. ca/en/cancer-information/diagnosis-and-treatment/ chemotherapy-and-other-drug-therapies/chemotherapy/ getting-chemotherapy/?region=on\#ixzz4otbsRK6Q

187. Eastern Cooperative Oncology Group/American College of Radiology Imaging Network Cancer Research Group. ECOG Performance Status. Articolo Internet. 2017 Available at: http://ecog-acrin.org/resources/ecogperformance-status

188. Associazione Italiana di Oncologia Medica. La Carta dei Servizi dell'Oncologia Italiana - Libro Bianco VI Edizione. 2015 Oct; 268-70, 279.

189. Pitt R. Guideline for the Administration of Anti-cancer Treatment. National Health Service Pan Birmingham Cancer Network. 2012 Apr; reviewed 2015 Apr.

190. Leung M, Bland B, et al. Safe Administration of Systemic Cancer Therapy. Part 1: Safety during chemotherapy ordering, transcribing, dispensing, and patient identification. Cancer Care Ontario/Action Cancer Ontario. $2012 \mathrm{Jul}$; reviewed 2017 Jan.

191. Leung M, Bland B, et al. Safe Administration of Systemic Cancer Therapy. Part 2: Administration of Chemotherapy and Management of Preventable Adverse Events. Cancer Care Ontario/Action Cancer Ontario. 2014 Mar; reviewed 2017 Jan.

192. Sheridan-Leos N. A Model of Chemotherapy Education for Novice Oncology Nurses That Supports a Culture of Safety. 2007 Aug; 11 (4): 545-51.

193. Schneieder F, Faithfull S. What Does a Specialist Nurse Mean Across Europe? European Oncology Nursing Society Newsletter Spring. 2011; 28-31.

194. Crannell C. Chemotherapy Administration: Using Simulation Case-Based Scenarios to Assess Chemiotherapy Competency. Oncology Nursing Forum. 2012 Jan; 39 (1): 19-22.

195. Jacobson J O, Polovich M, et al. American Society of Clinical Oncology/Oncology Nuring Society Chemotherapy Administration Safety Standards. Oncology Nursing Forum. 2009 Nov; 36 (6): 651-8.

196. Rudnitzki T, McMahon D. Oral Agents for Cancer: Safety Challenges and Recommendations. Clinical Journal of Oncology Nursing. 2015 Jun; 19 (3): 41-6.

197. Szetela A B, Gibson D. How the New Oral Antineoplastics Affect Nursing Practice. The American Journal of Nursing. 2007 Dec; 107 (12): 40-8.

198. Mislang A, Wildes T, et al. Adherence to oral cancer therapy in older adults:The International Society of Geriatric Oncology (SIOG) taskforce recommendations. Elsevier Cancer Treatment Reviews. 2017; 57: 58-66.

199. Spoelstra S, Sansoucie H. Putting Evidence Into Practice: Evidence-Based Interventions for Oral Agents for Cancer. Clinical Journal of Oncology Nuersing. 2015 Jun; 19 (3): 60-72. 
200. Wilkes G. Intravenous Administration of Antineoplastic Drugs. Journal of Infusion Nursing. 2009 Sep/Oct; 32 (5): 276-85.

201. Krishnasamy M, Kwork-Wei So W, et al. The Nurse's Role in Managing Chemotherapy-Induced Nausea and Vomiting. Cancer Nursing. 2014; 37 (4): E27-35.

202. Cawley M M, Benson L M. Current Trends in Managing Oral Mucositis. Clinical Journal of Oncology Nursing. 2005 Oct; 9 (5): 584-92.

203. Schulmeister L. Extravagation Management: Clinical Update. Seminars in Oncology Nursing. 2011 Feb; 27 (1): 82-90.

204. Cummings R. The role of the Colorectal Nurse Specialist. European Oncology Nursing Society Newsletter Spring. 2008; 24-5.

205. Vogel W H. The Adavnced Practice Nursing Role in a High-Risk Breast Cancer Clinic. Oncology Nursing Forum. 2003; 30 (1): 115-22.

206. Levine JM. 100 Years of bedsores: how much have we learned? Adv Skin Wound Care. 2018 Mar;31(3):139-141

207. Barakat-Johnson M, Lai M, Barnett C et al. Hospitalacquired pressure injuries: Are they accurately reported? A prospective descriptive study in a large tertiary hospital in Australia. J Tissue Viability. 2018 Jul 10. pii: S0965-206X(17)30144-4

208. Istituto Superiore di Sanita. Ulcere da pressione: prevenzione e trattamento, Regione Toscana. Sistema Nazionale Linee Guida. 2008.

209. Moore ZE, Webster J, Saumiriwo R. Wound-care teams for preventing and treating pressure ulcers. Cochrane Database Syst Rev. 2015 Sep 16;(9):CD011011

210. Cartabellotta A, Paghetti A. Linee guida per la prevenzione e trattamento delle lesioni da pressione nelle cure primarie e in ospedale". Evidence. GIMBE foundation: giugno 2014, volume 6, issue 5, e1000080.

211. EPUAP. Linee Guida Europee per le Ulcere da Pressione. Linea Guida. 2016.

212. Moore Z, Webster J. Dressings and topical agents for preventing pressure ulcers. Cochrane Database Syst Rev. 2013 Aug 18;(8):CD009362

213. Moore Z, Johanssen E, van Etten M. A review of PU prevalence and incidence across Scandinavia, Iceland and Ireland (Part I). Journal of Wound Care 2013;22 (7):1-7.

214. Bernabei R, Manes-Gravina E, Mammarella F. Epidemiology of pressure ulcers. Dipartimento di Scienze Gerontologiche, Geriatriche e Fisiatriche. Universita Cattolica del Sacro Cuore, Roma. 2015

215. Scott W. Medications That Increase the Risk of Pressure Ulcer Development. Emma Lloyd edition. 2011.

216. Amlung SR, Miller WR, Bosley LM. The 1999 national pressure ulcer prevalence survey: a benchmarking approach. Adv Skin Wound Care 2001; 14:297-301.

217. Petrella F, Abbritti F, Bachetti F et al. Progetto S.I.U.C., Studio Italiano Ulcere Da Pressione Cutanee. $1^{\circ}$ gennaio 2015-31 dicembre 2016. Italian Journal of Wound Care. 2017:1(1)

218. Schoonhoven L, Defloor T, Grypdonk MHF. Incidence of Pressure Ulcers due to surgery. Journal of Clinical Nursing 2002; 11: 479-487.

219. Bader D, Bouten C, Colin D et al. Pressure Ulcers. Research. 2005;p 35-38.

220. Molly M. Gadd. Braden scale cumulative score versus subscale scores. J WOCN. january /february 2014;41 (1):86-89.
221. Scalise A. Lesioni cutanee croniche. Ed. Edra. 2015.

222. Lamonica P, Re LG, Lusignani M. Efficacia dei dispositivi antidecubito nella prevenzione delle ulcere da pressione. Sinossi di revisioni sistematiche. L'inferimere, 2017; 54:2:e16-e25.

223. Istituto Superiore di Sanità. Prevenzione e trattamento delle ulcere da pressione: guida rapida di riferimento. Aggiornamento NPUAP/EPUAP/PPPIA, seconda edizione 2014.

224. Blanc G, Meier MJ, Stocco JGD et al. Effectiveness of enteral nutritional therapy in the healing process of pressure ulcers: a systematic review. Rev Esc Enferm USP. 2015 Feb;49(1):152-61

225. Regione Friuli Venezia Giulia. Gestione del rischio clinico: lesioni da pressione, prevenzione e trattamento. Linee guida di riferimento. 2014.

226. Grinspun D. Assessment and Management of Pain, Third Edition. Toronto, ON: Registered Nurses'Association of Ontario. 2013, Dec. Available at: http://rnao.ca/bpg/ guidelines/assessment-and-management-pain

227. Brennan F, Carr DB, Cousins M. Pain Management: A Fundamental Human Right. Section Editor(s): Liu, Spencer S. Anesthesia \& Analgesia. 2007 July. 105 (1): 205-221.

228. Berry PH, Katz JA, Chapman CR, Miaskowski C, Covington EC, McLean MJ, Dahl JL. Pain: Current Understanding of Assesment, Management and Treatments. American Pain Society. 2001 (Available at: http://americanpainsociety.org/uploads).

229. Federazione Nazionale Collegi Ipasvi. Codice Deontologico dell'Infermiere. 2009.

230. Legge 15 marzo 2010, n. 38. Disposizioni per garantire l'accesso alle cure palliative e alla terapia del dolore. Gazzetta Ufficiale n. 65 del 19 marzo 2010 Available at :http://www.parlamento.it/parlam/leggi/100381.htm

231. Horgas AL, Yoon SL, Grall M, Boltz M, Capezuti E, Fulmer T, Zwicker D, editor(s). Pain management in older adults. In: Evidence-based geriatric nursing protocols for best practice. National Guideline Clearinghouse. 4th ed. New York (NY): Springer Publishing Company; 2012 (246-67).

232. Ripamonti C, Santini D, Maranzano E, Berti M, Roila F, on behalf of the ESMO Guidelines Working Group. Management of cancer pain: ESMO Clinical Practice Guidelines. Annals of Oncology, 2012. 23 (7): vii139vii154.

233. American Geriatrics Society Panel on the Pharmacological Management of Persistent Pain in Older Person. The management of persistent pain in older persons. J Am Geriatr Soc. 2009; 57: 1331-1346.

234. American Medical Directors Association (AMDA). Pain Management in the long term care setting. National Guideline Clearinghouse. Columbia (MD):; 2012.(Available at: https://www.guideline.gov/summaries/summary)

235. Eaton LH, Meins AR, Mitchell PH, Voss J, Doorenbos AZ. Evidence-based practice beliefs and behaviors of nurses providing cancer pain management: a mixedmethods approach. OncolNurs Forum. 2015 Mar;42(2): 165-73.

236. Song W, Eaton LH, Gordon DB, Hoyle C, Doorenbos AZ. Evaluation of Evidence-based Nursing Pain Management Practice. PainManagNurs. 2015 Aug;16(4): 456-63. 
237. Abdulla A, Adams N, Bone M, Elliott AM, Gaffin J, Jones D, Knaggs R, Martin D, Sampson L, Schofield P; British Geriatric Society. Guidance on the management of pain in older people. British Geriatrics Society. Age and Ageing; 2013 mar.42 (suppl 1).

238. Faigeles B, Howie-Esquivel J, Miaskowski C, StanikHutt J, Thompson C, White C, Wild LR, Puntillo K. Predictors and use of non pharmacologic interventions for procedural pain associated with turning among hospitalized adults. PainManagNurs. 2013 Jun;14(2):85-93.

239. Geneen LJ, Moore RA, Clarke C, Martin D, Colvin LA, Smith BH. Physical activity and exercise for chronic pain in adults: an overview of Cochrane Reviews (Review). Cochrane Database of Systematic Reviews 2017, Issue 4. Art. No.:CD011279.

240. Busse J. The 2017 Canadian Guideline for Opioids for Chronic Non-Cancer Pain. National Pain Center. Ontario, Canada; 2017.

241. Pittiruti M, Scoppettuolo G. Manuale GAVeCeLT dei PICC e dei Midline. Ed.Edra 2016.

242. Kokotis K. Cost containment and infusion services. J Infus Nurs. 2005 May-Jun;28(3 Suppl):22-32;

243. Bishop L, Dougherty L et al. Guidelines on the insertion and management of central venous access devices in adults. Int J Lab Hematol. 2007 Aug;29(4):26178. (Available

at: http://gavecelt.it/nuovo/biblioteca/linee-guida-bcshscelta-impianto-gestione-cvc).

244. Dariushnia SR, Wallace MJ et al. Quality improvement guidelines for central venous access. J Vasc Interv Radiol. $2010 \mathrm{Jul} ; 21(7): 976-81$.

245. O'Grady NP, Alexander M et al. Guidelines for the prevention of intravascular catheter-related infections. Clin Infect Dis. 2011 May;52(9):162-93.(Available at: http://gavecelt.it/nuovo/biblioteca/cdcguidelines2011).

246. Loveday HP, Wilson JA et al. EPIC3 - National evidence-based guidelines for preventing healthcare-associated infections in NHS hospitals in England. J Hosp Infect. 2014 Jan;86 (Suppl 1):1-70.(Available at: http://gavecelt.it/nuovo/biblioteca/linee-guida-epic2014-traduzione-italiana).

247. Romei L, Sabatini A et al. Ecografia infermieristica. Ed medico scientifiche 2009.

248. Gorski L, Hadaway L et al. INS: Infusion Nursing Standard Practice. J Infus Nurs. 2016 Jan/Feb;39(1).(Available at: http://gavecelt.it/nuovo/biblioteca/ins-guidelines2016-0 and http://www.ins1.org/).

249. Atic M, Tulumovic D et al. The use of ultrasound in determining the position of central venous catheter for hemodialysis- flow signal (video and method). J Vasc Access. 2016;17(4):4 (available at: http://www.vascular-access.info/pub/wocova).

250. Bouaziz H, Zetlaoui PJ et al. Guidelines on the use of ultrasound guidance for vascular access. Anaesth Crit Care Pain Med. 2015 Feb;34(1):65-9.

251. Emoli A, Cappuccio S et al. Il protocollo "ISP" (Inserzione Sicura dei PICC): un "bundle" di otto raccomandazioni per minimizzare le complicanze legate all'impianto dei cateteri centrali ad inserimento periferico (PICC). Assist Inferm Ric 2014;33:82-89 (available at: http://gavecelt.it/nuovo/biblioteca/il-protocolloisp-air-2014).

252. Walston J, Hadley EC, Ferrucci L, Guralnik JM,Newman $\mathrm{AB}$, et al. Research agenda for frailty in older adults: toward a better understanding of physiology and etiology: summary from the American Geriatrics Society/National Institute on Aging Research Conference on Frailty in Older Adults. J Am Geriatr. Soc. 2006; 54:991-1001.

253. Bavazzano A, Badiani E, Bandinelli S, Benvenuti F, et al. Frailty in elderly people. Guideline. Regione Toscana; 2015 [visitato il 12 luglio 2017]. Disponibile da: http://www.snlg-iss.it/cms/files/LG_Frailty_elderly_people_2013.pdf

254. Xue QL. The Frailty Syndrome: Definition and Natural History. Clin Geriatr Med. Elsevier Ltd; 2011;27(1):1-15

255. Lallay F, Crome P. Understanding frailty. Postgraduate Medical Journal. 2007 Jan; 83 (975): 16-20

256. Kresevic DM. Reducing fuctional decline in hospitalized older. American Nurse Association vol. 10 n. 5; 2015 May [visitato il 12 luglio 2017]. Disponibile da: https://www.americannursetoday.com/reducing-functional-decline-hospitalized-older/

257. Buckinx F, Rolland Y, Reginster J-Y, Ricour C, Petermans $J$, Bruyère $O$. Burden of frailty in the elderly population: perspectives for a public health challenge. Arch public Heal = Arch belges santé publique. 2015;73(1):19

258. Bakker FC. Evaluating transition towards tailored hospital care for elderly people. Radboud University; 2015 [visitato il 24 luglio 2017]. Disponibile da: http://repository.ubn.ru.nl/handle/2066/134523

259. Liu CJ, Latham NK. Progressive resistance strenght training for improving physical function in older adults. Cochrane Database Syst Rev. 2009 Jul 8;(3)

260. Lavalle T. Complessità e fragilità, modelli di cura sul territorio: l'esperienza della AUSL di Piacenza. G Gerontol; 2009; 57:343-357

261. AIDA, Advancing Integration for a Dignified Ageing. Linee guida sociosanitarie rivolte a persone anziane e disabili in condizioni di fragilità. 2013 Available at: http://www.projectaida.eu/wp-content/themes/thunderbolt/docs/ docaida-1.pdf

262. Di Cara C. La risposta ai bisogni dell'anziano fragile. Realtà assistenziali a gestione infermieristica. Parliamone; 2015 September; 25: 6-8

263. Boltz M, Greenberg SA. Reducing functional decline in older adults during hospitalization: a best practice approach. 2012; 31

264. Ministero della Salute. Piano Nazionale Cronicità. 2016.

265. Sesona ML, Marrazzo L, Sanson G. Studio prospettico sull'efficacia di un progetto di dimissione difficile e precoce dal Pronto Soccorso di soggetti anziani e fragili. Assist Inferm E Ric. 1 luglio 2011;30(3):117-25.

266. Nardi R, Berti F, Greco A, Scanelli G, Leandri P, Reta $\mathrm{M}$, et al. Complexity in hospital internal medicine departments: what are we talking about? Ital J Med. 19 settembre 2013;7(3):142-55.

267. Naylor MD, Shaid EC, Carpenter D, Gass B, Levine C, Li J, et al. Components of Comprehensive and Effective Transitional Care. J Am Geriatr Soc. 1 giugno 2017;65(6):1119-25.

268. Zhu Q-M, Liu J, Hu H-Y, Wang S. Effectiveness of nurse-led early discharge planning programmes for hospital inpatients with chronic disease or rehabilitation needs: a systematic review and meta-analysis. J Clin Nurs. 1 ottobre 2015;24(19-20):2993-3005.

269. Le Berre M, Maimon G, Sourial N, Guériton M, Vedel 
I. Impact of Transitional Care Services for Chronically Ill Older Patients: A Systematic Evidence Review. J Am Geriatr Soc. 1 luglio 2017;65(7):1597-608.

270. Joo JY, Liu MF. Case management effectiveness in reducing hospital use: a systematic review. Int Nurs Rev. 1 giugno 2017;64(2):296-308.

271.FADOI. Ruolo dell'infermiere nel comfort care del paziente a fine vita in medicina interna. Quaderni. Italian Journal of Medicine. 2017 5(4), 93-101

272. Phillips JL. End of life care pathways in acute and hospice care: an integrative review. Journal of pain and symptom management. 2011:41(5), 940-55.

273. Marie Curie Palliative Care Institute . Liverpool Care Pathway for the Dying Version 12. Liverpool: Marie Curie Palliative Care Institute; 2009. Core Documentation

274. Cartabellotta A. Linee guida per l'assistenza agli adulti nel fine vita. Evidence, 8(6), 1-6. 2016

275. Ferris FD. Care in the last days and hours of life. Canadian Hospice Palliative Care Associatione. 2012

276. Cleary, A. Integrating palliative care into primary care for patients with chronic life-limiting conditions. Nurse Pract, 2016:41(3), 42-8.

277. Istituto Superiore di Sanità. Malattie croniche.: aggiornamenti. Articolo Internet 2017 (10 Luglio). Available at: www.epicentro.iss/temi/croniche/aggiornamento.asp.

278. RNAO. End-of-life Care During te last Days and Hours. ClinicalBest Practice Guideline, 2011:9,51.

279. Sorrel JM. Telling life stories. J Psychosoc Nurs Ment Healt Serv, 2009:47(11), 21-25.

280. Registered Nurses Association of Ontario. Establishing therapeutic relationships. Linea Guida. 2006 Available at: http://rnao.ca/sites/rnao-ca/files/Establishing_Therapeutic_Relationships.pdf

281. Gee P, Greenwood D, Paterniti D, Ward D, Miller L. The eHealth enhanced chronic care model: a theory derivation approach. Journal of Medical Internet Research. 2015; 17(4): 11e86.

282. Registered Nurses Association of Ontario. Strategies to Support Self-Management in Chronic Conditions: Collaboration with Clients. 2010 Available at: http://rnao. ca/sites/rnao-ca/files/Strategies_to_Support_Self-Management_in_Chronic_Conditions_-_Collaboration_ with_Clients.pdf)

283. World Health Organization. Assessing national capacity for the prevention and control of noncommunicable diseases. 2010. Available at: http://apps.who.int/iris/bitstream/10665/246223/1/9789241565363-eng.pdf

284. Joosten A.G, DeFuentes-Merillas L, De Weert G.H, Sensky T, Van der Staak C, de Jong C.A.J. Systematic Review of the Effects of Shared Decision-Making on Patient Satisfaction, Treatment Adherence and Health Status. Psychotherapy and psychosomatics. 2008; 77 : 219-226.

285. Massimi A, De Vito C, Brufola I, Corsaro A, Marzuillo C, Migliara G, et al. Are community-based nurse-led self-management support interventions effective in chronic patients? Results of a systematic review and meta-analysis. PLoS ONE. 2017; 12(3): e0173617.

286. Grover A, Joshi A. An overview of chronic disease models: a systematic literature review. Global Journal Health Science. 2014 Oct 29; 7(2): 210-27.

287. Lhussier M, Carr S, Eaton S, Forster N, et al. Care plan- ning for long-term conditions - a concept mapping. Health Expectations. 2013; 18(5): 605-24.

288. Sterne PP, Grossman S, Migliardi JS, Swallow AD, Nurses' Knowledge of Heart Failure: Implications for Decreasing 30-Day Re-Admission Rates, Medsurg Nurs. 2014

289. Delaney C., Apostolidis B, Lachapelle L, Fortinsky R. Heart Lung, Home care nurses' knowledge of evidencebased education topics for management of heart failure. 2011

290. Mahramus T., Penoyer DA, Frewin S., Chamberlain L, Wilson D, Sole ML, Assessment of an educational intervention on nurses' knowledge and retention of heart failure self-care principles and the Teach Back method, Heart Lung. 2014 May-Jun.

291. Radhakrishnan K, Topaz M, Masterson Creber R., Adapting heart failure guidelines for nursing care in home health settings: challenges and solutions, J Cardiovasc Nurs. 2014

292. Goodlin SJ, Trupp R, Bernhardt P, Grady KL, Dracup $\mathrm{K}$, Development and evaluation of the "Advanced Heart Failure Clinical Competence Survey": a tool to assess knowledge of heart failure care and self-assessed competence Patient Educ Couns. 2007

293. Gusdal AK, Josefsson K, Thors Adolfsson E, Martin L., Nurses' attitudes toward family importance in heart failure care, Eur J Cardiovasc Nurs. 2017 Mar

294. Moser DK, Psychosocial factors and their association with clinical outcomes in patients with heart failure: why clinicians do not seem to care,. Eur J Cardiovasc Nurs. 2002

295. Trupp RJ, Diagnosing Sleep Apnea in Patients Hospitalized With Heart Failure: A Role for Advanced Practice Nurses, AACN Adv Crit Care. 2016

296. Artinian NT, Artinian CG, Saunders MM, Identifying and treating depression in patients with heart failure $\mathrm{J}$ Cardiovasc Nurs. 2004

297. Gura MT, Foreman, Cardiac resynchronization therapy for heart failure management, AACN Clin Issues. 2004

298. Moyer-Knox D, Mueller TM, Vuckovic K, Mischke L, Williams RE, Remote titration of carvedilol for heart failure patients by advanced practice nurses J Card Fail. 2004

299. Adiutori DM, Primary pulmonary hypertension: a review for advanced practice nurses Medsurg Nurs. 2000

300. Pugh RN, Murray-Lyon IM, Dawson JL, Pietroni MC, Williams R. Transection of the oesophagus for bleeding oesophageal varices. Br J Surg. 1973;60(8):646-9.

301. Malinchoc M, Kamath PS, Gordon FD, Peine CJ, Rank $\mathrm{J}$, ter Borg PC. A model to predict poor survival in patients undergoing transjugular intrahepatic portosystemic shunts. Hepatology. 2003;31(4):864-71

302. Eric Goldberg, MD Sanjiv Chopra, MD, MACP. Cirrhosis in adults: Overview of complications, general management, and prognosis. Literature review current through: Jul 2017.

303. Tenner S, Baillie J, De Witt J. American College of Gastroenterology Guideline: management of acute pancreatitis. Am J Gastroenterol 2013;108:1400-15.

304. Mann DV, Hershman MJ, Hittinger R, et al. Multicentre audit of death from acute pancreatitis. Br J Surg. 1994; 81:890-3.

305. Russo MW, Wei JT, Thiny MT, et al. Digestive and liver disease statistics, 2004. Gastroenterology. 2004; 126:1448-53. 
306. Fagenholz PJ, Castillo CF, Harris NS, et al. Increasing United States hospital admissions for acute pancreatitis, 1988-2003. Ann Epidemiol. 2007; 17:491-97.

307. Peery AF, Dellon ES, Lund J, et al. Burden of gastrointestinal disease in the United States: 2012 update. Gastroenterology. 2012; 132:1179-87.

308. Pezzilli R, Zerbi A et al. Consensus guidelines on severe acute pancreatitis Italian Association for the Study of the Pancreas (AISP), Dig Liver Dis. 2015 Jul;47(7): $532-43$

309. Working Group IAP/APA evidence-based guidelines for the management of acute pancreatitis Acute Pancreatitis Guidelines Pancreatology. 2013 Jul-Aug;13(4 Suppl 2):e1-15

310. Tenner S, Baillie J, DeWitt J, Vege SS American College of Gastroenterology guideline: management of acute pancreatitis. Am J Gastroenterol. 2013 Sep;108(9):1400-15; 1416.

311. Sarr MG, Banks PA, Bollen TL, Dervenis C, Gooszen HG, Johnson CD, Tsiotos GG, Vege SS The new revised classification of acute pancreatitis 2012.. Surg Clin North Am. 2013 Jun;93(3):549-62

312. Maraví Poma E, Zubia Olascoaga F, et al SEMICYUC 2012. Recommendations for intensive care management of acute pancreatitis. Med Intensiva. 2013 Apr;37(3):163-79

313. Banks PA, Bollen TL, Dervenis C, Gooszen HG, Johnson CD, Sarr MG, Tsiotos GG, Vege SS Classification of acute pancreatitis 2012: revision of the Atlanta classification and definitions by international consensus. Gut. 2013 Jan;62(1):102-11

314. Maraví Poma E, Laplaza Santos C, et al. Clinical pathways in acute pancreatitis: recommendations for early multidisciplinary management. Scientific Committee of the SEMICYUC. Working Group on Infectious Diseases (GTEI-SEMICYUC). Med Intensiva. 2012 JunJul;36(5):351-7

315. Pezzilli R, Zerbi A, Di Carlo V, Bassi C, Delle Fave GF Practical guidelines for acute pancreatitis.; Working Group of the Italian Association for the Study of the Pancreas on Acute Pancreatitis. Pancreatology. 2010;10(5):523-35

316. Pezzilli R, Uomo G, Zerbi A, Gabbrielli A, Frulloni L, De Rai P, Delle Fave G, Di Carlo V Diagnosis and treatment of acute pancreatitis: the position statement of the Italian Association for the study of the pancreas.; Italian Association for the Study of the Pancreas Study Group. Dig Liver Dis. 2008 Oct;40(10):803-8

317. ADM, SID. Standard italiani per la cura del diabete mellito 2009-2010. Articolo Internet. 2011. Available at: http://www.aemmedi.it/files/Linee-guida_Raccomandazioni/2010/2010-2010_linee_guida.pdf

318. ADA. Diabetes Care. Standard of medical care in diabetes -2013. Jan;36 (Supplement 1): S11-S66. Available at: http://care.diabetesjournals.org/content/36/Supplement_1/S11

319. Janice L. Hinkel, Kerry H. Cheever. Brunner Suddarth. Infermieristica medico-chirurgica vol.1 e vol.2. A cura Nebuloni G. Casa Editrice Ambrosiana, 5 Edizione 2017.

320. Bull M. J., Boaz L.et al. Educating family caregivers for older adults about delirium: a systematic review. Worldviews on Evidence- Based Nursing. 2016; 13(3) 232-40
321.8. Martinez F., Tobar C. et al. Preventing delirium: should non- pharmacological, multicomponent intervention be used? A systematic review and meta- analysis of the literature. Age and Aging. 2015; 44 196-204

322. Cabello M, Mellor-Marsá B, Sabariego C, Cieza A, Bickenbach J, Ayuso-Mateos JL. Psychosocial Features of depression: a systematic literature review. J Affect Disord. 2012 Dec 1;141(1):22-33.

323. Harvath TA, McKenzie G. Depression in older adults. In: Boltz M, Capezuti E, Fulmer T, Zwicker D, editor(s). Evidence-based geriatric nursing protocols for best practice. 4th ed. New York (NY): Springer Publishing Company; 2012. p. 135-62.

324. Mijung Park, PhD, Jürgen Unützer, MD, MPH, MA. Geriatric Depression in Primary Care. Edit. Psychiatr Clin North Am. 2011 June ; 34(2): 469-x.

325. Yolonda Pickett, M.D., M.S., Patrick J. Raue, Ph.D., and Martha L. Bruce, Ph.D., M.P.H. Weill Cornell Medical College. Late-Life Depression in Home Healthcare. Edit Aging health. 2012 June ; 8(3): 273-284.

326. NICE (National Institute for Health and Care Excellence). Depression in adults: recognition and management. Clinical guideline. Published: 28 October 2009. nice.org.uk/guidance $/ \operatorname{cg} 90$

327. NICE (National Institute for Health and Care Excellence). Depression in adults. Quality Standard. Published: 29 March 2011. Available at: nice.org.uk/ guidance/qs8

328. David Ekers, Rebecca Murphy, Janine Archer, Catherine Ebenezer, Deborah Kemp, Simon Gilbody. Nurse delivered collaborative care for depression and longterm physical conditions: A systematic review and meta-analysis. Journal of Affective Disorders 149 (2013)14-22

329. Jutta Dreizler, Andrea Koppitz, Sebastian Probst and Romy Mahrer-Imhof. Including nurses in care models for older people with mild to moderate depression: an integrative review. 2013 John Wiley \& Sons Ltd Journal of Clinical Nursing, 23, 911-926.

330. Meredith Flood, PhD, CNS-BC; and Kathleen C. Buckwalter, $\mathrm{PhD}, \mathrm{RN}, \mathrm{FAAN}$. Recommendations for Mental Health Care of Older Adults Part 1-An Overview of Depression and Anxiety. Journal of Gerontological Nursing • Vol. 35, No. 2, 2009.

331. Higgitt A, Anderson D, Guthrie E, Haddad M, Hosker C, Thomas N. NHS Evidence. Depression in adults with a chronich dphysical health problem: Evidence Update March 2012 Edit by NICE (National Institute for Health and Care Excellence).

332. Trangle M, Gursky J, Haight R, Hardwig J, Hinnenkamp T, Kessler D, Mack N, Myszkowski M. Adult depression in primary care. Bloomington (MN): Institute for Clinical Systems Improvement (ICSI); 2016 Mar. 131

333. Sherry A. Greenberg, MSN, APRN,BC, GNP. The Geriatric Depression Scale: Short Form Depression in older adults is underdiagnosed; try this short, simple tool to screen for depression. AJN t October $2007 \mathrm{t} \mathrm{Vol.}$ 107, No. 10.

334. European Federation of Nurses Associations (2015). EFN Competency Framework. Linee Guida EFN per l'applicazione dell'Articolo 31 all'interno di programmi nazionali di formazione infermieristica. Brussels, Belgium. 
335. Smith GB, Prytherch DR, Schmidt P, et al. Hospitalwide physiological surveillance-A new approach to the early identification and management of the sick patient. Resuscitation. 2006;71(1):19-28.

336. Halm EA, Fine MJ, Kapoor WN, Singer DE, Marrie TJ, Siu AL. Instability on hospital discharge and the risk of adverse outcomes in patients with pneumonia. Arch Intern Med [Internet]. 2002;162(11):1278-84. Available from: http://www.ncbi.nlm.nih.gov/ pubmed/12038946

337. Subbe CP. Validation of a modified Early Warning Score in medical admissions. 2001;94(10):521-6. Available from: https://academic.oup.com/qjmed/article-lookup/doi/10.1093/qjmed/94.10.521

338. Kellett J, Wang F, Woodworth S, Huang W. Changes and their prognostic implications in the abbreviated VitalPAC Early Warning Score (ViEWS) after admission to hospital of 18,827 surgical patients. Resuscitation . 2013;84(4):471-6. Available at: http://dx.doi.org/ 10.1016/j.resuscitation.2012.12.002

339. McGinley A, Pearse RM. A national early warning score for acutely ill patients. BMJ, 2012;345(7422): e5310.

340. Rocco MJ, Papetti A. La valutazione della complessità assistenziale infermieristica in Medicina Interna: Applicazione di due metodi. Ital J Med . 2012;6(1):27-32. Available at: http://dx.doi.org/10.1016/j.itjm.2011.11.001

341. Ministero della Salute. L'Ipertensione arteriosa. Articolo internet. 2016. Available at: http:/www.salute.gov. it/portale/salute/p1_5.jsp?id=18\&area=Malattie_cardiovascolari

342. Mancia G, Fegard R, Narkiewicz K, et al. The Task Force for the management of arterial hypertension of the European Society of Hypertension (ESH) and of the European Society of Cardiology (ESC). 2013 ESH/ESC Guidelines for the management of arterial hypertension. European Heart Journal, Volume 34, Issue 28, 21 July 2013, Pages 2159-2219.

343. Dessi-Fulgheri P, Rapelli A. L'ipertensione arteriosa. Caleidoscopio italiano. ISSN 0394 3291.Numero 149 - aprile 2001. Available at: http://medicalsystems.editoria.com

344. Strocchi E. Fisiopatologia della pressione sanguigna. Lezioni di patologia II edizione. Cap. 37 pp. 1169-1201.

345. Battigelli D, Bottoncelli G, D'Ambrosio G, et al. Guida alla Ipertensione. SIMG (Società Italiana Medicina Generale) Manuale pratico per la gestione dei pazienti con ipertensione arteriosa. A cura dell'Area Cardiovascolare della Società Italiana di Medicina Generale. Aprile 2014, pp. 1-85.

346. Mancia G, Laurent S, Agabiti - Rosel E. Aggiornamento delle linee guida europee per il trattamento dell'ipertensione: documento del comitato della Società Europea dell'ipertensione. Linee Guida. Ipertensione e prevenzione cardiovascolare. Gennaio - Marzo 2010; Volume 17 n. 1 .

347. Meetoo D. In too deep: understanding, detecting and managing DVT. Nursing Standard British Journal of Nursing, 2010, Vol 19, No 162010 Jan 17-23;15(18): 47-54.

348. Genesini GF, Carolei A et al., SPREAD viii edizione. Ictus cerebrale: linee guida italiane di prevenzione e trattamento. Raccomandazioni e sintesi. 2017.(available at: http:// www.iso-spread.it/index.php?azione=capitoli\#end)
349. Casaubon LK, Boulanger JM, Blacquiere D, Boucher S, Brown K, Goddard T, Gordon J, Horton M, Lalonde $\mathrm{J}$, LaRivière $\mathrm{C}$, Lavoie $\mathrm{P}$, Leslie $\mathrm{P}$, McNeill J, Menon BK, Moses B, Penn M, Perry J, Snieder E, Tymianski D, Foley N, Smith EE, Gubitz G, Hill MD, Glasser E, Lindsay P; Canadian Stroke Best Practice Recommendations: Hyperacute Stroke Care Guidelines, Update 2015. Heart and Stroke Foundation of Canada Canadian Stroke Best Practices Advisory Committee. Int J Stroke. 2015 Aug;10(6):924-40. doi: 10.1111/ijs. 12551. Epub 2015 Jul 6.

350. Powers WJ, Derdeyn CP, Biller J, Coffey CS, Hoh BL, Jauch EC, Johnston KC, Johnston SC, Khalessi AA, Kidwell CS, Meschia JF, Ovbiagele B, Yavagal DR; American Heart Association Stroke Council. 2015 American Heart Association/American Stroke Association Focused Update of the 2013 Guidelines for the Early Management of Patients With Acute Ischemic Stroke Regarding Endovascular Treatment: A Guideline for Healthcare Professionals From the American Heart Association/ American Stroke Association. Stroke. 2015 Oct;46(10): 3020-35. doi: 10.1161/STR.0000000000000074. Epub 2015 Jun 29.

351. Toni D, Mangiafico S, Agostoni E et al. Intravenous thrombolysis and intra-arterial interventions in acute ischemic stroke: Italian Stroke Organisation (ISO)SPREAD guidelines. 2015 World Stroke Organization Vol 10, October 2015, 1119-1129.

352. Daviet JC, Bonan I, Caire JM, Colle F, Damamme L, Froger J, Leblond C, Leger A, Muller F, Simon O, Thiebaut M, Yelnik A. Therapeutic patient education for stroke survivors: Non- pharmacological management. A literature review. Ann Phys Rehabil Med. 2012 Dec;55(9- 10):641-56. doi: 10.1016/j.rehab.2012.08.011. Epub 2012 Sep 7.

353. Geeganage C, Beavan J, Ellender S, Bath PM. Interventions for dysphagia and nutritional support in acute and subacute stroke. Cochrane Database Syst Rev. 2012 Oct 17;10:CD000323. doi: 10.1002/14651858.CD000323. pub2.

354. Winstein CJ, Stein J, Arena R, Bates B, Cherney LR, Cramer SC, Deruyter F, Eng JJ, Fisher B, Harvey RL, Lang CE, MacKay-Lyons M, Ottenbacher KJ, Pugh S, Reeves MJ, Richards LG, Stiers W, Zorowitz RD; American Heart Association Stroke Council, Council on Cardiovascular and Stroke Nursing, Council on Clinical Cardiology, and Council on Quality of Care and Outcomes Research. Guidelines for Adult Stroke Rehabilitation and Recovery: A Guideline for Healthcare Professionals From the American Heart Association/ American Stroke Association. Stroke. 2016 Jun;47(6) :e98-e169. doi: 10.1161/STR.0000000000000098. Epub 2016 May 4.

355. Gomes F, Hookway C, Weekes CE; Royal College of Physicians Intercollegiate Stroke Working Party. Royal College of Physicians Intercollegiate Stroke Working Party evidence-based guidelines for the nutritional support of patients who have had a stroke. J Hum Nutr Diet. 2014 Apr;27(2):107-21. doi: 10.1111/jhn.12185. Epub 2013 Nov 20

356. . Zhang J, Yu J, Bao Y, Xie Q, Xu Y, Zhang J, Wang P. Constraint-induced aphasia therapy in post- stroke aphasia rehabilitation: A systematic review and meta-analysis of randomized controlled trialsPLoS One. 2017 Aug 
28;12(8):e0183349. doi: 10.1371/journal.pone.0183349. eCollection 2017.

357. El Hachioui H, Visch-Brink EG, de Lau LM, van de Sandt-Koenderman MW, Nouwens F, Koudstaal PJ, Dippel DW. Screening tests for aphasia in patients with stroke: a systematic review. J Neurol. 2017 Feb;264(2):211-220. doi: 10.1007/s00415-016-8170-8. Epub 2016 Jun 3. Review.

358. Allen L, Mehta S, McClure JA, Teasell R. Therapeutic interventions for aphasia initiated more than six months post stroke: a review of the evidence. Top Stroke Rehabil. 2012 Nov- Dec;19(6):523-35. doi: 10.1310/tsr1906523. Review.

359. Thomas LH, Watkins CL, Sutton CJ, Forshaw D, Leathley MJ, French B, Burton CR, Cheater F, Roe B, Britt D, Booth J, McColl E; Identifying continence options after stroke (ICONS): a cluster randomised controlled feasibility trial. ICONS Project Team and the ICONS Patient, Public and Carer Involvement Groups. Trials. 2014 Dec 23;15:509. doi: 10.1186/1745-621515-509.

360. McLaughlin C;Vine L;Chapman L;Deering P.Whittaker S;Beckly J;Fortun P;Murray IA;Hussaini SH;Michell NP;Stableforth B;Thatcher P;Hare NC;Palmer J;Dalton HR; The management of low-risk primary upper gastrointestinal haemorrhage in the community: a 5-year observational study.; European Journal of Gastroenterology \& Hepatology, 2012 Mar; 24(3): 288-293.

361. Stephens JR;Hare NC; Warshow U;Hamad N;Fellows HJ;Pritchard C; Thatcher P;Jackson L;Michell N;Murray IA;Hussaini SH;Dalton HR;Management of minor upper gastrointestinal haemorrhage in the community using the Glasgow Blatchford Score. European Journal of Gastroenterology \& Hepatology, 2009 Dec; 21(12): 1340-1346.

362. Ely EW, Kleinpell RM et al.Advances in the under standing of cilnical manifestations and therapy of severe sepsis: an update for critical care nurses.Am J Crit Care.2003 Mar;12(2):120-33

363. Hird C.Editorial: How nurses can support the Surviving Sepsis Campaign.J Clinic Nurs.2013 Nov;22(2122):e1-2

364. Peach BC.Implications of the new sepsis definition on research and practice.J Crit Care.2017 Apr;38:259-62

365. Institute for Health and Care Excellence. Sepsis: Recognition, Assessment and Early Management.National. Linea Guida. 2016 Available at: https://www.nice.org.uk/ guidance/ng51

366. National Institute for Health and Care Excellence Sepsis. Qualitystandard. Linea Guida. 2017 Available at: https://www.nice.org.uk/guidance/qs161

367. Keenan S, Sinuff T, et al. Clinical practice guidelines for the use of noninvasive positive-pressure ventilation and noninvasive continuous positive airway pressure in the acute care setting. CMAJ 2011; 183(3): E195-E214.

368. Lapostolle, F., Catineau, J., Garrigue, B., Monmarteau, V., Houssaye, T., Vecci, I., Adnet, F. "Prospective evaluation of peripheral venous access difficulty in emergency care". Intensive Care Medicine 2007;

369. Dwyer S., Johnson N., Giambra B. K., Rineair S. "Best Evidence Statements. Use of ultrasound guidance for peripheral intravenous access in the pediatric population”. National Guideline Clearinghouse $2012 \mathrm{dec}$;

370. Rabindranath KS1, Kumar E, Shail R, Vaux EC. "Ul- trasound use for the placement of haemodialysis catheter". Review Cochran Library. 2011 nov;

371. Kuo CC, Wu CY, Feng IJ, Lee WJ.Hu Li Za Zhi.” Efficacy of Ultrasound-Guided Peripheral Intravenous Access: A Systematic Review and Meta-Analysis". 2016 Dec;

372. Saul D., Ajayi S., Schutzman DL., Horrow MM. "Sonography for Complete Evaluation of Neonatal Intensive Care Unit Central Support Devices: A Pilot Study". J Ultrasound Med. 2016 Jul;

373. Strömberg A., Wodlin P., "Pocket-size ultrasound. A new tool for heart-failure in daily clinical practice?". Editorial, European Journal of Cadiovascular Nursing 2015 Vol.14 (4) 284-285;

374. Baumann BM, McCans K, Stahmer SA, Leonard MB, Shults J, Holmes WC "Volumetric bladder ultrasound performed by trained nurses increases catheterization success in pediatric patients". Am J Emerg Med. 2008 Jan;

375. 1Baumann BM, McCans K, Stahmer SA, Leonard MB, Shults J, Holmes WC."Caregiver and health care provider satisfaction with volumetric bladder ultrasound". Acad Emerg Med. 2007 Oct;

376. Practice Committee of the American Society for Reproductive Medicine. "Position statement on nurses performing limited ultrasound in a gyn/infertility setting". Fertil Steril. 2004 Sep;

377. Gustafsson M, Alehagen U, Johansson P. "Pocket-size ultra- sound examination of fluid imbalance in patients with heart failure: A pilot study of heart failure nurses without prior experience of ultrasonography". Eur J Cardiovasc Nurs 2015;

378. Dalen H, Gundersen GH, Skjetne K, Haug HH, Kleinau JO, Norekval TM, Graven T. "Feasibility and reliability of pocket-size ultrasound examination of the pleural cavities and vena cava inferior performed by nurses in an outpatient heart failure clinic". Eur J Cardiovasc Nurs 2015;

379. Stengel D., Hoenning A., Leisterer J. "Point-of-care ultrasonography for diagnosing thoracoabdominal injuries in patients with blunt trauma”. 2017 May The Cochrane Library;

380. Pittiruti M., Scoppettuolo G. "Manuale GAVeCeLT dei Picc e dei Midline. Indicazione, impianto, gestione".

381. Belo MP, Silva RA, Nogueira IL, Mizoguti DP, Ventura CM. 'Neonatology nurses' knowledge about Peripherally Inserted Central Venous Catheter" Revista Brasileira de Enfermagem, vol.65 no.1 Brasília Jan./Feb. 2012

382. Servizio Sanitario Nazionale Regione Piemonte Asl TO2. Raccomandazioni per l'utilizzo e la gestione degli accessi venosi, centrali e periferici". Overview della letteratura secondaria. 2015.

383. AO Ordine Mauriziano Torino Ospedale Umberto I . L'applicazione delle evidenze scientifiche nell' inserzione e gestione degli accessi venosi. Procedura Generale.., 2014.

384. da Costa Machado Duarte S, Andrade Conceição Stipp M, da Silva MM et al. Adverse events and safety in nursing care. Rev Bras Enferm. 2015 janfev;68(1):136-46

385. American Association of Colleges of Nursing. Scope and standards for acute and critical care nursing practice. Linea Guida. 2015. Available at: https://www.aacn.org /nursing-excellence/aacn-standards

386. American Association of Colleges of Nursing. Scope and 
Standards for Acute Care Clinical Nurse Specialist Practice. Linea Guida. 2010. Available at: https://www.aacn. org/nursing-excellence/aacn-standards

387. Sulosaari V, Suhonen R, Leino-Kilpi H. An integrative review of the literature on registered nurses' medication competence. J Clin Nurs. 2011 Feb;20 (3-4):464-78. doi: 10.1111/j.1365-2702.2010.03228.x. Epub 2010 Aug 24.

388. Landsperger JS, Wheeler AP. Central venous catheter insertion by advanced practice nurses: another demonstrated competency. Crit Care Med. 2014 Mar;42(3): 731-732.

389. Hertzog DR, Waybill PN. Complications and controversies associated with peripherally inserted central catheters. J Infus Nurs. 2008 May-Jun;31(3):159-63.

390. Hentrich M, Schalk E et al. Central venous catheter-related infections in hematology and oncology: 2012 updated guidelines on diagnosis, management and prevention by the Infection Diseases Working Party of the German Society of Hematology and Medical Oncology. Ann Oncol. 2014 May;25(5):936-47.

391. Mermel LA, Allon M et al. Clinical practice guidelines for the diagnosis and management of intravascular catheter-related infection: 2009 Update by the Infectious Diseases Society of America. Clin Infect Dis. 2009 Jul 1;49(1):1-45.

392. Marschall J, Mermel LA et al. Strategies to prevent Central Line-Associated Bloodstream Infection in acute care hospitals: 2014 Update. Infect Control Hosp Epidemiol. 2014 Jul;35(7):753-71.

393. Miller MD, O'Grady NP. Guidelines for the prevention of intravascular catheter-related infections: recommendations relevant to interventional radiology for venous catheter placement and maintenance. J Vasc Interv Radiol. 2012 Aug;23(8):997-1007.

394. Penne K. Using evidence in central catheter care. Semin Oncol Nurs. 2002 Feb;18(1):66-70.

395. Barnes S, Olmsted RN et al. Guide to preventing central line-associated bloodstream infections. APIC 2015. Available at: http://gavecelt.it/nuovo/biblioteca/lineeguida-apic-2015-1

396. Debourdeau P, Kassab Chahmi D et al. 2008 SOR Guidelines for the prevention and treatment of thrombosis associated with central venous catheters in patients with cancer: report from the working group. Ann Oncol. 2009 Sep;20(9):1459-71. Available at: http://gavecelt.it/nuovo/biblioteca/linee-guida-sor

397. Guyatt GH, Akl EA et al. Executive summary: antithrombotic therapy and prevention of thrombosis. 9th edition: American College of Chest Physicians evidencebased clinical practice guidelines. Chest. 2012 Feb;141(2 Suppl):7S-47S. Available at: http://gavecelt.it/nuovo/biblioteca/accp-guidelines-chest-2012

398. Debourdeau P, Farge D et al. International clinical practice guidelines for the treatment and prophylais of thrombosis associated with central venous catheters in patients with cancer. J Thromb Haemost. 2013 Jan;11(1):71-80. Available at: http://gavecelt.it/nuovo/biblioteca/debourdeau-2012-guidelines

399. Andris DA, Krzywda EA. Central venous catheter oc- clusion: successful management strategies. Medsurg Nurs. 1999 Aug;8(4):229-36.

400. Hill J, Broadhurst D, Miller K et al. Occlusion management guideline for central venous access devices. J Vasc Acc. 2013; 7(1). Available at: http://gavecelt.it/nuovo/biblioteca/cvaa-guidelines-vad-occlusion-2013

401. Moureau N, Lamperti M et al. Evidence-based consensus on the insertion of central venous access devices: definition of minimal requirements for training. Br J Anaesth. 2013 Mar;110(3):347-56.

402. Hirschman KB, Shaid E, McCauley K, Pauly MV, Naylor MD. Continuity of Care: The Transitional Care Model. Online J Issues Nurs. 30 settembre 2015;20(3):1.

403. Mistiaen P, Duijnhouwer E, Prins $\square$ Hoekstra A, Ros W, Blaylock A. Predictive validity of the BRASS index in screening patients with post-discharge problems. J Adv Nurs. 1 novembre 1999;30(5):1050-6.

404. Cooke L, Gemmill R, Grant M. Advanced practice nurses core competencies: a framework for developing and testing an advanced practice nurse discharge intervention. Clin Nurse Spec CNS. ottobre 2008;22 (5):218-25.

405. National Guideline Clearinghouse (NGC). Transitional care. In: Evidence-based geriatric nursing protocols for best practice. [Internet]. 2012. Available at: https://guideline.gov/summaries/summary/43940/transitional-care-in-evidencebased-geriatric-nursing-protocols-for-best-practice?q=discharge+planning

406. Shadmi E, Admi H, Ungar L, Naveh N, Muller E, Kaffman M, et al. Cancer care at the hospital-community interface: Perspectives of patients from different cultural and ethnic groups. Patient Educ Couns. aprile 2010;79(1):106-11.

407. National Guideline Clearinghouse (NGC). Transition between inpatient hospital settings and community or care home settings for adults with social care needs. [Internet]. 2015.

408. https://guideline.gov/summaries/summary/49954/transition-between-inpatient-hospital-settings-and-community-or-care-home-settings-for-adults-with-social-careneeds?q=hospital+discharge

409. Lacour, F.(2011, Sep). Palliative care health networks, bridges between hospitaland home Soins(758), 38-39.

410. Vacas Guerrero, M.(2014, Jan-Feb). Adding valueto the care at the final stage of chronic disease. Enfermeria Clinica, 24(1), 74-8. Consultato il giorno 25.09.2017

411. RNAO. End-of-life Care During te last Days and Hours. ClinicalBest Practice Guideline, 9,51. Linea Guida. 2011

412. NICE. Caring for an adult at the end of life-Clinical pathway. 4,5. Linea Guida. 2017

413. RNAO. End-of-life Care During te last Days and Hours. ClinicalBest Practice Guideline, 9,51. Linea Guida. 2011

414. Filannino C, Stornello I, et al. Le politiche di people strategy in sanità: lo sviluppo delle clinical competence per la crescita professionale del dirigente medico. Italian Journal of Medicine. 2011 Mar; 5 (2): S2-16. 


\section{LINEE GUIDA PER GLI AUTORI}

I Quaderni dell 'Italian Journal of Medicine (Quaderni ITJM), costituiscono una collana supplementare solo online annessa alla rivista Italian Journal of Medicine contenente lavori solo in lingua italiana.

I Quaderni ITJM pubblicano:

- Monografie ad hoc individuate dal Presidente FADOI, dal Consiglio Direttivo, dal Board Scientifico o dall'Editor in Chief dell'Italian Journal of Medicine, in funzione del contesto scientifico-istituzionale attuale.

- Monografie ad hoc su temi di particolare rilevanza scientifica a cura della Commissione FADOI Giovani.

- Traduzioni in italiano di alcuni lavori pubblicati sui numeri standard dell'Italian Journal of Medicine, di particolare interesse per la comunità scientifica.

\section{STESURA DEI LAVORI}

I lavori dovranno essere redatti in modo conforme alle linee guida sotto riportate:

- I manoscritti devono essere scritti interamente in lingua italiana, su documento di Word, con carattere Times New Roman/Arial, dimensione 12, formato A4, interlinea doppia e margini $2,54 \mathrm{~cm}$

Parole totali: $\max$ 4000; Sommario/Abstract: $\max 250$ parole; Bibliografia: min 40 voci; Tabelle e Figure: $3 / 5$ totali (le tabelle non devono superare n. 1 pagina del documento in Word).

- La strutturazione del contenuto deve attenersi agli standard internazionali per la Rassegna (Review): i) Abstract riassuntivo dell'intero lavoro; ii) Introduzione al tema trattato; iii) Criteri e strumenti di ricerca (criteri di inclusione/esclusione, banche dati consultate, ...); iv) i successivi paragrafi devono illustrare le più recenti scoperte scientifiche nel settore; v) Conclusioni; vi) Bibliografia.

- La prima pagina deve riportare: i) titolo (in stampatello minuscolo), senza acronimi; ii) nome e cognome per esteso di ciascun autore; iii) affiliazione(i) di ciascun autore, numerate con numeri arabi iv) eventuali ringraziamenti; v) nome e indirizzo postale completi dell'autore corrispondente, corredati da telefono, fax, e-mail; vi) da 3 a 5 parole chiave, separate da virgola. La seconda pagina può riportare: i) contributi degli autori, e.g. informazioni relative a contributi sostanziali delle persone coinvolte nello studio (http://www.icmje.org/\#author); ii) dichiarazione relativa a potenziali conflitti d'interesse; iii) ulteriori informazioni (e.g. fondi, esposizioni durante conferenze...).

- In caso di utilizzo di tabelle, queste devono essere tutte numerate con numeri arabi e citate nel testo in ordine consecutivo (e.g. NON nominare le tabelle come Tabella $1 \mathrm{~A}, 1 \mathrm{~B}, \ldots$ o $1.0,1.1, \ldots$ ). Le tabelle devono essere presentate in formato editabile. Ciascuna tabella deve essere corredata da una breve didascalia; in caso di abbreviazioni, riportare una nota a piè di CIASCUNA tabella che spieghi TUTTE le abbreviazioni presenti in ognuna.

- In caso di utilizzo di figure, queste devono essere inviate in formato tiff o .jpg, allegate al manoscritto in singoli files, secondo le seguenti specifiche:

i) a colori (salvate in modalità CMYK): minimo 300 dpi di risoluzione;

ii) in bianco e nero: minimo $600 \mathrm{dpi}$ di risoluzione;

iii) minimo $17,5 \mathrm{~cm}$ di larghezza.

Ciascuna figura deve essere corredata da una breve didascalia.

$N B$ : In caso di Tabelle/Figure riprese e/o modificate da altri lavori già pubblicati, sarà cura degli autori accertarsi se tali materiali siano o meno coperti da copyright e procurarsi i permessi necessari per la riproduzione. Tali permessi dovranno essere allegati alla versione definitiva del lavoro. L'ufficio editoriale si riserva la facoltà di rimuovere Tabelle/Figure coperte da copyright, se sprovviste dei necessari permessi.

- In caso di utilizzo di abbreviazioni, la prima volta che esse sono citate è necessario scrivere per esteso la definizione+abbreviazione tra parentesi tonde [e.g. risonanza magnetica (RMN)], a seguire si dovrà riportare solo l'abbreviazione (unica eccezione: nei titoli e nelle didascalie di tabelle e figure NON si utilizzano abbreviazioni).

\section{BIBLIOGRAFIA}

Le voci bibliografiche devono essere formattate secondo lo stile Vancouver.

Nella sezione Bibliografia, le voci bibliografiche devono essere numerate consecutivamente nell'ordine in cui appaiono per la prima volta nel testo (NON in ordine alfabetico) e, nel testo, devono essere indicate con numeri arabi in apice. Voci bibliografiche riferite a comunicazioni personali o dati non pubblicati devono essere incorporate nel testo e NON inserite tra le voci numerate [ e.g. Wright 2011, dati non pubblicati) o (Wright 2011, comunicazione personale)]. Le voci bibliografiche nella sezione Bibliografia devono tassativamente essere preparate come segue:

i) più di 3 autori, citare 3 autori, et al. Se il lavoro contiene solo 4 autori, citarli tutti e 4 ;

ii) titolo del lavoro in stampatello minuscolo;

iii) nome della rivista, senza punti, abbreviato secondo gli standard internazionali; in caso di dubbi sulla corretta abbreviazione, fare riferimento ai seguenti siti:

a. ISI Journal Abbreviations Index (http://library.caltech.edu/ reference/abbreviations/);

b. Biological Journals and Abbreviations (http://home.ncifcrf. gov/research/bja/);

c. Medline List of Journal Titles (ftp://ftp.ncbi.nih.gov/pubmed/J_Medline.txt);

iv) inserire l'anno di pubblicazione subito dopo il nome della rivista, seguito da punto e virgola;

v) NON inserire giorno o mese di pubblicazione;

vi) citare solo il volume, seguito dai due punti (NON citare il fascicolo tra parentesi);

vii) abbreviare le pagine, e.g. 351-8

Per accertarsi di aver correttamente formattato le voci bibliografiche, confrontarle con le citazioni in PubMed (http://www.ncbi. nlm.nih.gov/pubmed).

Esempi (prestare attenzione anche alla punteggiatura):

Articolo standard su Rivista

Halpern SD, Ubel PA, Caplan AL. Solid-organ transplantation in HIV-infected patients. N Engl J Med 2002;347:284-7.

Proceedings

Christensen S, Oppacher F. An analysis of Koza's computational effort statistic for genetic programming. In: Foster JA, Lutton E, Miller J, Ryan C, Tettamanzi AG, eds. Genetic programming. EuroGP 2002: Proceedings of the 5th European Conference on Genetic Programming, 2002 Apr 3-5, Kinsdale, Ireland. Berlin: Springer; 2002. pp 182-91.

Articoli i cui autori sono Organizzazioni

Diabetes Prevention Program Research Group. Hypertension, insulin, and proinsulin in participants with impaired glucose tolerance. Hypertension 2002;40:679-86.

Libri

Murray PR, Rosenthal KS, Kobayashi GS, Pfaller MA. Medical microbiology. 4th ed. St. Louis, MO: Mosby; 2002.(CITAZIONE DEL LIBRO INTERO)

Meltzer PS, Kallioniemi A, Trent JM. Chromosome alterations in human solid tumors. In: Vogelstein B, Kinzler KW, eds. The genetic basis of human cancer. New York, NY: McGraw-Hill; 2002. pp 93113.(CITAZIONE DI UN CAPITOLO)

\section{MODALITÀ D'INVIO DEI LAVORI \\ Monografie}

Gli Autori dovranno fare riferimento all'Editor-in-Chief o alle persone da lei/lui designate nelle lettere di invito a scrivere gli articoli programmati.

Per gli articoli inviati da giovani internisti, gli Autori dovranno fare riferimento alla Dr.ssa Paola Gnerre (e-mail: pgnerre@yahoo.it) e agli eventuali altri referenti da lei designati, nelle prime fasi di stesura dei manoscritti, revisioni e correzioni. 


\title{
QUADERNI
}

\author{
dell'Italian Journal
}

of Medicine

La Dr.ssa Gnerre raccoglierà poi le versioni definitive dei lavori di ciascuna monografia e provvederà all'invio di tutti i materiali all'ufficio editoriale.

I lavori solo nella loro versione definitiva e approvata dalla Commissione FADOI Giovani dovranno pervenire all'ufficio editoriale già pronti per l'impaginazione e immediata pubblicazione (già corredati da eventuali permessi per la riproduzione di tabelle e immagini).

\section{Traduzioni}

Previo invito dell'Editor-in-Chief, gli Autori dovranno far pervenire all'ufficio editoriale la versione tradotta in italiano, al seguente indirizzo e-mail: paola.granata@pagepress.org

Il file in formato Word dovrà essere formattato secondo gli standard editoriali della rivista ufficiale ed essere già pronto per impagina- zione e immediata pubblicazione (corredato da eventuali permessi per la riproduzione di tabelle e immagini).

Si prega di inviare le eventuali tabelle in formato editabile e le figure in alta definizione secondo gli standard sopra riportati.

\section{NOTA PER GLI AUTORI}

I lavori pubblicati sui Quaderni ITJM non verranno indicizzati, ma saranno liberamente disponibili in un'apposita sezione del sito FADOI (http://www.fadoi.org/).

Gli Autori i cui lavori siano accettati per la pubblicazione sui Quaderni ITJM e che fossero interessati a vederli pubblicati anche sulla rivista ufficiale, dovranno sottomettere attraverso il sito dell'ITJM (www.italjmed.org) la versione (già tradotta) in inglese e redatta in modo conforme alle linee guida della rivista; seguiranno poi la procedura di selezione tramite peer review e, se accettati, saranno inseriti nel piano editoriale standard.

\section{STAFF EDITORIALE}

Paola Granata, Journal Manager

paola.granata@pagepress.org

Claudia Castellano, Production Editor

Tiziano Taccini, Technical Support
PUBBLICATO DA

PAGEPress Publications

via A. Cavagna Sangiuliani 5

27100 Pavia, Italy

T. +39.0382 .464340$

F: +39.0382 .34872$

\section{agepress}

www.pagepress.org info@pagepress.org

\section{QUADERNI - ITALIAN JOURNAL OF MEDICINE}

Tutti gli articoli pubblicati sui QUADERNI - Italian Journal of Medicine sono redatti sotto la responsabilità degli Autori. La pubblicazione o la ristampa degli articoli della rivista deve essere autorizzata per iscritto dall'editore. Ai sensi dell'art. 13 del D.Lgs 196/03, i dati di tutti i lettori saranno trattati sia manualmente, sia con strumenti informatici e saranno utilizzati per l'invio di questa e di altre pubblicazioni e di materiale informativo e promozionale. Le modalità di trattamento saranno conformi a quanto previsto dall'art. 11 del D.Lgs 196/03. I dati potranno essere comunicati a soggetti con i quali PAGEPress intrattiene rapporti contrattuali necessari per l'invio delle copie della rivista. Il titolare del trattamento dei dati è PAGEPress Srl, via A. Cavagna Sangiuliani 5 - 27100 Pavia, al quale il lettore si potrà rivolgere per chiedere l'aggiornamento, l'integrazione, la cancellazione e ogni altra operazione di cui all'art. 7 del D.Lgs 196/03.

Editore: PAGEPress srl, via A. Cavagna Sangiuliani 5, 27100 Pavia, Italy www.pagepress.org 